\title{
INVESTIGATION OF ENDOSOMAL RECYCLING OF SYNAPTIC VESICLES
}

\author{
Dissertation \\ in partial fulfillment of the requirements for the degree \\ “Doctor of Natural Sciences (Dr. rer. nat.)” \\ in the Molecular Biology Program at the \\ Georg August University Göttingen, Faculty of Biology
}

submitted by

Peer Hoopmann

born in

Thedinghausen, Germany

September 2010 

I hereby declare that I prepared the dissertation entitled "Investigation of Endosomal Recycling of Synaptic Vesicles" on my own and with no other sources and aids than quoted.

Peer Hoopmann 



\section{LIST OF PUBLICATIONS}

Hoopmann P, Punge A, Barysch SV, Westphal V, Bückers J, Opazo F, Bethani I, Lauterbach MA, Hell SW, Rizzoli SO. Endosomal sorting of readily releasable synaptic vesicles. Proc Natl Acad Sci U S A. in press

Hoopmann P, Rizzoli SO, Betz WJ. Imaging synaptic vesicle dynamics with FM dyes. Optical Imaging in Neuroscience (Book chapter). in press

Opazo F, Punge A, Bückers J, Hoopmann P, Kastrup L, Hell SW, Rizzoli SO (2010) Limited Intermixing of Synaptic Vesicle Components upon Vesicle Recycling. Traffic 11:800-812.

Geumann U, Barysch SV, Hoopmann P, Jahn R, Rizzoli SO (2008) SNARE function is not involved in early endosome docking. Mol Biol Cell 19:5327-5337. 


\section{TABLE OF CONTENTS}

List of Publications

Table of Contents II

Acknowledgements IV

Abstract V

List of Figures VI

List of Tables VII

List of Abbreviations VIII

1. Introduction 10

1.1 Pre-Synaptic Function ___ 10

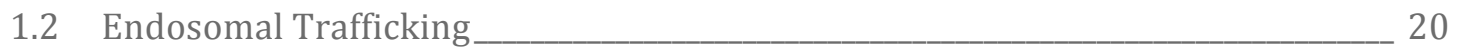

1.3 Endosomes and Synaptic Vesicles ____________ 26

1.4 Aim of the Project ___ 31

2. Materials and Methods ___ 32

2.1 Antibodies __ 32

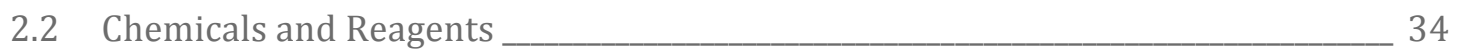

2.3 Microscopy _ـ_ 35

2.4 Neuronal Cell Culture ___ 38

2.5 Preparation of Lumenally Labeled Synaptic Vesicles ___ 38

2.6 PHluorin Expression and Imaging _____ 40

2.7 Photo-oxidation Electron Microscopy _____ 43

2.8 Quantum Dot Imaging and Analysis ______________ 45

2.9 Rab5-GFP Expression and Imaging ___ 46

2.10 Blocking Endosome Function with Soluble Syntaxin 13 Fragment___ 49

2.11 Synaptic Vesicle Marker Surface Immunostaining ___ 51

2.12 Isolated Synaptic Vesicle Immunoassay _____ 52

2.13 Extraction of Cholesterol from Endosomes____ 54

3. Results___ 55

3.1 Endosomal SNAREs Participate in Synaptic Vesicle Recycling ___ 55

3.2 Morphological Evidence for Endosomal Fusion of RRP Vesicles ___ 59 
3.3 Endocytosed Vesicle Protein Reaching Bona Fide Endosomes 65

3.4 Effects of Inhibiting Endosomal Function _ 70

3.5 The Recycling-Step Necessitating Endosomal Sorting ___ 75

3.6 How Can Endosomes Sort Synaptic Vesicle Components?___ 83

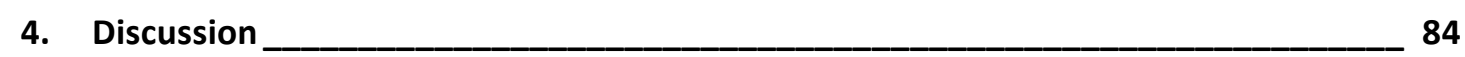

4.1 Exchange of Molecules during Vesicle Recycling ___ 84

4.2 Mechanisms of Endosomal Sorting of Synaptic Vesicles____ 87

4.3 Synaptic Vesicle Pools and Endosomal Recycling ___ 89

4.4 Perspectives for Synaptic Vesicle Recycling __ 90

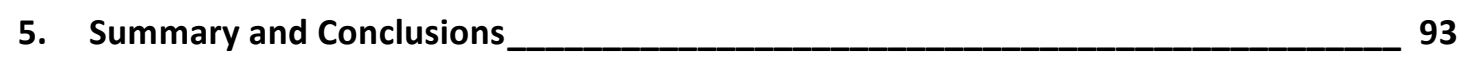

5.1 Do Endosomal Proteins Cycle Between Internal Organelles and the Plasma Membrane upon Stimulation?___ 94

5.2 Do Synaptic Vesicles Fuse with Endosomes During Recycling? ___ 95

5.3 Does Inhibition of Endosomal Function Have an Effect on Any of the Synaptic Vesicle Pools? 95

5.4 Do Vesicle and Plasma Membrane Components Intermix during Synaptic Vesicle Recycling? _ 96

5.5 How Could Endosomal Sorting Maintain Synaptic Vesicle Identity?___ 96

5.6 Open Questions___ 97

Bibliography____ 99

A. Appendix _ـ 109

1. Matlab Routines for pHluorin Analysis __ 109

2. Matlab Routines for in vitro Immunoassay Analysis____ 116 


\section{ACKNOWLEDGEMENTS}

First and foremost I want to thank my supervisor Dr. Silvio Rizzoli for not merely being a supervisor - you are a true mentor. Thank you for teaching me how to do "smart science" and how to be critical. Thank you for your endless support, patience and advice. Thank you for the time you spared for all those instances of "just a minute" and "can I ask you something" and thank you for sharing your thoughts and jokes. I am deeply indebted to you and I truly hope you will give me a chance to return the favor.

I want to thank Professor Reinhard Jahn and Professor Nils Brose for being on my PhD committee. Thank you for sharing your scientific experience and for giving helpful and critical advice.

I want to thank all the collaborators who aided in performing experiments either for this thesis or for the publications.

I want to thank all the lab members for making our lab a uniquely fun place to be. I will miss you and the nice and friendly atmosphere.

I want to thank Dr. Steffen Burkhardt, Kerstin Grüniger and Ivana Bacakova from the IMPRS Molecular Biology Program for their support and for creating such an excellent atmosphere.

I want to thank everyone at Boehringer Ingelheim Fonds, not only for the financial support, but also for organizing exciting retreats and for creating the BIF-spirit.

I want to thank all my teachers and instructors throughout kindergarten, school and university. Thank you for the patience, motivation and inspiration to teach and guide me - I know it wasn't always easy.

I want to thank all my family and friends for their support and friendship. I want to especially thank my parents for believing in me and for never letting me down, and my brother for being such an uncomplicated pal. Last but not least, I want to thank Juana for her love and support - thank you for walking with me. 


\section{ABSTRACT}

During communication at chemical synapses, small neurotransmitter-filled synaptic vesicles, housed in the pre-synaptic nerve terminal (bouton), fuse with the plasma membrane (exocytosis) and release their neurotransmitter content into the synaptic cleft. The fused synaptic vesicles are retrieved into the bouton (endocytosis), where they are refilled with neurotransmitter and re-integrated into the synaptic vesicle pool for further rounds of exocytosis (vesicle recycling). Through which pathways the synaptic vesicles recycle has been a matter of debate for more than three decades. Here we investigated the involvement of endosomes in the recycling of synaptic vesicles by resorting to a variety of novel imaging approaches.

First, we generated three novel exocytosis reporters by fusing a $\mathrm{pH}$-sensitive GFP variant (pHluorin) to the lumenal domain of the early endosomal fusion proteins Vti1a, Syntaxin 13 and Syntaxin 6. We compared the recycling of these reporters expressed in hippocampal neurons upon different stimulation paradigms and found them to be preferentially mobilized during readily releasable pool stimulation (RRP; 20 $\mathrm{Hz} / 2$ seconds). Second, using photo-oxidation electron microscopy of FM dye-labeled RRP vesicles, we observed a transient appearance of larger labeled organelles at 10 seconds after stimulation, indicative of endosomal fusion of endocytosed vesicles. We next used high-resolution thin-section STED microscopy and video-rate live-STED microscopy, to confirm that endocytosed RRP vesicles fuse with bona fide endosomes (identified by Rab5-GFP). Interestingly, stronger recycling pool stimulation $(20 \mathrm{~Hz} / 2$ seconds) did not seem to result in higher colocalization of vesicles and endosomes in these experiments. Third, we used expression of soluble Syntaxin 13 fragment to block formation of endosomal fusion complexes and therefore endosomal fusion of synaptic vesicles. Strikingly, this perturbation resulted in a drastic $\sim 60 \%$ decrease in RRP size, underscoring the importance of endosomal function for the RRP. Fourth, 2-color STED measurements indicated that synaptic vesicles persist as multi-component clusters in the plasma membrane, while comparison of the composition of general pool and recently endocytosed isolated synaptic vesicles revealed that endocytosis causes retrieval of "dirty" vesicles, thus necessitating endosomal sorting.

This is one of the first studies proposing a molecular and mechanistic determinant of RRP vesicles. We conclude that the RRP vesicles are maintained in a highly fusion-competent state by recycling through endosomes, making them the privileged vesicles drawn upon during physiological activity. 


\section{LIST OF FIGURES}

Figure 1-1: Different Models of SynAPtic Vesicle RECyCling 14

FiguRE 1-2: Synaptic Vesicle PoOLS 17

FiguRE 1-3: ENDOSOMAL TRAFFICKING ORGANELLES 20

FIGURE 2-1: DIAMINOBENZIDINE PHOTO-OXIDATION OF NEURONS LABELED WITH FM 1-43 44

FIGURE 2-2: RELATIVE DENSITY OF LABELED AND UNLABELED VESICLES AFTER PHOTO-OXIDATION. 44

FIGURE 3-1: PHLUORIN FUNCTION 55

FIGURE 3-2: THE ENDOSOMAL PHLUORINS DO NOT INHIBIT VESICLE RECYCLING 56

FIGURE 3-3: ENDOSOMAL PHLUORINS RECYCLE UPON ELECTRICAL STIMULATION 58

FIGURE 3-4: ENDOSOMAL FUSION AND BUDDING OF RRP VESICLES 61

FIGURE 3-5: QDOTS CONFIRM ENDOSOMAL FUSION AND BUDDING OF RRP VESICLES 62

FIGURE 3-6: EXPRESSION OF RAB5-GFP VARIANTS (WILD-TYPE OR Q79L) DOES NOT AFFECT ENDOSOMAL TRAFFICKING IN PC12 CELLS 64

FIGURE 3-7: SYNAPTOTAGMIN ANTIBODY UPTAKE INTO ENDOSOMES 66

FIGURE 3-8: DYNASORE INHIBITS SYNAPTIC VESICLE RECYCLING IN HIPPOCAMPAL NEURONS 67

FIGURE 3-9: ANALYSIS OF OVERLAP BETWEEN SYNAPTIC VESICLE MARKER AND ENDOSOMES 68

FIGURE 3-10: LIVE STED IMAGING OF SYNAPTIC VESICLES REACHING ENDOSOMES 69

FIGURE 3-11: BLOCKING ENDOSOMAL FUSION BY SOLUBLE SYNTAXIN 13 FRAGMENT 70

FIGURE 3-12: EFFECTS OF INHIBITING ENDOSOMAL FUSION ON SYNAPTIC VESICLE POOLS 71

FIGURE 3-13: QUANTIFICATION OF VESICLE POOLS UPON EXPRESSION OF SOLUBLE SYNTAXIN 13 FRAGMENT LACKING THE SNARE DOMAIN 73

FIGURE 3-14: MonTE CARLO MODELING OF VESICLE POOLS IN ABSENCE OF ENDOSOMAL SORTING 74

FIGURE 3-15: BLOCKING OF SURFACE SYNAPTOTAGMIN EPITOPES TOGETHER WITH INHIBITION OF ENDOCYTOSIS ALLOWS FOR SPECIFIC INVESTIGATION OF RECENTLY EXOCYTOSED VESICLES 75

FIGURE 3-16: SYNAPTOTAGMIN AND SYNAPTOPHYSIN REMAIN CO-CLUSTERED UPON EXOCYTOSIS 77

FIGURE 3-17: LABELING OF SMALL VESICLES BY ENDOCYTOSIS IN SYNAPTOSOMES 78

FIGURE 3-18: IN VITRO IMMUNOASSAY TO STUDY RECENTLY ENDOCYTOSED VESICLE COMPOSITION 79

FIGURE 3-19: ENDOCYTOSIS LEADS TO IMPURITIES IN SYNAPTIC VESICLE COMPOSITION 80

FIGURE 3-20: PLASMA MEMBRANE AND SYNAPTIC VESICLE MARKERS RESIDE IN DIFFERENT DOMAINS IN THE ENDOSOME MEMBRANE 82 


\section{LIST OF TABLES}

TABLE 2-1: PRIMARY ANTIBODIES USED THROUGHOUT THE STUDY 32

TABLE 2-2: BUFFERS AND SOLUTIONS___ 34

TABLE 2-3: FILTER-SETS USED FOR EPI-FLUORESCENCE MICROSCOPY ___ 35

TABLE 2-4: OLYMPUS OBJECTIVE SPECIFICATIONS ___ 35

TABLE 2-5: LASER LINES FOR CONFOCAL LASER SCANNING MICROSCOPY ___ 36

TABLE 2-6: PRIMERS FOR SNARE AMPLIFICATION___ 40

TABLE A-1: MATLAB FUNCTIONS USED FOR PHLUORIN ANALYSIS ___ 109

TABLE A-2: MATLAB FUNCTIONS USED FOR ANALYSIS OF ISOLATED VESICLE IMMUNOASSAYS___ 116 


\section{LIST OF ABBREVIATIONS}

AAA

AOBS

AOTF

APD

BAR domain

BSA

CCV

CTxB

DAB

$\mathrm{dF}$

DIV

FWHM

GAP

GDI

GEF

GFP

GPI

HEPES

Hsc70

IRES

KS-test

LDL

MBC

MVB

NSF

PBS

PCR
ATPases associated with diverse cellular activities

Acousto-optical beam splitter

Acousto-optical tunable filter

Avalanche photodiode

Bridging interactor/Amphiphysin/Recuced viability upon starvation domain

Bovine serum albumin fraction $\mathrm{V}$

Clathrin-coated vesicle

Cholera toxin beta subunit

Diaminobenzidine

Delta F; difference in fluorescence

Days in vitro

Full width at half maximum

GTPase activating protein

Guanine nucleotide dissociation inhibitor

Guanine nucleotide exchange factor

Green fluorescent protein

Glycosyl-phosphatidyl-inositol

4-(2-hydroxyethyl)-1-piperazineethanesulfonic acid

Heat shock protein cognate of $70 \mathrm{kDa}$

Internal ribosomal entry site

Kolmogorov-Smirnov test

Low-density lipoprotein

Methyl- $\beta$-cyclodextrin

Multi-vesicular body

$\mathrm{N}$-ethylmaleimide-sensitive-factor

Phosphate buffered saline

Polymerase chain reaction 


\begin{tabular}{l|l} 
PI(3)P & Phosphatidyl-inositol-3-phosphate \\
PMSF & Phenylmethanesulphonylfluoride \\
PMT & Photomultiplier tube \\
Rab & Ras-related in brain \\
ROI & Standard error of the mean \\
SEM & Soluble N-ethylmaleimide-sensitive-factor attachment protein receptor \\
SNARE & Synaptosome-associated protein of $25 \mathrm{kDa}$ \\
SNAP-25 & Antimulated emission depletion \\
STED & 2,2'-thiodiethanol \\
syt & Transferrin \\
TDE & Trans-Golgi network \\
Tf & Tetrodotoxin \\
TGN & Vesicle associated membrane protein 2 \\
TTX &
\end{tabular}




\section{INTRODUCTION}

\subsection{Pre-Synaptic Function}

Neurons are specialized cells that communicate with each other and muscle cells via cell-cell junctions, for which the term "synapse" (from the Greek 'syn' for 'together' and the Greek 'haptein' for 'to clasp') has been coined in 1897 by the British physiologists Sir Charles Scott Sherrington and Michael Foster (Foster and Sherrington, 1897), upon suggestion by the classicist Arthur Woolgar Verrall (Tansey, 1997). At some synapses, electrical impulses are propagated electrotonically; ions simply flow from the cytosol of the pre-synaptic cell to the cytosol of the post-synaptic cell via gap junctions (electrical synapse). Electrical synapses are typically found where high transmission speeds are of utmost importance, as for example in escape reflexes or in the retina of vertebrates. The high transmission speeds that can be achieved via gap junctions are also advantageous when synchronized activity of multiple cells is required: communication via electrical synapses can maintain synchronous firing of several neurons, and cardiomyocytes connected by gap junctions facilitate the simultaneous contraction of the heart muscle. Despite these important occurrences of electrical synapses and gap junctions, an indirect way of transmission is encountered much more often and takes place at the chemical synapse. (as detailed below; Kandel et al., 2000).

In the following sections, I will first cover the transmission at the chemical synapse in more detail, including molecular interactions required for exocytosis; I will then explain how the small membrane-bounded organelles characteristic for the function at the chemical synapse are recycled in the pre-synapse, followed by a discussion of what is known about the diversity of these organelles and the relevance of these differences. 


\subsubsection{Neurotransmitter release at the chemical synapse - exocytosis}

In comparison to the communication via gap junctions at electrical synapses, the processes at chemical synapses are characterized by disproportionately more complex mechanisms. The gap junctions, which allow direct passage of ions between cells and which are so characteristic for the electrical synapse, are not found at chemical synapses. The distinctive element of chemical synapses instead, is a specialized intracellular organelle termed "synaptic vesicle". Synaptic vesicles are small, neurotransmitter-filled, spherical organelles $(\sim 42 \mathrm{~nm}$ in diameter; Takamori et al., 2006) surrounded by a bilayer of lipid membrane that are housed in the pre-synaptic nerve terminal. As will be explained below, the synaptic vesicles fuse with the plasma membrane (exocytosis) at a specialized site of the pre-synaptic nerve terminal (active zone) and release their neurotransmitter content into the extracellular space that separates the pre- from the post-synaptic cell (synaptic cleft), so that the neurotransmitter can bind to post-synaptic surface receptors and elicit an electric response. Matching the importance and specialization of synaptic vesicles, they exhibit a unique lipid composition (in molar amounts: 40\% cholesterol, 22\% phosphatidylcholine, $14 \%$ phosphatidylethanolamine) and they are literally packed with numerous trans-membrane proteins and membrane-associated proteins (Takamori et al., 2006), several of which are directly or indirectly involved in mediating the fusion of the synaptic vesicles to the plasma membrane.

\subsubsection{Synaptotagmin - a calcium sensor for vesicle fusion}

Arrival of an action potential at the pre-synaptic nerve terminal causes voltagedependent calcium channels in the pre-synaptic membrane to open, leading to a local increase in calcium ion concentration from the high nanomolar to low micromolar range (Alberts, 2002; Randall et al., 2002). This rise in available calcium ions is sensed by an integral synaptic vesicle protein, Synaptotagmin I (Matthew et al., 1981; Perin et al., 1990). In rodents and humans, the Synaptotagmin family consists of 15 differentially expressed isoforms (Mittelsteadt et al., 2009). The Synaptotagmins contain an Nterminal trans-membrane domain and two C2-domains, providing calcium sensor functionality. Synaptotagmins I-III, V-VII and IX-X all contain five highly conserved aspartate residues (in both $\mathrm{C} 2$-domains) to bind $\mathrm{Ca}^{2+}$, and these isoforms were found to promote vesicle fusion in a $\mathrm{Ca}^{2+}$-dependent manner in vitro (Bhalla et al., 2008). The $\mathrm{Ca}^{2+}$ binding affinity of the Synaptotagmin I C2A domain is in the low micromolar range (Davletov and Sudhof, 1993), corresponding well to the free $\mathrm{Ca}^{2+}$ concentrations in the pre-synaptic cytosol upon action potential triggered opening of voltage-gated calcium 
channels. Upon binding of calcium ions to the C2-domains, Synaptotagmin I undergoes a conformational change, causing an increased affinity for phosphatidylserine (Davletov and Sudhof, 1993), a lipid mostly found in the cytoplasmic leaflet of the plasma membrane. This calcium-dependent interaction of the cytoplasmic domain of Synaptotagmin with the cytoplasmic leaflet of the plasma membrane has been interpreted as serving as a mechanism to associate the synaptic vesicle with the plasma membrane upon the arrival of an action potential, paving the way for the molecular interactions driving membrane fusion (Sudhof, 2004; Pang and Sudhof, 2010).

\subsubsection{SNAREs - the vesicle fusion machinery}

The fusion of the vesicle with the plasma membrane is controlled by the soluble $\mathrm{N}$ ethylmaleimide sensitive factor (NSF) attachment protein (SNAP) receptor (SNARE) proteins (Jahn and Scheller, 2006). All SNARE proteins share a conserved SNAREdomain, consisting of a helical stretch of about 60 amino acids (Weimbs et al., 1997; Fasshauer et al., 1998; Sutton et al., 1998), and most SNAREs contain a C-terminal transmembrane domain, anchoring the protein to the membrane, or at least a posttranslational modification, which allows loose association with the membrane. Furthermore, some SNAREs contain an N-terminal domain, which can have regulatory function for the SNARE-assembly (Burkhardt et al., 2008). SNARE-assembly starts by formation of a four-helix bundle of SNARE-domains of multiple SNAREs, residing on two different membranes (or organelles). This helical bundle zippers-up from the N- to Ctermini, forming a trans-SNARE-complex (the participating SNAREs are anchored to opposing membranes) and pulling the two membranes into close apposition, culminating in the merging of the two lipid bilayers. Characteristic for the resulting SNARE complex is a lining-up of amino acid residues at 16 conserved positions (layers) of each SNARE-domain (Jahn and Scheller, 2006). The original classification of the SNAREs was based on their presence on either target membranes (t-SNAREs) or vesicle membranes (v-SNAREs) (Sollner et al., 1993; Rothman, 1994). Although this classification is plausible for the classification of SNAREs involved in synaptic vesicle exocytosis, it falls short of providing a universal classification template; in homotypic fusion events, all participating SNAREs reside on the same type of organelle and the determination of the target membrane would appear rather arbitrary. Subsequent comparison of various SNARE domains revealed that they can be subdivided into two types, based on a single conserved amino acid residue at the zero-layer, in the middle of the SNARE-motif: while the R-SNAREs share a conserved arginine in this position, the QSNAREs contain a conserved glutamine (Fasshauer et al., 1998). 
In the case of synaptic vesicle exocytosis, the four helices are contributed by the synaptic vesicle protein Synaptobrevin 2 (also know as vesicle associated membrane protein 2 [VAMP2]; v-/ R-SNARE), by the plasma membrane protein Syntaxin 1 (t-/ QSNARE) and by the plasma membrane-associated (via palmitoylation) protein synaptosome-associated protein of $25 \mathrm{kDa}$ (SNAP-25; t-SNARE containing 2 Q-SNARE domains). As Synaptobrevin is anchored to the vesicle membrane and Syntaxin 1 and SNAP-25 are anchored to (or associated with) the plasma membrane, the zippering of the SNARE domains pulls the vesicle membrane and the plasma membrane together. Besides its interaction with the cytoplasmic leaflet of the plasma membrane, Synaptotagmin I was also found to interact with Syntaxin 1 (Bennett et al., 1992) and SNAP-25 (Schiavo et al., 1997) and is thought to promote and regulate the fusion reaction. After successful membrane merger, the cis-SNARE complexes (with all SNAREs now residing in the same continuous membrane) are resolved by the AAA (ATPases with diverse cellular activities)-ATPase NSF and its cofactor $\alpha$-SNAP, so that the SNAREs are available for future rounds of fusion (Jahn and Scheller, 2006).

Upon merger of the lipid bilayers of the vesicle and the plasma membrane, the vesicle lumen becomes continuous with the extracellular medium; due to the steep concentration gradient of neurotransmitter between vesicle lumen and extracellular medium, it diffuses across the synaptic cleft and binds to post-synaptic surface receptors, which directly or indirectly elicit an electrical response (Kandel et al., 2000).

In summary, the electrical signal arriving at the pre-synaptic nerve terminal as an action potential has traversed the synaptic cleft by means of a chemical signal, which has been re-coded at the surface of the post-synaptic cell, again into an electrical signal. In contrast to the electrical synapse, where electrical signals are relayed from cell to cell as graded potentials, the signal conversions at a chemical synapse significantly slow down transmission ( $\sim 0.2 \mathrm{~ms}$ vs. $\sim 2 \mathrm{~ms}$, respectively). Nevertheless, the chemical synapse allows for various ways of fine-tuning and regulation of speed and strength of a synapse, making the chemical synapse the prototype for the majority of synapses in the central nervous system (Kandel et al., 2000). 


\subsubsection{Synaptic vesicle recycling}

What happens to the synaptic vesicle components in the plasma membrane upon exocytosis?

One would expect the pre-synaptic nerve terminal to grow constantly as vesicles are continually added to the plasma membrane during activity. This, however, has been known for decades not to be the case (Heuser and Reese, 1973). There is a compensatory mechanism retrieving membrane material from the plasma membrane into the nerve terminal (endocytosis), eventually forming new fusion-competent synaptic vesicles (recycling). Several pathways of synaptic vesicle recycling have been described in the past (see reviews by: Sudhof, 2004; Rizzoli and Jahn, 2007), and they are briefly described below (see also Figure 1-1).

A

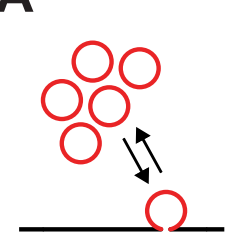

B
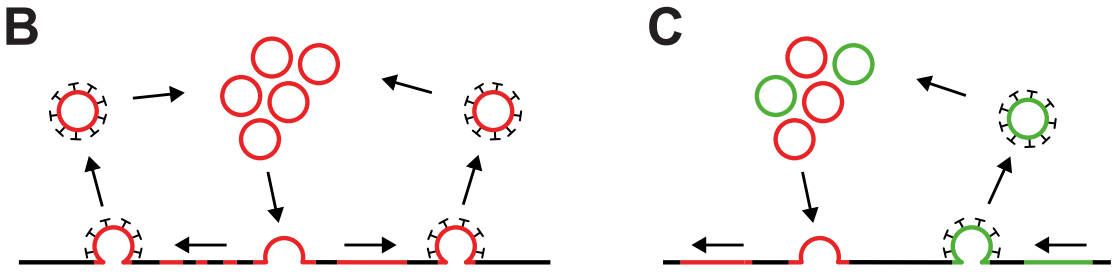

D

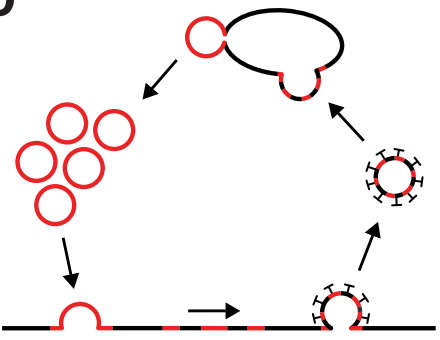

E

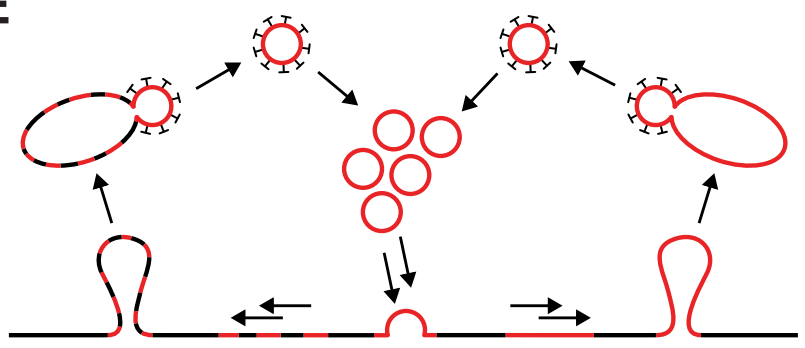

Figure 1-1: Different Models of Synaptic Vesicle Recycling

(A) Kiss-and-run. Vesicles (red) fuse transiently with the plasma membrane (black) by opening of a small fusion pore and recycle locally by pinching off.

(B) Clathrin-mediated endocytosis. Vesicles collapse completely into the plasma membrane. The vesicle and plasma membrane material could either mix (left) or the vesicle components could remain as a patch in the plasma membrane (right). The synaptic vesicles are endocytosed by clathrin-mediated endocytosis, via clathrin-coated pits and vesicles, and integrate into the vesicle cluster after uncoating.

(C) Readily retrievable vesicles. Vesicles recycle as in classical clathrin-mediated endocytosis, except that the vesicle fusing (red) and the vesicle being endocytosed (green) are not identical.

(D) Endosomal recycling. Vesicles undergo full-collapse fusion but mixing with the plasma membrane leads to uptake of mixed vesicle material by clathrin-mediated endocytosis. After uncoating, the retrieved vesicles fuse to an endosome, from which new synaptic vesicles bud.

(E) Bulk endocytosis. During heavy activity, fusion of many vesicles causes saturation of classical endocytosis. Plasma membrane invaginations form cisternae of mixed plasma membrane / vesicle material (left) or cisternae of only vesicle material (right), from which clathrin mediated budding forms new synaptic vesicles. 


\subsubsection{Schematics modified from (Rizzoli and Jahn, 2007).Kiss-and-run}

A rather recently described synaptic vesicle recycling pathway has been named "kissand-run" (see Figure 1-1 A), in allusion to the fact that it only involves transient fusion of the synaptic vesicle to the plasma membrane by opening of a relatively small fusion pore, followed by local recycling of the vesicle (Ceccarelli et al., 1973; Fesce et al., 1994). This recycling mode is thought to not involve mixing of vesicle and plasma membrane material, which would in principle allow the vesicles to recycle indefinitely. Among the theoretical advantages of this recycling model are (1) the potentially fast recycling speed that would allow fast reuse of vesicles (due to the simple opening and closing of a fusion pore), (2) the inbuilt maintenance of the vesicle composition that would ensure fusion-competency of the vesicles (due to the absence of intermixing of vesicle and plasma membrane), and (3) the low energy expenditure (due to the small recycling machinery and the superfluity of re-establishing the vesicle composition after intermixing in the membrane). Despite these potential benefits entailed by kiss-and-run, the experimental evidence for this pathway remains disputed (see below), also because no molecular determinants of kiss-and-run have been described to date.

\subsubsection{Clathrin-mediated endocytosis}

The classical pathway of synaptic vesicle recycling involves endocytosis of synaptic vesicle material via clathrin-coated pits and vesicles (see Figure 1-1 B). This welldescribed pathway involves binding of stonin 2 to both AP2 and Synaptotagmin I, recruiting clathrin to form coated pits containing synaptic vesicle material (Poskanzer et al., 2003; Walther et al., 2004; Diril et al., 2006). Clathrin was first described by this name in the late 1970's (Pearse, 1976) as a conserved coat protein of vesicles in brain, adrenal medulla and a lymphoma cell line. The polyhedral clathrin coats are formed by interaction of multiple clathrin triskelia that are composed of three clathrin heavy chains and three clathrin light chains each (Edeling et al., 2006).

In contrast to the absence of knowledge about the molecular players in kiss-andrun, the clathrin machinery has been characterized in much detail, so that many direct and indirect clathrin interaction partners and their functions are known. Among these are the adaptor proteins AP180 and AP2 (Hao et al., 1999), the BAR (Bridging interactor/Amphiphysin/Reduced viability upon starvation) domain-containing (Peter et al., 2004; Frost et al., 2009) membrane curvature-inducing proteins Amphiphysin (Lichte et al., 1992; David et al., 1996) and Endophilin (Micheva et al., 1997; Schmidt et al., 1999), the GTPase Dynamin that promotes the pinching-off of the coated vesicle (Sweitzer and Hinshaw, 1998), as well as the chaperone Hsc70 (heat shock protein 
cognate of $70 \mathrm{kDa}$; also known as uncoating ATPase; Paddenberg et al., 1990) and the phosphoinositide phosphatase Synaptojanin (McPherson et al., 1994; Ramjaun and McPherson, 1996; Haffner et al., 2000), both of which are involved in the shedding of clathrin coats.

In the basic variant of clathrin-mediated endocytosis, the uncoated vesicles could be ready for a new round of fusion simply by refilling with neurotransmitter (Takei et al., 1996), which is generally achieved by pumping protons into the vesicle lumen by the action of the vacuolar $\mathrm{H}^{+}$-ATPase, followed by passive transport of the respective neurotransmitter into the vesicle lumen via integral transmitter transporters (Südhof et al., 2008). Alternative variants of clathrin-mediated recycling include (1) the concept of "readily retrievable" vesicles (Fernandez-Alfonso et al., 2006; Wienisch and Klingauf, 2006), which allows shorter endocytosis lag-times by internalizing a preformed coated pit rather than the recently exocytosed vesicle (see Figure 1-1 C), and (2) the fusion of internalized vesicles to an endosomal compartment (Heuser and Reese, 1973 ) to sort vesicle components after intermixing in the plasma membrane (detailed in section 1.1 below; see also Figure 1-1 D).

\subsubsection{Bulk endocytosis}

Another pathway that has been described to be functional for synaptic vesicle recycling is "bulk endocytosis" (Gennaro et al., 1978; Miller and Heuser, 1984). The hallmark of this mechanism is the clathrin-independent formation of large plasma membrane invaginations, which eventually pinch-off to form intracellular cisternae (see Figure 1-1 E). This pathway has been described to be triggered during heavy exocytotic activity (Clayton et al., 2008), when the capacity of classical clathrin-mediated endocytosis is surpassed (see Royle and Lagnado, 2003). This way, the excessive fusion of vesicles is not accompanied by growth of the nerve terminal, and budding from the internal cisternae forms new vesicles. In view of the fact that the internal cisternae represent a plasma membrane-derived compartment that might even remain connected to the plasma membrane, it is not surprising that the subsequent budding of small vesicles from these bulk invaginations requires clathrin and its machinery, in a process that is essentially endocytosis from the (internalized) plasma membrane. 


\subsubsection{Synaptic vesicle pools}

Despite the ongoing discussion about the recycling pathways taken by synaptic vesicles, there is general consensus regarding the existence of several different synaptic vesicle pools - functionally defined subsets of synaptic vesicles (see Figure 1-2). Synaptic vesicle pools have been described in various organisms and are typically classified as three separate pools (see review by Rizzoli and Betz, 2005).

First, the readily releasable pool (RRP) of vesicles consists of those few vesicles being most fusion-competent; upon arrival of action potentials at the nerve terminal, the RRP vesicles (corresponding to the morphologically docked vesicles; Schikorski and Stevens, 2001) first undergo fusion at the active zone. For the preparation of choice in this study, cultured neurons from the rat hippocampus, this vesicle pool only contributes $\sim 5 \%$ of all synaptic vesicles (about 10 out of 200; Schikorski and Stevens, 1997). In vitro, this pool of vesicles can be mobilized by a brief high-frequency electrical stimulus of 40 action potentials (20 Hz for 2 seconds; Murthy and Stevens, 1998, 1999; Schikorski and Stevens, 2001) or by brief exposure to hypertonic sucrose solution (Rosenmund and Stevens, 1996). As each vesicle only shows a limited release probability, the overall release probability of a synapse has been proposed to depend on the size of the RRP - the more vesicles are docked and available for exocytosis, the higher the probability to release one of them (Murthy et al., 2001).

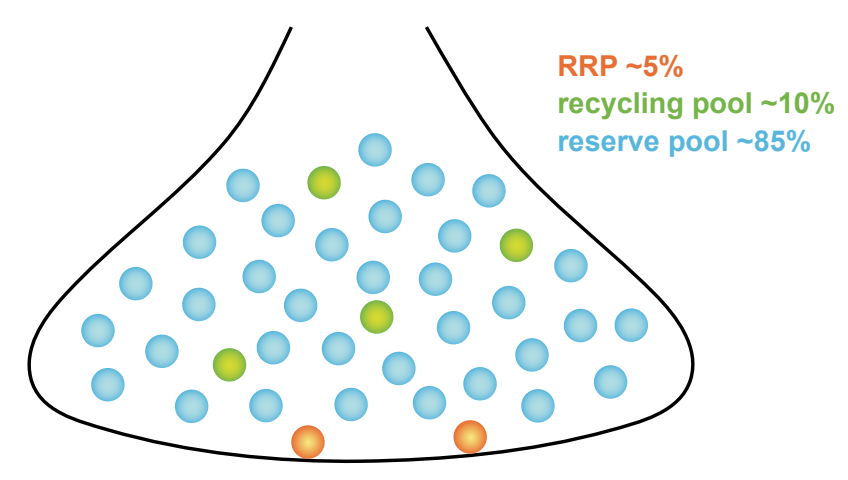

Figure 1-2: Synaptic Vesicle Pools

Three classical synaptic vesicle pools in the pre-synaptic nerve terminal of cultured hippocampal neurons. The readily releasable pool (RRP; orange) vesicles are docked at the active site and constitute only about $5 \%$ of all vesicles. The recycling pool (green; $~ 10 \%$ of all vesicles) and the reserve pool (blue; $\sim 85 \%$ of all vesicles) are spatially intermixed. (compare Rizzoli and Betz, 2005) 
Second, the recycling pool contributes $\sim 10 \%$ of all vesicles (about 20 vesicles) and it recycles at prolonged electrical stimulation $(20 \mathrm{~Hz} / 30 \mathrm{~s})$ in vitro (Murthy and Stevens, 1999; Harata et al., 2001b; Harata et al., 2001a). These vesicles are thought to recycle repeatedly and intermix with the RRP on a relatively fast timescale; whether there is any difference between RRP and recycling pool vesicles in terms of molecular composition remains to be determined (see Rizzoli and Betz, 2005).

Third, the reserve pool of vesicles is reluctant to release in cultured hippocampal neurons, even after prolonged high-frequency stimulation or brief depolarization with high- $\mathrm{K}^{+}$solution (Harata et al., 2001b; Harata et al., 2001a). Although these vesicles can be released at prolonged high-frequency stimulation in other preparations, including the larval Drosophila neuromuscular junction and the frog neuromuscular junction (Delgado et al., 2000; Richards et al., 2000) it seems that this pool of vesicles is not recruited under physiological conditions. For example, the reserve pool mobilization observed in the temperature sensitive Drosophila mutant shibire (which is unable to endocytose vesicles at non-permissive temperature; Grigliatti et al., 1973; Koenig and Ikeda, 1989; van der Bliek and Meyerowitz, 1991) upon excessive stimulation at $10 \mathrm{~Hz}$ for 5 minutes at non-permissive temperature (Delgado et al., 2000) was certainly evoked by an extreme situation of vesicle depletion, which might never be naturally encountered. It remains to be noted that the role of the reserve pool in vivo has not been determined conclusively - by choosing sufficiently strong stimulation paradigms, the reserve pool can be recruited to release in several preparations, but whether this is of any physiological relevance is not described.

Although the functional differences between synaptic vesicle pools have been excessively studied, not much is known about the root of these functional differences. The group of Kidokoro has suggested that the pools are spatially segregated in Drosophila, which could explain the differences in release probability (Kuromi and Kidokoro, 2003). According to their observations, the exo/endo cycling pool (the equivalent of a combined RRP and recycling pool) is located in proximity to the active sites, where synaptic vesicles fuse with the plasma membrane, while the reserve pool is located in the center of the terminals. This view, however, is not in agreement with findings also by the same group, showing that the terminal centers are normally devoid of vesicles in this preparation (Atwood et al., 1993; Kuromi et al., 2004). In addition, this view has been challenged by recent studies showing that the functional vesicle pools are spatially intermixed (Akbergenova and Bykhovskaia, 2009; Denker et al., 2009). These latter findings support the notion that the functional vesicle pools might be made up of molecularly non-identical vesicles. Even though the composition of a typical synaptic 
vesicle has been determined in the exhaustive study by Jahn and coworkers (Takamori et al., 2006), this composition 'only' represents a biochemical average of a population of purified vesicles. It might well be that there are several sub-populations of vesicles diverging more or less drastically from the composition of this average synaptic vesicle. Such differences in vesicle composition might account for the functional differences between the vesicle pools - nevertheless, we are currently not in the position to make such classifications. 


\subsection{Endosomal Trafficking}

The endosomal system consists of several more or less well defined intracellular organelles associated with the trafficking of various cargoes between the plasma membrane and the trans-Golgi network (TGN). Below follows a short description of the characteristic properties and functions of the organelles in the endosomal system.

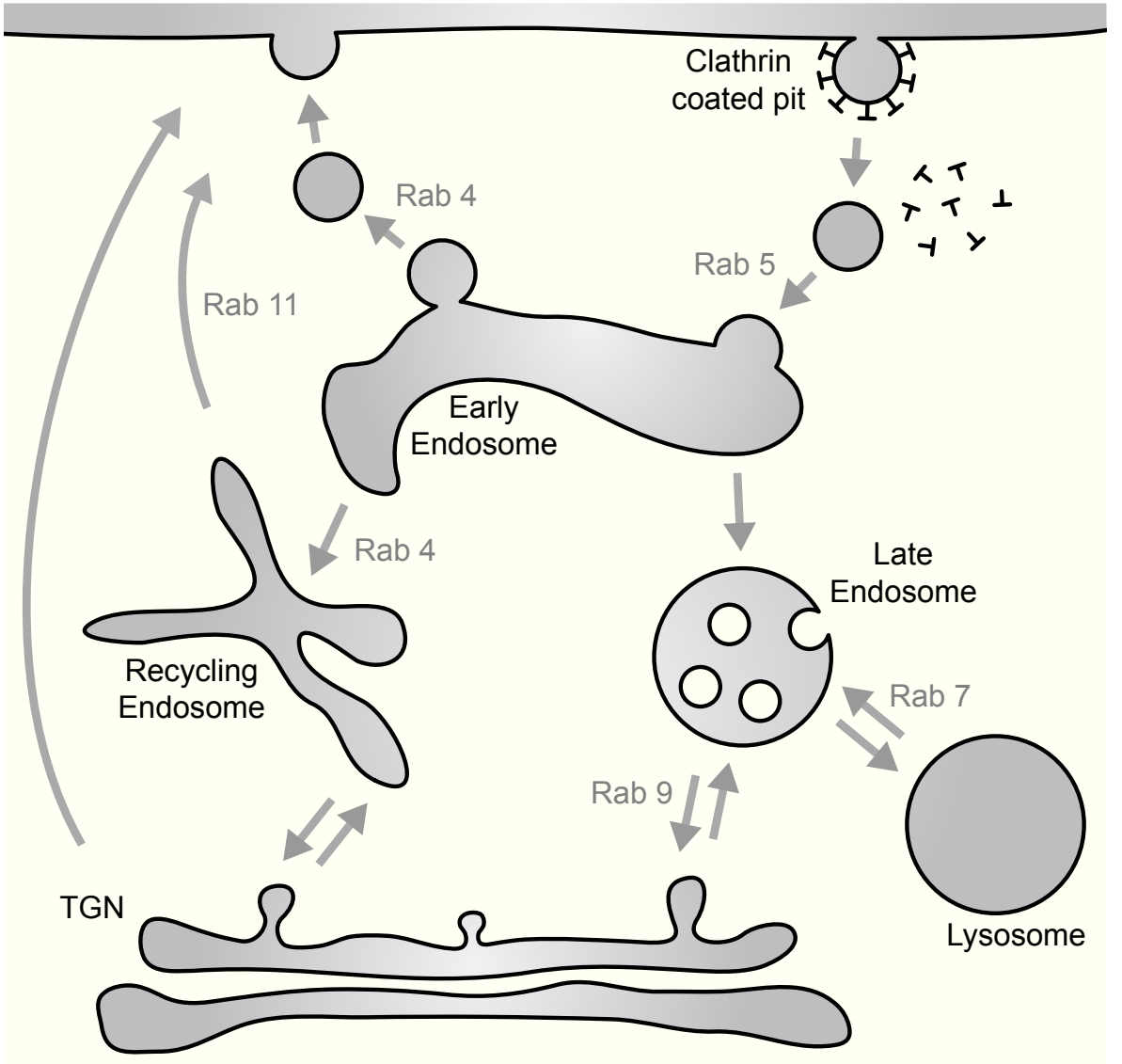

Figure 1-3: Endosomal Trafficking Organelles

Schematic representation of the endosomal compartments as outlined in the main text. Note that the various trafficking steps typically involve specific Rab (Ras-related in brain) proteins to recruit further factors required for fusion. 


\subsubsection{The endosomal compartments}

The endosomal system consists of various organelles and interfaces to the secretory pathway (see Figure 1-3). In brief, vesicles endocytosed via clathrin-coated pits are delivered to the early (or sorting) endosome, which serves as the major trafficking hub in the endosomal system. From here, cargoes can be rerouted directly to the cell surface in a fast recycling pathway, they can take a slow recycling pathway via the recycling endosome back to the surface, or they can move towards the degradative pathway by reaching late endosomes and lysosomes. Both the recycling endosome and the late endosome also exchange material with the TGN, which allows bi-lateral traffic between the endosomal system and the secretory compartments.

The endosomal compartments and the traffic between them are discussed in more detail in the following sections.

\subsubsection{Early endosome}

The early endosome is the first organelle receiving material taken up into the cell by endocytosis. It acts as a sorting hub, directing incoming soluble and membrane-bound cargoes towards their proper destinations. For example, incoming nutrients will be directed towards the lysosome (see below) or surface receptors will be sent back to the plasma membrane by means of secretory vesicles.

The molecular components giving identity to the early endosome are understood relatively well. Small GTPases of the Rab (Ras-related in brain; Touchot et al., 1987) family have been identified as major regulators of tubulo-vesicular transport in eukaryotic cells (Stenmark and Olkkonen, 2001; Stenmark, 2009). As all small GTPases, Rabs cycle between two states. When in the GTP-bound form, Rabs are in their active conformation and are anchored to membranes via the exposed geranylgeranylanchor. In this active state, the Rabs recruit Rab effectors and promote cellular processes like vesicle transport, uncoating, budding and fusion. Due to their intrinsic GTPase activity, which is further enhanced by interaction with a GTPase activating protein (GAP), GTP is hydrolyzed to GDP and inorganic phosphate. This hydrolysis effects a conformational change and inactivation of the Rab, also causing it to dissociate from the membrane. The lipophilic geranylgeranyl-anchor of the soluble Rab is sequestered by the guanine nucleotide dissociation inhibitor (GDI). For the reactivation of the Rab, the GDP has to be substituted for GTP by the guanine nucleotide exchange factor (GEF). 
The Rab protein Rab5 has been identified on early endosomes (Chavrier et al., 1990) and synaptic vesicles (Takamori et al., 2006) and has been implicated in clathrinmediated endocytosis and endosomal fusion (Stenmark, 2009). Besides Rab5, early endosomes are known to be enriched in PI3-kinase and the phospholipid phosphatidylinositol-3-phosphate (PI3P). This endosomal lipid mediates the recruitment of other regulatory proteins like the early endosome antigen 1 (EEA1). In fact, EEA1 is recruited by means of coincidence detection: it binds to PI3P via its FYVE-domain and to Rab5 via $\mathrm{C}$ - and N-terminal Rab binding domains. Thus, EEA1 serves as a tethering factor for fusion, possibly also between Rab5 positive organelles (Stenmark, 2009). As for synaptic vesicle exocytosis, endosomal fusion requires formation of a SNARE complex, in this case including the SNAREs Vti1a, Syntaxin 13, Syntaxin 6 and VAMP4 (Rizzoli et al., 2006).

Although Rab5 is so intricately involved in early endosome dynamics, it is not the only Rab found on this organelle - early endosomal microdomains enriched in Rab4 have been identified to be involved in the direct recycling of cargoes back to the plasma membrane (Stenmark, 2009). It becomes more and more apparent that the Rabome plays a key role in determining the functional repertoire of organelles (Gurkan et al., 2005; Stenmark, 2009).

\subsubsection{Recycling endosome}

Rather than being recycled directly back to the plasma membrane from the early endosome (fast recycling route), some cargoes take an indirect route via the recycling endosome. As is the case with Rab5 and Rab4 in early endosomes, the recycling endosome is characterized by the presence of several Rab microdomains. Besides the Rab11 domains involved in recycling material back to the plasma membrane, the recycling endosome contains Rab4 domains receiving material from the early endosome, as well as tubular compartments enriched in Rab10, Rab11, Rab22A and Rab35 (Grant and Donaldson, 2009; Stenmark, 2009). The recycling endosome also directs some cargoes to the TGN, which is a common route taken by some toxins, like the cholera toxin beta subunit (CTxB; Nichols and Lippincott-Schwartz, 2001; Lencer and Tsai, 2003)

\subsubsection{Late endosome and lysosome}

While the early endosome is clearly specialized in the sorting of various cargoes to and from multiple destinations in the cell, the function of the late endosome is tailored more towards the degradative pathway. How the late endosome is formed is still a subject of debate: one hypothesis suggests that late endosomes are formed from carrier vesicles 
forming a stable late endosome, while the second model suggests that late endosomes derive directly from the remnants of early endosomes via endosome maturation (Clague, 1998). In line with the latter model, it was discovered that Rab5 positive early endosomes undergo conversion to a Rab7 positive late endosome (Rink et al., 2005; Poteryaev et al., 2010). Interestingly, late endosomes exhibit additional Rab9 domains that have been implicated in the exchange of material with the TGN (Stenmark, 2009). The distinct morphological feature of late endosomes is the existence of internal vesicles, which originate by invagination and budding of late endosomal surface membrane. These internal vesicles have given rise to the term multi-vesicular body (MVB) as a synonym for the late endosome. Late endosomes mainly deliver transmembrane proteins and soluble cargoes to the lysosome for degradation, but some trans-membrane proteins can also be recycled back to the plasma membrane (Lodish, 2003).

Although formally not part of the endosomal system, the lysosome is so tightly connected with the late endosome that it shall not remain unmentioned here. The transport between late endosome and lysosome is governed by Rab7, one of the major Rabs on late endosomes (Stenmark, 2009). As its name suggests, the lysosome is in charge of breaking down macromolecules by means of acid-activated hydrolases; material reaching the lysosome is destined for degradation and will be used to provide molecular building blocks for biosynthetic pathways (Lodish, 2003). 


\subsubsection{Sorting of cargo}

The endosome directs various cargoes to their various destinations, but how is the sorting achieved?

A well-understood example of endosomal sorting is the recycling of cell surface receptors, which I will briefly describe by the example of the low-density lipoprotein (LDL) receptor. The LDL receptor is expressed on the cell surface of many cells and contains a short C-terminal cytosolic segment that is connected to a longer $\mathrm{N}$-terminal exoplasmic part via a single trans-membrane helix; this exoplasmic part is made up of a $\beta$-propeller domain and a ligand-binding arm (Yamamoto et al., 1984; Lodish, 2003). The binding arm is characterized by seven imperfect cystein-rich repeats and interacts with the apoB-100 protein, which is part of LDL particles.

The LDL particle consists of a core of cholesteryl-esters and triglycerides ( $90 \%$ cholesteryl-esters) that is surrounded by a monolayer lipid membrane consisting of phospholipids and cholesterol. The single copy of the apoB-100 protein wrapped around this lipid particle mediates detection by the binding arm of the LDL receptor. Containing high amounts of cholesterol, the function of LDL particles is to deliver cholesterol to the cells (mostly hepatocytes) via the bloodstream. The binding of the LDL receptor's binding arm to apoB-100 only constitutes the first step in attaining this goal; subsequently, the receptor-LDL complex needs to be internalized by the cell. This uptake is promoted by a short amino acid sorting sequence (NPxY, where $\mathrm{x}$ can be any amino acid) in the cytoplasmic domain of the receptor, which allows interaction with AP2 and hence enriches the receptor complexes in clathrin-coated pits. After uptake into endocytic vesicles, the receptor-LDL complexes are delivered to the early endosome, where some fast recycling cargoes are sorted into budding vesicles, some slow recycling cargoes are targeted to the recycling endosome, and yet other cargoes are directed towards the degradative pathway (see above). As the cholesterol from the LDL particles has to be released by hydrolysis, the receptor-LDL complexes take the latter pathway towards the late endosome and lysosome. The maturation of the early endosome to a late endosome is not only characterized by Rab conversion (see above), but most notably also by acidification of the lumen. Due to the more acidic $\mathrm{pH}$ in the late endosome, the LDL particle dissociates from the LDL-receptor and is released into the endosome lumen. From this point on the paths of the receptor and its cargo separate: the former is recycled back to the plasma membrane, the latter progresses to the lysosome for degradation. 
Although one might expect sorting sequences on both apoB-100 and on the LDLreceptor responsible for their differential sorting, no such signals have been identified. It seems that the default pathway for surface receptors is to be delivered back to the plasma membrane, without the requirement of any particular signal. Conversely, the receptor needs to be explicitly marked for lysosomal degradation (for example by ubiquitylation; Katzmann et al., 2002), leading to its packaging into internal lateendosomal vesicles and progression to the lysosome.

How is sorting achieved in the absence of a sorting signal? A proposed mechanism, 'iterative, geometry-based sorting' (Maxfield and McGraw, 2004; Cullen, 2008), takes advantage of the geometry of endosomal organelles and their surface-tovolume ratios. By forming rather long, tubular membrane protrusions from the endosome (resembling a water-filled latex-glove), these protrusions will automatically enrich in the receptor compared to the body of the endosome. Conversely, the body of the endosome will be relatively scarce in receptor proteins but rich in soluble content because of its relatively large volume. Although seemingly unspecific, such a mechanism allows the endosome to recycle receptor proteins to the plasma membrane while keeping soluble cargoes within the endo-lysosomal compartments. As mentioned above, retention of membrane proteins in the endosomal system might require additional sorting signals and protein-protein interactions.

Another mechanism to separate and sort membrane proteins, namely the formation of membrane rafts or domains, has been introduced about two decades ago (Simons and van Meer, 1988; Brown and Rose, 1992; Simons and Ikonen, 1997; Simons and Toomre, 2000). This mechanism could be seen as an extension of the geometrybased model, rather than a replacement: it allows the separation of differently composed membrane patches and lumenal content, as opposed to only separating surface from volume. Lipid rafts are small ( 10-200 nm) domains enriched in cholesterol, sphingolipids and glycosyl-phosphatidyl-inositol (GPI) -anchored proteins. Since the original proposition of lipid rafts, the formation of domains and the clustering of proteins has become a frequently observed motif. Proteins identified to reside in such clusters are, among others, amyloid beta (Lee et al., 1998), SNARE proteins (Lang et al., 2001; Chamberlain and Gould, 2002) and the synaptic vesicle protein Synaptotagmin 1 (Willig et al., 2006). The potential relevance of protein-lipid-domains for endosomal sorting is evident: if a group of (for example) surface receptors remains clustered after endocytosis, they can be easily returned to the plasma membrane as one patch in a single step. 


\subsection{Endosomes and Synaptic Vesicles}

As described above, the endosomal system is well established as an intracellular sorting system connecting various intracellular organelles; it maintains the balance of in- and outbound cargoes and sorts lipids and proteins to their proper destinations within the cell. In this section I will discuss the current status about the involvement of endosomes in synaptic vesicle recycling.

\subsubsection{Are endosomes involved in synaptic vesicle recycling?}

The chemical synapse constitutes a cellular compartment specialized in the release of neurotransmitter by means of vesicle exocytosis. Even if a small central nervous system synapse, like those of cultured hippocampal neurons, only recycled a single vesicle per second (which seems to be a good estimate, considering average firing rates of about 5 Hz; Evarts, 1964; Green and Gillette, 1982), it would still have to deal with almost 4,000 vesicles exo- and endocytosing per hour. Considering that activity is not constant over time, there should be transient timeframes of substantially higher recycling rates. Such high membrane turnover rates likely pose a challenge for the cell with respect to maintaining the molecular composition/identity of its organelles. Particularly the highly specialized composition of the synaptic vesicles (Takamori et al., 2006) is crucial for the proper functioning of synaptic transmission: vesicle exocytosis would eventually cease, if the synaptic vesicles slowly lost their copies of, for example, the exocytotic SNARE Synaptobrevin (of which they contain about 70 molecules on average; Takamori et al., 2006 ) in the plasma membrane during repeated rounds of fusion. There is indeed some evidence that the molecular integrity of synaptic vesicles is maintained throughout their lifetime: vesicle components do not seem to accumulate in the plasma membrane (Valtorta et al., 1988) and synaptic vesicles do not seem to get enriched in plasma membrane material upon continuous recycling (Mitchell and Ryan, 2004).

As described above, the ubiquitous constitutive clathrin-mediated endocytosis pathway delivers membrane-bound proteins and soluble content to early endosomes by default. There is a striking common feature with synaptic vesicle recycling here: clathrin-mediated endocytosis has been described to retrieve synaptic vesicles into the nerve terminal (see for example Miller and Heuser, 1984; and the review by Rizzoli and Jahn, 2007). Although it seems almost natural, in view of this parallel, to propose the involvement of endosomes also in the recycling of synaptic vesicles, the situation in the highly specialized compartment of the pre-synapse might not be as straight forward. But indeed an endosomal recycling model has been proposed as early as 1973 (Heuser and 
Reese, 1973). This proposition was based on the appearance of coated pits and vesicles that coalesced to form large internal cisternae upon heavy stimulation of frog neuromuscular junctions $(10 \mathrm{~Hz} / 15$ minutes). In similar experiments, Mauro and collaborators (Ceccarelli et al., 1973) argued against this hypothesis, as they observed no depletion of synaptic vesicles, no increase in the number of coated vesicles and no appearance of larger organelles. These latter results marked the beginning of the emerging model of fusion characterized by only transient fusion of a vesicle to the plasma membrane via opening of a small fusion pore (later termed "kiss-and-run"; Fesce et al., 1994; see also review by Valtorta et al., 2001). The original Heuser and Reese model was later adapted to accommodate conflicting results: synaptic vesicles could either be formed simply by uncoating of endocytosed clathrin coated vesicles (CCVs) or by budding from internal cisternae originating from bulk endocytosis (Gennaro et al., 1978; Miller and Heuser, 1984). The coated vesicles connected to internal cisternae that Heuser and Reese had observed were now interpreted as coated vesicles budding from, rather than fusing to, these cisternae; also more recent studies have reported bulk endocytosis (Takei et al., 1996; Clayton and Cousin, 2008; Clayton et al., 2008; Cheung et al., 2010; Clayton et al., 2010; Glyvuk et al., 2010), while the endosomal model did not receive much further attention.

\subsubsection{Evidence for the endosomal model}

In part because of this negligence of the endosomal sorting model in synaptic vesicle recycling, the evidence in support of this model is relatively scarce and most of it did not remain uncontested. As mentioned before, early endosomal markers including Rab5 and endosomal SNAREs have been identified on synaptic vesicles (Fischer von Mollard et al., 1994; Antonin et al., 2000; Rizzoli et al., 2006; Takamori et al., 2006); however, synaptic vesicles also contain a plethora of other (not exocytosis-related) Rabs and SNAREs (Takamori et al., 2006), allowing the interpretation that their composition might simply not be controlled exceedingly strict. Additionally, the endosomal lipid PI3P was found to recycle together with synaptic vesicles in the Drosophila neuromuscular junction (Wucherpfennig et al., 2003), indicative of early endosomal recycling; but the existence of such an endosomal compartment in mammalian neurons has been contested (Murthy and Stevens, 1998). Furthermore, although purified synaptic vesicles from mammalian synaptosomes (isolated synapses) can fuse with bona fide sorting endosomes in vitro, the observed fusion rates are extremely low (Rizzoli et al., 2006), questioning the physiological relevance of these in vitro experiments. Finally, synaptic vesicle recycling is generally affected (at least to some extent) by perturbations of endosomal proteins 
including Rab5 (de Hoop et al., 1994; Wucherpfennig et al., 2003), PI3-kinase (Rizzoli and Betz, 2002), the adaptor protein AP3 (Voglmaier et al., 2006) and the adaptor protein $\sigma 1 \mathrm{~B}$-adaptin (thought to participate in sorting endosome dynamics; Glyvuk et al., 2010); however, none of these approaches targeted exclusively the synaptic vesicleendosome interaction, possibility leading to of off-target effects.

\subsubsection{Could kiss-and-run eliminate the requirement of endosomal sorting?}

Especially in recent years, the conceptually much simpler kiss-and-run recycling model was placed in the center of attention. Is it possible that this recycling mode represents the solution of the chemical synapse to deal with the high turnover-rate of an organelle specialized for regulated exocytosis? Could the existence of kiss-and-run explain why the otherwise omnipresent role of early endosomes in sorting endocytosed vesicles might not be functioning in synaptic vesicle recycling?

There has been a plethora of reports on the existence of the kiss-and-run model (Klingauf et al., 1998; Sun et al., 2002; Gandhi and Stevens, 2003; Harata et al., 2006; He et al., 2006; Zhang et al., 2009), but due to technical challenges, direct evidence for the existence of kiss-and-run is still missing. Although electron microscopy can readily provide the required resolution to identify individual vesicles and even fusion pores (typically $\sim 10-20 \mathrm{~nm}$; Torri-Tarelli et al., 1985), it does not provide the necessary temporal dynamics. As classical full collapse fusion also begins with the opening of a small fusion pore, observing such a pore in electron microscopy cannot be interpreted as evidence for kiss-and-run. Conversely, the temporal resolution provided by the combination of capacitance measurements and fusion pore conductance measurements allowed the calculation of the size of the kiss-and-run fusion pore $(>2.3 \mathrm{~nm}$; He et al., 2006), but this very indirect way of detecting kiss-and-run cannot exclude the possibility of capacitance flickers arising from the fusion of one vesicle and the subsequent endocytosis of a different one (readily retrievable vesicles; Wienisch and Klingauf, 2006). Similarly, the observation of fast sub-second endocytosis in capacitance measurements was attributed to fast kiss-and-run recycling (Sun et al., 2002), but the same group later reported retrieval speeds of about 1-2 seconds, decreasing the apparent advantage over clathrin-mediated endocytosis (Wu et al., 2005).

A different approach to investigate fusion events involves the stimulation of nerve terminals in presence of lipophilic styryl FM dye (Betz and Bewick, 1992) that inserts into the lumenal leaflet of fused vesicle membranes and thus labels the vesicles upon retrieval into the terminal; exocytosis can then be monitored as the decrease in fluorescence (release of dye) during subsequent stimulation. As kiss-and-run, by 
definition, only connects to the plasma membrane by the opening of a (arguably) small and transient fusion pore, more hydrophilic dyes should be released faster than more lipophilic ones. This observation indeed served as an argument for kiss-and-run (Klingauf et al., 1998), but subsequent studies failed to confirm this finding, as also a more hydrophobic dye was readily released during exocytosis (Fernandez-Alfonso and Ryan, 2004). In a different attempt to differentiate between full-collapse fusion and kissand-run, FM dye experiments were combined with the application of the hydrophilic quencher bromophenol blue, according to the following rationale: if a labeled vesicle underwent full-collapse fusion, it would release all of its FM dye, whereas the transient fusion pore of a kiss-and-run event would only allow partial release of FM dye, so that the remaining dye molecules would be subject to quenching by bromophenol blue (Harata et al., 2006). However, a serious pitfall with this approach is the fact that the dye released by full-collapse fusion events could remain bound to the plasma membrane in the tight space of the synaptic cleft; as the dye would be equally subject to bromophenol blue quenching here, the fusion event would be falsely interpreted as kiss-and-run (see discussion in Granseth et al., 2006).

Yet another innovative tool to study synaptic vesicle exocytosis are chimeras of a $\mathrm{pH}$-dependent green fluorescent protein (GFP) variant (pHluorin) linked to the lumenal terminus of a synaptic vesicle protein (Miesenbock et al., 1998). While the pHluorin moiety is brightly fluorescent at neutral extracellular $\mathrm{pH}$ (to which fused vesicles are exposed), it is quenched at the acidic $\mathrm{pH}$ of internal synaptic vesicles, rendering the pHluorin fluorescence an indicator of vesicle fusion. Using this tool, the time course of pHluorin quenching suggested the occurrence of fast cycling events with fusion pore durations of about 0.5-1 second (Gandhi and Stevens, 2003), but a subsequent study relying on similar experiments could not detect any such fast events (Granseth et al., 2006).

In a recent study, Richard Tsien and coworkers (Zhang et al., 2009) used semiconductor Quantum Dots (Qdots) to label recycling synaptic vesicles. By taking advantage of the pH-dependence of the Qdots (which remains a matter of debate, see Pons and Mattoussi, 2009), Tsien and coworkers interpreted brief "upticks" in fluorescence as the brief opening and closing of a Qdot-labeled synaptic vesicle undergoing kiss-and-run fusion. Full-collapse fusion however was characterized by prolonged elevated fluorescence, which could either return to baseline by endocytosis (and re-acidification of the vesicle) or be lost completely if the Qdot escaped into the synaptic cleft and diffused away. As Lagnado and coworkers noted (Granseth et al., 2009), fast retrieval kinetics could also be attributed to a steric effect of the Qdots: a 
synaptic cleft in this preparation measures about $20 \mathrm{~nm}$ across and is tightly packed with extracellular matrix and cell adhesion proteins; the Qdots used by Tsien and coworkers are also about $20 \mathrm{~nm}$ in diameter and will thus not be very quick in diffusing away from a fused vesicle (Smith et al., 2010). It could be conceived that the Qdot jammed in-between vesicle and plasma membrane prevents the fused vesicle from fully collapsing into the plasma membrane and somehow promotes retrieval instead. It is also possible that an escaped Qdot could be taken up by an adjacent readily retrievable vesicle (as suggested by Wienisch and Klingauf, 2006), which is however not expected to account alone for the observed brief upticks.

In addition to these controversies regarding kiss-and-run, a major shortcoming of the model relates to the burden of maintaining the composition of synaptic vesicles even after multiple rounds of fusion. As no molecular players of kiss-and-run have been described, one has to postulate that kiss-and-run fusion does not involve diffusion of vesicle proteins and lipids into the plasma membrane and vice versa. From our current knowledge, however, this claim can only be regarded as hypothetical.

In conclusion, the kiss-and-run model remains what the name suggests - merely a model. The controversies regarding the reports on kiss-and-run are too substantial and too manifold to draw solid conclusions with respect to the relevance of this recycling mode. For the scope of this study, it is especially important to note that endosomal recycling should not a priori be rejected based on the existence of a potentially better-suited recycling mechanism. Both kiss-and-run and endosomal recycling come with their intrinsic advantages and disadvantages that will need to be elucidated in order to better understand the complex issue of synaptic vesicle recycling. 


\subsection{Aim of the Project}

As becomes evident from the considerations above, the field of synaptic vesicle recycling is still awaiting conclusive evidence for both the kiss-and-run and the endosomal recycling models. Although both models have received (at least at some point) their share of attention, none of them remained free of controversy.

As no mechanistic and molecular data is currently available for the kiss-and-run model, technical limitations (time resolution, diffraction limit etc.) and our lack of knowledge would require large screening efforts to first identify possible molecules unique to kiss-and-run fusion that, if successful, could afterwards be characterized in more detail to study the mechanisms governing kiss-and-run. Failure to identify any molecular players or mechanistic aspects of kiss-and-run (which is not impossible in view of the contradictory reports), would, however, not be accepted as valid proof against kiss-and-run.

The situation is quite different for the endosomal recycling model. Taken the molecular and functional knowledge about endosomes and given the technological possibilities of today, we should be able to devise experiments to test successfully the function of endosomes in synapses. The existence of several targets for the specific labeling and perturbation of the early endosome will enable us to use a multitude of imaging approaches to visualize the interplay between synaptic vesicles and early endosomes.

Therefore, we set out to verify whether endosomes are indeed involved in the recycling of synaptic vesicles in small synapses of the central nervous system, focusing on cultured hippocampal neurons as the model system of choice. If successful, we would attempt to investigate the relevance of endosomal recycling for the different vesicle pools by employing conventional and advanced fluorescence imaging techniques in combination with photo-oxidation electron microscopy. An additional point of investigation was to determine how tightly (and possibly at which recycling steps) the molecular identity of synaptic vesicles is controlled and maintained.

Overall, this study clearly offers the potential to provide new impetus to the synaptic vesicle recycling field: we are now in the position to tackle the long-standing question of endosomal involvement in what is probably the most elementary process in the pre-synapse. Furthermore, the results from this study, although focusing on the investigation of the endosomal pathway, could add new insights about general principles in synaptic vesicle recycling that might also prove useful in interpreting data relating to the kiss-and-run model. 


\section{Materials AND MethodS}

\subsection{Antibodies}

Primary antibodies were used as available from Synaptic Systems GmbH, Göttingen (SySy; www.sysy.com), Abcam plc, Cambridge, UK (Abcam; www.abcam.com), Becton Dickinson GmbH, Heidelberg (BD; www.bd.com/de), Affinity BioReagents / Thermo Fisher Scientific Inc., Rockford, Il, USA (ABR; www.bioreagents.com), Sigma-Aldrich Chemie GmbH, Steinheim (Sigma; www.sigmaaldrich.com/germany) and Echelon Biosciences Incorporated, Salt Lake City, UT, USA (Echelon; www.echelon-inc.com). All primary antibodies used in this study are listed in Table 2-1.

All secondary antibodies were obtained from Dianova GmbH, Hamburg (www.dianova.de) and were either purchased as fluorescent conjugates (labeled with Cy2, Сy3 or Cy5) or were conjugated to the dye Atto 532, Atto 590 or Atto 647N according to the supplier's protocol (Atto-Tec GmbH, Siegen; www.atto-tec.com).

Table 2-1: Primary antibodies used throughout the study

\begin{tabular}{|c|c|c|c|}
\hline Antibody target & Antibody type & Notes & Reference \\
\hline $\begin{array}{l}\text { ADP Ribosylation } \\
\text { Factor }\end{array}$ & $\begin{array}{l}\text { mouse monoclonal; } \\
\text { clone 1D9 }\end{array}$ & Also known as Arf & Abcam \\
\hline Amphiphysin & rabbit polyclonal & & SySy \\
\hline $\mathrm{AP}-3 \beta-2$ & chicken polyclonal & & Abcam \\
\hline $\begin{array}{l}\text { AP1 complex } \\
\text { subunit } \gamma-1\end{array}$ & rabbit polyclonal & & $\mathrm{ABR}$ \\
\hline AP180 & rabbit polyclonal & & SySy \\
\hline $\begin{array}{l}\text { Clathrin heavy } \\
\text { chain }\end{array}$ & $\begin{array}{l}\text { mouse monoclonal; } \\
\text { clone } 23\end{array}$ & & $\mathrm{BD}$ \\
\hline Dynamin $1,2,3$ & rabbit polyclonal & & SySy \\
\hline Endophilin & rabbit polyclonal & & SySy \\
\hline GABA-transporter & $\begin{array}{l}\text { rabbit polyclonal; } \\
\text { R22 }\end{array}$ & & $\begin{array}{l}\text { Kind gift from Reinhard } \\
\text { Jahn (Max-Planck Institute } \\
\text { for Biophysical Chemistry, } \\
\text { Göttingen) }\end{array}$ \\
\hline GFP & $\begin{array}{l}\text { rabbit polyclonal; } \\
\text { ab290 }\end{array}$ & & Abcam \\
\hline HA-tag & mouse monoclonal & & $\begin{array}{l}\text { Kind gift from Reinhard } \\
\text { Jahn }\end{array}$ \\
\hline Munc18-1 & $\begin{array}{l}\text { mouse monoclonal; } \\
\text { clone } 131.1\end{array}$ & & SySy \\
\hline NMDA-receptor & $\begin{array}{l}\text { mouse monoclonal; } \\
\text { clone } 54.1\end{array}$ & $\begin{array}{l}\text { Used without fixation, for } \\
\text { optimal epitope recognition }\end{array}$ & SySy \\
\hline
\end{tabular}




\begin{tabular}{|c|c|c|c|}
\hline NSF & $\begin{array}{l}\text { mouse monoclonal; } \\
\text { clone } 83.11\end{array}$ & & SySy \\
\hline PI(3)P & $\begin{array}{l}\text { mouse monoclonal; } \\
\text { Z-P003 }\end{array}$ & & Echelon \\
\hline Rab4 & $\begin{array}{l}\text { mouse monoclonal; } \\
\text { clone } 7\end{array}$ & & $\mathrm{BD}$ \\
\hline Rab5 & $\begin{array}{l}\text { mouse monoclonal; } \\
\text { clone } 621.1\end{array}$ & $\begin{array}{l}\text { Used without fixation, for } \\
\text { optimal epitope recognition }\end{array}$ & $\begin{array}{l}\text { (Fischer von Mollard et al., } \\
\text { 1994) }\end{array}$ \\
\hline SNAP-25 & $\begin{array}{l}\text { mouse monoclonal; } \\
\text { clone } 71.1\end{array}$ & $\begin{array}{l}\text { Plasma membrane exocytic Q- } \\
\text { SNARE }\end{array}$ & SySy \\
\hline SNAP-25 & rabbit polyclonal & $\begin{array}{l}\text { Plasma membrane exocytic Q- } \\
\text { SNARE }\end{array}$ & (Aguado et al., 1996) \\
\hline Synaptobrevin 2 & $\begin{array}{l}\text { mouse monoclonal; } \\
\text { clone } 69.1\end{array}$ & $\begin{array}{l}\text { Synaptic vesicle exocytic R- } \\
\text { SNARE; Also known as Vamp2 }\end{array}$ & SySy \\
\hline Synaptojanin 1 & rabbit polyclonal & $\begin{array}{l}\text { Against C-terminus; affinity- } \\
\text { purified from serum }\end{array}$ & SySy \\
\hline Synaptophysin & $\begin{array}{l}\text { mouse monoclonal; } \\
\text { clone } 7.2\end{array}$ & & SySy \\
\hline Synaptophysin & $\begin{array}{l}\text { rabbit polyclonal; } \\
\text { G96 }\end{array}$ & & (Jahn et al., 1985) \\
\hline Synaptotagmin I & $\begin{array}{l}\text { mouse monoclonal; } \\
\text { clone } 41.1\end{array}$ & & SySy \\
\hline Synaptotagmin I & $\begin{array}{l}\text { mouse monoclonal; } \\
\text { clone } 604.2\end{array}$ & $\begin{array}{l}\text { Against luminal (extracellular) } \\
\text { domain; We also obtained this } \\
\text { antibody in its biotinylated form } \\
\text { or directly labeled to the } \\
\text { fluorescent dye Oyster550 or } \\
\text { Atto } 647 \mathrm{~N}\end{array}$ & SySy; (Willig et al., 2006) \\
\hline Syntaxin 1 & $\begin{array}{l}\text { mouse monoclonal; } \\
\text { clone } 78.2\end{array}$ & $\begin{array}{l}\text { Plasma membrane exocytic Q- } \\
\text { SNARE }\end{array}$ & SySy \\
\hline Syntaxin 1 & $\begin{array}{l}\text { mouse monoclonal; } \\
\text { HPC1 }\end{array}$ & $\begin{array}{l}\text { Plasma membrane exocytic Q- } \\
\text { SNARE }\end{array}$ & (Barnstable et al., 1985) \\
\hline Syntaxin 13 & rabbit polyclonal & & (Rizzoli et al., 2006) \\
\hline Syntaxin 6 & $\begin{array}{l}\text { mouse monoclonal; } \\
\text { clone } 30\end{array}$ & & $\mathrm{BD}$ \\
\hline Uncoating ATPase & $\begin{array}{l}\text { mouse monoclonal; } \\
\text { clone 3C5 }\end{array}$ & Also known as Hsc70 & SySy \\
\hline Vglut1 & $\begin{array}{l}\text { rabbit polyclonal; } \\
\text { shigeo } 2\end{array}$ & & $\begin{array}{l}\text { Kind gift from Reinhard } \\
\text { Jahn }\end{array}$ \\
\hline $\begin{array}{l}\text { Voltage-gated } \mathrm{Ca}^{2+} \\
\text { channel }\end{array}$ & rabbit polyclonal & Affinity-purified from serum & SySy \\
\hline Vti1a & $\begin{array}{l}\text { mouse monoclonal; } \\
\text { clone } 103.3\end{array}$ & & (Rizzoli et al., 2006) \\
\hline$\alpha$-SNAP & $\begin{array}{l}\text { mouse monoclonal; } \\
\text { clone } 77.1\end{array}$ & & SySy \\
\hline$\beta$-actin & $\begin{array}{l}\text { mouse monoclonal; } \\
\text { clone AC-15 }\end{array}$ & & Sigma \\
\hline
\end{tabular}




\subsection{Chemicals and Reagents}

All standard chemicals were purchased from Sigma or Merck KGaA, Darmstadt (www.merck.de). Streptavidin-coated Qdots (525, 565, 605 and 655) and multicolor TetraSpeck $^{\mathrm{TM}}$ beads (diameter of 100 or $200 \mathrm{~nm}$ ) were purchased from Invitrogen GmbH, Darmstadt (www.invitrogen.com). Dynasore was purchased from ChemBridge Corporation, San Diego, CA, USA (www.chembride.com) and used at a concentration of $80 \mu$ M. Methyl- $\beta$-cyclodextrin was purchased from Sigma. The buffers and solutions used throughout the study are listed in Table 2-2.

\section{Table 2-2: Buffers and solutions}

\begin{tabular}{|c|c|}
\hline Solution & Ingredients \\
\hline Homogenization buffer & $250 \mathrm{mM}$ sucrose, $3 \mathrm{mM}$ imidazole, $\mathrm{pH} 7.3$ \\
\hline Mowiol & 6 g glycerol AR, 2.4 g Mowiol $^{\circledR} 4-88$ (Merck), 6 ml H${ }_{2} \mathrm{O}, 12 \mathrm{ml} 0.2 \mathrm{M}$ Tris, pH 7.2 \\
\hline Normal Tyrode & $\begin{array}{l}124 \mathrm{mM} \mathrm{NaCl}, 5 \mathrm{mM} \mathrm{KCl}, 2 \mathrm{mM} \mathrm{CaCl}, 1 \mathrm{mM} \mathrm{MgCl} 2,30 \mathrm{mM} \text { glucose and } 25 \mathrm{mM} \\
\text { HEPES (4-(2-hydroxyethyl)-1-piperazineethanesulfonic acid), pH } 7.4\end{array}$ \\
\hline $\begin{array}{l}\text { Phosphate buffered saline } \\
\text { (PBS) }\end{array}$ & $150 \mathrm{mM} \mathrm{NaCl}, 20 \mathrm{mM} \mathrm{Na}_{2} \mathrm{HPO}_{4}, \mathrm{pH} 7.4$ \\
\hline PC12 saline & $\begin{array}{l}130 \mathrm{mM} \mathrm{NaCl}, 4 \mathrm{mM} \mathrm{KCl}, 48 \mathrm{mM} \text { glucose, } 10 \mathrm{mM} \text { HEPES, } 5 \mathrm{mM} \mathrm{CaCl} 2,1 \mathrm{mM} \\
\mathrm{MgCl} 2, \mathrm{pH} 7.3\end{array}$ \\
\hline Sodium buffer & $\begin{array}{l}10 \mathrm{mM}_{\text {glucose, }} 5 \mathrm{mM} \mathrm{KCl}, 140 \mathrm{mM} \mathrm{NaCl}, 5 \mathrm{mM} \mathrm{NaHCO}_{3}, 1 \mathrm{mM} \mathrm{MgCl}_{2}, 1.2 \mathrm{mM} \\
\mathrm{Na}_{2} \mathrm{HPO}_{4}, 20 \mathrm{mM} \text { HEPES, pH } 7.4\end{array}$ \\
\hline Sucrose buffer & $320 \mathrm{mM}$ sucrose, $5 \mathrm{mM}$ HEPES, pH 7.4 \\
\hline
\end{tabular}

Plasmid DNA for transfections was prepared either with the EndoFree Plasmid Maxi Kit from Qiagen GmbH, Hilden (www.qiagen.com) or with the NucleoBond PC 500 maxi kit from Machery-Nagel GmbH \& Co. KG, Düren (www.mn-net.com), according to the supplier's protocol.

Rab5-GFP constructs (wild-type Rab5 or the dominant active (Q79L) mutant) were kindly provided by Mikael Simons (Max-Planck Institute of Experimental Medicine, Göttingen). The constructs have been previously tested in PC12 cell culture and were found to be functional (Bethani et al., 2007).

The constructs for expression of the dominant-negative soluble Syntaxin 13 fragment were designed by cloning the cytosolic domain of Syntaxin 13 (aa 1-250) or the cytosolic domain lacking the SNARE domain (aa 1-186) into the pIRES2-AcGFP1 vector (Clontech Laboratories, Inc., Mountain View, CA, USA; www.clontech.com). Via its internal ribosomal entry site (IRES), this construct drives the expression of GFP and soluble Syntaxin 13 fragment from one mRNA. As the Syntaxin 13 fragment is not fused 
to the GFP, this approach avoids any tagging artifacts, while transfected cells can still be readily identified by the simultaneously expressed GFP. The expression of Syntaxin 13 was confirmed by performing immunostainings in PC12 cells, showing that all GFP positive cells also expressed cytosolic Syntaxin 13. Cloning of soluble Syntaxin 13 fragments was performed with the help of I. Bethani and F. Opazo (STED Microscopy of Synaptic Function, European Neuroscience Institute Göttingen).

\subsection{Microscopy}

\subsubsection{Epi-fluorescence microscopy}

All filters were purchased from Chroma Technology Corporation, Bellow Falls, VT, USA (www.chroma.com) and are listed in Table 2-3.

Table 2-3: Filter-sets used for epi-fluorescence microscopy

\begin{tabular}{l|l|l|l} 
Name of filter & Exciter & Beamsplitter & Emitter \\
\hline DAPI & $350 / 50 \mathrm{D}$ & $400 \mathrm{DCLP}$ & $460 / 50 \mathrm{D}$ \\
\hline Alexa 488 & $480 / 40 \mathrm{HQ}$ & $505 \mathrm{LP} \mathrm{Q}$ & $527 / 30 \mathrm{HQ}$ \\
\hline TRITC & $545 / 30 \mathrm{HQ}$ & $570 \mathrm{LP} \mathrm{Q}$ & $610 / 75 \mathrm{HQ}$ \\
\hline Cy5 & $620 / 60 \mathrm{HQ}$ & $660 \mathrm{LP} \mathrm{Q}$ & $700 / 75 \mathrm{HQ}$
\end{tabular}

\subsubsection{Olympus setup}

An inverted Olympus IX71 microscope (Olympus Europa Holding GmbH, Hamburg; www.olympus.de/microscopy), equipped with an F-View II CCD camera (12 bit; $1376 \times$ 1032 pixels; $6.45 \mu \mathrm{m}$ pixel size), was used for most standard applications. All objectives used with this setup were from Olympus and are listed in Table 2-4.

Table 2-4: Olympus objective specifications

\begin{tabular}{l|l|l|l} 
Magnification & Numerical Aperture & Immersion type & Lens type \\
\hline $100 \mathrm{x}$ & 1.45 & Oil & PlanApo TIRF \\
\hline $100 \mathrm{x}$ & 1.4 & Oil & UPlanSApo \\
\hline $60 \mathrm{x}$ & 1.35 & Oil & UPlanSApo \\
\hline $40 \mathrm{x}$ & 0.75 & Dry & UPlanFL N \\
\hline $20 \mathrm{x}$ & 0.5 & Dry & UPlanFL N
\end{tabular}




\subsubsection{Zeiss Axiovert setup}

A Zeiss Axiovert 200M microscope (Carl Zeiss MicroImaging GmbH, Jena; www.zeiss.de/mikro), equipped with a 100x, 1.4 NA oil immersion objective and a Princeton Instruments CCD camera (Princeton Instruments, Trenton, NJ, USA; www.princetoninstruments.com) with a Kodak chip (Eastman Kodak Company, Rochester, NY, USA; www.kodak.com; 12 bit; $1317 \times 1035$ pixels; $6.8 \mu$ m pixel size), was used for the optimizations of the assay using isolated synaptic vesicles on glass coverslips (see 2.12) and for some of the final experiments using this assay. Alternatively, the Olympus setup (above) was used for this assay. The results from both setups were pooled because the comparison of control stainings revealed no detectable difference between the datasets.

\subsubsection{Zeiss Examiner setup}

For accurate detection of low signals, an upright Zeiss Examiner.Z1 microscope was equipped with the Olympus 100x, 1.45 NA TIRF objective (see Table 2-4) and with a high-sensitivity QuantEM:512SC EM-CCD camera (Photometrics, Tuscon, AZ, USA; www.photomet.com; 16 bit; $512 \times 512$ pixels; $16 \mu \mathrm{m}$ pixel size).

\subsubsection{Zeiss Axioskop setup}

Photo-oxidation illumination and monitoring was performed with an upright Zeiss Axioskop 2 microscope, using the Olympus 20x, 0.5 NA objective (see Table 2-4). Images were acquired with a Zeiss AxioCam Mrm CCD camera (12 bit; $1388 \times 1040$ pixels; 6.45 $\mu \mathrm{m}$ pixel size).

Table 2-5: Laser lines for confocal laser scanning microscopy

\begin{tabular}{l|l} 
Laser & Excitation lines \\
\hline Argon $(100 \mathrm{~mW})$ & $458 \mathrm{~nm}, 476 \mathrm{~nm}, 488 \mathrm{~nm}, 496 \mathrm{~nm}, 514 \mathrm{~nm}$ \\
\hline HeliumNeon $(1 \mathrm{~mW})$ & $543 \mathrm{~nm}$ \\
\hline HeliumNeon $(2 \mathrm{~mW})$ & $594 \mathrm{~nm}$ \\
\hline HeliumNeon $(10 \mathrm{~mW})$ & $633 \mathrm{~nm}$
\end{tabular}




\subsubsection{Confocal and stimulated emission depletion microscopy}

\subsubsection{Standard Leica setup}

Standard confocal and single color stimulated emission depletion (STED) imaging were performed using a Leica TCS STED microscope (Leica Microsystems GmbH, Wetzlar; www.leica-microsystems.com), equipped with a Leica 100x, 1.4 NA STED oil immersion objective. The appropriate excitation laser lines (see Table 2-5) are combined by an acousto-optical tunable filter (AOTF). The STED dye (Atto 647N) was excited with a 635 $\mathrm{nm}$ diode laser and depletion was stimulated at $750 \mathrm{~nm}$, using a Spectra-Physics MaiTai tunable laser (Newport Spectra-Physics GmbH, Darmstadt; www.newport.com). Appropriate detection bands were set via acousto-optical beam splitters (AOBS). Standard confocal signals were detected using photomultiplier tubes (PMT); STED signals were detected using the more sensitive avalanche photodiode (APD).

\subsubsection{Live-STED setup}

Video-rate (28 frames per second) live-STED imaging was performed largely as described (Westphal et al., 2008) with the help of V. Westphal (NanoBiophotonics, MPI for Biophysical Chemistry, Göttingen). A second pulsed laser diode (picoTA 490; TOPTICA Photonics AG, Gräfelfing; www.toptica.com) was added for the excitation at $490 \mathrm{~nm}$ (for the GFP). The laser was coupled into a polarization-maintaining single mode fiber and the collimated output was collinearly combined with the $637 \mathrm{~nm}$ excitation (for Atto 647N) using a dichroic mirror. A second detection channel (for the GFP) was implemented by splitting the emitted light after the confocal pinhole with a Semrock FF458 dichroic mirror featuring a second edge at $609 \mathrm{~nm}$ (Semrock, Rochester, NY, USA; www.semrock.com). The lower wavelength portion was directed through an emission filter (HQ525/60m) onto a second avalanche photodiode (SPCM-AQR13; PerkinElmer Inc., Waltham, MA, USA; www.perkinelmer.com).

\subsubsection{2-color STED setup}

2-color STED microscopy was performed with a similar setup as described previously (Wildanger et al., 2008). Imaging was performed with the help of A. Punge and J. Bückers (NanoBiophotonics, MPI for Biophysical Chemistry, Göttingen). Two arrangements of excitation and depletion (STED) laser beams were combined, all beams being provided by a single super continuum laser source (SC-450 HP, Fianium Ltd, Southampton, UK; www.fianium.com). The excitation wavelengths were set to $570 \pm$ $5 \mathrm{~nm}$ (for Atto 590) and $650 \pm 5 \mathrm{~nm}$ (for Atto 647N). The depletion wavelengths were set to $720 \pm 10 \mathrm{~nm}$ (Atto 590) and $755 \pm 15 \mathrm{~nm}$ (Atto 647N). The fluorescence of the 
dyes was detected in the spectral ranges of $600-640 \mathrm{~nm}$ for Atto 590 and $660-690 \mathrm{~nm}$ for Atto 647N, respectively. Imaging was performed using a Leica 100x, 1.4 NA oil objective (PL APO 100x/1.40-0.7 Oil). Crosstalk between the two detection channels was eliminated by means of linear unmixing.

\subsubsection{Electron microscopy}

Transmission electron micrographs were acquired using a Zeiss EM 902A electron microscope, equipped with a Proscan CCD camera (Proscan CCD HSS 512/1024; Proscan elektronische Systeme, Lagerlechfeld; www.proscan.de; 8 bit; $1024 \times 1024$ pixels).

\subsection{Neuronal Cell Culture}

Primary cultures from P1 rat Hippocampus were prepared as described (Willig et al., 2006). Briefly, hippocampi were dissected and dissociated. Neurons were plated on an astrocyte feeding layer (which had been grown for one week) and the medium was replaced two days after plating.

\subsection{Preparation of Lumenally Labeled Synaptic Vesicles}

To compare the protein composition of actively recycling synaptic vesicles and the total pool of vesicles in an in vitro assay, the actively cycling vesicles had to be specifically labeled. To this end, synaptic vesicle enriched LS1-fractions were prepared according to a modified protocol from De Camilli and coworkers (Huttner et al., 1983; see below).

\subsubsection{Preparation of active synaptosomes}

Two rat brains were collected into $30 \mathrm{ml}$ ice-cold sucrose buffer ( $320 \mathrm{mM}$ sucrose, $5 \mathrm{mM}$ HEPES, pH 7.4). Unless otherwise noted, the preparation was kept on ice at all times and all centrifugations were performed at $4^{\circ} \mathrm{C}$. Homogenization with a $50 \mathrm{ml}$ Teflon-glass homogenizer (10 strokes at $900 \mathrm{rpm}$ ) was followed by centrifugation at $5000 \mathrm{rpm}$ for 2 minutes in an SS34 rotor (Thermo Fisher Scientific Inc., Waltham, MA, USA; www.thermofisher.com) to pellet large cellular fragments, blood cells and nuclei (fraction P1). Re-centrifugation of the supernatant (fraction S1) at 11,000 rpm for 12 minutes resulted in a pellet mainly consisting of synaptosomes, mitochondria and myelin (fraction P2). The supernatant was discarded and the pellet (except for the brown mitochondrial component) was re-suspended in $4 \mathrm{ml}$ sucrose buffer. The solution was loaded onto two Ficoll step-gradients (from top to bottom: $4 \mathrm{ml} 6 \%, 1 \mathrm{ml}$ 
9\% and $4 \mathrm{ml} \mathrm{13 \%} \mathrm{Ficoll} \mathrm{in} \mathrm{sucrose} \mathrm{buffer)} \mathrm{and} \mathrm{centrifuged} \mathrm{at} \mathrm{22,500} \mathrm{rpm} \mathrm{for} 35$ minutes in an SW41 Ti rotor (Beckman Coulter Inc., Brea, CA, USA; www.beckmancoulter.com). The bands at the $6 \% / 9 \%$-interface and at the $9 \% / 13 \%$-interface from both gradients were combined and diluted in $20 \mathrm{ml}$ sucrose buffer. Re-centrifugation to remove residual Ficoll was performed at 11,000 rpm for 12 minutes in an SS34 rotor, yielding a pellet containing relatively pure and active synaptosomes (fraction P2').

\subsubsection{Labeling of actively recycling synaptic vesicles}

The pellet containing active synaptosomes (fraction P2') was re-suspended in $20 \mathrm{ml}$ sodium buffer ( $10 \mathrm{mM}$ glucose, $5 \mathrm{mM} \mathrm{KCl}, 140 \mathrm{mM} \mathrm{NaCl}, 5 \mathrm{mM} \mathrm{NaHCO}{ }_{3}, 1 \mathrm{mM} \mathrm{MgCl}_{2}, 1.2$ $\mathrm{mM} \mathrm{Na}_{2} \mathrm{HPO}_{4}, 20 \mathrm{mM}$ HEPES, pH 7.4) and centrifuged at 6,000 rpm for 6 minutes in an SS34 rotor. The resulting pellet was gently re-suspended in $6 \mathrm{ml}$ sodium buffer and warmed up to $37^{\circ} \mathrm{C}$ in a water bath. To avoid sedimentation of the synaptosomes, the sample was shaken at $135 \mathrm{rpm}$ while incubating for 15-20 minutes. Subsequently, the synaptosomes were equilibrated with $1.3 \mathrm{mM} \mathrm{CaCl}_{2}$ and the fluorescent probe to be endocytosed. The final concentrations of the probes were $400 \mu \mathrm{M}$ for FM 2-10 (Invitrogen) and $15 \mu \mathrm{g} / \mathrm{ml}$ for the anti-Synaptotagmin 604.2-0yster550 antibody (syt $t_{\text {lum, }}$ see Table 2-1). Stimulation of the synaptosomes was achieved by adding $50 \mathrm{mM} \mathrm{KCl}$ and incubating for 5 minutes at $37^{\circ} \mathrm{C}$. The synaptosomes were returned to a resting state by adding $20 \mathrm{ml}$ ice-cold sodium buffer and pelleting them at 6,000 rpm for 4-5 minutes in an SS34 rotor. Non-internalized dye was washed away by re-suspending in 10-20 ml icecold sodium buffer and pelleting at 6,000 rpm for 4-5 minutes in an SS34 rotor. This washing procedure was repeated three times before the synaptosomes were finally repelleted. After removing the buffer, the pellet was gently rinsed with $\mathrm{ddH}_{2} \mathrm{O}$ (double distilled water) to eliminate all buffer traces. At this stage, the synaptosomal preparation contains actively recycling (recently endocytosed) synaptic vesicles that are lumenally (internally) labeled with FM 2-10 or 604.2-Oyster550.

\subsubsection{Isolation of LS1-fraction synaptic vesicles}

Labeled synaptosomes (fraction P2') were broken by hypo-osmotic lysis as follows. The soft pellet from the synaptosome preparation was homogenized by 5-10 strokes at 2,000 rpm after transferring it into a $5 \mathrm{ml}$ homogenizer and adding 6-9 volumes of $\mathrm{ddH}_{2} \mathrm{O}$. Protease inhibitors $(1 \mu \mathrm{g} / \mathrm{ml}$ Pepstatin A, $1 \mathrm{mM}$ phenyl-methane-sulphonylfluoride [PMSF]) were added immediately after lysis. The liberated organelles were quickly suspended by adding $1 / 4$ of the total lysate's volume of a $5 \mathrm{x}$ homogenization buffer stock (resulting concentration: $250 \mathrm{mM}$ sucrose, $3 \mathrm{mM}$ imidazole, $\mathrm{pH}$ 7.3). To remove large membranes and large organelles, a final centrifugation step at 25,000 rpm 
was performed for 20-30 minutes in a TLA-100.3 rotor (Beckman Coulter). The supernatant (fraction LS1) was collected, snap-frozen in liquid nitrogen and stored at $-80^{\circ} \mathrm{C}$ until use.

\subsubsection{Determination of protein concentration}

The protein concentrations of the LS1-fractions were determined by means of Bradford assay (Bradford, 1976). Briefly, the Coomassie G-250 dye binds to protein, resulting in a red-shifted absorption maximum of the dye (from $465 \mathrm{~nm}$ to $595 \mathrm{~nm}$ ). As a measure of total protein concentration, the absorption at $595 \mathrm{~nm}$ is monitored photometrically. The protein concentration is calculated from a standard curve, measured from solutions of known protein concentration (bovine serum albumin fraction V (BSA); AppliChem GmbH, Darmstadt; www.applichem.de).

\subsection{PHluorin Expression and Imaging}

\subsubsection{PHluorin constructs}

Superecliptic pHluorin (Miesenbock et al., 1998) was obtained with the agreement of the Sloan-Kettering Institute for Cancer Research, NY, USA, and Leon Lagnado (MRC Laboratory of Molecular Biology, Cambridge, UK) kindly provided SynaptopHluorin (Synaptobrevin fused to pHluorin). Phluorin constructs were prepared with the help of S. V. Barysch (STED Microscopy of Synaptic Function, European Neuroscience Institute Göttingen).

Table 2-6: Primers for SNARE Amplification

\begin{tabular}{l|l|l}
\multicolumn{1}{c|}{ SNARE } & Primer 1 & Primer 2 \\
\hline Syntaxin 13 & $\begin{array}{l}\text { CCGCTCGA-GCACCATG-TCCTACGG- } \\
\text { TCCCTTAG }\end{array}$ & $\begin{array}{l}\text { ACGTCACC-GGTTCTTA-GAAGCAAC- } \\
\text { CCAGATAA-C }\end{array}$ \\
\hline Vti1a & $\begin{array}{l}\text { ATCCGCTC-GAGCACCA-TGTCAGCC- } \\
\text { GACTTCGA-AGG }\end{array}$ & $\begin{array}{l}\text { TATCGGGA-TCCCGGTG-TCCTCTGA- } \\
\text { CAAAAAAA-GTG }\end{array}$ \\
\hline Syntaxin 6 & $\begin{array}{l}\text { ATCCGCTC-GAGCACCA-TGTCCATG- } \\
\text { GAGGACCC-CTTC }\end{array}$ & $\begin{array}{l}\text { TATCGGGA-TCCCGCAG-CACTAAGA- } \\
\text { AGAGGATG-AGC }\end{array}$ \\
\hline Syntaxin 1 & $\begin{array}{l}\text { ATCCGCTC-GAGCACCA-TGAAGGAC- } \\
\text { CGAACCCA-GG }\end{array}$ & $\begin{array}{l}\text { ATACGTCA-CCGGTTTC-CAAAGATG- } \\
\text { CCCCCGAT-GG }\end{array}$
\end{tabular}


For the generation of the other SNARE-pHluorins (Syntaxin 13, Syntaxin 6, Vti1a, Syntaxin 1), a pHluorin entry vector was constructed on the basis of the pEGFP-N1 vector (Clontech). PHluorin, containing an $\mathrm{N}$-terminal linker sequence (AGCGGCGGAAGCGGCG-GGACCGGT-GGA), was amplified by polymerase chain reaction (PCR) from the SynaptopHluorin construct using the primers CGGGATCC-AAGCGGCG-GAAGCGGCGGGA and ATAGTTTA-GCGGCCGC-TCATGCCA-TGTGTAT-CCCAGC. The enhanced-GFP was then replaced by the resulting PCR fragment after BamHI and NotI restriction. The SNAREs were amplified by PCR using the primers shown in Table 2-6. Syntaxin 1 and Syntaxin 13 were cloned into the pHluorin entry vector via XhoI and AgeI/XmaI (SNARE PCR fragment digested with XhoI and AgeI, vector digested with XhoI and XmaI), Syntaxin 6 and Vti1a via XhoI and BamHI. PCR was performed using the New England Biolabs Phusion ${ }^{\circledR}$ high fidelity kit (New England Biolabs Inc., Ipswich, MA, USA; www.neb.com). T4 ligase and restriction enzymes were purchased from New England Biolabs. Constructs were sequenced by Eurofins MWG GmbH, Ebersberg (www.eurofinsdna.com). All constructs were generated from rat (rattus norvegicus) sequences.

\subsubsection{PHluorin expression}

Hippocampal cultures were transfected between 7 and 11 days in vitro (DIV) with the pHluorin constructs by magnetofection. NeuroMag para-magnetic beads (OZ biosciences, Marseille, France; www.ozbiosciences.com) were used according to the supplier's protocol. In brief, $2 \mu \mathrm{g}$ DNA were diluted in $100 \mu \mathrm{l}$ Neurobasal-A medium (Invitrogen; supplemented with $10 \mathrm{mM} \mathrm{MgCl}_{2}$ and $5 \mathrm{mM} \mathrm{HEPES}$, adjusted to $\mathrm{pH} 7.5$ ) and added to $7 \mu \mathrm{l}$ NeuroMag beads. DNA-bead-complexes were allowed to form for 15-20 minutes at room temperature, before adding drop-by-drop onto neurons grown on an $18 \mathrm{~mm}$ coverslip. Finally, the culture plate was placed on a super magnetic plate $\mathrm{COZ}$ Biosciences) in the culture incubator for 15 minutes to precipitate the DNA-beadcomplexes on the cells.

\subsubsection{PHluorin imaging and analysis}

PHluorin imaging was generally performed 24 hours after transfection. Cells were incubated for 10 minutes with anti-Synaptotagmin antibody (against the lumenal domain; clone 604.2; see Table 2-1 above) directly labeled with the fluorescent dye Oyster550 and then briefly washed twice. This procedure labels actively exo/endocytosing synaptic vesicles and thus active synaptic boutons (Willig et al., 2006). Cultures were stimulated using a custom-made platinum plate electrode $(8 \mathrm{~mm}$ distance between the 2 electrodes) by delivering 100-milliampere shocks at $20 \mathrm{~Hz}$, using an A385 
High Current Isolator and an A310 Accupulser Signal Generator (both from World Precision Instruments, Sarasota, FL, USA; www.wpiinc.com). Stimulation was either performed for 2 seconds or 30 seconds, to release the readily releasable pool (RRP) or the recycling pool vesicles, respectively. Images were acquired at 3.3 frames per second with $100 \mathrm{~ms}$ exposure time using the Olympus setup (see 2.3.1.1), equipped with the $40 \mathrm{x}, 0.75 \mathrm{NA}$ objective. To enable fast data transfer to the computer, images were acquired with $2 \times 2$ pixel binning. The pHluorin was imaged with the Alexa 488 filter, the Synaptotagmin (Oyster550) was imaged with the TRITC filter (see Table 2-3 for filter specifications).

PHluorin data analysis was performed largely as described (Mitchell and Ryan, 2004). Briefly, those boutonal areas increasing in fluorescence during stimulation were identified as active boutons for analysis. The change in fluorescence of these areas was calculated for each frame as the difference between the fluorescence in the particular frame and the initial (pre-stimulation) fluorescence (delta $F$ or $d F$ ). This $d F$ was normalized by dividing by the initial fluorescence, yielding $\mathrm{dF} / \mathrm{F}_{0}$.

The maximum pHluorin response to ammonium chloride treatment (by puffing of a $100 \mathrm{mM}$ ammonium chloride in phosphate buffered saline [PBS: $150 \mathrm{mM} \mathrm{NaCl}, 20$ $\mathrm{mM} \mathrm{Na} 2 \mathrm{HPO}_{4}, \mathrm{pH} 7.4$ ]; resulting $\mathrm{NH}_{4} \mathrm{Cl}$ concentration of $\sim 70 \mathrm{mM}$ ) was obtained as a measure of the total pool of pHluorin molecules. As the $\mathrm{NH}_{4} \mathrm{Cl}$ eliminates any $\mathrm{pH}$ differences between intracellular organelles and extracellular medium, all pHluorin molecules are de-quenched and become brightly fluorescent. By dividing the $d F / F_{0}$ traces by the total pHluorin pool measurements (from $\mathrm{NH}_{4} \mathrm{Cl}$ application), we obtained a trace of the fraction of pHluorin molecules mobilized with respect to the initial surface expression. As pHluorin bleaching caused a gradual shift in baseline fluorescence even in absence of pHluorin quenching/de-quenching, we first fitted the final poststimulation frames by linear regression (the initial pre-stimulation frames for Syntaxin 1; stronger bleaching due to higher surface expression causes different bleaching behavior after initial bleaching here). The obtained bleaching coefficient was then used to correct the pHluorin traces for bleaching.

To determine the fraction of the individual pHluorins present on the surface (in the plasma membrane), we needed to solve the following equation:

surface fraction $=\frac{\mathrm{F}_{\mathrm{pH7.4}}-\mathrm{R}_{\mathrm{q} / \mathrm{d}}{ }^{*} \mathrm{~F}_{\mathrm{NH}_{4} \mathrm{Cl}}}{\left(1-\mathrm{R}_{\mathrm{q} / \mathrm{d}}\right){ }^{*} \mathrm{~F}_{\mathrm{NH}_{4} \mathrm{Cl}}}$,

where $\mathrm{F}_{\mathrm{pH} 7.4}$ is the steady state fluorescence at neutral $\mathrm{pH}$ (with contributions from both de-quenched pHluorin on the surface and quenched pHluorin inside acidic organelles), 
$R_{q / d}$ is the intensity ratio of quenched over de-quenched pHluorin (a property of the pHluorin) and $\mathrm{F}_{\mathrm{NH} 4 \mathrm{Cl}}$ is the fluorescence upon $\mathrm{NH}_{4} \mathrm{Cl}$ application (which de-quenches all pHluorin). Note that this formula reports a surface fraction of 1 if $\mathrm{F}_{\mathrm{pH} 7.4}=\mathrm{F}_{\mathrm{NH} 4 \mathrm{Cl}}$ (which is true if all pHluorin is on the surface and thus de-quenched), and a surface fraction of 0 if $\mathrm{F}_{\mathrm{pH7.4}}=\mathrm{R}_{\mathrm{q} / \mathrm{d}} * \mathrm{~F}_{\mathrm{NH} 4 \mathrm{Cl}}$ (which is true if all pHluorin is in internal organelles and thus quenched). As $\mathrm{F}_{\mathrm{NH} 4 \mathrm{Cl}}$ and $\mathrm{F}_{\mathrm{pH} 7.4}$ can be directly measured in our experiments (see above), we only needed to determine $\mathrm{R}_{\mathrm{q} / \mathrm{d}}$ in order to be able to calculate the surface fraction of our pHluorins. To this end, we sequentially measured the fluorescence of flat oligodendrocyte processes expressing SynaptopHluorin at pH 5.5 and pH 7.4, and found $\mathrm{R}_{\mathrm{q} / \mathrm{d}}$ to be $0.097 \pm 0.019$ (mean \pm standard error of the mean [SEM]). This procedure is valid, as these processes do not contain any intracellular (vesicular) SynaptopHluorin contributing to the signal, so that the measured change in fluorescence can be solely attributed to the pH-dependence of the pHluorin on the surface of these processes. Using the formula above we could then determine the surface fraction of each of our pHluorins.

\subsection{Photo-oxidation Electron Microscopy}

Hippocampal cultures (9-13 DIV; grown as above but plated on Thermanox coverslips, Plano GmbH, Wetzlar, www.plano-em.de) were incubated with the amphiphilic dye FM 1-43 (10 $\mu \mathrm{M}$ in normal Tyrode: $124 \mathrm{mM} \mathrm{NaCl}, 5 \mathrm{mM} \mathrm{KCl}, 2 \mathrm{mM} \mathrm{CaCl}-$ 2, $1 \mathrm{mM} \mathrm{MgCl}$, $30 \mathrm{mM}$ glucose and $25 \mathrm{mM}$ HEPES, pH 7.4) for 10 seconds before stimulating at $20 \mathrm{~Hz}$ for 2 seconds (the RRP stimulus), using the same stimulator as above. After stimulation, the cultures were allowed to rest for various intervals 44,10 and 30 seconds), before fixation by plunging in ice-cold glutaraldehyde (2.5\% in PBS).

The diaminobenzidine (DAB) incubation, photo-oxidation and electron microscopy processing were performed according to Rizzoli and Betz (Rizzoli and Betz, 2004) as follows. The samples were washed $2 \times 10$ minutes in PBS, quenched with 100 $\mathrm{mM} \mathrm{NH} \mathrm{NH}_{4} \mathrm{Cl}$ in PBS for 20 minutes and washed for 10 more minutes in PBS before incubation with diaminobenzidine $(1.5 \mathrm{mg} / \mathrm{ml})$ for $30-45$ minutes (all steps at $4^{\circ} \mathrm{C}$ ). The DAB was replaced for fresh DAB and illumination was started using an Alexa 488 filter set (Table 2-3) and the Olympus 20x, 0.5 NA objective (Table 2-4). The formation of the electron-dense precipitate can be monitored both in brightfield and in fluorescence (see Figure 2-1). We typically allowed a dark brown precipitate to form over a period of about 45 minutes. 


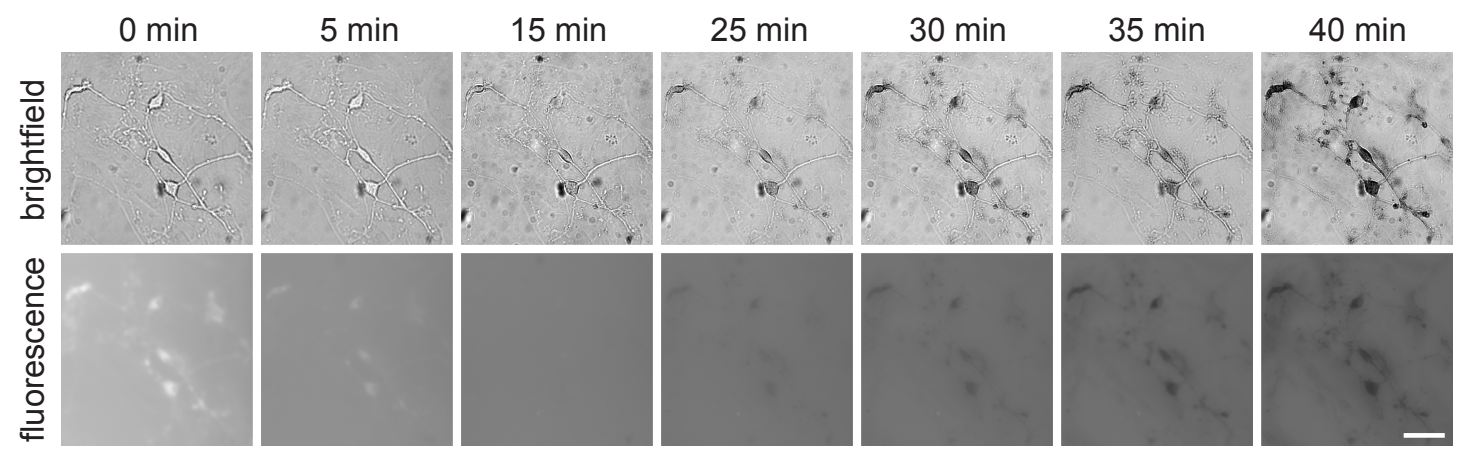

Figure 2-1: Diaminobenzidine photo-oxidation of neurons labeled with FM 1-43

Hippocampal neurons were labeled with FM 1-43 and fixed with glutaraldehyde. Neurons were then incubated with diaminobenzidine and illuminated through the Alexa 488 filter set (Table 2-3) and a 20x, 0.5 NA objective (Table 2-4). Brightfield (top panels) and fluorescence (bottom panels) images were acquired to visualize the formation of the electron dense diaminobenzidine precipitate (black). The precipitate first becomes apparent around 20 minutes after the beginning of illumination. Note that the fluorescence is completely bleached before any dark precipitate appears. Scale $=50 \mu \mathrm{m}$.

Following photo-oxidation, the samples were placed in PBS to excise the photooxidized region from the plastic coverslip. The PBS was then replaced with $400 \mu \mathrm{l}$ of $1 \%$ osmiumtetroxide $\left(\mathrm{OsO}_{4}\right)$ in $\mathrm{PBS}$ and incubated for one hour at room temperature. The $\mathrm{OsO}_{4}$ was removed and the samples were washed $2 \mathrm{x} 5$ minutes with PBS. The samples were dried by serial ethanol dehydration (30\% EtOH for 5 minutes; $50 \%$ EtOH for 5 minutes; 70\% EtOH for 5 minutes; 90\% EtOH for 10 minutes; 95\% EtOH for 2x 10 minutes; $100 \%$ EtOH for $3 \times 10$ minutes) and infiltrated with a 1:1 dilution of Epon and 100\% EtOH over night. The 50\% Epon was replaced with 100\% Epon for at least 6 hours before placing the samples to dry / cure over night ( 18 hours) at $60^{\circ} \mathrm{C}$. Samples were finally cut into thin sections and imaged with the transmission electron microscope (2.3.3).
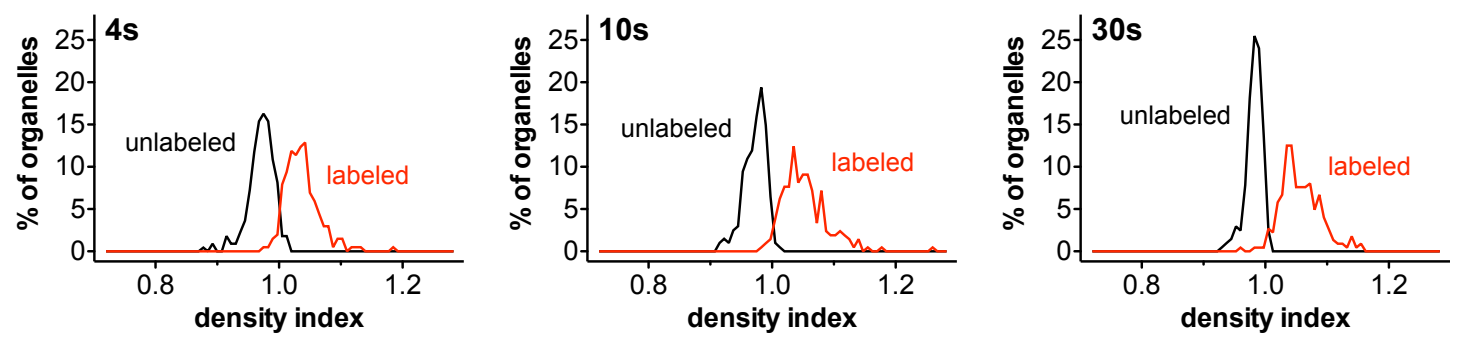

Figure 2-2: Relative density of labeled and unlabeled vesicles after photo-oxidation.

The relative density is the ratio between the density of the vesicle lumen and the vesicle membrane, which is less than 1 for unlabeled vesicles (black) and greater than 1 for labeled vesicles (red) (Rizzoli and Betz, 2004). Note that unlabeled and labeled vesicles can be easily distinguished for all time points of post-stimulation incubation, as the two distributions are always well differentiated. The graphs show histograms from at least 200 vesicles for each condition. 
No post-staining of the sections was performed, allowing for unambiguous identification of labeled vesicles for all three time points. We determined the relative density of vesicles identified as labeled or unlabeled by measuring the ratio of the density of the vesicle lumen over the density of the vesicle membrane (Rizzoli and Betz, 2004). These measurements confirmed our identification of labeled vesicles, as the density ratio distributions of labeled and unlabeled vesicles showed minimal overlap (see Figure 2-2). The vesicle diameters were determined by manually drawing line scans on the images, using a routine written in Matlab (The MathWorks Inc., Natick, MA, USA; www.mathworks.com).

\subsection{Quantum Dot Imaging and Analysis}

Hippocampal cultures (grown as above) were incubated with $5 \mu \mathrm{g} / \mathrm{ml}$ antiSynaptotagmin lumenal domain antibodies (coupled to biotin; see Table 2-1), for 7 minutes on ice in normal Tyrode buffer. After a brief wash, the antibodies were labeled for 7 minutes with streptavidin-coated quantum dots (Qdots; Invitrogen), using a mixture of Qdot 525, Qdot 565, Qdot 605 and Qdot 655, each at a concentration of 10 $\mathrm{nM}$. The Qdots were washed-off, the preparations were brought to room temperature and RRP stimulation was performed (20 Hz for 2 seconds, as above). As in the photooxidation experiments, the samples were fixed by plunging in ice-cold $4 \%$ paraformaldehyde after on interval of 4,10 or 30 seconds after stimulation. The preparations were quenched with $100 \mathrm{mM} \mathrm{NH}_{4} \mathrm{Cl}$ and finally mounted in Mowiol $(6 \mathrm{~g}$ glycerol AR, 2.4 g Mowiol ${ }^{\circledR}$ 4-88 (Merck), 6 ml H2O, 12 ml 0.2 M Tris, pH 7.2; Willig et al., 2006), before the imaging was performed with the Leica STED setup (see 2.3.2.1) in confocal mode. The excitation for all Qdots was performed at $488 \mathrm{~nm}$ and the emission light was detected at the appropriate $20 \mathrm{~nm}$ wavelength windows (515-535 nm, 555$575 \mathrm{~nm}, 595-615 \mathrm{~nm}$ and 645-665 nm, set via AOTF) using PMTs.

The images were analyzed by custom-written Matlab routines as follows. First, the position (center of mass) of each Qdot was determined with sub-pixel accuracy, followed by measurement of the distance to its closest neighbor in any of the four color channels (exactly as described, Geumann et al., 2008). The use of four spectrally different Qdots is advantageous for these experiments because of the limited spatial resolution provided by fluorescence microscopy (Abbe, 1873): two objects closer to each other than the diffraction limit of light cannot be resolved unless measured separately. This could be achieved for example by measuring the objects at different time points (Betzig et al., 2006; Rust et al., 2006) or in different colors (see discussion in 
Geumann et al., 2008). Here, the use of four spectrally separable Qdots allows distance measurements beyond the limitations of diffraction-limited optics, at least when measuring distances between Qdots localized in different color channels; thus, the more color channels are used, the more distances can be measured. Although in principle we could combine even more than four Qdots (due to their narrow emission spectra), the possible number of different Qdots is limited by the number of PMTs provided by the microscope (four in our case).

\subsection{Rab5-GFP Expression and Imaging}

\subsubsection{Endosomal trafficking in PC12 cells}

PC12 cell culture, endocytic probes and data analysis were as described before (Bethani et al., 2009). Briefly, PC12 cells were transfected with wild-type or dominant active (Q79L) Rab5-GFP using Lipofectamin 2000 (Invitrogen) according to the supplier's protocol. One day after transfection, the cells were briefly washed with PC12 saline (130mM NaCl, 4mM KCl, 48mM glucose, 10mM HEPES, 5mM CaCl2, 1mM MgCl2, pH 7.3) to remove the culture medium. Afterwards, the cells were labeled with various endocytic probes: For Transferrin (Tf) uptake, the cells were incubated for 5 minutes at $37^{\circ} \mathrm{C}$ with $50 \mu \mathrm{g} / \mathrm{ml}$ Tf-Alexa594. For Tf release, the cells were labeled in the same fashion, but were then briefly washed twice with PC12 saline and afterwards incubated at $37^{\circ} \mathrm{C}$ for 10 more minutes in the absence of Tf. For low-density lipoprotein (LDL) uptake, the cells were incubated for 5 minutes at $37^{\circ} \mathrm{C}$ with $5 \mu \mathrm{g} / \mathrm{ml}$ LDL-DiI. For dextran uptake, the cells were incubated for 5 minutes at $37^{\circ} \mathrm{C}$ with $1 \mathrm{mg} / \mathrm{ml}$ dextranAlexa594. For cholera toxin beta subunit (CTxB) uptake, the cells were pre-incubated for 30 minutes on ice with $10 \mu \mathrm{g} / \mathrm{ml}$ CTxB-Alexa647; after briefly washing 3 times with PC12 saline, the cells were incubated at $37^{\circ} \mathrm{C}$ for 40 minutes in absence of CTxB, to allow the toxin to reach the Golgi. Finally, all preparations were briefly washed twice, fixed in 4\% paraformaldehyde (in PBS), quenched with $100 \mathrm{mM} \mathrm{NH}_{4} \mathrm{Cl}$ (in PBS) and embedded in Dako mounting medium (Dako Denmark A/S, Glostrup, Denmark; www.dako.com) for imaging.

Data analysis was performed using custom-written Matlab routines, as follows. A threshold was applied to identify the Rab5-GFP transfected (green) cells. The intensity of endocytic label (red) in transfected cells was determined and normalized to the intensity in untransfected cells on the same coverslips. 


\subsubsection{Rab5-GFP expression and confocal Imaging}

Hippocampal cultures were prepared as above and were transfected between 7 and 13 DIV with the Rab5-GFP constructs (wild-type or the dominant active Q79L mutant) using NeuroMag para-magnetic beads (OZ biosciences) according to the supplier's protocol. Alternatively, we used lipofectamin transfection (Lipofectamin 2000, Invitrogen) or $\mathrm{CaPO}_{4}$ transfection (according to Xia et al., 1996). The different transfection protocols were used to test for method-specific errors. As no substantial differences could be observed in either the density of GFP-positive organelles, or the general neuronal morphology, all results were pooled. 2-3 days after transfection, the preparations were incubated at $37^{\circ} \mathrm{C}$ for 5 minutes in presence of antibodies against the lumenal (exposed) domain of Synaptotagmin I (604.2, see Table 2-1). Afterwards, the preparations were fixed ( $4 \%$ paraformaldehyde for 45 minutes), permeabilized $(0.1 \%$ triton X-100 for 15 minutes) and incubated with secondary antibody (goat anti-mouse coupled to Atto $647 \mathrm{~N} ; 1$ hour in a humidified chamber) to visualize the antiSynaptotagmin antibody. Alternatively, a directly labeled anti-Synaptotagmin antibody (604.2 coupled to Atto 647N; see Table 2-1) was used.

In a different set of experiments, neurons grown and transfected in the same fashion were incubated in presence of the same antibody on ice (to block endocytosis, see Figure 3-15) for 5 minutes (compare Willig et al., 2006). The antibody was briefly washed off and the samples were brought to room temperature, before applying an RRP releasing stimulus (20 Hz / 2 seconds) or a recycling pool releasing stimulus $(20 \mathrm{~Hz} / 30$ seconds) as above. After a resting period of 10 seconds (to allow transit of labeled vesicles to endosomes; compare with section 2.7), the samples were fixed and further processed as above, followed by thin sectioning as below (section 2.9.3).

Conventional confocal imaging was performed with the Leica STED setup (see section 2.3.2.1) in confocal mode. Z-stacks were acquired through the endosomes at 300 nm Z-intervals. Excitation was achieved with the $488 \mathrm{~nm}$ Argon laser line (for GFP) and the $633 \mathrm{~nm}$ HeNe laser (for Atto 647N), emission was detected with PMTs.

\subsubsection{Thin section STED imaging}

Sample processing for plastic embedding and thin sectioning was performed exactly as previously described (Punge et al., 2008). Imaging was performed with the Leica STED setup (see section 2.3.2.1). For STED imaging, excitation was performed with the 635 $\mathrm{nm}$ diode laser, depletion was achieved with the MaiTai tunable laser at $750 \mathrm{~nm}$ and the signal was detected with an APD. The system resolution limit is $\sim 70-80 \mathrm{~nm}$, measured by analysis of crimson-fluorescent beads ( $20 \mathrm{~nm}$ diameter, purchased from Invitrogen). 
GFP signal was imaged by immunostaining the GFP moiety using the rabbit polyclonal ab290 antibody (Abcam; see Table 2-1), detected via Cy3-labeled secondary antibodies. This step is necessary, as the GFP fluorescence is not preserved after plastic embedding. Imaging of the Cy3 fluorescence was performed in confocal mode, using the $543 \mathrm{~nm}$ HeNe laser line (Table 2-5) for excitation and a PMT for detection.

Data was analyzed using custom-written routines in Matlab, as follows. Rectangular regions of interest (ROIs) were selected in areas containing endosomes (identified in the GFP channel). The area covered by endosomes (green) was determined by applying an automatic threshold 1.5-fold higher than the mean intensity of the ROI (which was empirically determined to function well for our ROIs). A similar threshold was applied to the Synaptotagmin channel (red) and the amount of overlap was determined as the relative area of endosomes covered by vesicle staining (relative number of pixels). The values were normalized to a positive colocalization control, which was obtained by double immunostaining with two anti-Synaptotagmin antibodies (604.2 coupled to Atto $647 \mathrm{~N}$ and 604.2 coupled to Oyster550; see Table 2-1), before fixation and thin-section processing, as above (see also: Punge et al., 2008; Opazo et al., 2010). The imaging for this control was performed exactly as above; the overlap between the STED (Atto 647N) Synaptotagmin signal and the confocal (Oyster550) Synaptotagmin signal was $27.55 \pm 2 \%$ (mean \pm SEM of three independent experiments) of the surface occupied by the confocal signal. This relatively low overlap is to be expected, as the lower resolution in the Oyster550 imaging (single spot Full width at half maximum $[\mathrm{FWHM}] \sim 267 \mathrm{~nm}$ ) renders the spots substantially larger than the Atto $647 \mathrm{~N}$ spots. Due to a change in image quality for the experiments with electrical stimulation (Figure 3-9 D), the thresholding had to be slightly adjusted, resulting in an overlap of $22.62 \pm 3.3 \%$ (mean \pm SEM of three independent experiments) for the colocalization control experiment.

\subsubsection{Live STED imaging and analysis}

Hippocampal cultures were grown and transfected with the dominant active (Q79L) Rab5-GFP mutant as above. Synaptic vesicles were labeled with directly labeled antiSynaptotagmin antibodies (604.2 directly labeled with Atto 647N; see Table 2-1) in normal Tyrode buffer for 5 minutes on ice. Antibodies were quickly washed-off before imaging at 28 frames per second. The imaging was performed using a live STED setup, as described (Westphal et al., 2008). A second excitation/emission channel was implemented allowing for simultaneous Rab5-GFP imaging (see 2.3.2.2 for details). The 
samples were either imaged at rest or during a 30 second stimulation at $20 \mathrm{~Hz}$ (the recycling pool stimulus; stimulation was performed as in section 2.6.3).

Data was analyzed using custom-written routines in Matlab, as follows. Summed images were obtained by adding all frames of the movies. Naturally, this procedure blurs the images, but provides an overall impression of the areas occupied by endosomes and vesicles during the entire acquisition time. Rectangular ROIs were selected in the areas occupied by the endosomes and the Pearson's correlation coefficients were calculated between the ROIs in the green (endosome, GFP) and red (vesicles, Synaptotagmin) channels.

\subsection{Blocking Endosome Function with Soluble Syntaxin 13 Fragment}

\subsubsection{Syntaxin 13 expression, imaging and analysis}

Hippocampal cultures were grown as above. Soluble Syntaxin 13 fragments (amino acids 1-250; or amino acids 1-186, a fragment also lacking the SNARE domain) were expressed from a GFP plasmid using an internal ribosomal entry site (IRES) to avoid any effects on the Syntaxin 13 fragment that would be caused by the presence of a GFP-tag (see section 1.1). As a control for general effects of transfection, we expressed GFP alone.

1-2 days after transfection, the cells were used for various staining protocols to determine the sizes of vesicle pools. To determine the total amount of vesicles, the cultures were directly fixed and immunostained against Synaptophysin (using the G96 serum (see Table 2-1) and Cy3-conjugated antibodies for secondary detection). Alternatively, the preparations were stimulated at $20 \mathrm{~Hz}$ in presence of $10 \mu \mathrm{M} \mathrm{FM} \mathrm{4-}$ 64FX (Invitrogen), either for 30 seconds to label the recycling pool vesicles, or for 2 seconds to label only the RRP. Both of these preparations were allowed to recycle vesicles for another 30 seconds before fixation. To test whether the labeled RRP vesicles can be re-released, the preparations were washed for 10 minutes and subsequently subjected to a second stimulation train (20 Hz / 30 seconds), before continuing with fixation. Finally, to label spontaneously releasing vesicles, the cells were incubated with $10 \mu \mathrm{M}$ FM 4-64FX in presence of $1 \mu \mathrm{M}$ tetrodotoxin (TTX; to block action potential evoked synaptic vesicle release) for 15 minutes, directly followed by fixation. After fixation (and secondary Cy3 immunostaining for the total pool samples), all preparations were embedded in Mowiol and imaged with the Leica STED setup (see 2.3.2.1) in confocal mode. The Argon $488 \mathrm{~nm}$ and the HeNe $543 \mathrm{~nm}$ laser lines (Table 2-5) were used for GFP and FM 4-64FX / Cy3 excitation, respectively. 
The intensity of the vesicle labeling (immunostaining or FM-dye staining) was determined by custom-written routines in Matlab, as follows. Rectangular ROIs were selected in the GFP channel and the GFP-positive boutons (expressing either GFP alone or GFP together with the soluble Syntaxin 13 fragment) were determined by applying an automatic threshold. The average intensity of vesicle labeling in the corresponding areas was calculated and the data was normalized to the GFP only control.

\subsubsection{Monte Carlo model of RRP recycling}

A Monte Carlo model of RRP recycling was generated to determine the effects of endosomal inhibition on the fusion competence of RRP vesicles. We assumed that endocytosis of fused vesicles can lead to the retrieval of "dirty" vesicles - vesicles containing also plasma membrane material; these vesicles would normally be sorted by the endosome, so that inhibition of endosomal function would lead to an accumulation of "dirty" vesicles, at the expense of the RRP. To estimate the reduction in release probability of unsorted "dirty" vesicles in absence of endosomal sorting, we simulated the resulting RRP size of several scenarios of assumed release probabilities.

The parameters to construct our model were either taken from the literature or from this study as follows. The RRP inside the synapse was set to comprise 20 vesicles $\left(\mathrm{n}_{\mathrm{n}}\right)$ (Schikorski and Stevens, 2001) and an equal-sized pool of fused (readily retrievable) vesicles $\left(\mathrm{nf}_{\mathrm{n}}\right)$ was set to be in equilibrium with the RRP (Wienisch and Klingauf, 2006). Initially, neither "dirty" $\left(n_{d}\right)$ nor fused "dirty" vesicles $\left(n_{d}\right)$ were assumed to be present in the synapse. The probability of release for the normal vesicles $\left(\mathrm{pr}_{\mathrm{n}}\right.$ ) was set to 0.3 (Gandhi and Stevens, 2003), increasing with tetanic stimulation ( $\geq 5$ $\mathrm{Hz}$ ) to reach a probability of 1 at a stimulation frequency of 20-30 Hz. For the different models, our parameter of interest, the probability of release for the "dirty" vesicles $\left(\mathrm{pr}_{\mathrm{d}}\right.$ ), was set to a variable fraction of $\mathrm{pr}_{\mathrm{n}}\left(\mathrm{pr}_{\mathrm{n}} / \mathrm{pr}_{\mathrm{d}}\right.$ is plotted on the $\mathrm{Y}$ axis in Figure 3-14 B). The probability of spontaneous release of the normal vesicles $\left(\mathrm{ps}_{\mathrm{n}}\right)$ was set to once per minute (Sara et al., 2005); this value was adjusted to a higher level for the "dirty" vesicles, according to the results from Figure 3-13 D. The probability that a normal vesicle is endocytosed $\left(\mathrm{pe}_{\mathrm{n}}\right)$ was considered equal to the initial probability of release (0.3), generating an accurate compensatory retrieval mechanism. The increased release probability during tetanic stimulation was compensated for by prolonged compensatory endocytosis - i.e. the endocytosis continued at the same probability (0.3), resulting in a transient increase in surface membrane. For simplicity, spontaneously released vesicles were assumed to be retrieved with identical probability of endocytosis. The probability of endocytosis for the "dirty" vesicles ( $\mathrm{pe}_{\mathrm{d}}$ ) was set equal to that of the normal vesicles, 
as both would theoretically be targeted by the same machinery (especially as both "dirty" and normal vesicles are retrieved simultaneously, Figure 3-18). The probability that a retrieved vesicle was/became "dirty" was set to 0.4 , representing the proportion of newly retrieved vesicles enriched in plasma membrane components (Figure 3-18 D, SNAP-25 and Syntaxin 1).

The simulations were then performed as follows. A train of 20 action potentials (at variable frequencies for the different models) was delivered and followed by a resting period of 30 seconds to allow for compensatory endocytosis. Afterwards, endosomal function was inhibited and a prolonged period of activity (1000 action potentials at $1 \mathrm{~Hz}$ ) was allowed to equilibrate the vesicle pools and generate "dirty" vesicles. The amount of release obtained from a subsequent second train of 20 action potentials was expressed as a fraction of the response to the first stimulus (mapped to different colors in Figure 3-14 B), and thus represents the effect of endosomal inhibition on the RRP size.

\subsection{Synaptic Vesicle Marker Surface Immunostaining}

Hippocampal cultures were prepared as above and were used between 8 and 34 days in vitro. Fluorescently-coupled antibodies against the lumenal (extracellular) domain of Synaptotagmin I (604.2, coupled to the dye Atto $647 \mathrm{~N}$; see Table 2-1) and a rabbit polyclonal serum recognizing a lumenal epitope of Synaptophysin (G96; see Table 2-1) were used to label the surface (fused) synaptic vesicle epitopes. To label specifically the recently exocytosed vesicles, the preparations were first incubated for 10 minutes with unlabeled 604.2 antibodies (diluted 1:50 from ascites fluids; typically $\sim 1-3 \mathrm{mg} / \mathrm{ml}$ antibody concentrations; see Table 2-1) in Tyrode buffer. Vesicle recycling was inhibited by performing the incubation either at $4^{\circ} \mathrm{C}$ or in absence of $\mathrm{Mg}^{2+}$ and $\mathrm{Ca}^{2+}$ at room temperature. After surface epitope blocking, the preparations were incubated with the fluorescently-coupled anti-Synaptotagmin antibody and the anti-Synaptophysin serum (6 minutes, depolarizing with $15 \mathrm{mM} \mathrm{KCl}$; see also Willig et al., 2006). The preparations were fixed in $4 \%$ paraformaldehyde (in PBS) for 10 minutes on ice, followed by 35 minutes at room temperature. Synaptophysin antibodies were stained by secondary antibodies coupled to the fluorescent dye Atto 590, before mounting the preparations in 2,2'-thiodiethanol (TDE). Alternatively, a positive colocalization control was performed as follows: no pre-blocking of the surface Synaptotagmin epitopes was performed and the stainings were performed without anti-Synaptophysin antibodies; secondary staining was performed with an anti-mouse secondary antibody, coupled to the 
fluorescent dye Atto 590 (thus resulting in Synaptotagmin being double-stained with Atto $647 \mathrm{~N}$ and Atto 590).

The preparations were imaged using the 2-color STED setup (see 2.3.2.3). Custom-written Matlab routines were used for measuring spot diameters from Lorentzian curve fits (Willig et al., 2006), which were generated for line scans through the STED spots. The fits were used to determine the full width at half maximum (FWHM) of the spots. The distance between intensity centers of spots was analyzed exactly as described (Geumann et al., 2008).

\subsection{Isolated Synaptic Vesicle Immunoassay}

Synaptosomes were prepared and live-stained using a fluorescently labeled antibody against the lumenal (exposed) domain of Synaptotagmin I (604.2, coupled to the dye Oyster550; see Table 2-1), exactly as described (Rizzoli et al., 2006). The LS1 fraction, enriched in synaptic vesicles (Huttner et al., 1983) was removed and snap-frozen, for long-term storage (see 2.5 for details on synaptosome preparation).

The validity of this procedure to label recycling vesicles in synaptosomes was tested by labeling synaptosomes with FM 1-43 as described (Rizzoli et al., 2006), followed by photo-oxidation and electron microscopy processing (see 2.7 for details on photo-oxidation; fixation was performed using $4 \%$ paraformaldehyde and $0.1 \%$ glutaraldehyde). Alternatively, the FM-labeled LS1 fraction was adsorbed onto glass coverslips for imaging single vesicles with the high-sensitivity Zeiss Examiner setup (see 2.3.1.3).

For single vesicle immunostaining experiments, the LS1 fraction (labeled with 604.2 Oyster550) was adsorbed onto coverslips as described (Rizzoli et al., 2006). After fixation, the vesicles were incubated sequentially with antibodies against the protein of interest, followed by fluorescently labeled secondary antibodies. Then, incubation with antibodies against a synaptic vesicle marker (typically the anti-Synaptophysin rabbit polyclonal G96, or the anti-Synaptobrevin mouse monoclonal 69.1; see Table 2-1) was followed by incubation with fluorescently labeled secondary antibodies. The preparations were mounted in fluorescent mounting medium (Dako) and imaged in epifluorescence (confocal imaging is not required as the vesicles are adsorbed as a thin monolayer or organelles; see 2.3.1.1 and 2.3.1.2 for the microscope setups used). The green (Cy2) channel was used for the protein of interest, the orange (Oyster550) channel for the lumenal anti-Synaptotagmin antibody, and the deep red (Cy5) channel for the vesicle marker. The images were aligned by use of multi-color TetraSpeck ${ }^{\mathrm{TM}}$ 
beads (as described, Geumann et al., 2008), which were unambiguously identified by their blue fluorescence. Further primary antibodies (all listed in Table 2-1) used were: anti-Synaptotagmin I (41.1), anti-Vglut1 (shigeo2), anti-GABA-transporter (R22), antiSNAP-25 (71.1), anti-Syntaxin 1 (HPC1), anti-Clathrin heavy chain (clone 23), antiDynamin 1,2,3, anti-AP180, anti-Endophilin, anti-Synaptojanin 1 (C-terminus), antiAmphiphysin, anti-Uncoating ATPase (3C5), anti- $\alpha$-SNAP (77.1), anti-NSF (83.11), antiMunc18-1 (131.1), anti- $\beta$-actin (AC-15), anti-Vti1a (103.3), anti-Syntaxin 6 (clone 30), anti-Syntaxin 13, anti-ADP Ribosylation Factor (Arf; 1D9), anti-AP1 complex subunit $\gamma-1$, anti-AP-3 $\beta$-2, anti-Rab4 (clone 7), anti-Rab5 (621.1), anti-PI3P (Z-P003), anti-HA-tag, anti-NMDA receptor (54.1) and anti-Voltage-gated $\mathrm{Ca}^{2+}$ channel.

Automated data analysis was performed using custom-written routines in Matlab (as described in detail in Hoopmann, 2008). Briefly, images were aligned and filtered by an unsharp procedure to increase signal-to-noise. The images were then subjected to a threshold to identify the spots (vesicles). Single pixels surpassing the threshold, as well as spots too large to represent single vesicles (but that were rather clumps of vesicles), were automatically eliminated from the analysis (size assessed by FWHM on the basis of Gaussian fits for line scans through the spots). The threshold was automatically adjusted, for each independent staining, by using background control coverslips, which had been treated similarly (i.e., immunostained), but without addition of vesicles. Line scans through the spots (automatically selected in the synaptic vesicle marker channel for the general pool vesicles, or in the lumenal Synaptotagmin channel for the recently endocytosed vesicles) were performed and the Pearson's correlation coefficients between the signals in the different channels were calculated. A stringent correlation coefficient cut-off of 0.9 was used for identifying colocalization the correlation coefficient ranges from -1 for perfect anti-correlation to 1 for perfect correlation of signals). The analysis readout thus reported the fraction of vesicles correlating with any of the investigated proteins of interest, for both general pool vesicles and recently endocytosed vesicles. These fractions were corrected for random antibody colocalization (obtained from the background control coverslips) and were also plotted as the change in correlating vesicles for the recently endocytosed vesicles versus the general pool vesicles (positive for an increase, negative for a decrease in the amount of correlation in the recently endocytosed vesicles. 


\subsection{Extraction of Cholesterol from Endosomes}

PC12 endosomes were prepared by sucrose gradient centrifugation from PC12 postnuclear supernatants, exactly as described (Rizzoli et al., 2006) and synaptosomal organelles were prepared as described above (see section 2.5). Organelles were centrifuged onto poly-l-lysine coated coverslips, treated with methyl- $\beta$-cyclodextrin (60 $\mathrm{mg} / \mathrm{ml}$ for 30 minutes), fixed and immunostained exactly as described (Geumann et al., 2009). Secondary antibodies coupled to Atto 590 and Atto $647 \mathrm{~N}$ were used to allow for high-resolution 2-color STED imaging, which was performed using the 2-color STED setup (section 2.3.2.3), after embedding in Mowiol. Images were analyzed by determining the Pearson's correlation coefficient for the green and red (Atto 590 and Atto $647 \mathrm{~N}$ ) staining of individual endosomes, using custom-written Matlab routines. 


\section{RESULTS}

\subsection{Endosomal SNAREs Participate in Synaptic Vesicle Recycling}

As pointed out in the introduction, this study aims at elucidating the role of endosomes in synaptic vesicle recycling. If endosomes are indeed participating in the recycling of synaptic vesicle material, it should be possible to identify endosomal molecules on at least a subpopulation of synaptic vesicles recycling via endosomes. In fact, the endosomal SNAREs Syntaxin 13 ( $\mathrm{Q}_{\mathrm{a}}$-SNARE), Vti1a ( $\mathrm{Q}_{\mathrm{b}}$-SNARE) and Syntaxin $6\left(\mathrm{Q}_{\mathrm{c}^{-}}\right.$ SNARE) have been identified earlier to be enriched on synaptic vesicles (Rizzoli et al., 2006), which is in agreement with the finding that purified recently endocytosed synaptic vesicles can fuse with purified endosomes from neuroendocrine PC12 cells in vitro (Rizzoli et al., 2006).

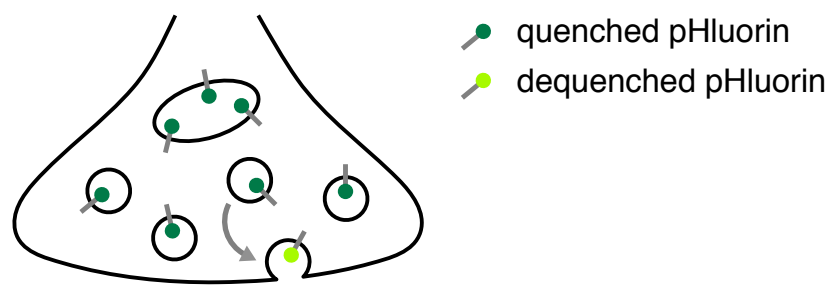

Figure 3-1: Phluorin function

The pH-sensitive GFP moiety (green) is quenched at the low pH of the acidic endosomes/synaptic vesicles (dark green). GFP fluorescence increases upon exocytosis, due to the change from acidic to neutral pH (light green), thus allowing the use of pHluorins as fusion reporters.

In order to investigate this matter in more detail, I devised an experimental approach to test directly the involvement of these endosomal SNAREs in synaptic vesicle recycling. A well-established experimental tool to study synaptic vesicle recycling are the pHluorins (Miesenbock et al., 1998), which were developed in the late 1990s. PHluorins are fusion proteins of a synaptic vesicle protein and a $\mathrm{pH}$-sensitive GFP variant (pHluorin), which is quenched at the acidic vesicular $\mathrm{pH}(\sim 5.5)$ and is brightly fluorescent at the neutral extracellular $\mathrm{pH}$ (see Figure 3-1). This property renders the pHluorins a one-of-a-kind fusion reporter that can faithfully and repeatedly indicate recycling of individual synaptic vesicles. Although pHluorins have been used in a variety of studies on vesicle recycling (see for example: Gandhi and Stevens, 2003; Mitchell and Ryan, 2004; Granseth et al., 2006; Wienisch and Klingauf, 2006), no pHluorin reporter to assay endosomal involvement in synaptic vesicle recycling has been described so far. 
We therefore generated three novel pHluorins by fusing pHluorin to the intravesicular domains of the three endosomal SNAREs Syntaxin 13, Vti1a and Syntaxin 6. For comparison purposes, we also used the well-characterized SynaptopHluorin construct (pHluorin fused to the synaptic vesicle SNARE Synaptobrevin; Miesenbock et al., 1998) and the pHluorin chimera of the plasma membrane exocytic SNARE Syntaxin 1 (Mitchell and Ryan, 2004).

\section{A}

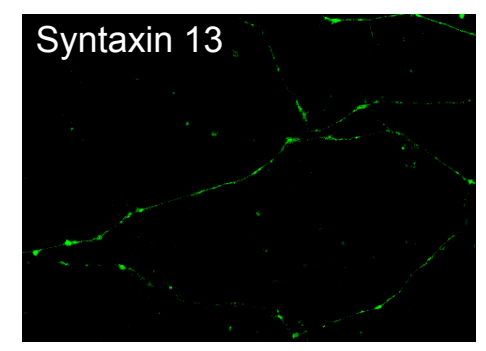

B

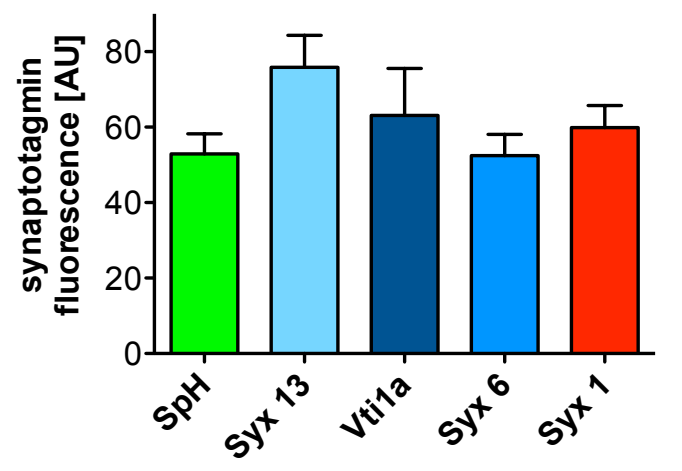

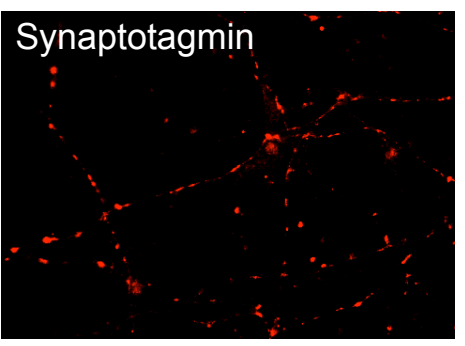

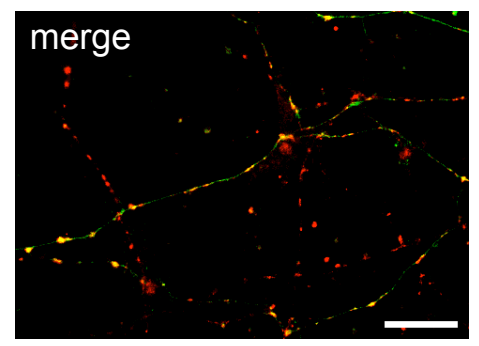

C

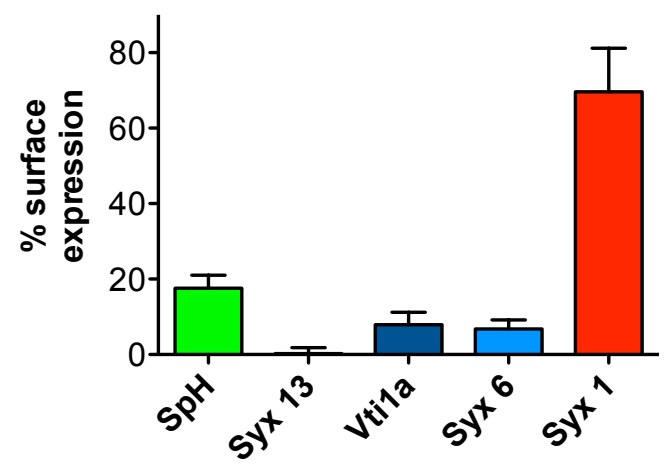

Figure 3-2: The endosomal pHluorins do not inhibit vesicle recycling

(A) Syntaxin 13-pHluorin was expressed in hippocampal cultures, which were allowed to recycle vesicles for 10 minutes in presence of an antibody against the lumenal domain of Synaptotagmin coupled to the fluorescent dye Oyster550 (see Table 2-1). Note the high correlation between Syntaxin 13-pHluorin signal (green) and Oyster550 signal (red). Scale $=25 \mu \mathrm{m}$.

(B) Quantification of the antibody uptake (as in A) for the different pHluorins. Bars show mean \pm SEM for 8 to 20 independent experiments. No change was detected between SynaptopHluorin $(\mathrm{SpH})$, the novel endosomal pHluorin constructs and Syntaxin 1-pHluorin.

(C) Surface expression for the different pHluorins, indicated as percentage of all pHluorin molecules. Bars show mean \pm SEM for 7 to 15 independent experiments (10-60 synaptic boutons were analyzed in each experiment).

When we labeled hippocampal neurons expressing the various pHluorin constructs with antibodies against the lumenal domain of Synaptotagmin (using the 604.2 antibody coupled to the dye Oyster550; see Table 2-1), we observed substantial labeling of recycling vesicles for neurons transfected with any of the tested pHluorin constructs (Figure 3-2 A-B). This finding indicates that the expression of these 
constructs did not drastically interfere with synaptic vesicle recycling in general. To assess further whether the pHluorins are properly expressed and localized, we determined the surface fraction of each pHluorin (see section 2.6.3 for calculation of surface fractions). Confirming the impression of the proper localization of our pHluorin reporters, SynaptopHluorin $(\sim 18 \%$ surface fraction, well within the previously reported range; Sankaranarayanan et al., 2000; Granseth et al., 2006) and our endosomal pHluorins $(<10 \%$ surface fraction) were predominantly present in intracellular organelles, while Syntaxin 1-pHluorin $(\sim 70 \%$ surface fraction, again similar as reported before; Mitchell and Ryan, 2004) was almost exclusively localized to the plasma membrane (Figure 3-2 C). Interestingly, the three endosomal SNARE-pHluorins show considerably low surface expression, indicative of superior localization in comparison to SynaptopHluorin, Synaptotagmin-pHluorin ( $20 \%$ surface fraction; Fernandez-Alfonso et al., 2006) and (at least for Syntaxin 13-pHluorin) even Synaptophysin-pHluorin $(\sim 8 \%$ surface fraction; Granseth et al., 2006).

Like SynaptopHluorin, all endosomal pHluorins recycled during stimulation, as shown by the fluorescence increase of individual boutons (Figure 3-3 A). When we measured the fraction of pHluorin release upon RRP stimulation $(20 \mathrm{~Hz} / 2$ seconds; releasing $\sim 10-20 \%$ of all vesicles; Schikorski and Stevens, 2001) by normalizing the $\mathrm{dF} / \mathrm{F}_{0}$ trace to the maximal response to $\mathrm{NH}_{4} \mathrm{Cl}$ application (which de-quenches all pHluorin by neutralizing vesicular $\mathrm{pH}$; see section 2.6 .3 for details), we observed a considerable response of both SynaptopHluorin and the endosomal SNAREs (Figure 3-3 B). In agreement with the fact that Syntaxin 1-pHluorin is mainly expressed on the plasma membrane (Figure 3-2 C), we could not detect a fluorescence increase upon stimulation with this construct. Conversely, some Syntaxin 1-pHluorin appeared to be endocytosed into the terminal upon stimulation, as indicated by the transient decline in pHluorin fluorescence. The fractions of endosomal pHluorins and SynaptopHluorin released upon RRP stimulation were similar and they were released with similar kinetics (as shown by scaling all traces to the peak response of SynaptopHluorin; see inset in Figure 3-3 B). While the fractional release of SynaptopHluorin and endosomal SNAREs were almost identical upon RRP stimulation, stronger recycling pool stimulation (20 Hz / 30 seconds) caused a relatively smaller response of the endosomal SNAREs (Figure 3-3 C). The release occurred again with similar kinetics, although the endosomal SNAREs seemed to release slightly slower (half-times of 12.2, 18.2 and 11.7 seconds for Syntaxin 13, Vti1a and Syntaxin 6, respectively, compared with 10.5 seconds for SynaptopHluorin; see inset of Figure 3-3 C). 
A

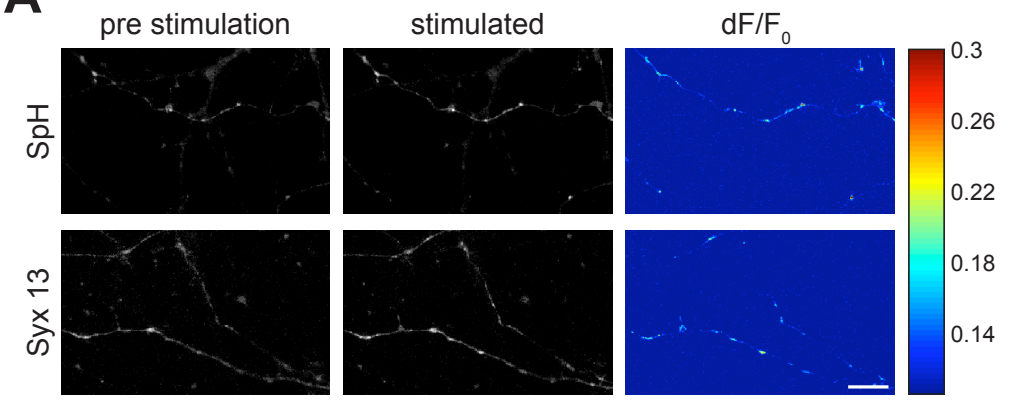

B
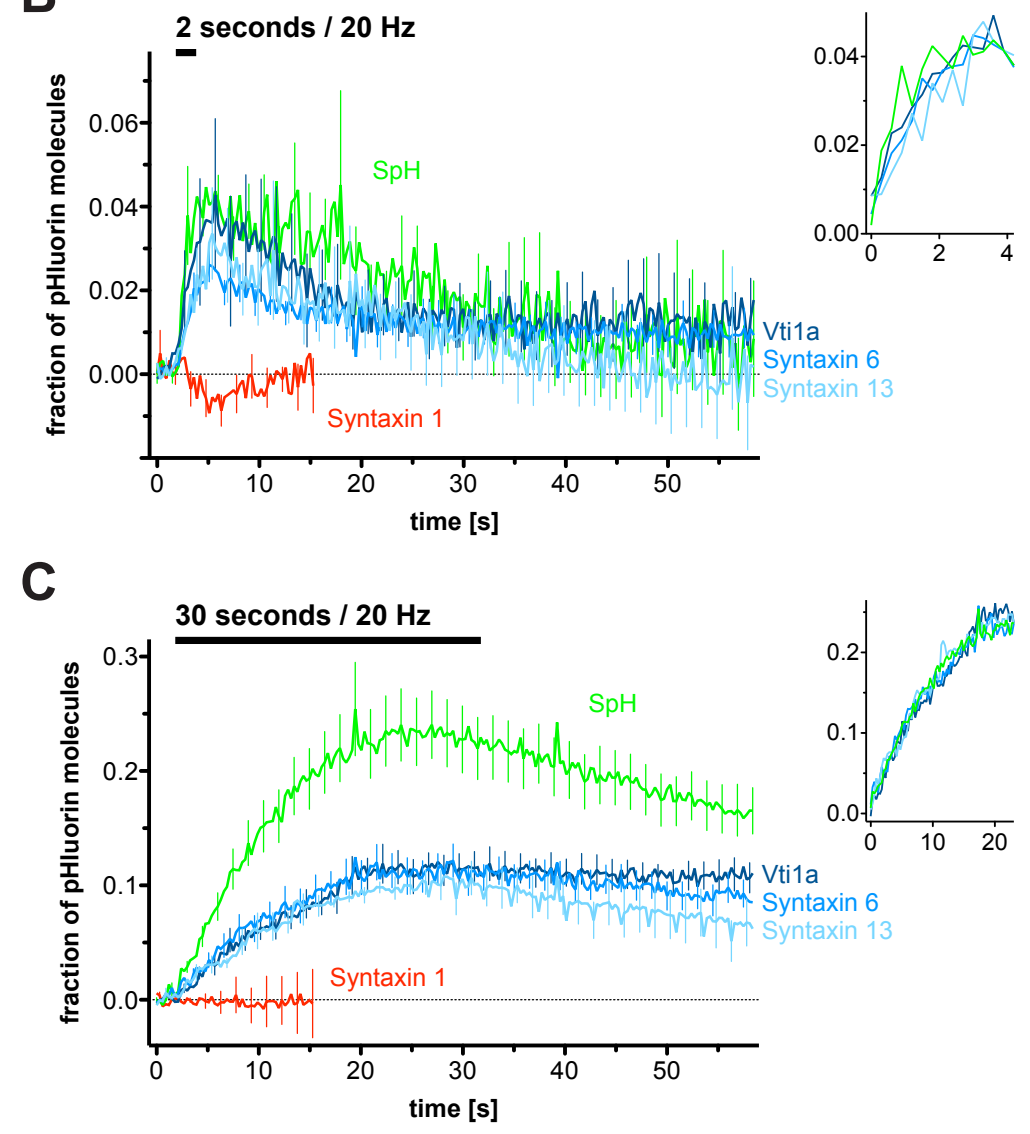

Figure 3-3: Endosomal pHluorins recycle upon electrical stimulation

(A) Synaptic boutons expressing SynaptopHluorin ( $\mathrm{SpH}$; top panels) or Syntaxin 13-pHluorin (Syx 13; bottom panels) respond to electrical stimulation at $20 \mathrm{~Hz}$. Panels on the left show resting preparations, middle panels show preparations stimulated for 17 seconds and panels on the right show the fractional change in fluorescence compared to the pre-stimulation condition $\left(\mathrm{dF} / \mathrm{F}_{0}\right)$. Scale $=15 \mu \mathrm{m}$.

(B) Change in pHluorin signal upon RRP-releasing stimuli (20 Hz / 2 seconds) expressed as the fraction of pHluorin molecules. Plots represent mean \pm SEM of 9-38 experiments. For clarity, only every $5^{\text {th }}$ error bar and only a short stretch of the Syntaxin 1-pHluorin signal are shown. The inset indicates the signal rise upon stimulation, with all traces normalized to the peak response of SynaptopHluorin; note the similar kinetics of all curves.

(C) Change in pHluorin signal upon stimuli releasing the entire recycling pool $(20 \mathrm{~Hz} / 30$ seconds). Plots represent mean \pm SEM of 5-27 experiments. Significantly more SynaptopHluorin than endosomal pHluorin is exocytosed ( $\mathrm{p}<0.0001$, Kolmogorov-Smirnov, KS, tests). The inset indicates the signal rise upon stimulation, as in B. 
The observation that the correlation between endosomal SNARE pHluorins and SynaptopHluorin decreases upon recycling pool stimulation (with respect to both fractional release and release kinetics) indicates that the endosomal SNAREs seem to be predominantly present on the fast-cycling RRP vesicles. In view of the fact that the endosomal recycling pathway has been described as a slow pathway, activated upon prolonged stimulation (Heuser and Reese, 1973), this finding was rather surprising and triggered further investigation.

\subsection{Morphological Evidence for Endosomal Fusion of RRP Vesicles}

\subsubsection{FM dye photo-oxidation and electron microscopy}

As the pHluorin experiments clearly suggested a predominant role of endosomal SNAREs in the recycling of RRP vesicles, I next attempted to gather direct morphological evidence for the fusion of recently endocytosed RRP vesicles with endosomes.

To this end, I chose to employ the technique of FM dye labeling followed by photo-oxidation and electron microscopy (Rizzoli and Betz, 2004; Opazo et al., 2010). This approach allows for labeling of actively recycling vesicles, and consequently of any organelles fusing with the labeled vesicles. Furthermore, by resorting to electron microscopy, the size of the labeled organelles can be determined very accurately, allowing the identification of fusion events even between small organelles. In order to clearly follow the fate of recently endocytosed RRP vesicles, it was necessary to establish a well-defined stimulation scheme. I used electrical field stimulation of hippocampal neurons at $20 \mathrm{~Hz}$ for 2 seconds (the RRP releasing stimulus) in presence of the styryl dye FM 1-43, which can insert into the lumenal leaflet of the fused vesicles and be taken up into retrieved vesicles (Betz and Bewick, 1992). To obtain snap-shots of the morphology of the recently endocytosed vesicles at various stages during recycling, I fixed the preparations 4, 10 or 30 seconds after the stimulus and then processed the preparations for photo-oxidation electron microscopy (Figure 3-4 A-B).

Immediately after stimulation (4s), the vesicles were small and homogeneous in size, with a diameter of $41.4 \pm 0.76 \mathrm{~nm}$ (mean \pm SEM; Figure 3-4 B-C) corresponding well to the diameter reported for small synaptic vesicles $(\sim 42 \mathrm{~nm}$; Takamori et al., 2006). At 10 seconds after stimulation, however, the amount of small vesicles was slightly diminished, giving rise to a fraction of larger vesicles, with diameters above $\sim 50$ $\mathrm{nm}$, which had disappeared again at 30 seconds after stimulation. Similar to the conclusions by Heuser and Reese (Heuser and Reese, 1973), this observation offers the 
simple interpretation that the recently endocytosed vesicles have fused to endosomes, generating larger labeled organelles at 10 seconds after stimulation; subsequently these organelles would bud new small vesicles, explaining the absence of large organelles at 30 seconds after stimulation. In agreement with this interpretation, the number of labeled organelles per time point correlates inversely with the mean size of the organelles (Figure 3-4 C-D).

In principle, these findings could also be explained by a model involving bulk endocytosis, followed by budding of small vesicles from the internalized cisternae (Takei et al., 1996). This possibility can, however, largely be excluded the fact that bulk endocytosis has been reported to be triggered by strong stimulation (for example $40 \mathrm{~Hz}$ for 10 seconds; Clayton et al., 2008) and by considering the following two observations: (1) the number of organelles at 10 seconds actually decreased (Figure 3-4 D), while appearance of large organelles by bulk endocytosis would cause an increase in the number of organelles (compare sketch in Figure 3-4 A; immediate bulk endocytosis can be excluded as well, as no larger labeled organelles are observed after 4 seconds); (2) the total amount of label (calculated from the labeled area) remains constant throughout the sampled time points (Figure 3-4 E), while bulk endocytosis after the 4 second time point would cause an increase in the amount of measured label (compare sketch in Figure 3-4 A).

Finally, I estimated the amount of RRP vesicles participating in endosomal fusion by dividing the total membrane area of the large labeled organelles present at 10 seconds by the average membrane area of a small vesicle (of $42 \mathrm{~nm}$ diameter). This yields an estimate of the amount of vesicles required to undergo homotypic fusion to account for the area of the large organelles observed after 10 seconds: $\sim 74 \%$ of all RRP vesicles. This is probably a slight overestimate, as this calculation assumes the volume of the endosome to be negligible (which is, however, not an unlikely assumption; see Murthy and Stevens, 1998). 
A
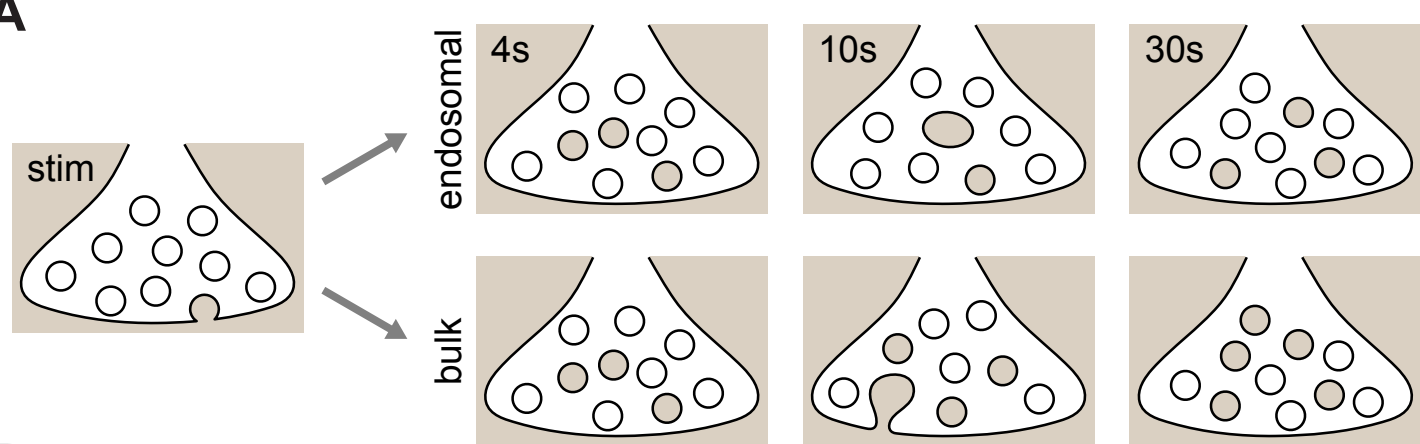

B
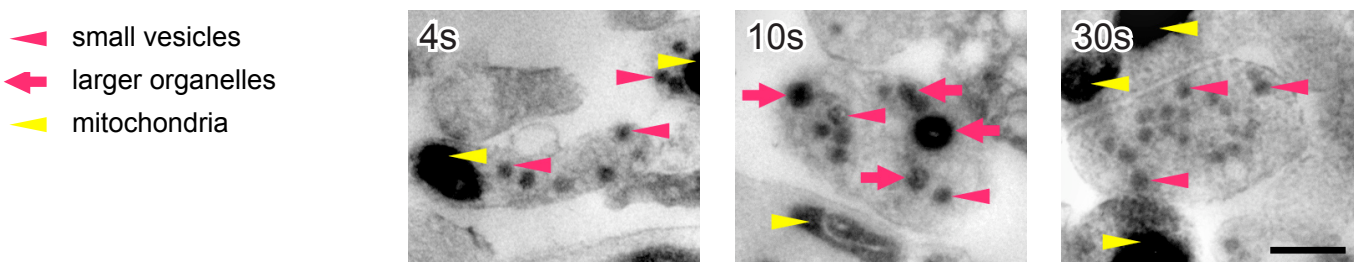

C

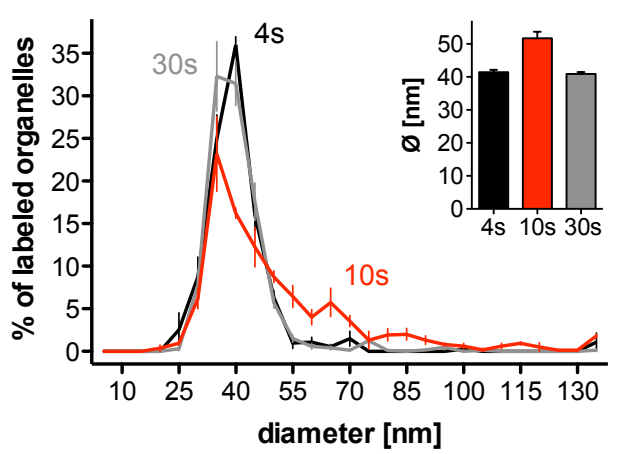

D

$\mathrm{E}$
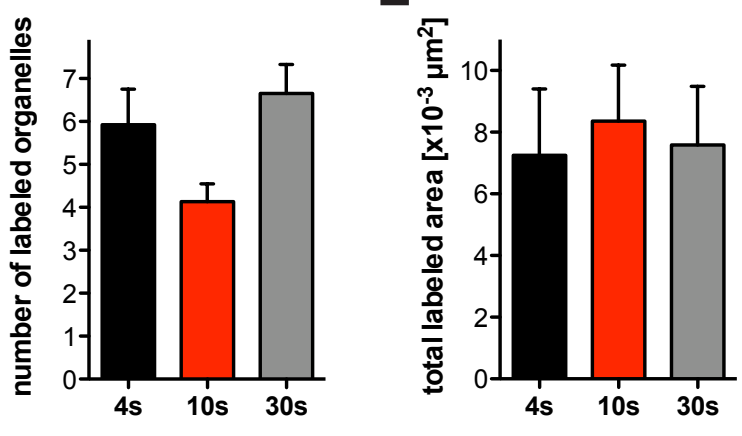

Figure 3-4: Endosomal fusion and budding of RRP vesicles

(A) Hippocampal neurons are stimulated $(20 \mathrm{~Hz} / 2$ seconds; the RRP releasing stimulus) in presence of FM 1-43, followed by fixation in ice-cold glutaraldehyde after a resting period of 4, 10 or 30 seconds. We observed a number of larger labeled organelles (see below), which could in principle be accounted for by both endosomal recycling and bulk endocytosis. In endosomal recycling (top panels) the appearance of large organelles is accompanied by a reduction in the total number of labeled objects, while the total amount of label stays constant; for bulk endocytosis (bottom panels) both the number of labeled objects and the total amount of label should increase, allowing for the differentiation between the two models.

(B) Example micrographs of nerve terminals containing organelles labeled by diaminobenzidine photo-oxidation of FM 1-43. Scale $=200 \mathrm{~nm}$. Note that mitochondria label in this procedure as well (yellow arrowheads), although this is independent of the presence of FM dyes (Grabenbauer et al., 2005). Red arrowheads/arrows point to examples of small and large vesicles, respectively; not all vesicles are indicated.

(C) Labeled vesicle size (diameter). Larger labeled vesicles ( 50-100 nm) appear after 10 seconds, and disappear after 30 seconds of rest. The size distribution at 10 seconds after stimulation is significantly different from the others $(\mathrm{p}<0.01$, KS-tests). Inset shows the average vesicle diameter (significantly larger in the 10-second condition; $\mathrm{p}<0.01$, t-tests).

(D) Number of labeled organelles per micrograph (significantly smaller in the 10-second condition; $\mathrm{p}<0.05$, t-tests).

(E) Total labeled area. The amount of endocytosed membrane remained constant in the three conditions ( $\mathrm{p}>0.1$, t-tests).

The data in (C)-(E) were obtained from three independent experiments, with 140-195 electron micrographs analyzed. All graphs show mean \pm SEM. 


\subsubsection{Qdot imaging}

Since the pHluorin experiments suggested a relation between the RRP vesicles and endosomes, and as the photo-oxidation experiments suggested a large fraction of RRP vesicles to undergo fusion to endosomes, we sought to corroborate the notion of endosomal vesicle fusion by means of light microscopy.

A

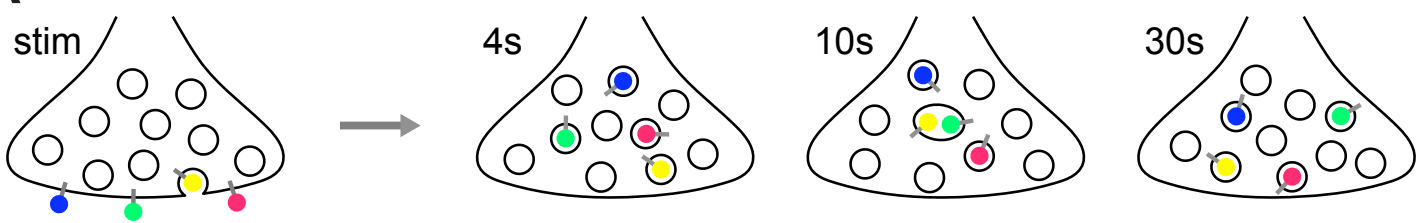

B
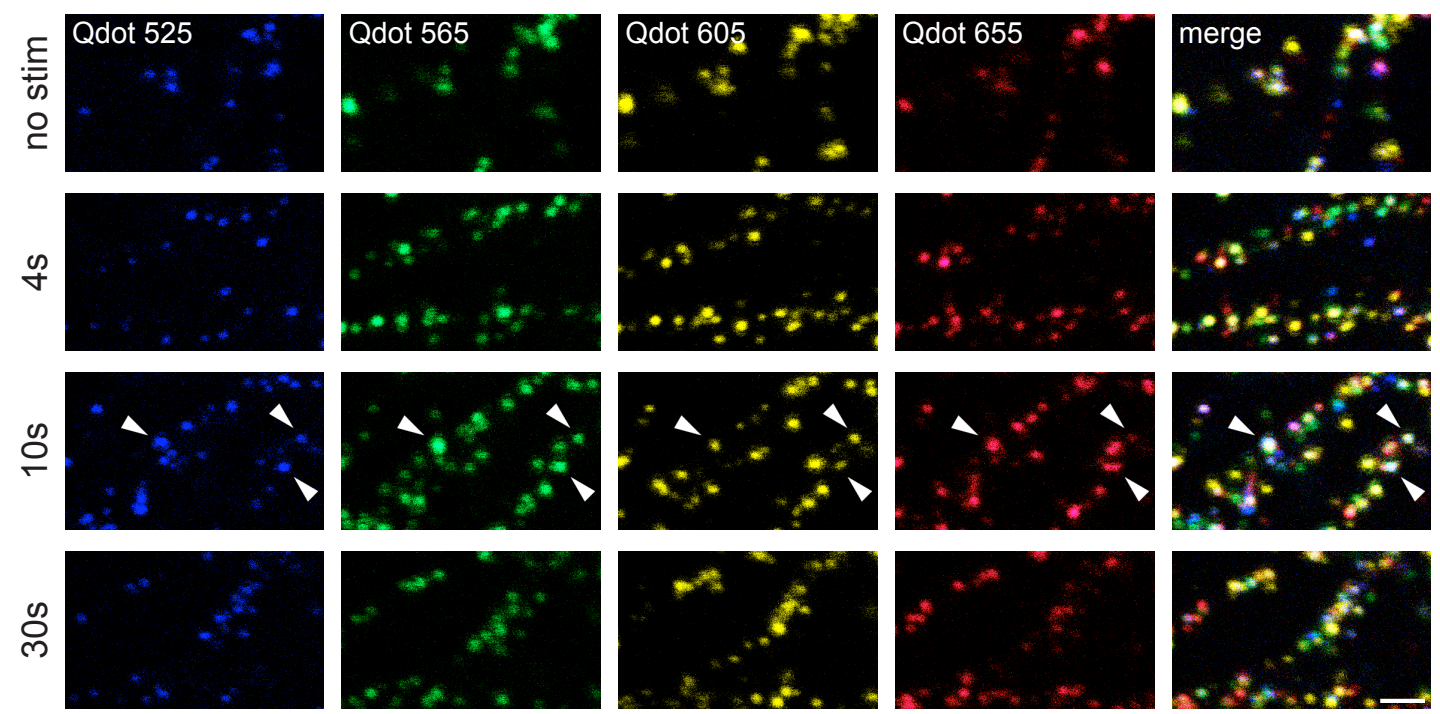

C
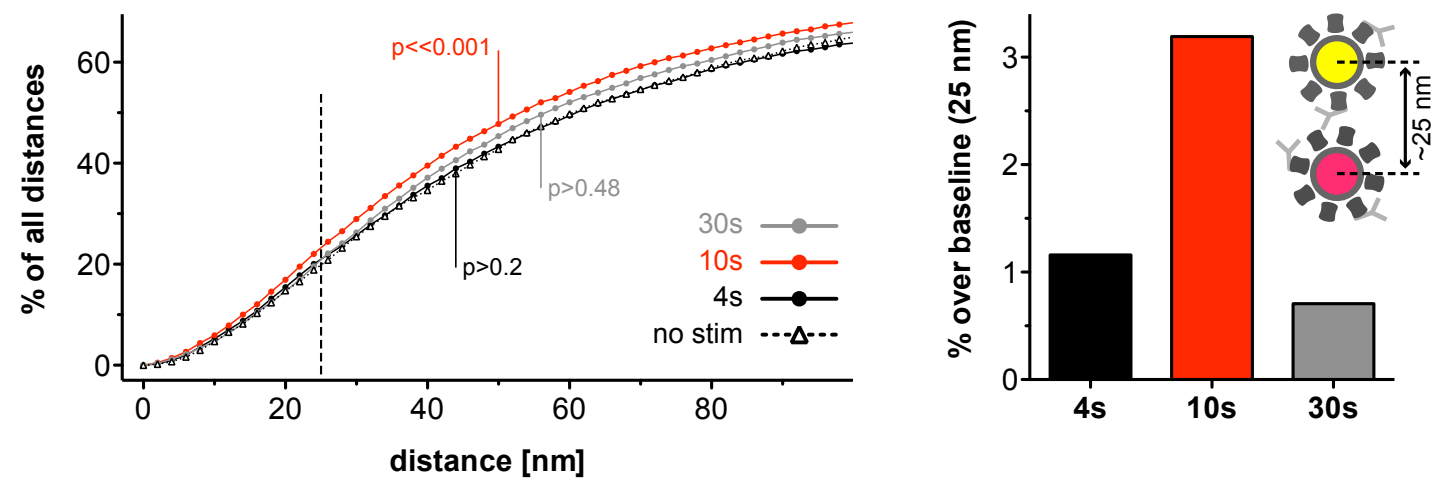

Figure 3-5: Qdots confirm endosomal fusion and budding of RRP vesicles

(A) The surface pool of Synaptotagmin is labeled with a biotinylated antibody against the lumenal domain of Synaptotagmin (see Table 2-1), followed by addition of streptavidin-coated Qdots (four different colors: Qdot 525, Qdot 565, Qdot 605 and Qdot 655). RRP stimulation (20 $\mathrm{Hz} / 2$ seconds) results in endocytosis of Qdot-labeled synaptic vesicles. If endosomal recycling is involved, multi-labeled organelles with Qdots in close proximity should subsequently appear and later disappear through budding of new vesicles. (legend continued on next page) 
(B) Typical images of Qdot-labeled nerve terminals at four different time points: before stimulation or at 4 seconds, 10 seconds or 30 seconds after stimulation. Arrowheads in the $10 \mathrm{~s}$ sample indicate example organelles of multi-color colocalization. Scale $=1.5 \mu \mathrm{m}$.

(C) The minimal distances between the differently colored Qdots were analyzed, and cumulative histograms were plotted for nerve terminals either before stimulation or at 4, 10 and 30 seconds after stimulation. The distribution at 10 seconds after stimulation is significantly different from the other three distributions ( $\mathrm{p}<<0.001, \mathrm{KS}$-tests). The distributions at 4 or 30 seconds after stimulation are neither different from each other, nor from the non-stimulated preparations ( $p>0.2$, KS-tests). The vertical dotted line indicates a distance cut-off of $25 \mathrm{~nm}$, which was used in panel (D). The histograms were obtained from $\sim 8,000-14,000$ Qdot distances, from 3 independent experiments.

(D) Amount of Qdots within less than $25 \mathrm{~nm}$ from other Qdots (cut-off as indicated by the vertical dotted line in $\mathrm{C}$ ). The inset shows the rationale of using a $25 \mathrm{~nm}$ cut-off: the diameter of the streptavidin-coated Qdots is $\sim 15-20 \mathrm{~nm}$, so that allowing for some extra space for the biotinylated antibody yields a distance of $\sim 25 \mathrm{~nm}$ between tightly apposed Qdots. The values were corrected for closely apposed Qdots before stimulation, as these are likely bound to single multi-biotinylated antibodies, and are therefore not biologically meaningful.

To reproduce the photo-oxidation experiments above using light microscopy, we turned to Qdots, which enabled us to simultaneously use four spectrally separable colors (owing to their excitability with a single $488 \mathrm{~nm}$ laser line and their narrow emission peak; see section 2.8 for details). In analogy to the photo-oxidation experiments, we used Qdots to label surface-exposed synaptic vesicles and followed their recycling. We first incubated hippocampal neurons with biotinylated antibodies against the lumenal domain of Synaptotagmin (604.2; see Table 2-1), which was followed by detection with four differently colored streptavidin-coated Qdots (Zhang et al., 2009). We stimulated the neurons exactly as above $(20 \mathrm{~Hz} / 2$ seconds; the RRP stimulus) and fixed the preparations at 4, 10 and 30 seconds after stimulation (Figure 3-5 A). The samples were then embedded and imaged with the Leica STED setup in confocal mode (see sections 2.3.2.2 and 2.8; Figure 3-5 B). We determined the distance to the nearest neighbor for each Qdot and found a significant fraction of Qdots to be tightly apposed at 10 seconds after stimulation (Figure 3-5 C-D). It should be noted that the radius of the streptavidin-coated Qdot with an attached anti-Synaptotagmin antibody measures at least about $12 \mathrm{~nm}$, resulting in a theoretical minimal distance of about $25 \mathrm{~nm}$ between two Qdots, when tightly pressed against each other. When we measured the amount of Qdots within this distance of each other (threshold of $25 \mathrm{~nm}$; vertical dotted line in Figure 3-5 C), we found a significantly larger fraction at 10 seconds than at 4 and 30 seconds (Figure 3-5 D). The observed increase in tightly apposed Qdots at 10 seconds (and the following decrease at 30 seconds) is in good agreement with endosomal fusion (and subsequent budding), verifying the results from the photo-oxidation experiments. 
A

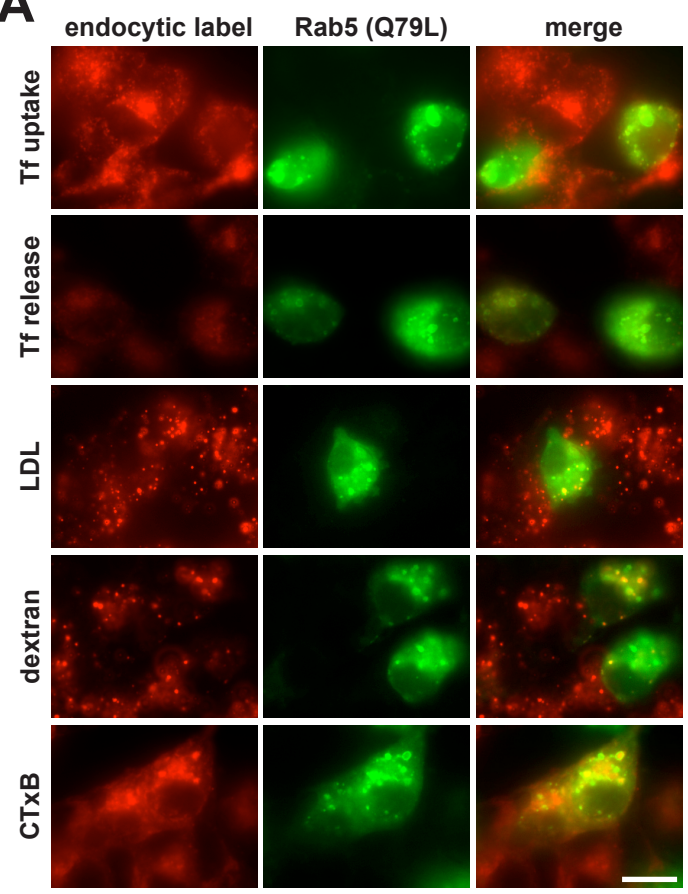

B

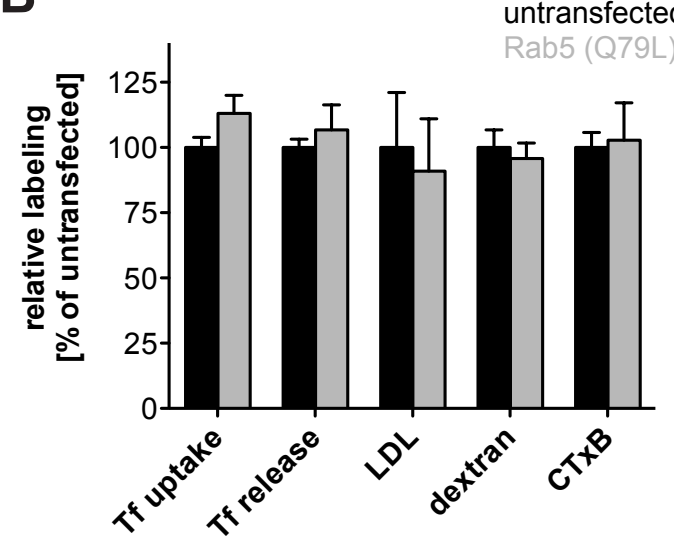

C
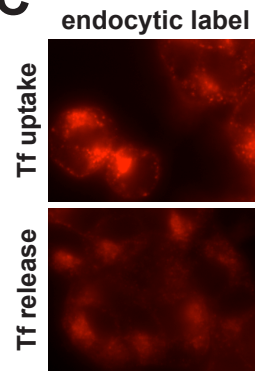

Rab5 (WT)

merge
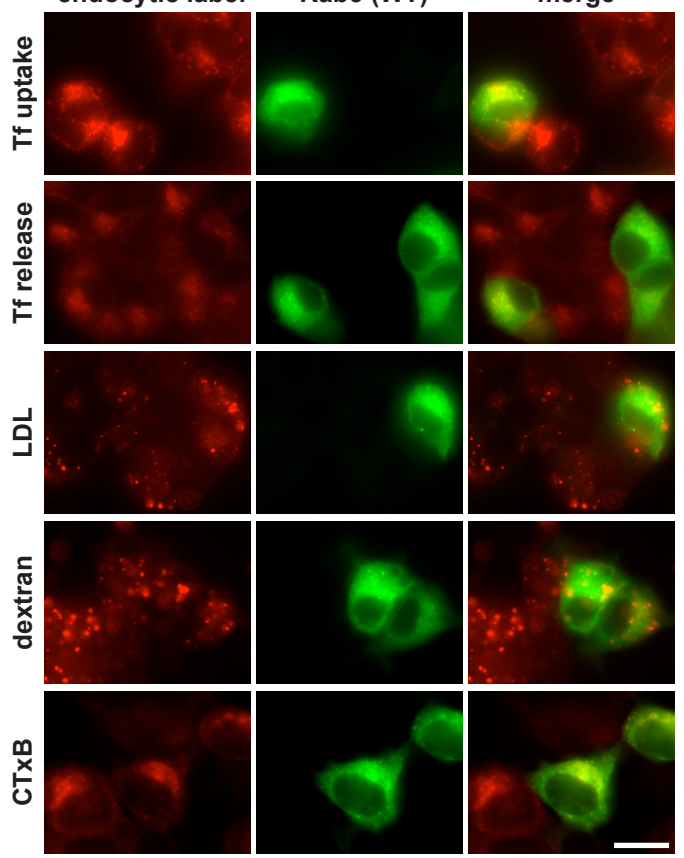

D
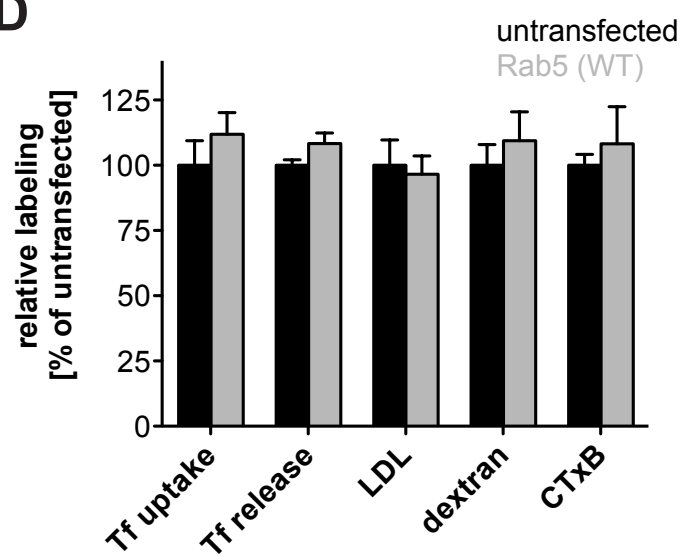

Figure 3-6: Expression of Rab5-GFP variants (wild-type or Q79L) does not affect endosomal trafficking in PC12 cells

(A) Typical images of PC12 cells showing both cells expressing dominant active (Q79L) Rab5-GFP (green) and control untransfected cells. Several endocytic labels were applied (each presented in red), as follows. For Transferrin (Tf) uptake, cells were incubated for 5 minutes at $37^{\circ} \mathrm{C}$ with 50 $\mu \mathrm{g} / \mathrm{ml} \mathrm{Tf}-A l e x a 594 ;$ for Tf release, cells were labeled in the same fashion, were briefly washed (twice) and were incubated at $37^{\circ} \mathrm{C}$ for 10 minutes in the absence of Tf. For low-density lipoprotein (LDL) uptake, cells were incubated for 5 minutes at $37^{\circ} \mathrm{C}$ with $5 \mu \mathrm{g} / \mathrm{ml} \mathrm{LDL}$-DiI. For dextran uptake, cells were incubated for 5 minutes at $37^{\circ} \mathrm{C}$ with $1 \mathrm{mg} / \mathrm{ml}$ dextran-Alexa594. For cholera toxin beta subunit (CTxB) uptake, cells were pre-incubated for 30 minutes on ice with 10

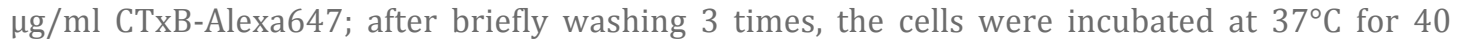
minutes, to allow the toxin to reach the Golgi. Scale $=15 \mu \mathrm{m}$.

(B) Quantification of the endocytic labeling. The red fluorescence (endocytic marker) of PC12 cells expressing dominant active (Q79L) Rab5-GFP was normalized to the fluorescence of untransfected cells. No significant differences could be detected ( $p>0.05$, t-tests). Quantification represents mean of 4 experiments \pm SEM.

(C-D) The same experiments as in A-B were repeated using the wild-type Rab5-GFP construct. Again, no significant differences could be detected between transfected and untransfected cells ( $p>0.05$, t-tests). Quantification represents mean of 4 experiments \pm SEM. 


\subsection{Endocytosed Vesicle Protein Reaching Bona Fide Endosomes}

\subsubsection{Dynamin-dependent transport of synaptic vesicle protein to endosomes}

The previous experiments established the fusion of a substantial fraction of RRP vesicles with endosomes. In order to follow the transit of vesicles through endosomes in a controlled fashion, the previously described experiments clearly relied on temporally defined snap shots of vesicle recycling after an externally applied stimulus. However, as the RRP vesicles are the ones mostly relied upon at physiological culture conditions, and as these vesicles seem to be the ones recycling through endosomes, one would expect to be able to see synaptic vesicles reaching endosomal compartments also in absence of such stimuli.

Even though it seems a coherent interpretation that the transient appearance of larger labeled organelles after an RRP releasing stimulus is due to endosomal fusion, the photo-oxidation and Qdot experiments could not clearly establish the molecular identity of early endosomes. The pHluorin experiments, on the other hand, suggested the involvement of early endosomal SNAREs in the recycling of RRP vesicles, but these experiments could not report fusion between vesicles and endosomes. Therefore, we decided to turn to a different fluorescent assay to test whether RRP vesicles can indeed reach bona fide endosomes.

We planned to visualize early endosomes by expressing GFP-tagged variants of the endosomal Rab5 in cell culture. To be able to clearly differentiate endosomes from synaptic vesicles (which also contain Rab5; Takamori et al., 2006), we wanted to use the dominant active (Q79L) Rab5 mutant, which causes formation of larger endosomes (Stenmark et al., 1994). To verify that the expression of the dominant active variant of Rab5 does not cause a general disturbance of endosomal trafficking, we first tested the construct in the neuroendocrine PC12 cell line and assayed the trafficking of various endocytic labels (Transferrin, low-density lipoprotein, dextran and cholera toxin beta subunit; Figure 3-6 A). When compared to untransfected PC12 cells, there were no differences in trafficking of any of these endocytic probes (Figure 3-6 A-B). In order to not rely solely on the expression of the dominant active Rab5-GFP, we intended to include experiments using wild-type Rab5-GFP (rendering the identification of endosomes based on size more difficult, but decreasing the likelihood of any disturbance of endosomal function). Therefore, we repeated the endosomal trafficking assay using the wild-type construct (Figure 3-6 C), and as expected, we could not detect any effects on endocytic trafficking when compared to trafficking in untransfected cells (Figure 3-6 C-D). 
A

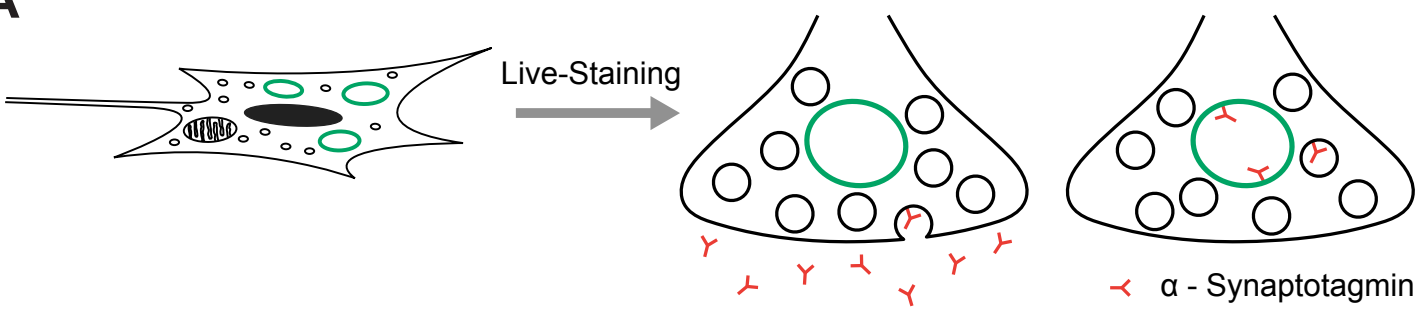

B
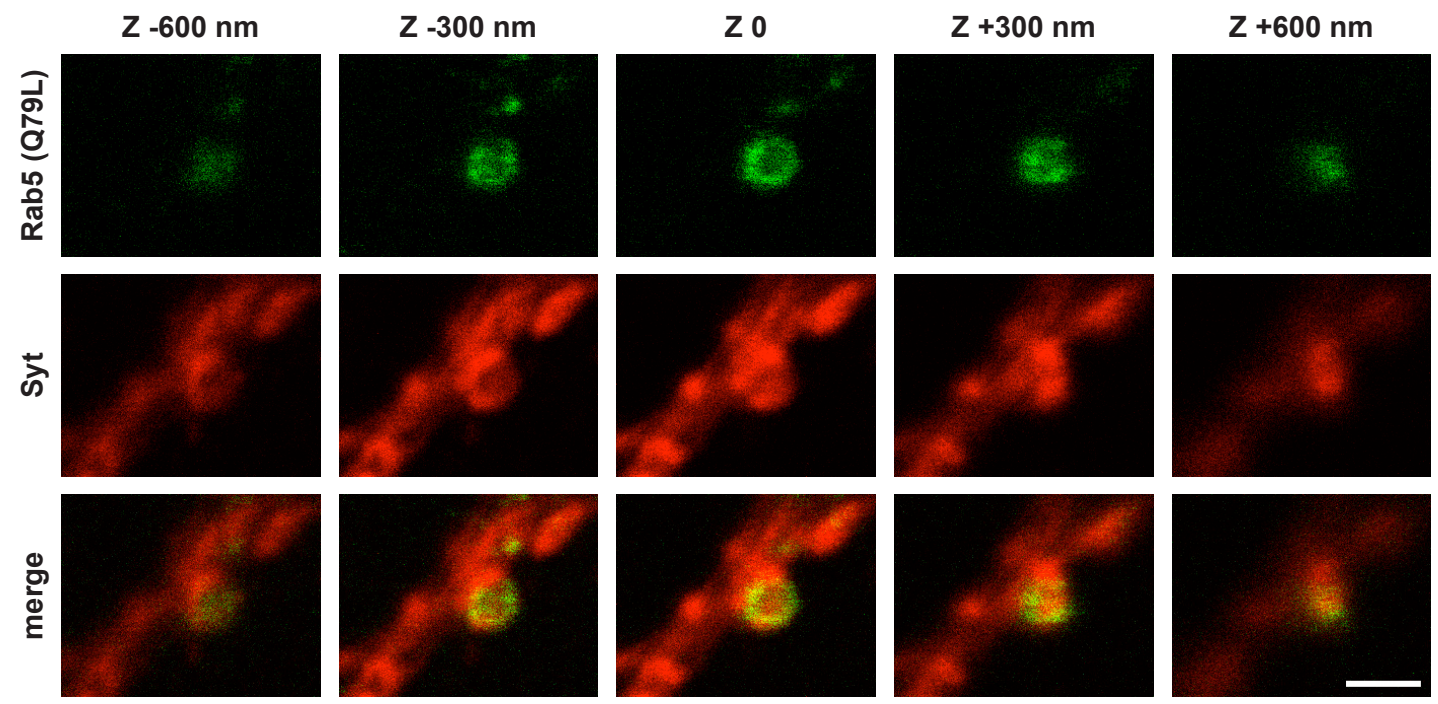

Figure 3-7: Synaptotagmin antibody uptake into endosomes

(A) Vesicle marker uptake into endosomes. Dominant active (Q79L) Rab5-GFP is expressed in hippocampal neurons. Cells are incubated with an antibody against the lumenal domain of Synaptotagmin (Table 2-1) for 5 minutes at $37^{\circ} \mathrm{C}$, to follow whether recently endocytosed Synaptotagmin molecules reach the endosome.

(B) Optical sectioning by confocal imaging indicates that the recently endocytosed vesicles (red) seem to reach an endosomal compartment (green), as shown by the overlap between the two. However, a proper quantitative analysis is difficult, as both the lateral and the axial resolution suffer from diffraction. Scale $=1.5 \mu \mathrm{m}$.

After having excluded any endosomal disturbances caused by the expression of our Rab5-GFP constructs, we returned to cultured hippocampal neurons and expressed the dominant active (Q79L) Rab5-GFP variant. We applied antibodies against the lumenal domain of Synaptotagmin (604.2; see Table 2-1) for 5 minutes at $37^{\circ} \mathrm{C}$ and acquired confocal Z-stacks of Rab5-GFP and Synaptotagmin signals (Figure 3-7). Although it appeared as if the Synaptotagmin antibodies colocalized with the endosomes, the limited lateral (in the $\mathrm{x}-\mathrm{y}$ plane; $\sim 250 \mathrm{~nm}$ ) and axial (along the z-axis; $\sim 500 \mathrm{~nm}$ ) resolution in conventional confocal microscopy prevented accurate colocalization analysis. We therefore resorted to a system of plastic embedding and thin sectioning (section 2.9.3) to drastically improve the attained axial resolution to about 80 $\mathrm{nm}$. In combination with STED imaging for the Synaptotagmin antibodies, this approach provides a three dimensional resolution of $70-80 \mathrm{~nm}$, sufficient to precisely assess 
colocalization of vesicles and endosomes. STED resolution for the imaging of endosomes is not necessary here, as their size is on the order of magnitude of the diffraction limit of light $(\sim 200 \mathrm{~nm})$, or even considerably bigger upon expression of the dominant active (Q79L) Rab5-GFP mutant (up to micrometer sizes).

A

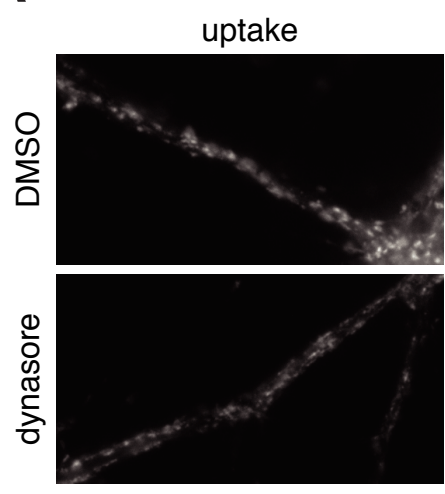

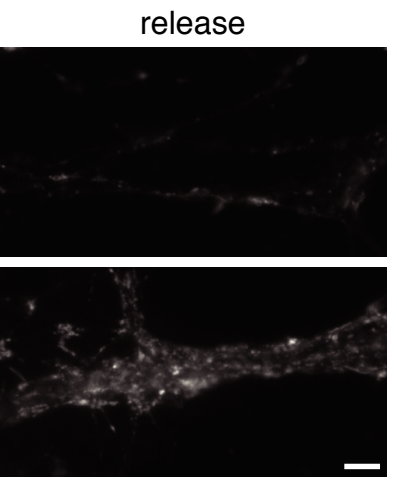

B

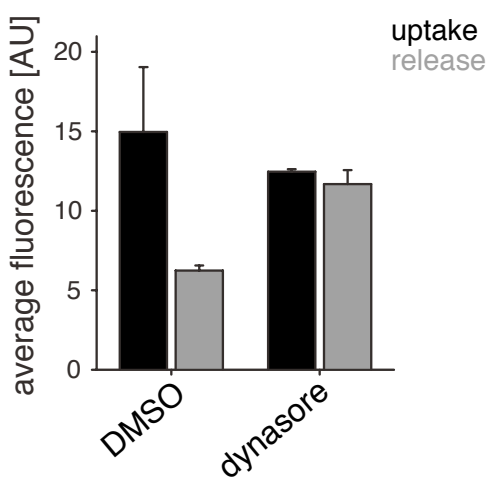

Figure 3-8: Dynasore inhibits synaptic vesicle recycling in hippocampal neurons

(A) FM uptake and release. Cultured hippocampal neurons were stimulated to recycle synaptic vesicles by application of $70 \mathrm{mM} \mathrm{KCl}$ in presence of the styryl dye FM 2-10. Upon endocytosis, the vesicles were labeled with the dye (top left panel); stimulating again in absence of the dye caused its release from the vesicles, resulting in a dimming of the synaptic puncta (top right panel). Dynasore pre-incubation (for 10 minutes at $37^{\circ} \mathrm{C}$ ) appeared to completely eradicate dye release (bottom panels), indicative of inhibited synaptic vesicle recycling (Newton et al., 2006). Control preparations were incubated with a corresponding amount of solvent (DMSO). Scale $=5 \mu \mathrm{m}$.

(B) Quantification of synaptic fluorescence. The fluorescence (above background) of the images was measured with a self-written routine in Matlab. The bars show mean \pm range of values of two independent experiments. Note that the formation of new fusion-competent vesicles (i.e. vesicle recycling) is completely blocked by dynasore.

Experiments were performed with the help of S. V. Barysch (STED Microscopy of Synaptic Function, European Neuroscience Institute Göttingen).

To have a means of assessing whether any observed colocalization of endosomes and synaptic vesicle marker is specific, we needed an inhibitor of endocytosis. The inhibitor should prevent the uptake of the anti-Synaptotagmin antibody into endocytosed synaptic vesicles, so that the antibodies would be also efficiently kept from reaching endosomes. The small molecule inhibitor dynasore blocks the small GTPase Dynamin (Newton et al., 2006), which is required for the final endocytic step of pinching-off of the vesicles (Sweitzer and Hinshaw, 1998). Therefore, dynasore promised to provide an efficient tool for our purposes, and we verified that it effectively inhibits endocytosis into releasable vesicles in our hands by assaying the uptake and release of FM 2-10 in hippocampal neurons (Figure 3-8). 

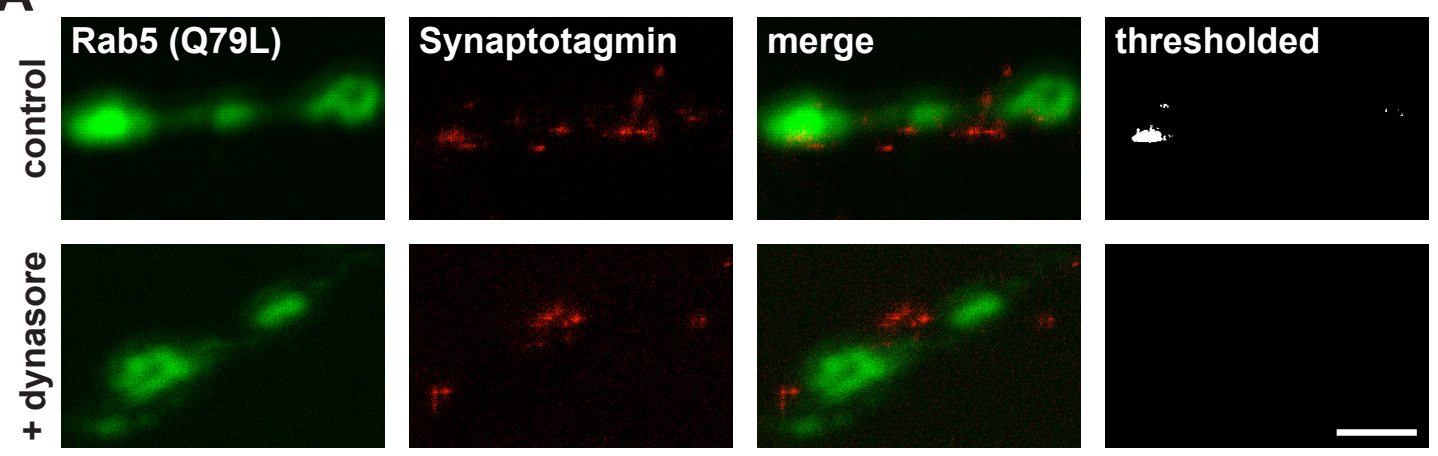

B

Rab5 (WT)
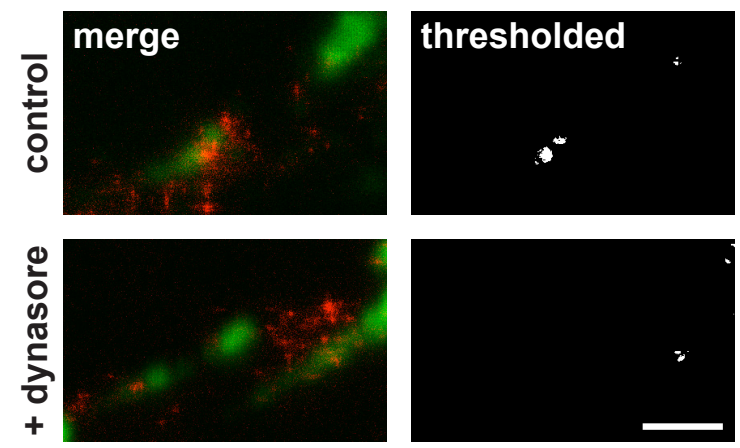

C

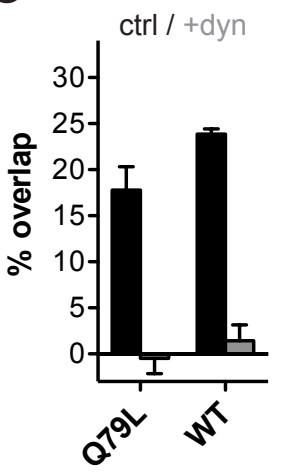

D

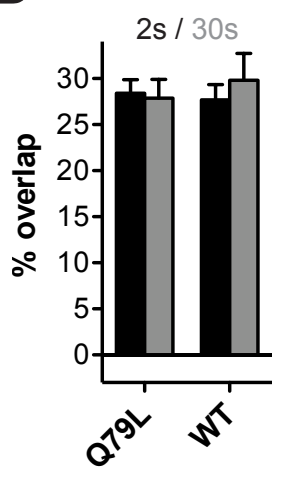

Figure 3-9: Analysis of overlap between synaptic vesicle marker and endosomes

(A-B) High resolution imaging of preparations expressing the dominant active (Q79L) Rab5-GFP (A) or wild-type Rab5-GFP (B). The vesicle marker (red) is imaged at a lateral resolution of $\sim 70$ $80 \mathrm{~nm}$ using STED microscopy, in preparations cut in ultrathin $(\sim 80 \mathrm{~nm})$ sections (see section 2.9.3); the Rab5-GFP (green) is imaged with confocal lateral resolution. Recently endocytosed vesicles (red) reach the endosomes (green) in control (top panels) but not in preparations treated with the Dynamin inhibitor dynasore (bottom panels). The area of overlap is highlighted in the rightmost panels (thresholded). Scale $=1 \mu \mathrm{m}$.

(C) Quantification of overlap for dominant active (Q79L) Rab5-GFP and wild-type Rab5-GFP. The fraction of the endosome area colocalizing with Synaptotagmin spots was determined and normalized to a maximum colocalization control (obtained from Synaptotagmin doubleimmunostaining, Opazo et al., 2010). Dynasore treatment significantly abolishes the overlap $(\mathrm{p}<0.01$, t-test). Data represent mean \pm SEM of 3 independent experiments. Overlap values were corrected by subtracting the overlap between the green signal and a mirror image of the red signal, which accounts for the negative values.

(D) Overlap of the synaptic vesicle marker with endosomes upon electrical stimulation. Experiments were performed as in A-C, after stimulating the samples using RRP-releasing protocols (20 Hz / 2 seconds) or recycling pool-releasing protocols (20 Hz / 30 seconds). The samples were then fixed and processed after 10 seconds of rest (as in Figure 3-4). Data analysis was as in C. Data represent mean \pm SEM of 4-6 experiments.

When we repeated the antibody-labeling experiment after plastic embedding / thin-sectioning and imaged the synaptic vesicle marker with STED microscopy, we again found the antibodies to reach the bona fide Rab5 positive endosomes (Figure 3-9 A, top panels). To test whether the observed colocalization is specific, we blocked clathrinmediated endocytosis by application of dynasore, which effectively prevented the Synaptotagmin antibodies from reaching the endosomes (Figure 3-9 A, bottom panels). When we repeated these experiments with the wild-type Rab5-GFP construct, we found 
no difference in the extent of colocalization when compared with the dominant active (Q79L) construct (Figure 3-9 B-C). We finally repeated these labeling experiments by applying defined electrical stimuli (Figure 3-9 D), rather than relying on the culture activity. We again observed substantial colocalization of endosomes and synaptic vesicle marker, when we applied an RRP releasing stimulus $(20 \mathrm{~Hz} / 2$ seconds; Figure 3-9 D, black bars). In line with the pHluorin experiments, stronger recycling pool releasing stimulation (20 Hz / 30 seconds) did not lead to an increase in the amount of colocalization (Figure 3-9 D, gray bars).

\subsubsection{Live-imaging of vesicle transit to endosomes}

To dynamically visualize the transit of synaptic vesicles to endosomes, we used videorate (28 frames per second) live-STED microscopy (see section 2.3.2.2) in the same preparation as above. Here, we again investigated whether strong stimulation $(20 \mathrm{~Hz} /$ 30 seconds; recycling pool releasing) would increase the amount of vesicles reaching the endosomes, which is unlikely in view of the pHluorin experiments and the previous experiments using electrical stimulation (Figure 3-9 D).

A
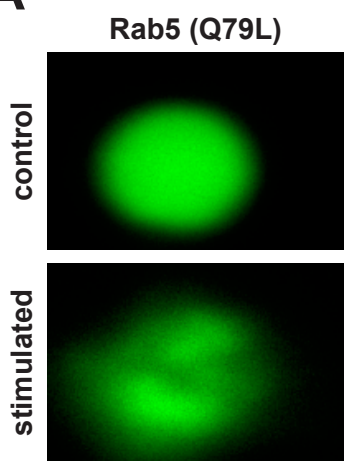

Synaptotagmin
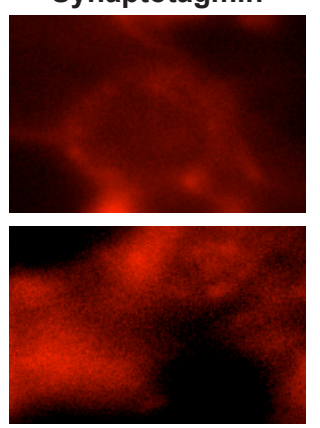

merge

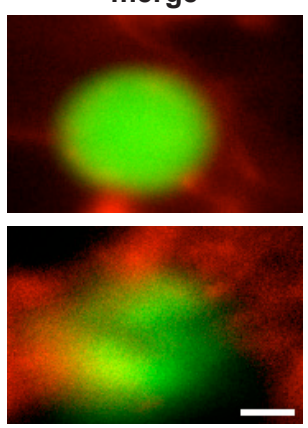

B

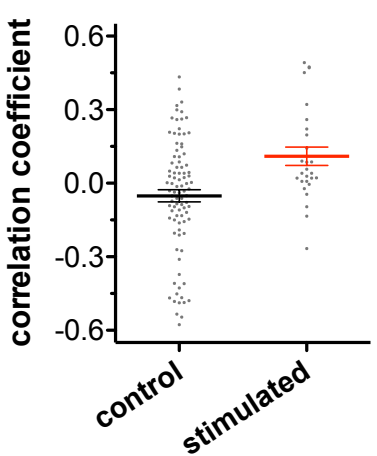

Figure 3-10: Live STED imaging of synaptic vesicles reaching endosomes

(A) Summed images from movies frames. Neurons expressing dominant active (Q79L) Rab5-GFP (green) were labeled with antibodies recognizing the lumenal domain of Synaptotagmin (red) (Westphal et al., 2008). Vesicles generally seem to avoid the endosome under resting conditions (top) and strong recycling pool stimulation (20 Hz / 30 seconds) only leads to a small increase in colocalization (bottom). Scale $=500 \mathrm{~nm}$.

(B) The correlation between dominant active (Q79L) Rab5-GFP and Synaptotagmin signals increases slightly upon prolonged stimulation $(20 \mathrm{~Hz} / 30$ seconds; $\mathrm{p}<0.05$, t-test). Data represent mean \pm SEM of 92 (non-stimulated) and 27 (stimulated) endosomal regions (from 63 and 18 movies, respectively). The scatter plots show individual values. 
In spite of a general avoidance of endosomal areas by the synaptic vesicles, we could observe synaptic vesicles reaching endosomes, both in preparations at rest and in stimulated preparations. We analyzed the movies by creating summed images of all movie frames (Figure 3-10 A) and by calculating the correlation between Rab5-GFP and Synaptotagmin signals. We found only a small increase in correlation upon strong stimulation (Figure 3-10 B), confirming again that it is predominantly the RRP that recycles through the endosome.

\subsection{Effects of Inhibiting Endosomal Function}

\subsubsection{Changes in vesicle pools}

As we found endosomal sorting to be of particular relevance for the RRP vesicles, we assumed that inhibition of endosomal sorting should result in readily observable effects on the RRP (and possibly on other vesicle pools as a consequence).

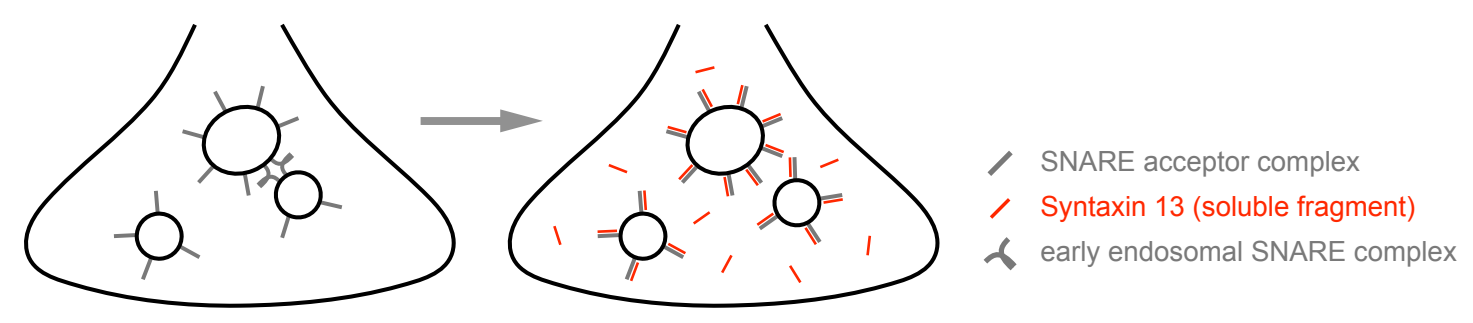

Figure 3-11: Blocking endosomal fusion by soluble Syntaxin 13 fragment

Soluble Syntaxin 13 fragments (red) are expressed in hippocampal neurons. They engage the endosomal SNAREs on the membranes of endosomes and synaptic vesicles, thus preventing formation of fusogenic SNARE complexes (Rizzoli et al., 2006). GFP was expressed together with (but not fused to) the Syntaxin 13 fragment by an IRES expression system, to identify transfected neurons. As a control, GFP was expressed alone.

To test this hypothesis, we expressed a soluble Syntaxin 13 fragment, designed to engage endosomal acceptor SNARE complexes in non-fusogenic complexes, thereby preventing synaptic vesicles from fusing with endosomes (Figure 3-11). We chose to target Syntaxin 13, as it is the only SNARE described to function exclusively in sorting endosomes (Prekeris et al., 1998; McBride et al., 1999). That this approach does not generally abolish endosome activity is shown by the fact that the recycling of several endocytic probes (Transferrin, low-density lipoprotein, dextran and cholera toxin beta subunit) was not affected in PC12 cells expressing the soluble Syntaxin 13 fragment (assayed as in Figure 3-6; personal communication, Ioanna Bethani, Max-Planck Institute for Biophysical Chemistry, Göttingen). 
A
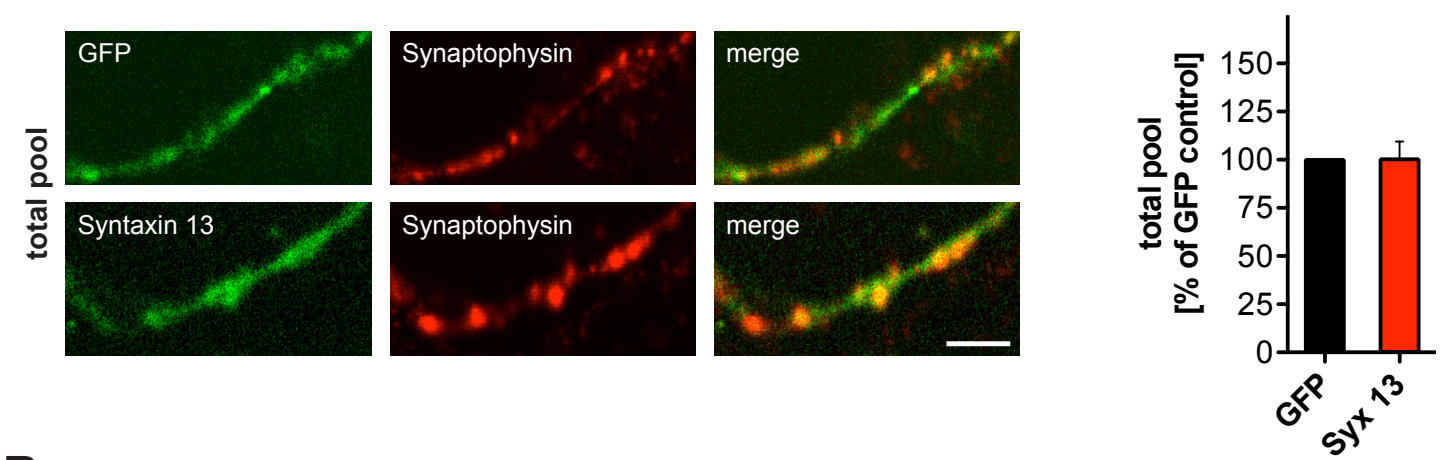

B
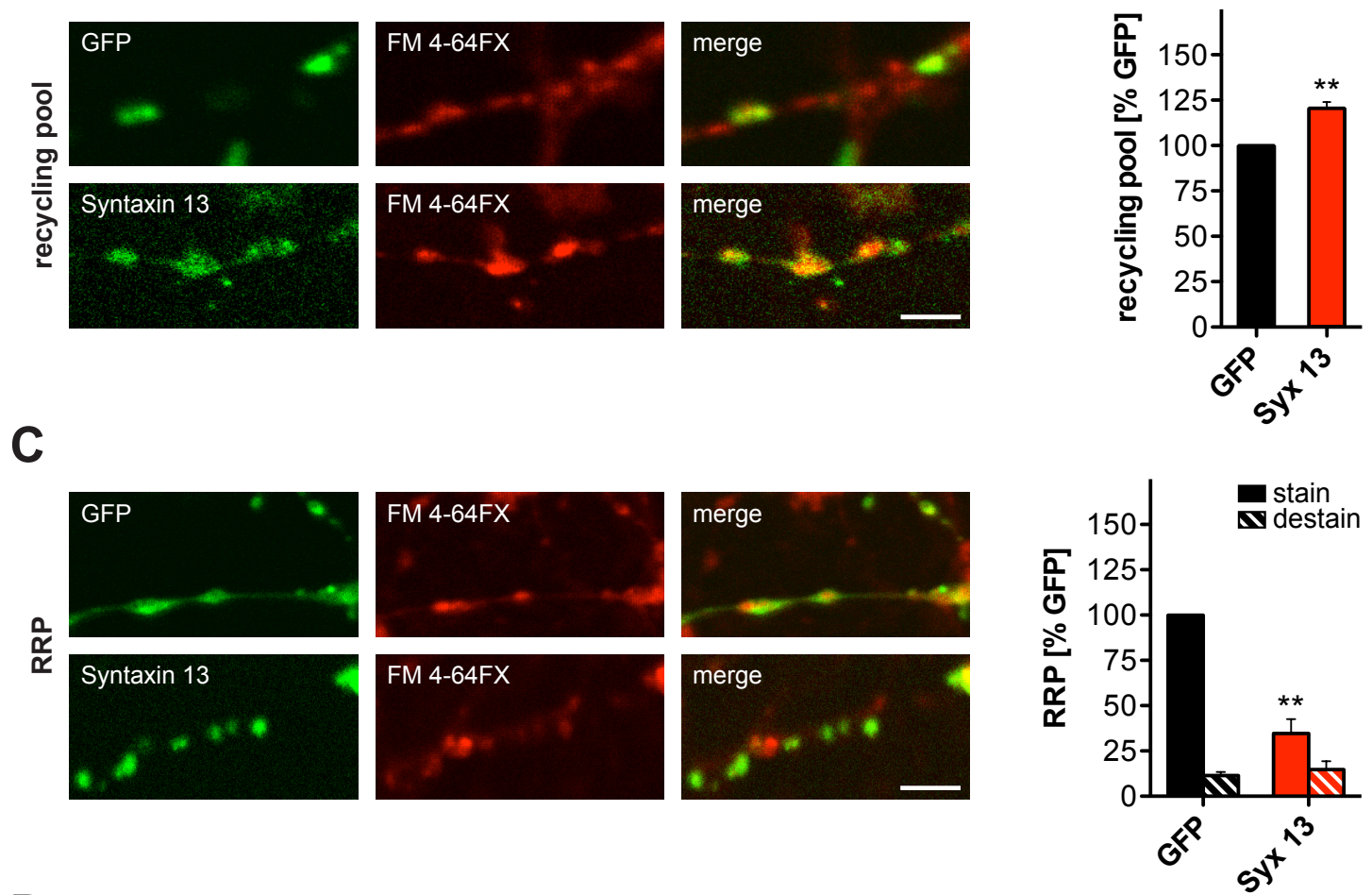

D
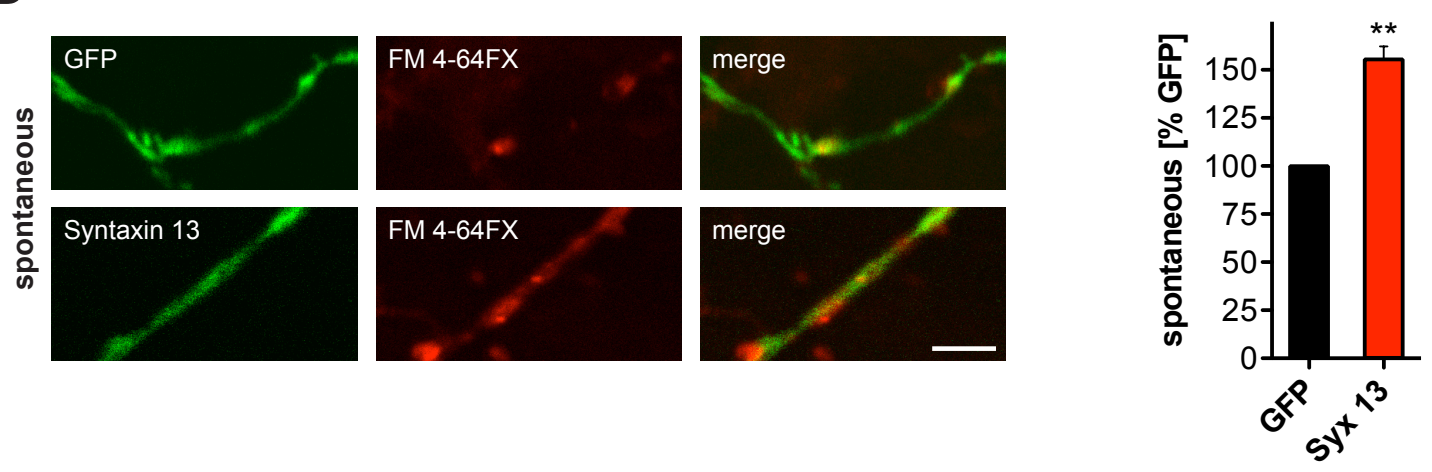

Figure 3-12: Effects of inhibiting endosomal fusion on synaptic vesicle pools

We expressed in hippocampal cultures soluble Syntaxin 13 fragment to block endosomal fusion. To identify the transfected cells, we co-expressed GFP using an IRES system; GFP alone was expressed in control preparations, to control for possible transfection effects.

(A) The total vesicle pool visualized by immunostaining for Synaptophysin (red). The top panels indicate axons expressing GFP alone; the lower panels show axons expressing both GFP and the Syntaxin 13 fragment. Quantification of the total vesicle pool (bar graph), expressed as percent of the GFP-only control, shows no effect based on the inhibition of endosomal fusion. (legend continued on next page) 
(B) The recycling pool was labeled with FM 4-64FX (red), by stimulating at $20 \mathrm{~Hz}$ for 30 seconds. Quantification of the recycling pool (bar graph), expressed as percent of the GFP-only control, shows a $\sim 20 \%$ increase in the recycling pool upon blocking of endosomal fusion $(\mathrm{p}<0.01, \mathrm{t}$-test). (C) The RRP was labeled with FM 4-64FX (red), by stimulating at $20 \mathrm{~Hz}$ for 2 seconds. Quantification of the RRP (bar graph), expressed as percent of the GFP-only control, shows a $\sim 60 \%$ decrease in the RRP upon blocking of endosomal fusion ( $p<0.01$, t-test). Note that the FMloaded RRP vesicles could be re-released by a $20 \mathrm{~Hz} / 30$ second stimulus in both conditions ("destain", hashed bars).

(D) The spontaneously recycling vesicles were labeled by incubation with FM 4-64FX for 15 minutes in presence of $1 \mu \mathrm{M}$ tetrodotoxin (to block action potential evoked vesicle release). Quantification of the spontaneous pool (bar graph), expressed as percent of the GFP-only control, shows a $\sim 50 \%$ increase in the spontaneous pool upon blocking of endosomal fusion $(\mathrm{p}<0.01, \mathrm{t}$ test).

All scales $=2.5 \mu \mathrm{m}$. All data are presented as mean \pm SEM of 3 independent experiments.

In order to identify the neurons expressing the soluble Syntaxin 13 fragment, we co-expressed GFP, used an internal ribosomal entry site (IRES) system (see section 2.10.1) to avoid any effects related to tagging the fragment with GFP. To test whether transfection / expression itself causes any perturbations, we expressed GFP alone in control experiments.

Blocking endosomal fusion by expression of soluble Syntaxin 13 fragment did not reduce the total synaptic vesicle pool, when compared with the GFP control (Figure 3-12 A). This implies that biogenesis and transport of synaptic vesicles to the synapses are not affected and thus do not rely on vesicle-to-endosome fusion. When labeling the RRP with FM 4-64FX (20 Hz / 2 seconds stimulation), we observed a strong decrease in RRP size, in comparison to the GFP control ( $\sim 60 \%$ reduction; Figure 3-12 C). That the labeled vesicles are, however, functionally recycling synaptic vesicles, is shown by their ability to release upon a second stimulation train $(20 \mathrm{~Hz} / 30$ seconds; destain; Figure 3-12 C). Interestingly, we could also observe a $20 \%$ increase in the recycling pool size (Figure 3-12 B) and a 50\% increase in spontaneous release upon endosomal inhibition (Figure 3-12 D). As our results so far have indicated no dependence of the recycling pool on endosomes, these effects might constitute a consequence of the drastic effect on the RRP vesicles, rather than a direct influence of endosomal inhibition on these vesicles.

To verify that the observed effects on the RRP, recycling pool and spontaneous pool are truly based on inhibition of the formation of fusogenic SNARE complexes, we repeated the same experiments with a soluble Syntaxin 13 fragment also lacking the SNARE domain (aa 1-186; Figure 3-13). Expression of these Syntaxin 13 fragments did not show any effects on vesicle pools, supporting the specificity of our approach to block endosomal fusion. 
A

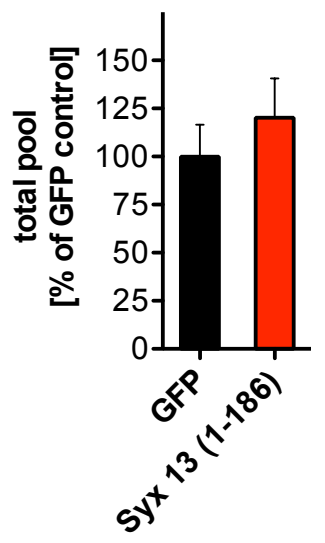

B

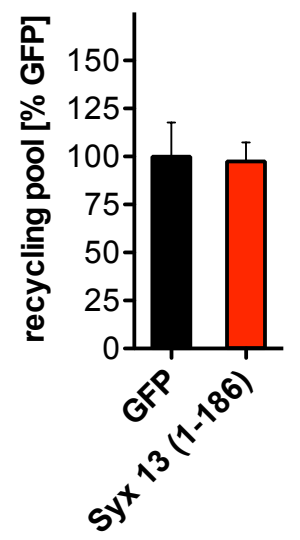

C

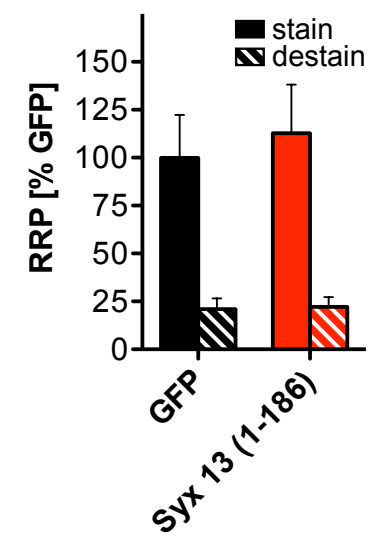

D

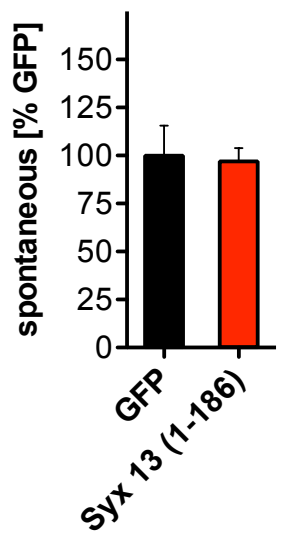

Figure 3-13: Quantification of vesicle pools upon expression of soluble Syntaxin 13 fragment lacking the SNARE domain

Experiments and data analysis were performed as in Figure 3-12, only that we used soluble Syntaxin 13 fragments lacking the SNARE domain (aa 1-186). All pools are expressed as percent of the GFP-only control. The total pool (A) was quantified from Synaptophysin immunostaining. The recycling pool (B) and the RRP (C) were labeled with FM 4-64FX, by stimulating at $20 \mathrm{~Hz}$ for 30 and 2 seconds, respectively. Note that the FM-loaded RRP vesicles could be re-released by a $20 \mathrm{~Hz} / 30$ second stimulus in both conditions ("destain", hashed bars). Spontaneously recycling vesicles (D) were labeled by incubation with FM 4-64FX for 15 minutes in presence of $1 \mu \mathrm{M}$ tetrodotoxin. Note that expression of Syntaxin 13 fragments lacking the SNARE domain does not exhibit any vesicle pool related effects. Data are presented as mean \pm SEM of 2-4 independent experiments.

\subsubsection{Monte Carlo modeling of RRP release}

The observation that the RRP is strongly reduced upon blocking of endosomal fusion confirms our hypothesis that the RRP requires endosomal sorting for its proper function. As the total number of synaptic vesicles is not affected in absence of endosomal fusion (Figure 3-12 A), it remains to be solved what could account for the observed decrease in the response to an RRP-stimulus (Figure 3-12 C).

We assumed that fused RRP vesicles are occasionally retrieved as "dirty" vesicles (impurified with e.g. plasma membrane material) that would normally get sorted upon fusion with an endosome. If endosomal function is inhibited (as is the case upon expression of the soluble Syntaxin 13 fragment), these "dirty" vesicles might remain non-sorted and exhibit lower fusion-competence, and thus release probability, than normal RRP vesicles. To obtain an estimate of the release efficiency of "dirty" RRP vesicles that would explain the experimentally observed $\sim 60 \%$ decrease in RRP size upon endosomal inhibition, we generated a Monte Carlo model constructed from vesicle numbers, release rates and endocytosis rates as previously published or determined in this study (Figure 3-14 A; see section 2.10.2 for a detailed description of the model). The model first monitored the normal release to a 20 action potential stimulus, followed by a 30 second recovery period. Afterwards, endosomal sorting was blocked and "dirty" 
vesicles were allowed to accumulate during a period of prolonged activity (1000 action potentials at $1 \mathrm{~Hz}$ ). Finally, the response to a second 20 action potential stimulus was measured and expressed relative to the first one (color scale in Figure 3-14 B). Multiple models simulated the RRP release in dependence on the stimulation frequency and varying values for the variable of interest: the release probability of the "dirty" vesicles. The model suggests that the observed decrease of the RRP at $20 \mathrm{~Hz}$ stimulation (Figure 3-12 C) can be explained by an approximately 6-fold reduction in release probability of "dirty" vesicles compared to normal RRP vesicles (Figure 3-14 B, circle).

This model once more highlights the importance of endosomal sorting for RRP function: (1) absence of endosomal sorting decreases the release probability of RRP vesicles by a factor of 6; and (2) even at stimulation frequencies of only $0.5 \mathrm{~Hz}$, such a 6fold decrease in release probability would already lead to a $\sim 40 \%$ decrease in RRP response (Figure 3-14 B, arrowhead).

A

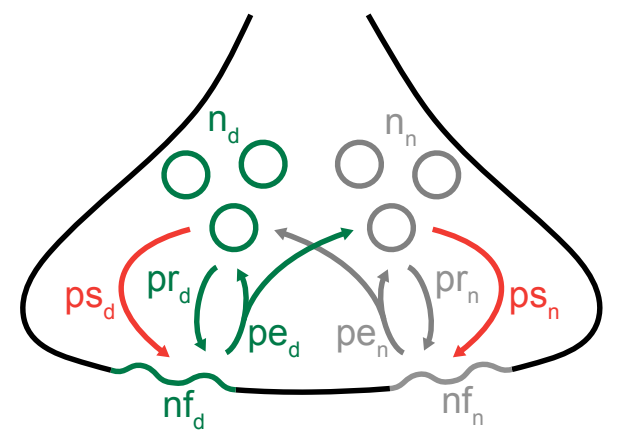

B

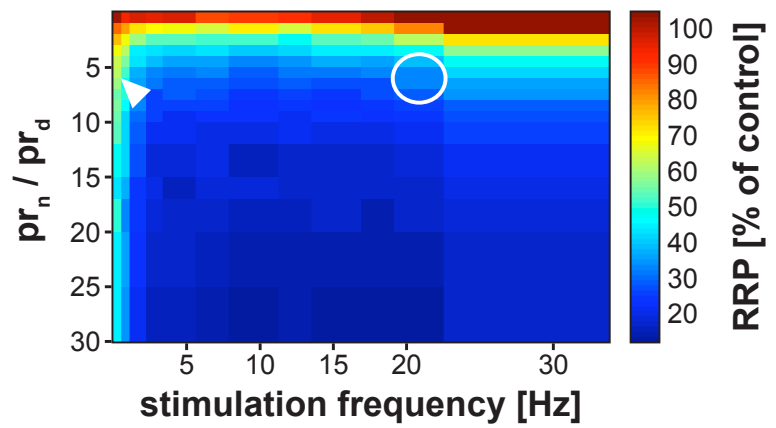

Figure 3-14: Monte Carlo modeling of vesicle pools in absence of endosomal sorting (A) Monte Carlo model of RRP recycling. Normal $(\mathrm{n})$ vesicles were placed in the synapse; endocytosis occasionally retrieved "dirty" (d) vesicles. The vesicle numbers (n), the number of fused vesicles (nf), their probabilities to release upon stimulation (pr) or spontaneously (ps), their probabilities to be endocytosed (pe) and the probability that a normal vesicle is retrieved as a "dirty" one (and vice-versa) were all modeled according to our own data or to values obtained from the literature (see section 2.10.2 for details). The response to a 20 action potential train is monitored first in the absence of any "dirty" vesicles. This value is then compared to the response to an identical stimulus applied after allowing the accumulation of "dirty" vesicles during a 1000AP, $1 \mathrm{~Hz}$ stimulation train (with the endosomal sorting inactive).

(B) The RRP release upon the second 20 action potential train (inactive endosomal sorting), expressed as percentage of the first train (control), in dependence on the stimulation frequency (X-axis) and the relative probability of release of "dirty" vesicles (Y-axis). The experimental results from Figure 3-12 ( $\sim 60 \%$ reduction in RRP response) are best explained by a model in which the "dirty" vesicles exhibit a 6-fold lower release probability than the normal ones (circle). Note that according to this model, even at stimulation frequencies of only $0.5 \mathrm{~Hz}$, such a 6 -fold drop in release probability would already cause a reduction of $\sim 40 \%$ in the size of the RRP response (arrowhead). 


\subsection{The Recycling-Step Necessitating Endosomal Sorting}

Having established the requirement of endosomal sorting for RRP vesicles, the question of which step in vesicle recycling calls for the involvement of the endosome remains unanswered. Is endosomal sorting required because of vesicles intermixing with the plasma membrane upon exocytosis, or does endocytosis retrieve contaminating molecules from the plasma membrane together with the vesicle patches?

A
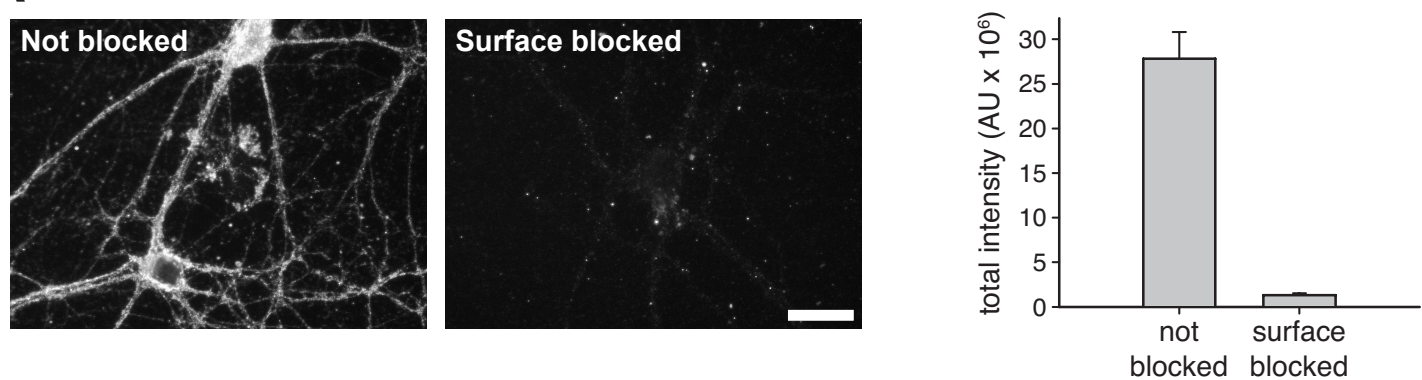

B
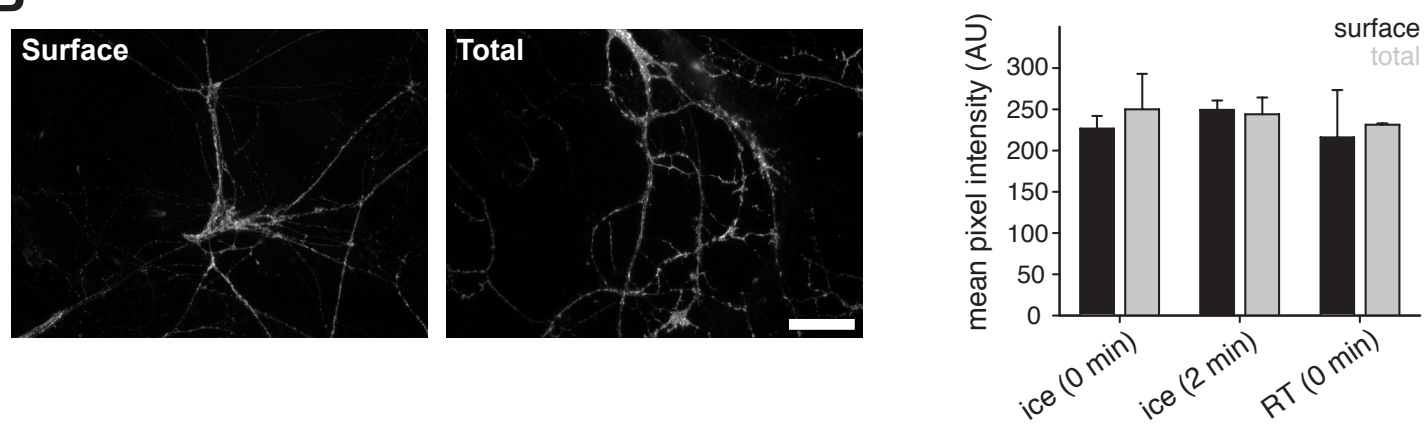

Figure 3-15: Blocking of surface Synaptotagmin epitopes together with inhibition of endocytosis allows for specific investigation of recently exocytosed vesicles

(A) Blocking pre-existing Synaptotagmin surface epitopes before stimulating exocytosis. Hippocampal neurons were stained with fluorescently-coupled antibodies against the lumenal domain of Synaptotagmin (604.2-Oyster550; see Table 2-1) either without (left panel) or with (middle panel) a preceding treatment with the same unlabeled anti-Synaptotagmin antibodies. This treatment was effective in blocking surface epitopes, as it drastically reduced the total fluorescence intensity (bar graph). Scale $=25 \mu \mathrm{m}$.

(B) Endocytosis block to avoid investigation of re-internalized vesicles. Endocytosis of recently fused vesicles needed to be prevented, as it would have resulted in the analysis of endocytosed Synaptotagmin-labeled vesicles, instead of plasma membrane-exposed material. Therefore, the labeling was performed either at $4{ }^{\circ} \mathrm{C}$ or in absence of $\mathrm{Mg}^{2+}$ and $\mathrm{Ca}^{2+}$ at room temperature, to allow for exocytosis but not endocytosis. To test the effectivity of this endocytosis block, we labeled hippocampal neurons with unlabeled antibodies against the lumenal domain of Synaptotagmin (604.2; see Table 2-1), fixed them (immediately after a brief (ice-cold) wash or with an additional 2 minutes incubation on ice) and stained them with secondary fluorescent antibodies either without (left panel; surface) or with (middle panel; total) permeabilization. The fluorescence intensity quantification revealed no difference between permeabilized and nonpermeabilized samples, underscoring that the chosen labeling conditions result in all Synaptotagmin antibodies persisting at the surface (i.e. block of endocytosis), even after a short incubation of the preparations after labeling. Scale $=25 \mu \mathrm{m}$. 


\subsubsection{Exocytosis maintains synaptic vesicle identity}

The current literature is ambiguous about whether synaptic vesicle molecules separate in the plasma membrane upon exocytosis. While Synaptotagmin has been found to remain clustered in the plasma membrane (Willig et al., 2006), when investigated by antibody labeling and STED microscopy, various pHluorin-based experiments suggest mixing of components among fused vesicles (see discussion in Fernandez-Alfonso and Ryan, 2006). To investigate this question further, we used 2-color STED microscopy, allowing us to simultaneously follow exocytosis of two synaptic vesicle proteins and to measure their colocalization in the plasma membrane.

To focus solely on the step of exocytosis, we needed to avoid analysis of synaptic vesicle protein clusters that are stranded in the plasma membrane (Gandhi and Stevens, 2003; Wienisch and Klingauf, 2006), which could be achieved by pre-blocking the surface-exposed Synaptotagmin molecules with an unlabeled antibody against the lumenal domain of Synaptotagmin (604.2, see Table 2-1). The efficiency of this treatment was validated by performing immunostainings on neuronal cultures either with or without pre-blocking of Synaptotagmin epitopes (Figure 3-15 A).

After pre-blocking of Synaptotagmin epitopes, we allowed exocytosis to deliver new synaptic vesicles to the plasma membrane. In order to ensure exclusive investigation of synaptic vesicle clusters present in the plasma membrane, as opposed to already internalized vesicles, we performed the experiments either on ice or in absence of the divalent ions $\mathrm{Mg}^{2+}$ and $\mathrm{Ca}^{2+}$. Both treatments effectively inhibit endocytosis, as shown by immunostaining experiments with and without permeabilization (Figure 3-15 B), and thus enable us to only investigate synaptic vesicle material in the plasma membrane.

When we measured the colocalization of Synaptotagmin and Synaptophysin in the plasma membrane upon exocytosis by using 2-color STED microscopy, we were unable to detect a difference compared to a positive colocalization control (Figure 3-16 A-B). This finding was independent of the method of inhibition of endocytosis employed (ice or no divalents; Figure 3-16 C-D). Additionally, measurements of the spot-size of Synaptotagmin and Synaptophysin puncta revealed that both proteins form small clusters of at most $\sim 60-70 \mathrm{~nm}$ (Figure 3-16 E). These findings suggest that at least two of the most prominent synaptic vesicle components (Synaptotagmin and Synaptophysin; Takamori et al., 2006) remain co-clustered upon exocytosis. Therefore, the synaptic vesicle components do not seem to intermix with the plasma membrane upon exocytosis, arguing that exocytosis per se does not necessitate endosomal sorting. 
A
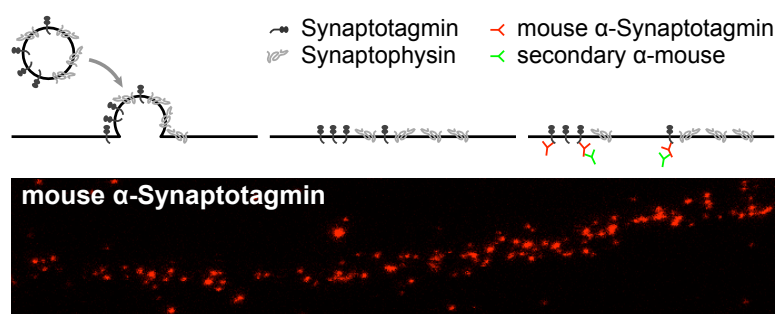

secondary a-mouse
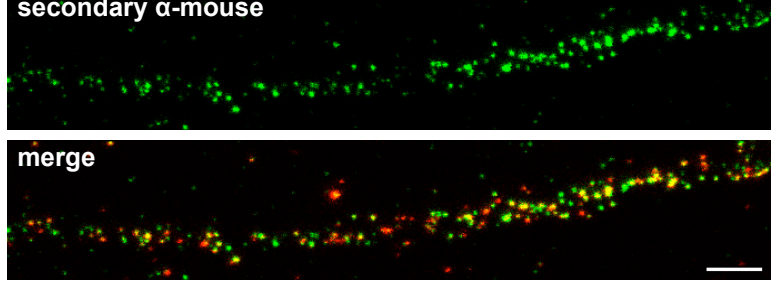

\section{B}
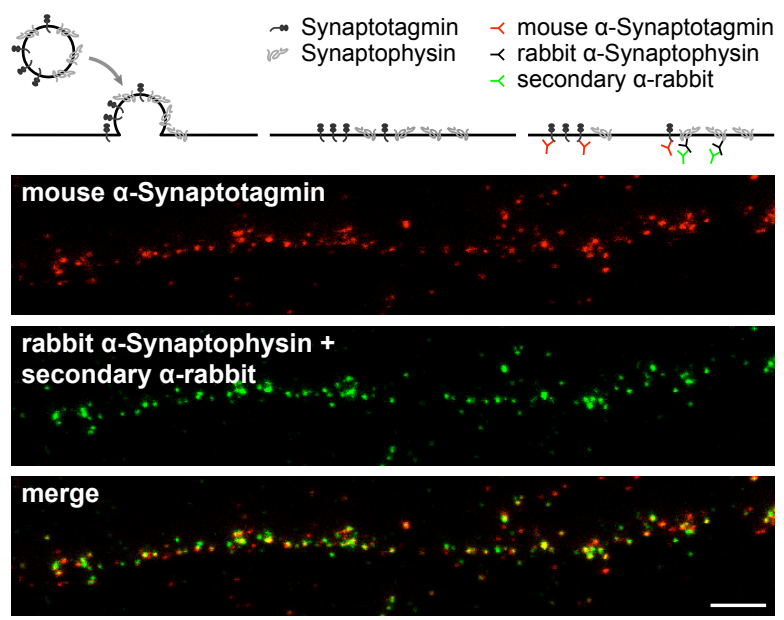

C

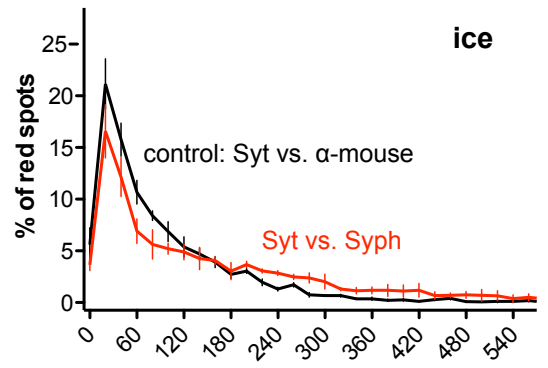

distance between red and green spots [nm]

D

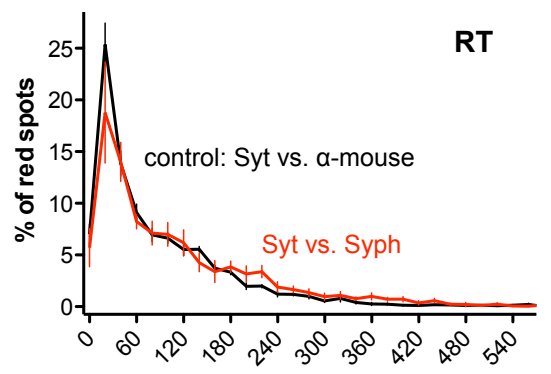

distance between red and green spots [nm]

E

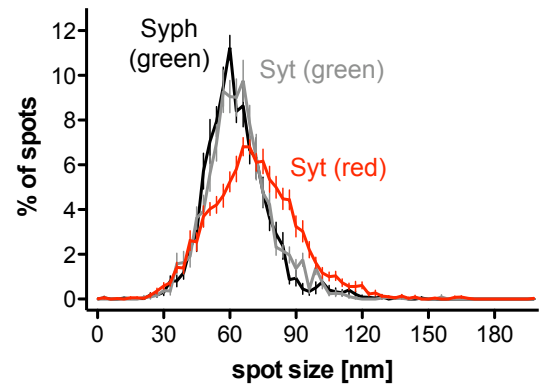

Figure 3-16: Synaptotagmin and Synaptophysin remain co-clustered upon exocytosis

(A) Colocalization positive control. Only recently exocytosed vesicles are investigated, with the experiments performed after blocking the surface Synaptotagmin epitopes with nonfluorescently coupled antibodies (not shown in the cartoon; see Figure 3-15 A). Neurons are then incubated with Atto 647N-coupled mouse Synaptotagmin antibodies (red; 604.2, see Table 2-1). Endocytosis is blocked either by keeping the neurons on ice, or by incubating at room temperature in absence of divalents (see Figure 3-15 B), to prevent the internalization of the label. After fixation, the samples are immunostained with secondary anti-mouse antibodies coupled to Atto 590 (green), resulting in Synaptotagmin being double-labeled in red and green. Note the high colocalization in 2-color STED microscopy. Scale $=1 \mu \mathrm{m}$.

(B) Synaptotagmin/Synaptophysin colocalization. Neurons are incubated with Atto 647Ncoupled mouse Synaptotagmin antibodies (red, as in A) and rabbit antibodies that recognize the lumenal domain of Synaptophysin (G96, see Table 2-1). After fixation, the samples are immunostained with secondary anti-rabbit antibodies (Atto 590, green) to visualize the Synaptophysin staining. Samples were treated exactly as in A. Scale $=1 \mu \mathrm{m}$.

(C-D) Spot-to-spot distance analysis for experiments where endocytosis was blocked by low temperature, on ice (C) or at room temperature in absence of divalents (D). Note that the two sets of distributions are very similar.

(E) Spot size. Both Synaptotagmin and Synaptophysin form well-defined clusters of about 60-70 $\mathrm{nm}$, within the resolution of the microscope - the real size of the clusters being thus at these values or lower.

All data (C-E) represent mean \pm SEM of 3-6 independent experiments. 


\subsubsection{Endocytosis leads to impurities in synaptic vesicle composition}

As synaptic vesicle proteins seemed to remain perfectly clustered upon exocytosis, we wanted to investigate whether endocytosis leads to uptake of "dirty" vesicles by retrieval of plasma membrane material along with the vesicle components (as we assumed for the Monte Carlo simulations; see section 3.4.2).

In a previously performed set of experiments, we used immunostaining of hippocampus neurons after thin sectioning (Punge et al., 2008) to investigate the colocalization of Synaptophysin and the plasma membrane SNAREs Syntaxin 1 or SNAP25 inside the boutons either before or after stimulation. We found an increase in colocalization upon stimulation $(70 \mathrm{mM} \mathrm{KCl})$, suggesting that endocytosis results in uptake of the plasma membrane proteins Syntaxin 1 and SNAP-25 into the synaptic vesicle cluster (Hoopmann, 2008). However, as these experiments could not exclude the possibility that the plasma membrane material is endocytosed by a bulk endocytosis mechanism, we devised a different assay to investigate the molecular composition of small endocytosed vesicles more directly.

A

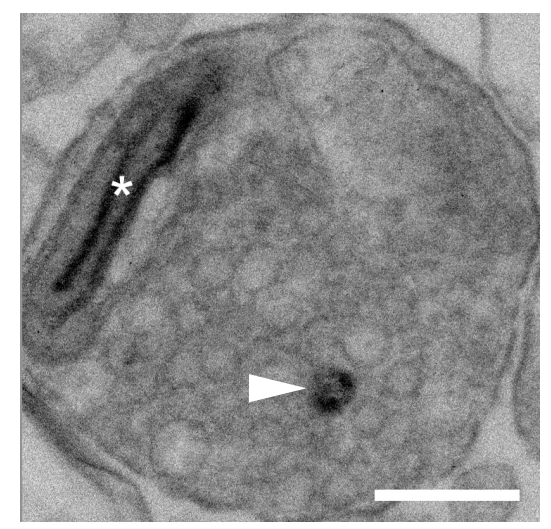

B

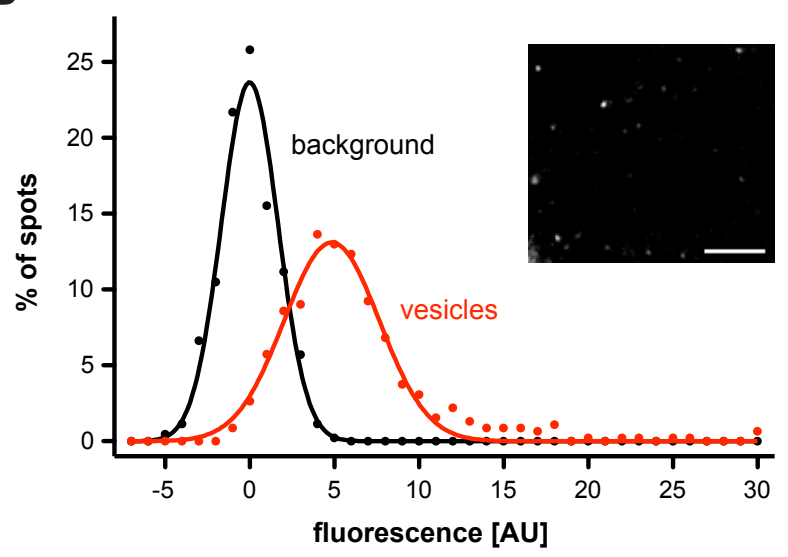

Figure 3-17: Labeling of small vesicles by endocytosis in synaptosomes

(A) Synaptosomal preparations were labeled with FM 1-43 (using $50 \mathrm{mM} \mathrm{KCl}$ stimulation) and the electron dense diaminobenzidine precipitate was generated by photo-oxidation (as in Figure 3-4). The micrograph shows one labeled vesicle (arrowhead). Note that the large dark structure marked by an asterisk (top left corner) is a mitochondrion (labeled by diaminobenzidine independent of the FM 1-43 presence; Grabenbauer et al., 2005). Scale $=200 \mathrm{~nm}$.

(B) Intensity histograms of FM-labeled vesicles adsorbed onto glass coverslips. Background intensity (black) follows an almost perfect Gaussian fit with a peak around zero fluorescence. The intensities of the vesicles (red) are also normally distributed. Data points show the actual distribution, line plots are Gaussian fits. The inset shows an example image of labeled vesicles (the image was smoothed for display, as the signal-to-noise ratio is fairly low). Scale $=5 \mu \mathrm{m}$.

We conclude that the vesicle labeling is normally distributed, indicating a relatively homogeneous pool of small (panel A) vesicles, with few larger organelles, which would necessarily appear brighter, as FM fluorescence is directly proportional to the surface of the labeled membrane. 


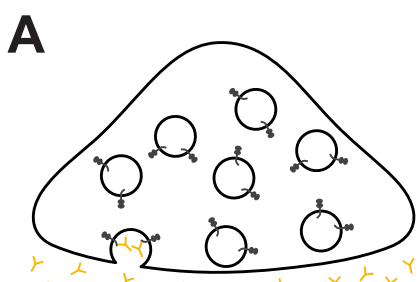

$\sim$ Synaptotagmin

B

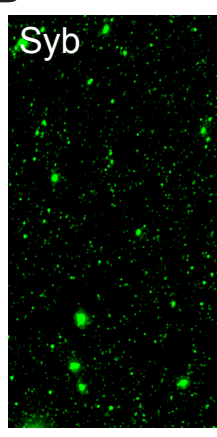

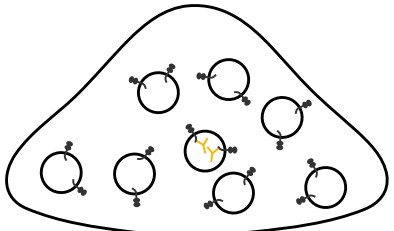

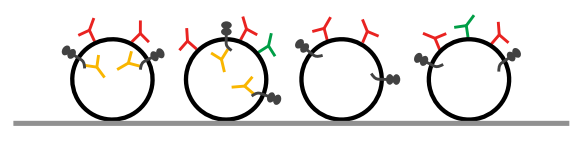

$\alpha$ - Synaptotagmin (lumenal domain)

$\prec \alpha$ - SV-marker

$\prec a$ - Protein-of-interest

C

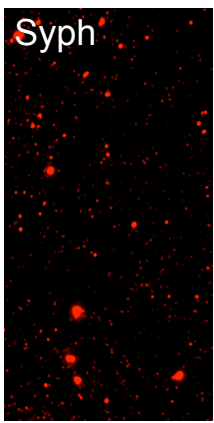

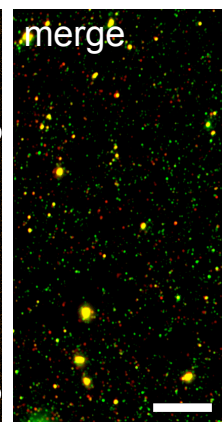

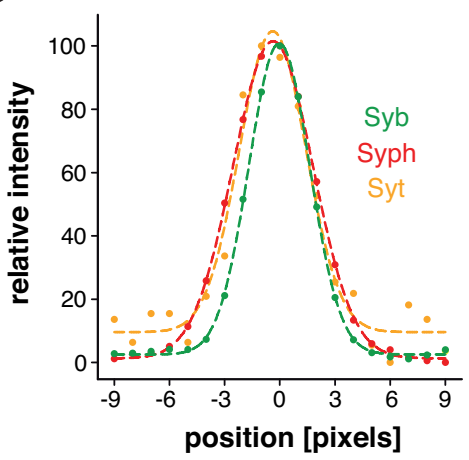

Figure 3-18: In vitro immunoassay to study recently endocytosed vesicle composition

(A) Synaptosomes were purified and stimulated by depolarization $(50 \mathrm{mM} \mathrm{KCl})$ in presence of fluorescently-coupled antibodies against the lumenal domain of Synaptotagmin (orange; 604.2Oyster550, see Table 2-1). The vesicles were isolated, adsorbed onto glass coverslips, and immunostained for a synaptic vesicle marker (red) and a protein of interest (green).

(B) Typical images of vesicles immunostained for Synaptobrevin (Syb) as protein of interest and Synaptophysin (Syph) as synaptic vesicle marker. Several recently endocytosed vesicles are indicated by circles in the (dim) Synaptotagmin (Syt) channel. Scale $=7.5 \mu \mathrm{m}$.

(C) Example of line-scans through a spot (vesicle) colocalizing in all three channels. Data points show raw data (normalized to 100 arbitrary units), dashed lines are Gaussian fits. Colocalization was assessed based on the correlation coefficient between the different signals.

To this end, we wanted to turn to rat brain synaptosomes (see section 2.5.1), allowing for the isolation of individual synaptic vesicles after fluorescence labeling. We first depolarized purified synaptosomes $(50 \mathrm{mM} \mathrm{KCl})$ in presence of the styryl dye FM 143 , to label recently endocytosed synaptic vesicles. When we investigated the labeled synaptosomes by photo-oxidation electron microscopy (as in Figure 3-4), we found the labeled vesicles to be small in diameter and easily distinguishable from unlabeled vesicles (Figure 3-17 A). Furthermore, when we isolated synaptic vesicles from labeled synaptosomes, the labeled vesicles appeared homogeneous in fluorescence intensity, suggesting homogeneity in size (Figure 3-17 B). These observations argue that bulk endocytosis events must be rather rare in our preparations, as the larger invaginations would provide a much bigger membrane area for FM-dye binding and would thus exhibit much higher fluorescence intensity. 
A

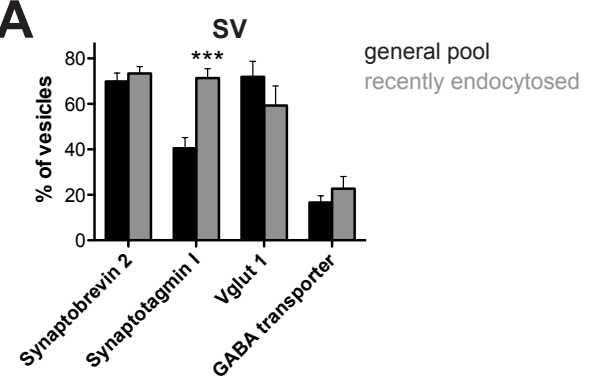

D

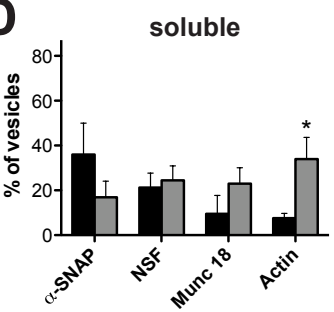

E
B

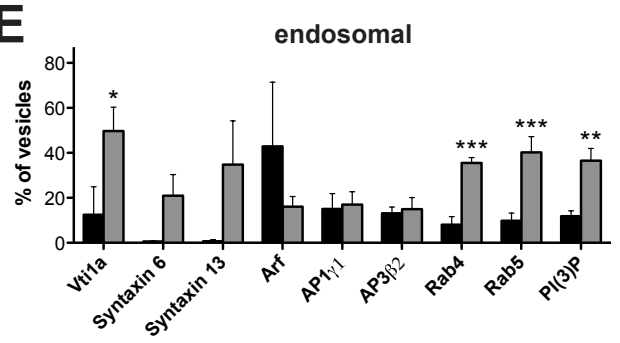

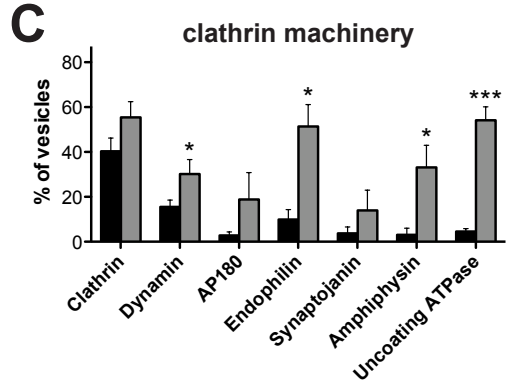

$\mathbf{F}$

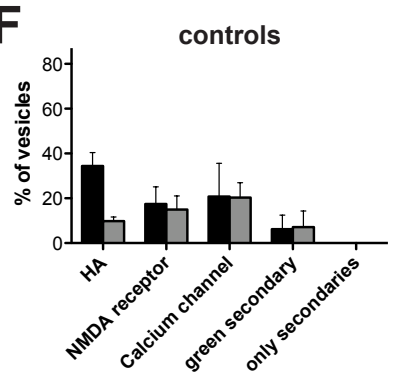

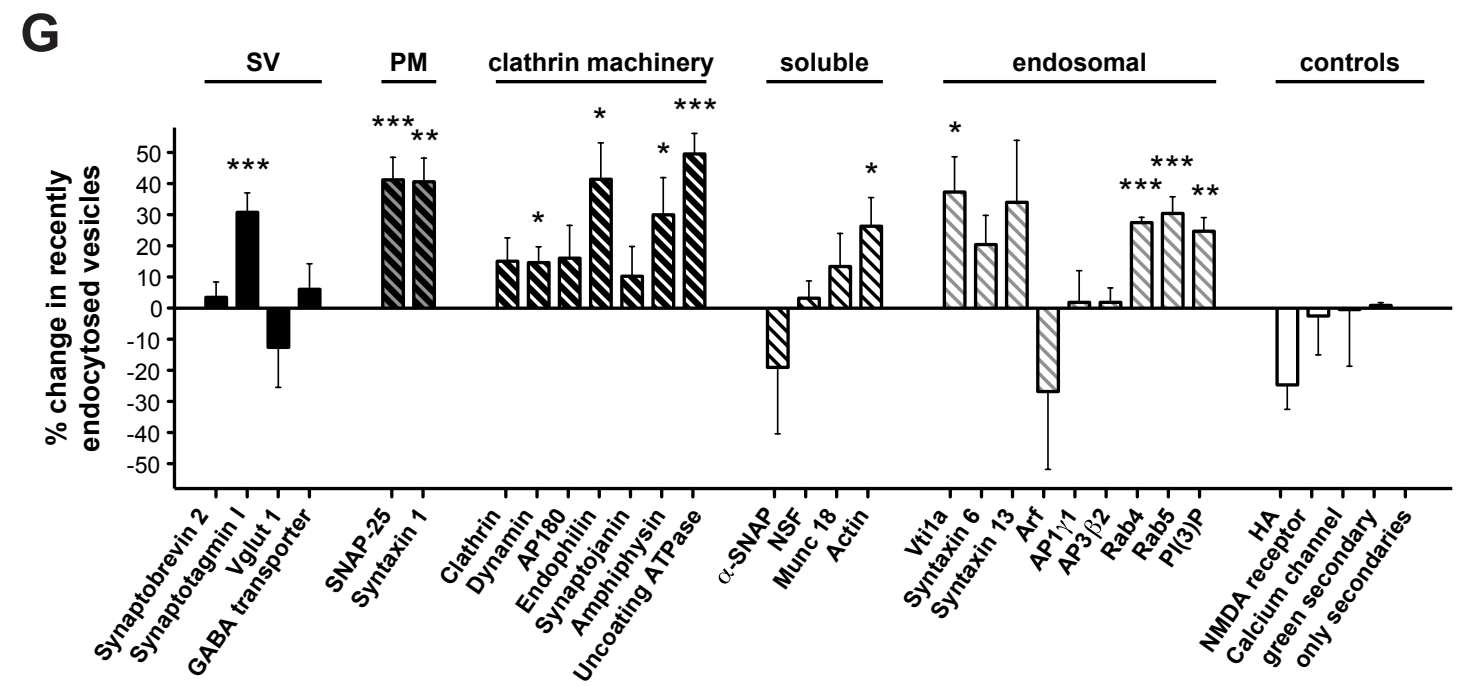

Figure 3-19: Endocytosis leads to impurities in synaptic vesicle composition

(A-F) Fractions of general pool vesicles (black) and recently endocytosed vesicles (gray) containing a variety of proteins of interest.

(A) Synaptic vesicle (SV) proteins. These proteins are typically found on similar amounts of general pool vesicles and recently endocytosed vesicles. Note that the increase observed for Synaptotagmin I is likely due to the fact that the recently endocytosed vesicles are identified by the presence of Synaptotagmin I (604.2-Oyster550, see Table 2-1), while the general pool vesicles might be lacking Synaptotagmin I and contain other Synaptotagmin isoforms instead.

(B) Plasma membrane (PM) proteins. Substantially more recently endocytosed vesicles than general pool vesicles contain the plasma membrane SNAREs SNAP-25 and Syntaxin 1.

(C) Clathrin-mediated endocytosis machinery. Some of the clathrin-mediated endocytosis machinery components are found on more recently endocytosed vesicles than general pool vesicles.

(D) Soluble proteins. These proteins are typically associated with relatively low fractions of both types of vesicles.

(E) Endosomal components. Several endosomal components are identified on more recently endocytosed vesicles than general pool vesicles. (legend continued on next page) 
(F) Controls. Stainings for a non-synaptic protein (HA) and for pre- and post-synaptic ionchannels (Calcium channel, NMDA receptor) show that the assay does not generally report an increase in the recently endocytosed vesicles. It is possible, however, that values in the range of 10-20\% are due to unspecific staining. Further controls are: 'green secondary': immunostaining for Synaptophysin as synaptic vesicle marker, combined with immunostaining with anti-mouse secondary antibodies, as protein of interest; 'only secondaries': immunostaining with anti-rabbit secondary antibodies as synaptic vesicle marker, combined with immunostaining with antimouse secondary antibodies, as protein of interest.

(G) Data from A-F is plotted as the change in protein composition for the recently endocytosed vesicles versus general pool vesicles. Positive values indicate an increase, negative values a decrease in the fraction of recently endocytosed vesicles containing the proteins of interest.

Asterisks indicate significant changes $\left({ }^{*} \mathrm{p}<0.05,{ }^{* *} \mathrm{p}<0.01,{ }^{* * *} \mathrm{p}<0.001\right.$; t-tests $)$. Data are presented as mean \pm SEM of 3-39 independent experiments (typically 3-6; Data for HA show mean \pm range of values for 2 independent experiments, data for 'only secondaries' represent one experiment).

After having verified that this procedure is capable of specifically labeling actively cycling small synaptic vesicles, we depolarized purified synaptosomes (50 mM $\mathrm{KCl}$ ) in presence of a fluorescently coupled antibody against the lumenal domain of Synaptotagmin (604.2-Oyster550; see Table 2-1; Rizzoli et al., 2006). After hypoosmotic lysis of the synaptosomes, the synaptic vesicles were adsorbed onto glass coverslips and immunostained for various markers (Figure 3-18 A). We then determined the fractions of recently endocytosed vesicles (the ones labeled with the lumenal domain anti-Synaptotagmin antibody) and general pool vesicles containing the particular markers by means of colocalization (Figure 3-18 B-C). While we found no difference between the two types of vesicles with respect to the presence of synaptic vesicle markers (Figure 3-19 A, G), clearly more of the recently endocytosed vesicles contained the plasma membrane SNAREs SNAP-25 and Syntaxin 1 (Figure 3-19 B, G). Furthermore, we found a significant enrichment of some clathrin-mediated endocytosis components (Figure 3-19 C, G) and of components of the early endosomal machinery (including the early endosomal SNARE Vti1a, the early endosomal Rabs Rab4 and Rab5, and the early endosomal lipid phosphatidyl-inositol-3-phosphate [PI(3)P]; Figure 3-19 E, G) on the recently endocytosed vesicles, confirming that a fraction of these vesicles recycle through endosomes after clathrin-mediated endocytosis. Furthermore, the fact that about $40 \%$ more of the recently endocytosed vesicles contain the plasma membrane SNAREs SNAP-25 and Syntaxin 1 (Figure 3-19 G) indicates that the probability of retrieval of a "dirty" vesicle is about 0.4, as we assumed for the Monte Carlo modeling of RRP release Figure 3-14. It is noteworthy that whenever we detected significant increases for the recently endocytosed vesicles, the increase was around 3040\% (Figure 3-19 G), indicating that the vesicles enriched in the plasma membrane SNAREs could be identical to the ones being associated with the other enriched factors. 
We conclude that it is the process of endocytosis that leads to impurities in synaptic vesicle composition, and we therefore identify endocytosis as the step in vesicle recycling necessitating endosomal sorting for the RRP vesicles.

A

PC12 Endosomes

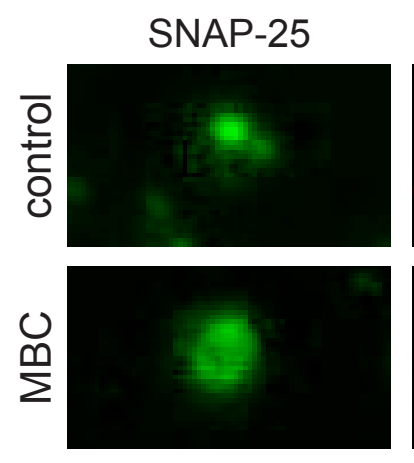

B

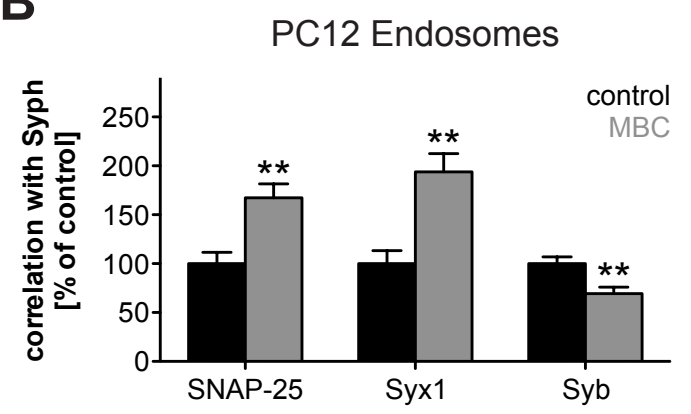

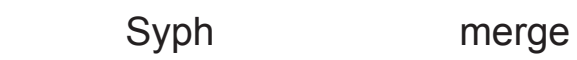
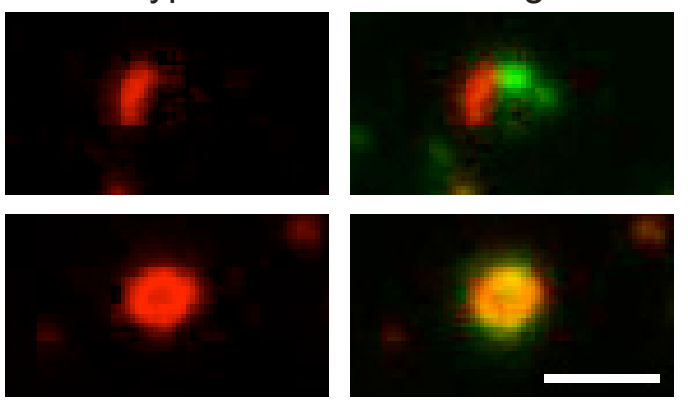

C

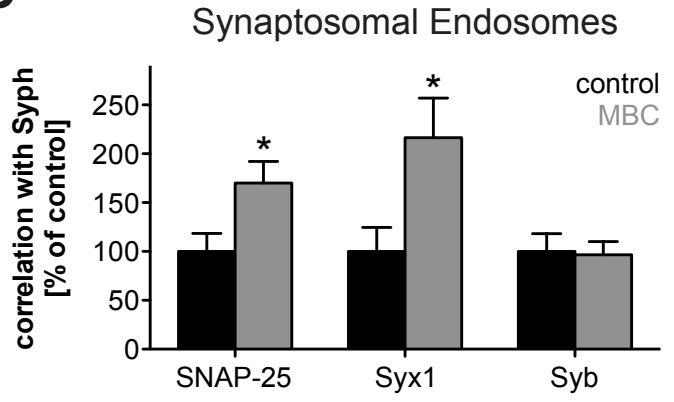

Figure 3-20: Plasma membrane and synaptic vesicle markers reside in different domains in the endosome membrane

(A) PC12 bona fide endosomes were immunostained for SNAP-25 (green; rabbit polyclonal, see Table 2-1) and Synaptophysin (red; mouse clone 7.2, see Table 2-1), and imaged by 2-color STED microscopy. Upper panels show untreated (control) endosomes; lower panels show endosomes incubated with methyl- $\beta$-cyclodextrin (MBC) for 30 minutes to extract cholesterol from the membranes (Geumann et al., 2009). Scale $=500 \mathrm{~nm}$.

(B-C) Quantification of the Synaptophysin/SNAP-25 (as in A), Synaptophysin/Syntaxin 1 (G96 polyclonal / mouse clone 78.1; see Table 2-1) and Synaptophysin/Synaptobrevin (G96 polyclonal / mouse clone 69.1; see Table 2-1) correlation in PC12 endosomes (B) and synaptosomal endosomes (C). The Pearson's correlation coefficient was calculated for 19 to 176 endosomes (typically $\sim 120$ for PC12 endosomes and $\sim 40$ for synaptosomal endosomes); values are expressed as percent of control (black). Asterisks indicate significant changes $\left({ }^{*} \mathrm{p}<0.05\right.$, $\left.{ }^{* *} \mathrm{p}<0.01\right)$. Note that cholesterol extraction by MBC increases the correlation between Synaptophysin and the plasma membrane proteins SNAP-25 and Syntaxin 1, but not between the two synaptic vesicle proteins Synaptophysin and Synaptobrevin.

Extraction experiments were performed with the help of C. Schäfer (STED Microscopy of Synaptic Function, European Neuroscience Institute Göttingen). 


\subsection{How Can Endosomes Sort Synaptic Vesicle Components?}

The results obtained so far consistently reported and solidified endosomal function/fusion to be required for the recycling of RRP vesicles. Although we found that endocytosed vesicles have a propensity to be retrieved containing plasma membrane impurities, it is unclear how the endosome would sort synaptic vesicles and rid them of these impurities. That, however, this kind of sorting is likely to happen is highlighted by the combined findings of the in vitro immunoassay presented above and the Monte Carlo model of RRP release: if "dirty" vesicles were allowed to accumulate without sorting, RRP release would be drastically reduced - a situation not encountered with intact endosomal function (see Figure 3-14 B).

To assess whether formation of lipid raft-like membrane domains contributes to the separation of different components in the endosome, we investigated isolated endosomes (either bona fide early endosomes purified from neuroendocrine PC12 cells, Rizzoli et al., 2006, or synaptosomal organelles) by 2-color STED microscopy (see section 2.3.2.3). We found the synaptic vesicle marker Synaptophysin and the plasma membrane markers Syntaxin 1 or SNAP-25 to largely avoid each other in endosomal membranes, as shown by their poor colocalization (Figure 3-20 A, upper panels). Interestingly, extraction of cholesterol from the endosomal membranes by incubation with methyl- $\beta$-cyclodextrin (MBC; Geumann et al., 2009) resulted in partial colocalization of synaptic vesicle and plasma membrane markers (Figure 3-20 A-C). To the contrary, the colocalization of the two synaptic vesicle proteins Synaptophysin and Synaptobrevin decreased slightly upon cholesterol extraction (at least in PC12 endosomes; Figure 3-20 B-C). Overall, these findings suggest a key role for cholesterol in the organization and integrity of the endosomal membrane and thus for the recycling of RRP vesicles through endosomes. 


\section{Discussion}

In this study, we have thoroughly investigated the involvement of endosomes in synaptic vesicle recycling. Our results clearly suggest endosomal sorting to be of major relevance, at least for the RRP vesicles. The implications of our results will be discussed along with the open questions in the following sections.

\subsection{Exchange of Molecules during Vesicle Recycling}

Our 2-color STED colocalization measurements of Synaptotagmin and Synaptophysin upon exocytosis revealed that these two prominent components of synaptic vesicles remain co-clustered in the plasma membrane (Figure 3-16). There are a number of reports that are in agreement with our finding: first, Synaptophysin was not found to accumulate in the plasma membrane of frog neuromuscular junctions after prolonged activity triggered by alpha-latrotoxin (which opens pre-synaptic calcium channels; Valtorta et al., 1988); second, the plasma membrane SNARE Syntaxin 1 was not observed to cycle between the plasma membrane and synaptic vesicles in hippocampal neurons, as indicated by the constant surface fluorescence of Syntaxin 1-pHluorin upon recycling pool $(20 \mathrm{~Hz} / 30$ seconds) or even much stronger $(100 \mathrm{~Hz} / 30$ seconds) stimulation (Mitchell and Ryan, 2004); third, studying single vesicle fusion and retrieval events using SynaptopHluorin detected "stranded" vesicles that remained in the plasma membrane for more than 45 seconds before being retrieved with unchanged fluorescence quantal size (Gandhi and Stevens, 2003); fourth, Synaptotagmin was found to remain in clusters corresponding to the complement of single synaptic vesicles after exocytosis when investigated by STED microscopy (Willig et al., 2006); fifth, when Synaptotagmin was labeled with two different fluorescent antibodies, the two pools did not seem to intermix during vesicle recycling (Opazo et al., 2010).

In combination with our additional finding that synaptic vesicle components persist as multi-component patches upon exocytosis, the implications from these results are that no substantial mixing of plasma membrane and vesicle components takes place in the plasma membrane. Despite these agreements, this conclusion is in discrepancy with some other previous reports, which are therefore further discussed below.

Ryan and coworkers studied the intermixing of Synaptobrevin residing in synaptic vesicles with the Synaptobrevin surface pool by using a SynaptopHluorin-based imaging approach (Fernandez-Alfonso et al., 2006). They first applied a recycling pool stimulus to hippocampal neurons expressing SynaptopHluorin to determine the 
fluorescence response generated by vesicles featuring only intact pHluorin molecules. They next bleached all surface-exposed pHluorin molecules and applied another recycling pool stimulus to deliver new non-bleached pHluorin to the surface. A subsequent extended rest period allowed for the intermixing of bleached and nonbleached SynaptopHluorin and for the endocytosis of vesicles. A third round of recycling pool stimulation resulted in a $\sim 50 \%$ reduced pHluorin response, when compared with the response to the initial pre-bleaching stimulus, which was interpreted as intermixing of bleached and non-bleached SynaptopHluorin on the surface and subsequent release of vesicles containing only $\sim 50 \%$ intact (non-bleached) pHluorin. Despite the elegant approach and the seemingly logical interpretations, there is a major pitfall to these experiments. As the pHluorin experiments rely on bulk responses to stimulation, Ryan and coworkers cannot make a crucial distinction between the following two scenarios: (1) exocytosis of half-bleached vesicles (containing the same numbers of bleached and non-bleached pHluorin molecules), indicative of intermixing in the plasma membrane; (2) exocytosis of equal numbers of fully bleached vesicles (containing only bleached pHluorin molecules) and fully non-bleached vesicles (containing only non-bleached pHluorin molecules), indicative of no intermixing in the plasma membrane. Due to this shortcoming, the conclusion from this report cannot be sustained and thus does conflict with our present finding.

In a different study, SynaptopHluorin harboring a cleavage site between the pHluorin and Synaptobrevin moieties was used to investigate the retrieval of synaptic vesicles in hippocampal cultures (Wienisch and Klingauf, 2006). Wienisch and Klingauf cleaved the surface-exposed SynaptopHluorin and then stimulated at $20 \mathrm{~Hz}$ for 20 seconds to deliver non-cleaved SynaptopHluorin to the plasma membrane. By monitoring the spatio-temporal distribution of the released SynaptopHluorin, they discovered spreading of the SynaptopHluorin in the plasma membrane, indicative of an intermixing of vesicle and plasma membrane material. Furthermore, Wienisch and Klingauf observed that after having cleaved surface-exposed SynaptopHluorin, fluorescence did not return to baseline after a $20 \mathrm{~Hz} / 5$ seconds stimulation train, which they interpreted as indicative of compensatory endocytosis of pre-cleaved SynaptopHluorin (which would not contribute to the fluorescence decrease caused by re-acidification of internalized vesicles). In agreement with their supposition that the vesicle- and surface-pool of SynaptopHluorin intermix in the plasma membrane, they observed complete return to baseline fluorescence when they allowed prolonged stimulation (5 Hz/ 40 seconds) to equilibrate surface-exposed cleaved SynaptopHluorin with non-cleaved vesicular SynaptopHluorin, before applying the $20 \mathrm{~Hz} / 5$ seconds 
stimulus. Although seemingly incompatible at first glance, these findings can be readily reconciled with our present results: Wienisch and Klingauf suggested that the incomplete return to baseline fluorescence could also be accounted for by "readily retrievable" vesicle patches (containing cleaved SynaptopHluorin), which would be preferentially retrieved during mild stimulation (up to $20 \mathrm{~Hz} / 2$ seconds). This is also in agreement with the recent finding that mild stimulation does not seem to cause diffusion of Synaptotagmin out of boutonal areas, while recycling pool stimulation caused substantial diffusion (Opazo et al., 2010). This latter report also discovered that the pHluorin-chimeras of synaptic vesicle proteins are present in rather diffuse organization in the plasma membrane, while their native counterparts appeared clustered - thus the diffusion observed in SynaptopHluorin experiments (Wienisch and Klingauf, 2006) might be an artifact of improper protein clustering, induced by the pHluorin tag. As a concluding remark it should be noted that the observed co-clustering of Synaptotagmin and Synaptophysin cannot exclude the possibility of vesicles breaking apart into several sub-vesicular patches, as the Synaptotagmin/Synaptophysin clusters exhibited diameters around the resolution limit of the used 2-color STED setup ( $\sim 60$ $\mathrm{nm}$; so that smaller clusters would go undetected). However, the fact that Synaptotagmin has already been shown to remain in patches containing the full Synaptotagmin complement of a single vesicle (Willig et al., 2006), this seems unlikely.

Our conclusion of synaptic vesicles persisting as multi-component patches has important implications for the recycling of synaptic vesicles. In principle, this clustering of vesicular components in the plasma membrane would allow the endocytosis machinery to retrieve intact synaptic vesicles, which would only need to be refilled with neurotransmitter to be ready for their next round of fusion (as suggested by Takei et al., 1996). When we compared the composition of the general pool of vesicles with that of the recently endocytosed synaptic vesicles in synaptosomal preparations (Figure 3-19), we found the recently endocytosed vesicles to be enriched in plasma membrane material (Syntaxin 1 and SNAP-25). This is remarkable in view of the aforementioned results, because it implies that the endocytosis step somehow causes intermixing of plasma membrane and vesicle components. We propose that the vesicle components remain largely co-clustered in the plasma membrane, but that the clathrin machinery only imperfectly retrieves these clusters. This is likely due to the fact that of the large number of synaptic vesicle components, only few are able to interact directly with the clathrin machinery (Jung and Haucke, 2007). This would lead to internalization of vesicles containing normal amounts of some typical synaptic vesicle components (most notably Synaptotagmin; Jung and Haucke, 2007), but could by chance also contain sub- 
normal amounts of others. Thus, a retrieved vesicle might be lacking sufficient amounts of essential fusion proteins, so that it loses its fusion competence (see below).

Interestingly, Richard Tsien and coworkers suggested a model where several rounds of kiss-and-run fusion of an individual vesicle could be followed by a full collapse fusion / clathrin-mediated endocytosis event (Zhang et al., 2009). Irrespective of whether their claims of kiss-and-run are correct, we would like to adopt their concept of primary and secondary recycling pathways: normally, the retrieved vesicles are sufficiently enriched in all essential synaptic vesicle components, so that another round of fusion can be readily accomplished after neurotransmitter refilling; if, however, the retrieved vesicle lacks some of the required components, it might fall back to a secondary recycling pathway, allowing for replenishment with the missing components. From our results, we would argue that this secondary pathway involves fusion to an endosomal compartment, from which new fusion-competent vesicles can bud (see the following section).

\subsection{Mechanisms of Endosomal Sorting of Synaptic Vesicles}

Several of our experiments confirmed the involvement of endosomes in synaptic vesicle recycling in cultured hippocampal neurons. First, we observed substantial release of the pHluorin-chimeras of the endosomal SNAREs Syntaxin 6, Syntaxin 13 and Vti1a upon electrical stimulation releasing either the RRP or the recycling pool of vesicles (Figure 3-3). Second, photo-oxidation experiments and the analogous Qdot experiments revealed the transient fusion of endocytosed vesicles to an endosome-like organelle and the subsequent budding of small vesicles from this compartment (see Figure 3-4 and Figure 3-5). Third, synaptic vesicles fuse with bona fide endosomes, as shown by the colocalization of internalized anti-Synaptotagmin antibody with Rab5-positive endosomes (see Figure 3-9 and Figure 3-10). Fourth, inhibition of endosomal function by expression of soluble Syntaxin 13 fragment drastically reduced RRP size (Figure 3-12). Fifth, recently endocytosed vesicles in synaptosomes are enriched in endosomal proteins and lipids, when compared with the general pool of vesicles (Figure 3-19).

These experiments establish several important aspects of synaptic vesicle recycling beyond the crude observation that synaptic vesicles can fuse with early endosomes (which was to be expected in view of earlier in vitro experiments; Rizzoli et al., 2006). First and foremost, the photo-oxidation experiments show that endosomal recycling is not necessarily a slow pathway, as originally proposed (Heuser and Reese, 1973). We now identified this recycling pathway as a fast pathway, requiring 30 seconds 
for the completion of a cycle, about as fast as kiss-and-run recycling (Aravanis et al., 2003). In view of the fact that endosomal recycling will most likely not be required after every round of fusion (see above), this timescale should be sufficient to maintain neurotransmitter release also during heavy bursts of activity. Furthermore, it is striking that the vesicles fused to endosomes after a RRP releasing stimulus (20 Hz / 2 seconds) in the photo-oxidation experiments correspond to about $70 \%$ of the RRP vesicles in this preparation. This is in line with the notion that not every vesicle fusion event culminates in the fusion to an endosome, likely due to the fact that vesicles are only retrieved as "dirty" vesicles in $40 \%$ of all cases (as shown by the in vitro immunoassay experiments; Figure 3-19).

Although our results confirm endosomal recycling of synaptic vesicles, the factors required for budding of vesicles from the endosome remain to be identified. Studies on microvesicle formation in PC12 cells suggest two potential factors (Lichtenstein et al., 1998), but it remains unclear whether these would be universal factors also involved in vesicles budding from neuronal endosomes.

Another open question concerns the mechanisms of sorting at the endosome. From what we know about receptor trafficking via endosomes (see section 1.2), the simplest mechanism to propose would be geometric sorting combined with raft-like membrane arrangements. In favor of this hypothesis are the co-clustering of the synaptic vesicle proteins Synaptotagmin and Synaptophysin (Figure 3-16) in the plasma membrane, and the co-clustering of the vesicle proteins Synaptobrevin and Synaptophysin in endosomes (Figure 3-20). It is likely that these clusters are not only protein-related but also involve preferences for different lipid components, reminiscent of membrane rafts (Simons and Ikonen, 1997). This possibility has been recently tested in purified endosomes from PC12 cells (Geumann et al., 2009), with the conclusion being that Synaptophysin and several SNAREs form cholesterol-dependent domains in the endosomal membrane. Interestingly, these domains were not affected by fusion of the endosomes with artificial liposomes, irrespective of the lipid composition of the liposomes. The role of domains is now reinforced by our finding that the poor colocalization of the plasma membrane proteins SNAP-25 and Syntaxin 1 with the vesicle protein Synaptophysin increased upon extraction of cholesterol from the endosomes, while the same treatment seemed to decrease the colocalization of Synaptobrevin with Synaptophysin. We propose that the formation of synaptic vesiclelike membrane domains in the endosome may assist the formation of new fusioncompetent synaptic vesicles from the endosomes. Whether vesicle components can be actively targeted to those domains will require further investigation. 


\subsection{Synaptic Vesicle Pools and Endosomal Recycling}

As mentioned above, our results imply that endosomal recycling is not equally important for all synaptic vesicles. Using the pHluorin-chimeras of endosomal SNAREs, we investigated the mobilization of these SNAREs upon electrical stimulation releasing either the RRP (20 Hz / 2 seconds) or the recycling pool of vesicles (20 Hz / 30 seconds). These experiments showed that (compared to the mobilization of Synaptobrevin, which is expected to be present on all vesicles) a relatively large amount of the endosomal SNAREs is mobilized during RRP stimulation, whereas recycling pool stimulation only caused slightly higher mobilization of these SNAREs. This was indicative of the fact that the functionally defined vesicle pools might also differ in terms of molecular composition. It seemed as if the majority of RRP vesicles contained the endosomal SNAREs, while the recycling pool vesicles do not contain substantial amounts of these proteins (as indicated by the small additional mobilization of endosomal SNAREs upon recycling pool stimulation). In support of this finding, endocytosed Synaptotagmin molecules reached bona fide endosomes in hippocampal neurons expressing Rab5-GFP variants upon RRP stimulation, and the amount of colocalization increased only marginally upon recycling pool stimulation. Taken together, we could hypothesize that the molecular difference between RRP and recycling pool vesicles is connected to the functional difference in their recycling: the RRP vesicles contain endosomal SNAREs, which together with the early endosomal fusion Rab, Rab5, (that we also found enriched on recently endocytosed vesicles; Figure 3-19) enable these vesicles to fuse to early endosomes, where their molecular composition could be maintained via yet to be determined sorting mechanisms (see above). In any case, as we observed a $60 \%$ decrease in the RRP size upon inhibition of endosomal fusion (using soluble Syntaxin 13 fragments; Figure 3-12), the importance of endosomal recycling, especially for the RRP, cannot be neglected. The results from these latter experiments allow another conclusion. Assuming a RRP size of 10 vesicles and a recycling pool size of 20 vesicles (which corresponds to the pool sizes in hippocampal neurons; Rizzoli and Betz, 2005), a $60 \%$ reduction in the RRP corresponds to 6 vesicles. Intriguingly, if these vesicles were to integrate into the recycling pool of vesicles, its size would in turn increase by $30 \%$, which is close to what we observed ( $25 \%$; Figure $3-12)$. The fact that we observed no effect on the total number of vesicles upon inhibition of endosomal fusion is in favor of this "pool conversion".

That the RRP vesicles preferentially recycle through endosomes (and that they contain endosomal SNAREs) constitutes one of the first reported mechanistic (and 
molecular) differences between the functionally defined vesicle pools. Although Synapsin has been identified as an immobilizing factor cross-linking the reserve pool vesicles, contradictory observations rendered this notion inconclusive (Rizzoli and Betz, 2005).

The fact that we also observed an increase in the spontaneous exocytosis of synaptic vesicles after inhibition of endosomal function could be attributed to the fact that the RRP is so heavily affected by the inhibition of endosomal fusion. If the endosome is normally required to maintain a tightly controlled pool of fusioncompetent vesicles, inhibition of this function will inevitably diminish the synapse's ability to respond to incoming stimuli. The Monte Caro modeling actually predicts that even as low stimulation frequencies as $0.5 \mathrm{~Hz}$ would already reduce the RRP by about $40 \%$ if endosomal sorting were non-functional (Figure 3-14). Thus, the increased rate of spontaneous fusion could simply be a way of coping with "dirty" RRP vesicles in absence of a better mechanism. By exhibiting higher spontaneous fusion rates, the synapse might try to take advantage of its imperfect endocytosis system and its inbuilt tendency to mix components - just by chance, some vesicles might pick up the required components to become competent RRP vesicles again, so that they can participate in evoked release.

These considerations could partially change our perspective on the cause-andeffect relation between endosomes and vesicle pools: we should think in terms of "RRP vesicles are RRP vesicles, because they recycle through endosomes" and not in terms of "RRP vesicles recycle through endosomes, because they are RRP vesicles". Affiliation with the classical functional vesicle pools only constitutes a classification of, rather than a property of, synaptic vesicles. Thus it follows that those vesicles, which are not tightly controlled with respect to molecular composition, will be functionally classified as recycling pool vesicles.

\subsection{Perspectives for Synaptic Vesicle Recycling}

Why is endosomal recycling especially important for RRP vesicles? We propose that these are the only vesicles that are directly required for maintenance of evoked neurotransmitter release. The RRP constitutes those vesicles that are released first upon activity. They might in fact be the only vesicles drawn from under physiological activity, as the reserve pool can only be mobilized in vitro upon heavy, prolonged stimulation. In agreement with this notion, we found similar amounts of vesicles colocalizing with bona fide endosomes both upon inherent culture activity and after a RRP releasing electrical stimulus (Figure 3-9), arguing that these cells normally (at least in culture) only use the 
RRP vesicles for neurotransmitter release. That the colocalization was slightly higher in the stimulated preparations can be explained by the defined sampling after a brief RRP or recycling pool stimulus (10 seconds post-stimulus, as identified in the photooxidation experiments). A similar consideration holds for the slight increase in correlation upon stimulation in the live-STED experiments (Figure 3-10): although inherent culture activity draws vesicles from the RRP, externally applied recycling pool stimulation quickly depletes the RRP (and the recycling pool vesicles), leading to more synchronized transit of vesicles to the endosome.

The high level of activity required to release the recycling pool (and even more so the reserve pool) in vitro may never be reached in vivo, thus calling for an alternative functional relevance of the recycling/reserve pool vesicles. If they are normally not used for neurotransmitter release, what is the purpose of their presence in the synapse? We propose that they may serve as a "reservoir" of molecules, rather than organelles intended for release (personal communication: A. Denker, STED Microscopy of Synaptic Function, European Neuroscience Institute Göttingen). By resembling/mimicking functional RRP vesicles, the "reservoir vesicles" can interact with many of the soluble factors involved in synaptic vesicle recycling (including Rabs and their effectors, clathrin and their adaptor proteins, NSF and $\alpha$-SNAP, etc.) and keep them from diffusing out of the pre-synaptic space. This way, the majority of all vesicles in a synapse might be of extraordinary relevance for synaptic vesicle exocytosis, but in a much more indirect way than previously believed. Whether this hypothesis can be corroborated, and whether this hypothesis maintains a distinction between "reservoir vesicles" and a reserve pool remains to be seen.

The results from this study, to some extent, make a kiss-and-run recycling pathway superfluous. As mentioned in the introduction, no molecular determinants of kiss-and-run have been identified. It remains a hypothetical model that is highly contested. The theoretical advantages this model has to offer are mainly the speed of recycling and the inbuilt maintenance of vesicle identity. However, our results show that even with endosomal recycling the speed of recycling after full-collapse fusion is on the same timescale as proposed for kiss-and-run. As our data implies that endosomal sorting does not necessarily take place after every round of fusion, the average recycling time of classical full-collapse fusion will not drastically exceed the time required for kiss-and-run recycling. Together with the concept of readily retrievable vesicles (Fernandez-Alfonso et al., 2006; Wienisch and Klingauf, 2006), these considerations negate the possible advantage in speed typically attributed to kiss-and-run recycling. The second potential advantage of kiss-and-run, maintenance of vesicular identity, is 
also open for discussion in view of the present findings. We found vesicle components to remain largely co-clustered both in the plasma membrane and in endosomes. Taken the well-established clathrin-mediated endocytosis pathway (which can at least recognize Synaptotagmin), the potential formation of membrane raft arrangements enriched in synaptic vesicle material, and the established role of endosomes in sorting, the endosomal recycling pathway seems at least as well-suited for maintaining vesicle identity as a potential kiss-and-run mechanism. As a matter of fact, a recent report from the group of Richard Tsien suggests that kiss-and-run events can be followed by fullcollapse fusion of the same vesicle (Zhang et al., 2009). Maybe it is not kiss-and-run that maintains the molecular identity, but endosomal recycling that is required for reestablishing the vesicle composition (also) after kiss-and-run events - the sorting mechanism that has to be proposed to account for molecular maintenance during kissand-run has to prevent lateral diffusion of vesicle components into the plasma membrane and could involve a trans-membrane clamp protein flanking the fusion pore (although this is purely speculative in view of the fact that FM dye was shown to readily diffuse away from fused vesicles; Zenisek et al., 2002). 


\section{SUMMARY AND CONCLUSIONS}

The hallmark of neurotransmission at the chemical synapse is the release of low molecular weight neurotransmitters from synaptic vesicles. Synaptic vesicles are small $(\sim 42 \mathrm{~nm})$ membrane-bounded, neurotransmitter-filled organelles residing in the presynaptic nerve terminal (bouton). Upon arrival of an action potential at the pre-synaptic bouton, calcium influx through voltage-gated calcium channels triggers the fusion (exocytosis) of synaptic vesicles with the plasma membrane at a specialized area termed active zone. The exocytosis of synaptic vesicles causes release of the neurotransmitter into the synaptic cleft, the tight space between the pre- and post-synaptic cells. Here, the transmitter spreads by diffusion and binds to surface receptors on the post-synaptic cell, eventually eliciting an electrical response. The fused synaptic vesicles are retrieved into the pre-synaptic nerve terminal (endocytosis) and are refilled with neurotransmitter before being integrated into the synaptic vesicle pool.

The pre-synaptic pool of synaptic vesicles has been classically described as consisting of three functionally defined pools: (1) The readily releasable pool (RRP) vesicles are the most fusion-competent vesicles and they are the first ones to undergo fusion upon stimulation, in line with the description that they correspond to the vesicles morphologically docked at the active zone; in cultured hippocampal neurons, this pool only constitutes $\sim 5 \%$ of all vesicles ( 10 out of 200 ) and it can be released by a brief 20 $\mathrm{Hz} / 2$ seconds electrical stimulus. (2) The recycling pool measures $\sim 10 \%$ of all vesicles (20 out of 200) in hippocampal cultures and it can be released only by prolonged $20 \mathrm{~Hz}$ / 30 seconds stimulation in vitro; whether this pool of vesicles exhibits any molecular differences from the RRP remains to be determined. (3) The reserve pool of vesicles ( $\sim 85 \% ; 170$ out of 200 ) cannot be stimulated to release in cultured hippocampal neurons and its mobilization requires strong, non-physiological stimulation in other preparations.

Various models of synaptic vesicle recycling including "kiss-and-run", clathrinmediated endocytosis, endosomal recycling and bulk endocytosis have been proposed in the past. In the most recent model, kiss-and-run, vesicles are thought to fuse transiently to the plasma membrane without loss of identity via the opening of a fusion pore and the subsequent retraction of the vesicles into the bouton. The classical mode of exocytosis, on the other hand, involves complete collapse of the vesicles into the plasma membrane upon fusion of the two lipid bilayers. Retrieval of the vesicles is then achieved via 
clathrin-coated pits and vesicles, and the vesicles can integrate into the synaptic vesicle cluster by simply uncoating or by recycling through an endosomal compartment. Another recycling pathway, bulk endocytosis, also involves full-collapse fusion of vesicles, but is thought to retrieve multiple vesicles at once by forming large plasma membrane invaginations, which can pinch-off and form internal cisternae, from which new vesicle can form by budding. Bulk endocytosis has been described to be mainly functional under circumstances of heavy exocytotic load. Whether the identity of fused synaptic vesicles in the plasma membrane is maintained or whether the components intermix with plasma membrane components is still a matter of debate.

In this study we set out to investigate the involvement of endosomes in the recycling of synaptic vesicles by employing several advanced imaging approaches. The main questions to be addressed were: (1) Do endosomal proteins cycle between internal organelles and the plasma membrane upon stimulation? (2) Do synaptic vesicles fuse with endosomes during recycling? (3) Does inhibition of endosomal function have an effect on any of the synaptic vesicle pools? (4) Do vesicle and plasma membrane components intermix during synaptic vesicle recycling? (5) How could endosomal sorting maintain synaptic vesicle identity?

\subsection{Do Endosomal Proteins Cycle Between Internal Organelles and the Plasma Membrane upon Stimulation?}

To investigate the cycling of endosomal proteins between internal organelles and the plasma membrane, we developed three novel reporters of vesicle exocytosis. These reporters were generated by fusing a pH-sensitive variant of GFP (pHluorin) to the lumenal domain of the early endosomal SNARE proteins Vti1a, Syntaxin 13 and Syntaxin 6. As the pHluorin-moiety is quenched at the acidic $\mathrm{pH}$ of internal organelles (synaptic vesicles, endosomes), but brightly fluorescent at the neutral $\mathrm{pH}$ of the extracellular medium, these new pHluorin-chimeras can serve as reporters of exocytosis of vesicles containing these endosomal SNAREs.

We expressed our reporters in hippocampal neurons and verified that they are properly expressed on internal organelles, with the surface fraction of all three pHluorin-chimeras being less than $10 \%$. When we stimulated the neurons to release the RRP, we could detect a substantial release of all three endosomal pHluorins, comparable to the release of the pHluorin-variant of the synaptic vesicle protein Synaptobrevin (SynaptopHluorin). When we applied a recycling pool releasing stimulus, we only 
observed slightly higher release of the endosomal SNAREs, while the increase in SynaptopHluorin release was sizeable. These results not only established that endosomal SNAREs cycle together with recycling synaptic vesicles, but it also suggested that endosomal SNAREs are predominantly present on the RRP vesicles.

\subsection{Do Synaptic Vesicles Fuse with Endosomes During Recycling?}

After the pHluorin experiments suggested an involvement of endosomal SNAREs in the recycling of RRP vesicles, we went on to investigate the passage of recycling synaptic vesicles through endosomes by a number of assays.

We first labeled RRP vesicles in hippocampal neurons with the styryl dye FM 143 and allowed a resting period of 4,10 or 30 seconds, before processing the preparations for photo-oxidation and electron microscopy. The photo-oxidation generates an electron-dense precipitate (in the places where FM dye labeling is present) that can be detected in electron microscopy. While all labeled organelles where small after 4 seconds, larger labeled structures appeared after 10 seconds and disappeared again after 30 seconds. The simplest interpretation of these results is that endocytosed RRP vesicles fuse to endosomes, which later bud small vesicles.

As the photo-oxidation results could not clearly identify the observed large labeled structures as endosomes due to the lack of endosomal markers, we turned to expression of the early endosomal protein Rab5 (fused to GFP). By following several different labeling schemes with fluorescent antibodies against the synaptic vesicle protein Synaptotagmin, we could corroborate that synaptic vesicles fuse with bona fide Rab5-positive endosomes. Interestingly, these experiments consistently showed no (or very little) increase in the amount of Synaptotagmin reaching the endosomes, when comparing recycling pool stimulation with RRP stimulation.

\subsection{Does Inhibition of Endosomal Function Have an Effect on Any of the Synaptic Vesicle Pools?}

As several of the results suggested a role of endosomal fusion for the RRP vesicles, but not for the recycling pool vesicles, we performed a different set of experiments focusing on vesicle pool related effects of inhibiting endosomal function. To this end, we expressed in hippocampal neurons soluble fragments of the early endosomal SNARE Syntaxin 13 , which engages its cognate SNARE partners on the endosomal and synaptic 
vesicle membranes in non-fusogenic SNARE complexes, therefore specifically inhibiting the fusion of synaptic vesicles with endosomes. While the total amount of synaptic vesicles was not affected, we observed a drastic 60\% decrease in the RRP size, concurrent with a $25 \%$ increase in recycling pool size and a $50 \%$ increase in spontaneous exocytosis. By simulating RRP release in absence of endosomal recycling in a Monte Carlo model, we determined the release probability of the non-sorted retrieved vesicles to be about 6-fold lower than the release probability or normal RRP vesicles.

We conclude that endosomal function is of crucial importance for the maintenance of the RRP. The observed increase in recycling pool is likely due to a conversion of RRP vesicles to recycling pool vesicles, while the increase in spontaneous exocytosis could be a rescue mechanism to achieve chance-based sorting during endocytosis.

\subsection{Do Vesicle and Plasma Membrane Components Intermix during Synaptic Vesicle Recycling?}

After having established and corroborated endosomal recycling of synaptic vesicles by multiple assays, the question of which recycling step necessitates endosomal sorting remained. To address this issue, we first investigated Synaptotagmin/Synaptophysin colocalization in the plasma membrane of hippocampal neurons upon exocytosis by 2color STED microscopy. The two proteins appeared to persist as multi-component protein clusters in the plasma membrane, with no trace of substantial diffusion. We next developed an in vitro immunoassay to compare the composition of isolated general pool vesicles and recently endocytosed vesicles purified from rat brain synaptosomes. We identified endocytosis as the recycling step likely inducing the requirement for endosomal sorting, as the recently endocytosed vesicles were enriched in several contaminating components, including the plasma membrane SNAREs SNAP-25 and Syntaxin 1, the early endosomal proteins Vti1a, Rab4, Rab5 and the early endosomal lipid PI(3)P. We could tentatively conclude that about $40 \%$ of the endocytosed vesicles were retrieved as "dirty" vesicles.

\subsection{How Could Endosomal Sorting Maintain Synaptic Vesicle Identity?}

Although we could identify endocytosis to be the recycling step necessitating endosomal sorting of RRP vesicles, just how the endosome would sort the vesicular components 
remained enigmatic. As we had found Synaptotagmin and Synaptophysin to remain coclustered upon exocytosis, and as the formation of raft-like proteo-lipid domains in endosomes has been described at least for Synaptophysin (Geumann et al., 2009), we turned to endosomes purified from neuroendocrine PC12 cells or synaptosomes and analyzed the colocalization of Synaptophysin with the plasma membrane SNAREs SNAP25 and Syntaxin 1 or with the synaptic vesicle SNARE Synaptobrevin. As expected, colocalization was only appreciable for Synaptophysin/Synaptobrevin in control conditions. However, when we extracted cholesterol from the endosomal membranes by incubating with methyl- $\beta$-cyclodextrin, the colocalization of Synaptophysin with SNAP25 and Syntaxin 1 increased markedly. This was indicative of the fact that vesicle components are not only preserved as multi-component clusters in the plasma membrane, but that they also gathered as multi-component patches in the endosomal membrane. The cholesterol-dependence of the clustering furthermore highlights that different protein clusters might have preferences for different lipid environments, facilitating the sorting/separation of material in the endosome without the need for sorting/targeting sequences for each protein. We conclude that endosomal sorting is likely to be based on a geometry-based raft-assisted mechanism, but the processes governing the sorting of vesicle material in endosomes will require further in-depth investigation.

\subsection{Open Questions}

In summary, this study could successfully provide the long-awaited direct evidence for endosomal recycling of synaptic vesicles by combining novel endosomal pHluorins, photo-oxidation electron microscopy and expression of Rab5-GFP variants as markers for early endosomes. Taken together, these experiments leave no doubt that endosomes are involved in the recycling of synaptic vesicles. Exceeding our expectations, we were also able to identify a specific role of endosomal sorting for the RRP vesicles, which triggers a number of open questions (discussed below).

First, it is unclear how many copies of the endosomal SNAREs would be required per vesicle in order to establish RRP vesicle properties. Although the pHluorin experiments showed that the fractional release of endosomal SNAREs relative to the fractional release of SynaptopHluorin was higher upon RRP stimulation than upon recycling pool stimulation, the release of endosomal SNAREs continued throughout recycling pool stimulation (Figure 3-3), indicating that also recycling pool vesicles contain the endosomal SNAREs. Förster resonance energy transfer measurements of 
fusion assays with liposomes and synaptic vesicles recently showed that a single SNARE complex could be sufficient to drive in vitro membrane fusion (van den Bogaart et al., 2010). If this was also the case for fusion reactions in a cellular context, this result would argue that the number of endosomal SNAREs is similar for recycling pool and RRP vesicles, but more of the RRP vesicles contain these SNAREs. If the majority of recycling pool vesicles contained (even trace amounts) the endosomal SNAREs, we should have observed an increase in colocalization between Rab5-GFP endosomes and synaptic vesicle marker upon recycling pool stimulation (Figure 3-9; Figure 3-10). Likewise, the blocking of endosomal function by expression of soluble Syntaxin 13 fragment should have resulted in a decrease in the recycling pool size if a majority of them relied on endosomal function Figure 3-12. Although these considerations are by no means sufficient to clarify this issue, we favor a scenario of endosomal SNAREs being present mostly on RRP vesicles and only on a minority of recycling pool vesicles. We are of course aware of the fact that the endosomal SNAREs are unlikely to be the only molecular determinants of RRP vesicles. As indicated in the in vitro immunoassay, the recently endocytosed vesicles are also enriched in Rab4, Rab5 and PI(3)P (and most likely others; see Figure 3-19).

Second, the biggest piece of the puzzle is the sorting of vesicle components in the endosome. Although we could identify cholesterol-dependant clustering of synaptic vesicle material in the endosome (Figure 3-20), we don not know, for example, (1) which components are contained in these clusters, (2) if there are different types of vesicular patches, and (3) which of the components in the vesicular patches are targeted for packaging into synaptic vesicles. As even the universal clathrin-machinery seems to be able to only interact with a limited number of targets (see Jung and Haucke, 2007), it is unlikely that in-depth analysis will discover many components being targeted for vesicle budding from the endosomes. In the end it might be sufficient to rely on coincidence detection of a few components, each of which bring along a specific patch of vesicular components required for synaptic vesicle function.

Third, an aspect easily disregarded is the fate of the contaminants reaching the endosome along with the retrieved "dirty" vesicles. If they are packaged into separate non-synaptic secretory vesicles to be returned to the plasma membrane, how is the budding of these vesicles controlled versus the budding of synaptic vesicles? Otherwise, if they are packaged together with synaptic vesicle patches, how many contaminant components are tolerated and how is the amount of contaminants controlled? 


\section{BIBLIOGRAPHY}

Abbe E (1873) Beiträge zur Theorie des Mikroskops und der mikroskopischen Wahrnehmung. Archive f Mikroskop Anat 9:413-420.

Aguado F, Majo G, Ruiz-Montasell B, Canals JM, Casanova A, Marsal J, Blasi J (1996) Expression of synaptosomal-associated protein SNAP-25 in endocrine anterior pituitary cells. Eur J Cell Biol 69:351-359.

Akbergenova Y, Bykhovskaia M (2009) Stimulation-induced formation of the reserve pool of vesicles in Drosophila motor boutons. J Neurophysiol 101:2423-2433.

Alberts B (2002) Molecular biology of the cell, 4th Edition. New York: Garland Science.

Antonin W, Riedel D, von Mollard GF (2000) The SNARE Vti1a-beta is localized to small synaptic vesicles and participates in a novel SNARE complex. J Neurosci 20:5724-5732.

Aravanis AM, Pyle JL, Tsien RW (2003) Single synaptic vesicles fusing transiently and successively without loss of identity. Nature 423:643-647.

Atwood HL, Govind CK, Wu CF (1993) Differential ultrastructure of synaptic terminals on ventral longitudinal abdominal muscles in Drosophila larvae. J Neurobiol 24:1008-1024.

Barnstable CJ, Hofstein R, Akagawa K (1985) A marker of early amacrine cell development in rat retina. Brain Res 352:286-290.

Bennett MK, Calakos N, Scheller RH (1992) Syntaxin: a synaptic protein implicated in docking of synaptic vesicles at presynaptic active zones. Science 257:255-259.

Bethani I, Lang T, Geumann U, Sieber JJ, Jahn R, Rizzoli SO (2007) The specificity of SNARE pairing in biological membranes is mediated by both proof-reading and spatial segregation. EMBO J 26:3981-3992.

Bethani I, Werner A, Kadian C, Geumann U, Jahn R, Rizzoli SO (2009) Endosomal fusion upon SNARE knockdown is maintained by residual SNARE activity and enhanced docking. Traffic 10:1543-1559.

Betz WJ, Bewick GS (1992) Optical analysis of synaptic vesicle recycling at the frog neuromuscular junction. Science 255:200-203.

Betzig E, Patterson GH, Sougrat R, Lindwasser OW, Olenych S, Bonifacino JS, Davidson MW, Lippincott-Schwartz J, Hess HF (2006) Imaging intracellular fluorescent proteins at nanometer resolution. Science 313:1642-1645.

Bhalla A, Chicka MC, Chapman ER (2008) Analysis of the synaptotagmin family during reconstituted membrane fusion. Uncovering a class of inhibitory isoforms. J Biol Chem 283:21799-21807. 
Bradford MM (1976) A rapid and sensitive method for the quantitation of microgram quantities of protein utilizing the principle of protein-dye binding. Anal Biochem 72:248-254.

Brown DA, Rose JK (1992) Sorting of GPI-anchored proteins to glycolipid-enriched membrane subdomains during transport to the apical cell surface. Cell 68:533544.

Burkhardt P, Hattendorf DA, Weis WI, Fasshauer D (2008) Munc18a controls SNARE assembly through its interaction with the syntaxin N-peptide. EMBO J 27:923933.

Ceccarelli B, Hurlbut WP, Mauro A (1973) Turnover of transmitter and synaptic vesicles at the frog neuromuscular junction. J Cell Biol 57:499-524.

Chamberlain LH, Gould GW (2002) The vesicle- and target-SNARE proteins that mediate Glut4 vesicle fusion are localized in detergent-insoluble lipid rafts present on distinct intracellular membranes. J Biol Chem 277:49750-49754.

Chavrier P, Parton RG, Hauri HP, Simons K, Zerial M (1990) Localization of low molecular weight GTP binding proteins to exocytic and endocytic compartments. Cell 62:317-329.

Cheung G, Jupp 0J, Cousin MA (2010) Activity-dependent bulk endocytosis and clathrindependent endocytosis replenish specific synaptic vesicle pools in central nerve terminals. J Neurosci 30:8151-8161.

Clague MJ (1998) Molecular aspects of the endocytic pathway. Biochem J 336 ( Pt 2):271-282.

Clayton EL, Cousin MA (2008) Differential labelling of bulk endocytosis in nerve terminals by FM dyes. Neurochem Int 53:51-55.

Clayton EL, Evans GJ, Cousin MA (2008) Bulk synaptic vesicle endocytosis is rapidly triggered during strong stimulation. J Neurosci 28:6627-6632.

Clayton EL, Sue N, Smillie KJ, O'Leary T, Bache N, Cheung G, Cole AR, Wyllie DJ, Sutherland C, Robinson PJ, Cousin MA (2010) Dynamin I phosphorylation by GSK3 controls activity-dependent bulk endocytosis of synaptic vesicles. Nat Neurosci 13:845-851.

Cullen PJ (2008) Endosomal sorting and signalling: an emerging role for sorting nexins. Nat Rev Mol Cell Biol 9:574-582.

David C, McPherson PS, Mundigl O, de Camilli P (1996) A role of amphiphysin in synaptic vesicle endocytosis suggested by its binding to dynamin in nerve terminals. Proc Natl Acad Sci U S A 93:331-335.

Davletov BA, Sudhof TC (1993) A single C2 domain from synaptotagmin I is sufficient for high affinity Ca2+/phospholipid binding. J Biol Chem 268:26386-26390.

de Hoop MJ, Huber LA, Stenmark H, Williamson E, Zerial M, Parton RG, Dotti CG (1994) The involvement of the small GTP-binding protein Rab5a in neuronal endocytosis. Neuron 13:11-22. 
Delgado R, Maureira C, Oliva C, Kidokoro Y, Labarca P (2000) Size of vesicle pools, rates of mobilization, and recycling at neuromuscular synapses of a Drosophila mutant, shibire. Neuron 28:941-953.

Denker A, Krohnert K, Rizzoli SO (2009) Revisiting synaptic vesicle pool localization in the Drosophila neuromuscular junction. J Physiol.

Diril MK, Wienisch M, Jung N, Klingauf J, Haucke V (2006) Stonin 2 is an AP-2-dependent endocytic sorting adaptor for synaptotagmin internalization and recycling. Dev Cell 10:233-244.

Edeling MA, Smith C, Owen D (2006) Life of a clathrin coat: insights from clathrin and AP structures. Nat Rev Mol Cell Biol 7:32-44.

Evarts EV (1964) Temporal Patterns of Discharge of Pyramidal Tract Neurons during Sleep and Waking in the Monkey. J Neurophysiol 27:152-171.

Fasshauer D, Sutton RB, Brunger AT, Jahn R (1998) Conserved structural features of the synaptic fusion complex: SNARE proteins reclassified as Q- and R-SNAREs. Proc Natl Acad Sci U S A 95:15781-15786.

Fernandez-Alfonso T, Ryan TA (2004) The kinetics of synaptic vesicle pool depletion at CNS synaptic terminals. Neuron 41:943-953.

Fernandez-Alfonso T, Ryan TA (2006) The efficiency of the synaptic vesicle cycle at central nervous system synapses. Trends Cell Biol 16:413-420.

Fernandez-Alfonso T, Kwan R, Ryan TA (2006) Synaptic vesicles interchange their membrane proteins with a large surface reservoir during recycling. Neuron 51:179-186.

Fesce R, Grohovaz F, Valtorta F, Meldolesi J (1994) Neurotransmitter release: fusion or 'kiss-and-run'? Trends Cell Biol 4:1-4.

Fischer von Mollard G, Stahl B, Li C, Sudhof TC, Jahn R (1994) Rab proteins in regulated exocytosis. Trends Biochem Sci 19:164-168.

Foster MS, Sherrington CSS (1897) A text book of physiology, 7th , largely rev. Edition. London: Macmillan.

Frost A, Unger VM, De Camilli P (2009) The BAR domain superfamily: membranemolding macromolecules. Cell 137:191-196.

Gandhi SP, Stevens CF (2003) Three modes of synaptic vesicular recycling revealed by single-vesicle imaging. Nature 423:607-613.

Gennaro JF, Jr., Nastuk WL, Rutherford DT (1978) Reversible depletion of synaptic vesicles induced by application of high external potassium to the frog neuromuscular junction. J Physiol 280:237-247.

Geumann U, Barysch SV, Hoopmann P, Jahn R, Rizzoli SO (2008) SNARE function is not involved in early endosome docking. Mol Biol Cell 19:5327-5337.

Geumann U, Schafer C, Riedel D, Jahn R, Rizzoli SO (2009) Synaptic membrane proteins form stable microdomains in early endosomes. Microsc Res Tech. 
Glyvuk N, Tsytsyura Y, Geumann C, D'Hooge R, Huve J, Kratzke M, Baltes J, Boning D, Klingauf J, Schu P (2010) AP-1/sigma1B-adaptin mediates endosomal synaptic vesicle recycling, learning and memory. EMBO J 29:1318-1330.

Grabenbauer M, Geerts WJ, Fernadez-Rodriguez J, Hoenger A, Koster AJ, Nilsson T (2005) Correlative microscopy and electron tomography of GFP through photooxidation. Nat Methods 2:857-862.

Granseth B, Odermatt B, Royle SJ, Lagnado L (2006) Clathrin-mediated endocytosis is the dominant mechanism of vesicle retrieval at hippocampal synapses. Neuron 51:773-786.

Granseth B, Odermatt B, Royle SJ, Lagnado L (2009) Comment on "The dynamic control of kiss-and-run and vesicular reuse probed with single nanoparticles". Science 325:1499; author reply 1499.

Grant BD, Donaldson JG (2009) Pathways and mechanisms of endocytic recycling. Nat Rev Mol Cell Biol 10:597-608.

Green DJ, Gillette R (1982) Circadian rhythm of firing rate recorded from single cells in the rat suprachiasmatic brain slice. Brain Res 245:198-200.

Grigliatti TA, Hall L, Rosenbluth R, Suzuki DT (1973) Temperature-sensitive mutations in Drosophila melanogaster. XIV. A selection of immobile adults. Mol Gen Genet 120:107-114.

Gurkan C, Lapp H, Alory C, Su AI, Hogenesch JB, Balch WE (2005) Large-scale profiling of Rab GTPase trafficking networks: the membrome. Mol Biol Cell 16:3847-3864.

Haffner C, Di Paolo G, Rosenthal JA, de Camilli P (2000) Direct interaction of the $170 \mathrm{kDa}$ isoform of synaptojanin 1 with clathrin and with the clathrin adaptor AP-2. Curr Biol 10:471-474.

Hao W, Luo Z, Zheng L, Prasad K, Lafer EM (1999) AP180 and AP-2 interact directly in a complex that cooperatively assembles clathrin. J Biol Chem 274:22785-22794.

Harata N, Ryan TA, Smith SJ, Buchanan J, Tsien RW (2001a) Visualizing recycling synaptic vesicles in hippocampal neurons by FM 1-43 photoconversion. Proc Natl Acad Sci U S A 98:12748-12753.

Harata N, Pyle JL, Aravanis AM, Mozhayeva M, Kavalali ET, Tsien RW (2001b) Limited numbers of recycling vesicles in small CNS nerve terminals: implications for neural signaling and vesicular cycling. Trends Neurosci 24:637-643.

Harata NC, Choi S, Pyle JL, Aravanis AM, Tsien RW (2006) Frequency-dependent kinetics and prevalence of kiss-and-run and reuse at hippocampal synapses studied with novel quenching methods. Neuron 49:243-256.

He L, Wu XS, Mohan R, Wu LG (2006) Two modes of fusion pore opening revealed by cell-attached recordings at a synapse. Nature 444:102-105.

Heuser JE, Reese TS (1973) Evidence for recycling of synaptic vesicle membrane during transmitter release at the frog neuromuscular junction. J Cell Biol 57:315-344. 
Hoopmann P (2008) Fluorescence Microscopy Investigation of Isolated Synaptic Vesicles. In: STED Microscopy of Synaptic Function. Göttingen: Georg-AugustUniversität Göttingen.

Huttner WB, Schiebler W, Greengard P, De Camilli P (1983) Synapsin I (protein I), a nerve terminal-specific phosphoprotein. III. Its association with synaptic vesicles studied in a highly purified synaptic vesicle preparation. J Cell Biol 96:13741388.

Jahn R, Scheller RH (2006) SNAREs--engines for membrane fusion. Nat Rev Mol Cell Biol 7:631-643.

Jahn R, Schiebler W, Ouimet C, Greengard P (1985) A 38,000-dalton membrane protein (p38) present in synaptic vesicles. Proc Natl Acad Sci U S A 82:4137-4141.

Jung N, Haucke V (2007) Clathrin-mediated endocytosis at synapses. Traffic 8:11291136.

Kandel ER, Schwartz JH, Jessell TM (2000) Principles of neural science, 4th Edition. New York: McGraw-Hill, Health Professions Division.

Katzmann DJ, Odorizzi G, Emr SD (2002) Receptor downregulation and multivesicularbody sorting. Nat Rev Mol Cell Biol 3:893-905.

Klingauf J, Kavalali ET, Tsien RW (1998) Kinetics and regulation of fast endocytosis at hippocampal synapses. Nature 394:581-585.

Koenig JH, Ikeda K (1989) Disappearance and reformation of synaptic vesicle membrane upon transmitter release observed under reversible blockage of membrane retrieval. J Neurosci 9:3844-3860.

Kuromi H, Kidokoro Y (2003) Two synaptic vesicle pools, vesicle recruitment and replenishment of pools at the Drosophila neuromuscular junction. J Neurocytol 32:551-565.

Kuromi H, Honda A, Kidokoro Y (2004) Ca2+ influx through distinct routes controls exocytosis and endocytosis at drosophila presynaptic terminals. Neuron 41:101111.

Lang T, Bruns D, Wenzel D, Riedel D, Holroyd P, Thiele C, Jahn R (2001) SNAREs are concentrated in cholesterol-dependent clusters that define docking and fusion sites for exocytosis. EMBO J 20:2202-2213.

Lee SJ, Liyanage U, Bickel PE, Xia W, Lansbury PT, Jr., Kosik KS (1998) A detergentinsoluble membrane compartment contains A beta in vivo. Nat Med 4:730-734.

Lencer WI, Tsai B (2003) The intracellular voyage of cholera toxin: going retro. Trends Biochem Sci 28:639-645.

Lichte B, Veh RW, Meyer HE, Kilimann MW (1992) Amphiphysin, a novel protein associated with synaptic vesicles. EMBO J 11:2521-2530.

Lichtenstein Y, Desnos C, Faundez V, Kelly RB, Clift-O'Grady L (1998) Vesiculation and sorting from PC12-derived endosomes in vitro. Proc Natl Acad Sci U S A 95:11223-11228. 
Lodish HF (2003) Molecular cell biology, 5th Edition. New York: W.H. Freeman and Company.

Matthew WD, Tsavaler L, Reichardt LF (1981) Identification of a synaptic vesiclespecific membrane protein with a wide distribution in neuronal and neurosecretory tissue. J Cell Biol 91:257-269.

Maxfield FR, McGraw TE (2004) Endocytic recycling. Nat Rev Mol Cell Biol 5:121-132.

McBride HM, Rybin V, Murphy C, Giner A, Teasdale R, Zerial M (1999) Oligomeric complexes link Rab5 effectors with NSF and drive membrane fusion via interactions between EEA1 and syntaxin 13. Cell 98:377-386.

McPherson PS, Czernik AJ, Chilcote TJ, Onofri F, Benfenati F, Greengard P, Schlessinger J, De Camilli P (1994) Interaction of Grb2 via its Src homology 3 domains with synaptic proteins including synapsin I. Proc Natl Acad Sci U S A 91:6486-6490.

Micheva KD, Ramjaun AR, Kay BK, McPherson PS (1997) SH3 domain-dependent interactions of endophilin with amphiphysin. FEBS Lett 414:308-312.

Miesenbock G, De Angelis DA, Rothman JE (1998) Visualizing secretion and synaptic transmission with pH-sensitive green fluorescent proteins. Nature 394:192-195.

Miller TM, Heuser JE (1984) Endocytosis of synaptic vesicle membrane at the frog neuromuscular junction. J Cell Biol 98:685-698.

Mitchell SJ, Ryan TA (2004) Syntaxin-1A is excluded from recycling synaptic vesicles at nerve terminals. J Neurosci 24:4884-4888.

Mittelsteadt T, Seifert G, Alvarez-Baron E, Steinhauser C, Becker AJ, Schoch S (2009) Differential mRNA expression patterns of the synaptotagmin gene family in the rodent brain. J Comp Neurol 512:514-528.

Murthy VN, Stevens CF (1998) Synaptic vesicles retain their identity through the endocytic cycle. Nature 392:497-501.

Murthy VN, Stevens CF (1999) Reversal of synaptic vesicle docking at central synapses. Nat Neurosci 2:503-507.

Murthy VN, Schikorski T, Stevens CF, Zhu Y (2001) Inactivity produces increases in neurotransmitter release and synapse size. Neuron 32:673-682.

Newton AJ, Kirchhausen T, Murthy VN (2006) Inhibition of dynamin completely blocks compensatory synaptic vesicle endocytosis. Proc Natl Acad Sci U S A 103:1795517960.

Nichols BJ, Lippincott-Schwartz J (2001) Endocytosis without clathrin coats. Trends Cell Biol 11:406-412.

Opazo F, Punge A, Buckers J, Hoopmann P, Kastrup L, Hell SW, Rizzoli SO (2010) Limited Intermixing of Synaptic Vesicle Components upon Vesicle Recycling. Traffic 11:800-812. 
Paddenberg R, Wiegand C, Jockusch BM (1990) Characterization of the coated vesicle uncoating ATPase: tissue distribution, association with and activity on intact coated vesicles. Eur J Cell Biol 52:60-66.

Pang ZP, Sudhof TC (2010) Cell biology of Ca2+-triggered exocytosis. Curr Opin Cell Biol 22:496-505.

Pearse BM (1976) Clathrin: a unique protein associated with intracellular transfer of membrane by coated vesicles. Proc Natl Acad Sci U S A 73:1255-1259.

Perin MS, Fried VA, Mignery GA, Jahn R, Sudhof TC (1990) Phospholipid binding by a synaptic vesicle protein homologous to the regulatory region of protein kinase $\mathrm{C}$. Nature 345:260-263.

Peter BJ, Kent HM, Mills IG, Vallis Y, Butler PJ, Evans PR, McMahon HT (2004) BAR domains as sensors of membrane curvature: the amphiphysin BAR structure. Science 303:495-499.

Pons T, Mattoussi H (2009) Investigating biological processes at the single molecule level using luminescent quantum dots. Ann Biomed Eng 37:1934-1959.

Poskanzer KE, Marek KW, Sweeney ST, Davis GW (2003) Synaptotagmin I is necessary for compensatory synaptic vesicle endocytosis in vivo. Nature 426:559-563.

Poteryaev D, Datta S, Ackema K, Zerial M, Spang A (2010) Identification of the switch in early-to-late endosome transition. Cell 141:497-508.

Prekeris R, Klumperman J, Chen YA, Scheller RH (1998) Syntaxin 13 mediates cycling of plasma membrane proteins via tubulovesicular recycling endosomes. J Cell Biol 143:957-971.

Punge A, Rizzoli SO, Jahn R, Wildanger JD, Meyer L, Schonle A, Kastrup L, Hell SW (2008) $3 \mathrm{D}$ reconstruction of high-resolution STED microscope images. Microsc Res Tech 71:644-650.

Ramjaun AR, McPherson PS (1996) Tissue-specific alternative splicing generates two synaptojanin isoforms with differential membrane binding properties. J Biol Chem 271:24856-24861.

Randall DJ, Burggren WW, Eckert R, French K (2002) Eckert animal physiology : mechanisms and adaptations, Ed. 5. Edition. New York: W.H. Freeman and Co.

Richards DA, Guatimosim C, Betz WJ (2000) Two endocytic recycling routes selectively fill two vesicle pools in frog motor nerve terminals. Neuron 27:551-559.

Rink J, Ghigo E, Kalaidzidis Y, Zerial M (2005) Rab conversion as a mechanism of progression from early to late endosomes. Cell 122:735-749.

Rizzoli SO, Betz WJ (2002) Effects of 2-(4-morpholinyl)-8-phenyl-4H-1-benzopyran-4one on synaptic vesicle cycling at the frog neuromuscular junction. J Neurosci 22:10680-10689.

Rizzoli SO, Betz WJ (2004) The structural organization of the readily releasable pool of synaptic vesicles. Science 303:2037-2039. 
Rizzoli SO, Betz WJ (2005) Synaptic vesicle pools. Nat Rev Neurosci 6:57-69.

Rizzoli SO, Jahn R (2007) Kiss-and-run, collapse and 'readily retrievable' vesicles. Traffic 8:1137-1144.

Rizzoli SO, Bethani I, Zwilling D, Wenzel D, Siddiqui TJ, Brandhorst D, Jahn R (2006) Evidence for early endosome-like fusion of recently endocytosed synaptic vesicles. Traffic 7:1163-1176.

Rosenmund C, Stevens CF (1996) Definition of the readily releasable pool of vesicles at hippocampal synapses. Neuron 16:1197-1207.

Rothman JE (1994) Mechanisms of intracellular protein transport. Nature 372:55-63.

Royle SJ, Lagnado L (2003) Endocytosis at the synaptic terminal. J Physiol 553:345-355.

Rust MJ, Bates M, Zhuang X (2006) Sub-diffraction-limit imaging by stochastic optical reconstruction microscopy (STORM). Nat Methods 3:793-795.

Sankaranarayanan S, De Angelis D, Rothman JE, Ryan TA (2000) The use of pHluorins for optical measurements of presynaptic activity. Biophys J 79:2199-2208.

Sara Y, Virmani T, Deak F, Liu X, Kavalali ET (2005) An isolated pool of vesicles recycles at rest and drives spontaneous neurotransmission. Neuron 45:563-573.

Schiavo G, Stenbeck G, Rothman JE, Sollner TH (1997) Binding of the synaptic vesicle vSNARE, synaptotagmin, to the plasma membrane t-SNARE, SNAP-25, can explain docked vesicles at neurotoxin-treated synapses. Proc Natl Acad Sci U S A 94:9971001.

Schikorski T, Stevens CF (1997) Quantitative ultrastructural analysis of hippocampal excitatory synapses. J Neurosci 17:5858-5867.

Schikorski T, Stevens CF (2001) Morphological correlates of functionally defined synaptic vesicle populations. Nat Neurosci 4:391-395.

Schmidt A, Wolde M, Thiele C, Fest W, Kratzin H, Podtelejnikov AV, Witke W, Huttner WB, Soling HD (1999) Endophilin I mediates synaptic vesicle formation by transfer of arachidonate to lysophosphatidic acid. Nature 401:133-141.

Simons K, van Meer G (1988) Lipid sorting in epithelial cells. Biochemistry 27:61976202.

Simons K, Ikonen E (1997) Functional rafts in cell membranes. Nature 387:569-572.

Simons K, Toomre D (2000) Lipid rafts and signal transduction. Nat Rev Mol Cell Biol 1:31-39.

Smith AM, Wen MM, Nie S (2010) Imaging dynamic cellular events with quantum dots The bright future. Biochem (Lond) 32:12.

Sollner T, Whiteheart SW, Brunner M, Erdjument-Bromage H, Geromanos S, Tempst P, Rothman JE (1993) SNAP receptors implicated in vesicle targeting and fusion. Nature 362:318-324. 
Stenmark H (2009) Rab GTPases as coordinators of vesicle traffic. Nat Rev Mol Cell Biol 10:513-525.

Stenmark H, Olkkonen VM (2001) The Rab GTPase family. Genome Biol 2:REVIEWS3007.

Stenmark H, Parton RG, Steele-Mortimer O, Lutcke A, Gruenberg J, Zerial M (1994) Inhibition of rab5 GTPase activity stimulates membrane fusion in endocytosis. EMBO J 13:1287-1296.

Sudhof TC (2004) The synaptic vesicle cycle. Annu Rev Neurosci 27:509-547.

Südhof TC, Starke K, SpringerLink (Online service) (2008) Pharmacology of Neurotransmitter Release. In: Handbook of Experimental Pharmacology, 184. Berlin, Heidelberg: Springer-Verlag Berlin Heidelberg.

Sun JY, Wu XS, Wu LG (2002) Single and multiple vesicle fusion induce different rates of endocytosis at a central synapse. Nature 417:555-559.

Sutton RB, Fasshauer D, Jahn R, Brunger AT (1998) Crystal structure of a SNARE complex involved in synaptic exocytosis at 2.4 A resolution. Nature 395:347353.

Sweitzer SM, Hinshaw JE (1998) Dynamin undergoes a GTP-dependent conformational change causing vesiculation. Cell 93:1021-1029.

Takamori S et al. (2006) Molecular anatomy of a trafficking organelle. Cell 127:831-846.

Takei K, Mundigl O, Daniell L, De Camilli P (1996) The synaptic vesicle cycle: a single vesicle budding step involving clathrin and dynamin. J Cell Biol 133:1237-1250.

Tansey EM (1997) Not committing barbarisms: Sherrington and the synapse, 1897. Brain Res Bull 44:211-212.

Torri-Tarelli F, Grohovaz F, Fesce R, Ceccarelli B (1985) Temporal coincidence between synaptic vesicle fusion and quantal secretion of acetylcholine. J Cell Biol 101:1386-1399.

Touchot N, Chardin P, Tavitian A (1987) Four additional members of the ras gene superfamily isolated by an oligonucleotide strategy: molecular cloning of YPTrelated cDNAs from a rat brain library. Proc Natl Acad Sci U S A 84:8210-8214.

Valtorta F, Meldolesi J, Fesce R (2001) Synaptic vesicles: is kissing a matter of competence? Trends Cell Biol 11:324-328.

Valtorta F, Jahn R, Fesce R, Greengard P, Ceccarelli B (1988) Synaptophysin (p38) at the frog neuromuscular junction: its incorporation into the axolemma and recycling after intense quantal secretion. J Cell Biol 107:2717-2727.

van den Bogaart G, Holt MG, Bunt G, Riedel D, Wouters FS, Jahn R (2010) One SNARE complex is sufficient for membrane fusion. Nat Struct Mol Biol 17:358-364.

van der Bliek AM, Meyerowitz EM (1991) Dynamin-like protein encoded by the Drosophila shibire gene associated with vesicular traffic. Nature 351:411-414. 
Voglmaier SM, Kam K, Yang H, Fortin DL, Hua Z, Nicoll RA, Edwards RH (2006) Distinct endocytic pathways control the rate and extent of synaptic vesicle protein recycling. Neuron 51:71-84.

Walther K, Diril MK, Jung N, Haucke V (2004) Functional dissection of the interactions of stonin 2 with the adaptor complex AP-2 and synaptotagmin. Proc Natl Acad Sci U S A 101:964-969.

Weimbs T, Low SH, Chapin SJ, Mostov KE, Bucher P, Hofmann K (1997) A conserved domain is present in different families of vesicular fusion proteins: a new superfamily. Proc Natl Acad Sci U S A 94:3046-3051.

Westphal V, Rizzoli SO, Lauterbach MA, Kamin D, Jahn R, Hell SW (2008) Video-rate farfield optical nanoscopy dissects synaptic vesicle movement. Science 320:246249.

Wienisch M, Klingauf J (2006) Vesicular proteins exocytosed and subsequently retrieved by compensatory endocytosis are nonidentical. Nat Neurosci 9:1019-1027.

Wildanger D, Rittweger E, Kastrup L, Hell SW (2008) STED microscopy with a supercontinuum laser source. Opt Express 16:9614-9621.

Willig KI, Rizzoli SO, Westphal V, Jahn R, Hell SW (2006) STED microscopy reveals that synaptotagmin remains clustered after synaptic vesicle exocytosis. Nature 440:935-939.

Wu W, Xu J, Wu XS, Wu LG (2005) Activity-dependent acceleration of endocytosis at a central synapse. J Neurosci 25:11676-11683.

Wucherpfennig T, Wilsch-Brauninger M, Gonzalez-Gaitan M (2003) Role of Drosophila Rab5 during endosomal trafficking at the synapse and evoked neurotransmitter release. J Cell Biol 161:609-624.

Xia Z, Dudek H, Miranti CK, Greenberg ME (1996) Calcium influx via the NMDA receptor induces immediate early gene transcription by a MAP kinase/ERK-dependent mechanism. J Neurosci 16:5425-5436.

Yamamoto T, Davis CG, Brown MS, Schneider WJ, Casey ML, Goldstein JL, Russell DW (1984) The human LDL receptor: a cysteine-rich protein with multiple Alu sequences in its mRNA. Cell 39:27-38.

Zenisek D, Steyer JA, Feldman ME, Almers W (2002) A membrane marker leaves synaptic vesicles in milliseconds after exocytosis in retinal bipolar cells. Neuron 35:1085-1097.

Zhang Q, Li Y, Tsien RW (2009) The dynamic control of kiss-and-run and vesicular reuse probed with single nanoparticles. Science 323:1448-1453. 


\section{A. ApPEnDIX}

\section{Matlab Routines for pHluorin Analysis}

Phluorin analysis was largely performed as described (Mitchell and Ryan, 2004). Briefly, boutonal areas increasing in fluorescence during stimulation were automatically identified as active boutons for analysis. The boutonal fluorescence was then calculated for each frame as the difference compared to the initial (pre-stimulation) fluorescence $(d F)$. This $d F$ was normalized by dividing by the initial fluorescence, yielding $d F / F_{0}$. The routines used for pHlorin data analysis are summarized in Table A-1, and listed below.

\section{Table A-1: Matlab functions used for pHluorin analysis}

\begin{tabular}{l|l} 
Function & Purpose of the function \\
\hline pHluorin_analysis & main program; reads image files and calls 'autoselection' \\
\hline autoselection & shows movie data; asks for selection of background and bad areas \\
\hline pHgetline_0 & detects the type of mouse click (normal or shift+click) \\
\hline pHgetline_1; pHgetline_22 & allow marking of background areas \\
\hline pHgetline_1_b; pHgetline_22_b & allow marking of bad areas to be excluded \\
\hline diffview_auto2; pHluorin_addFrames & thresholding of movies and calculation of dF plots \\
\hline p_quit_auto_final & final data processing and saving of results
\end{tabular}

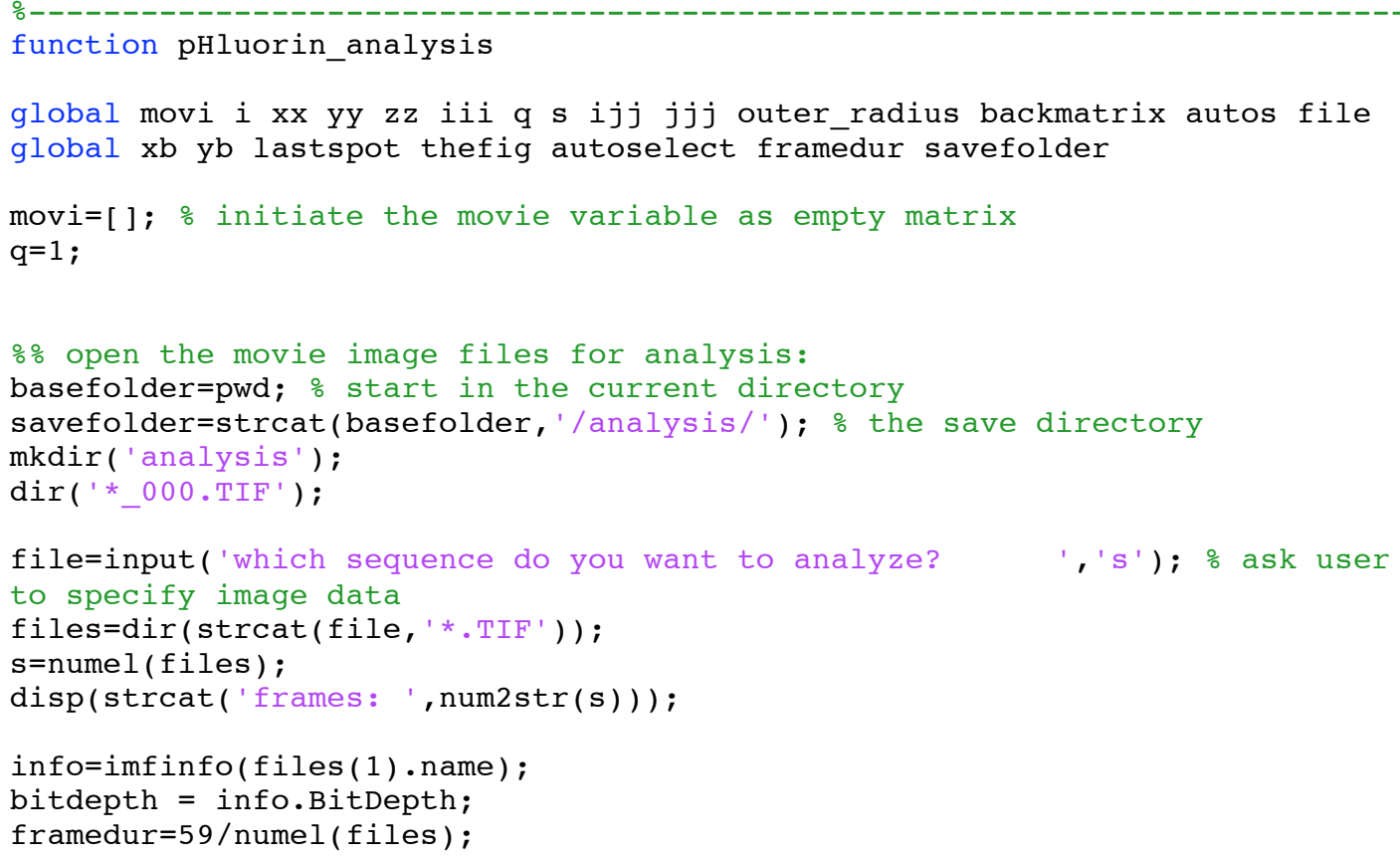




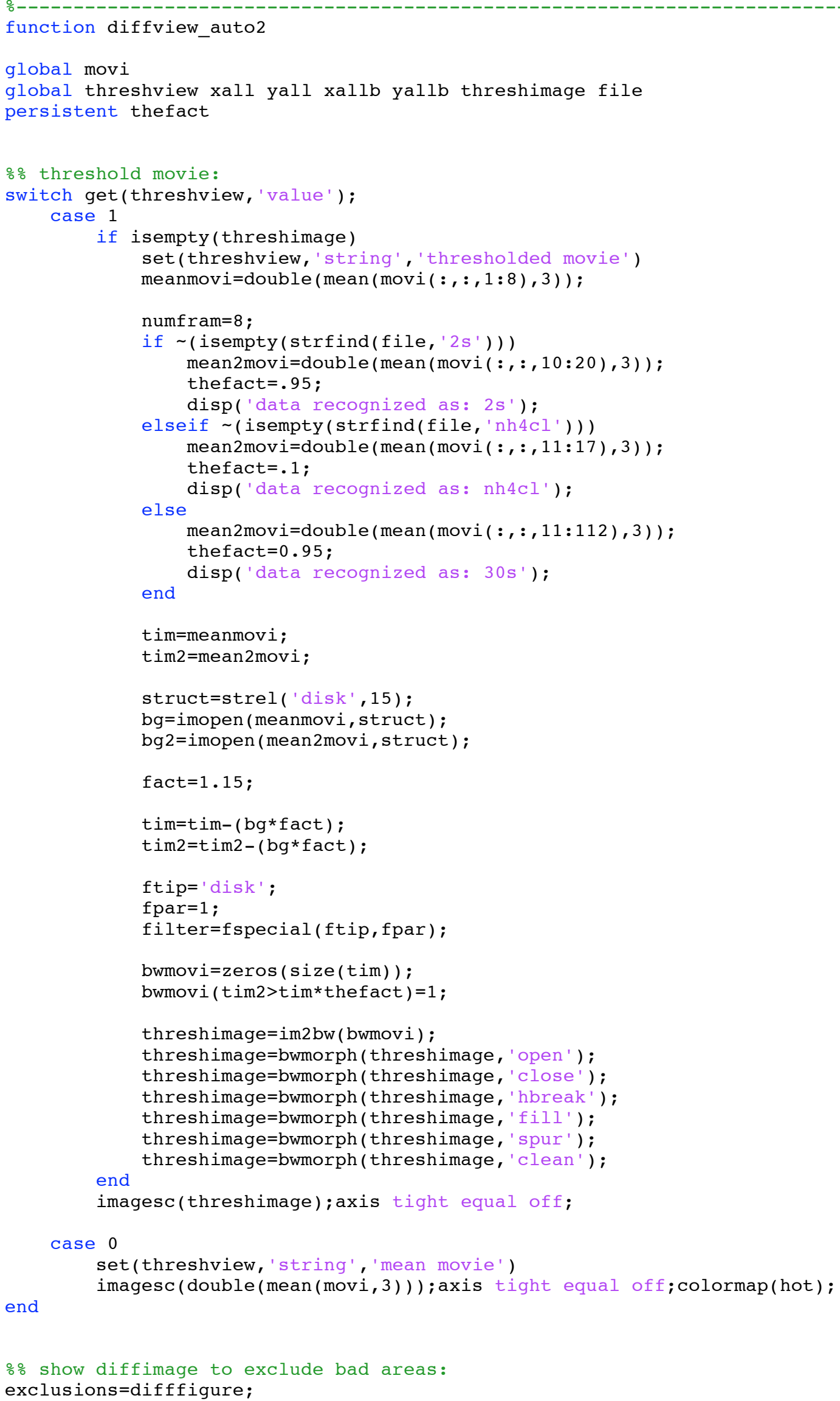




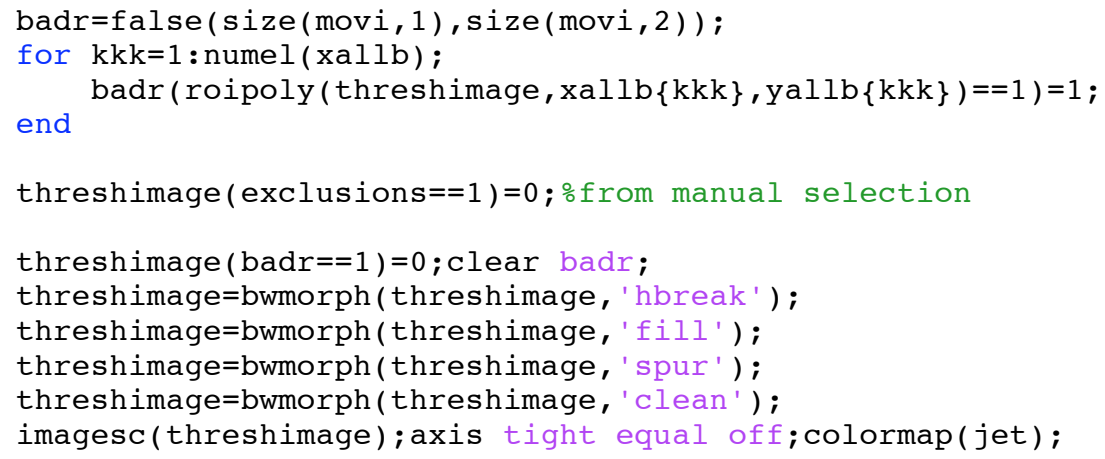




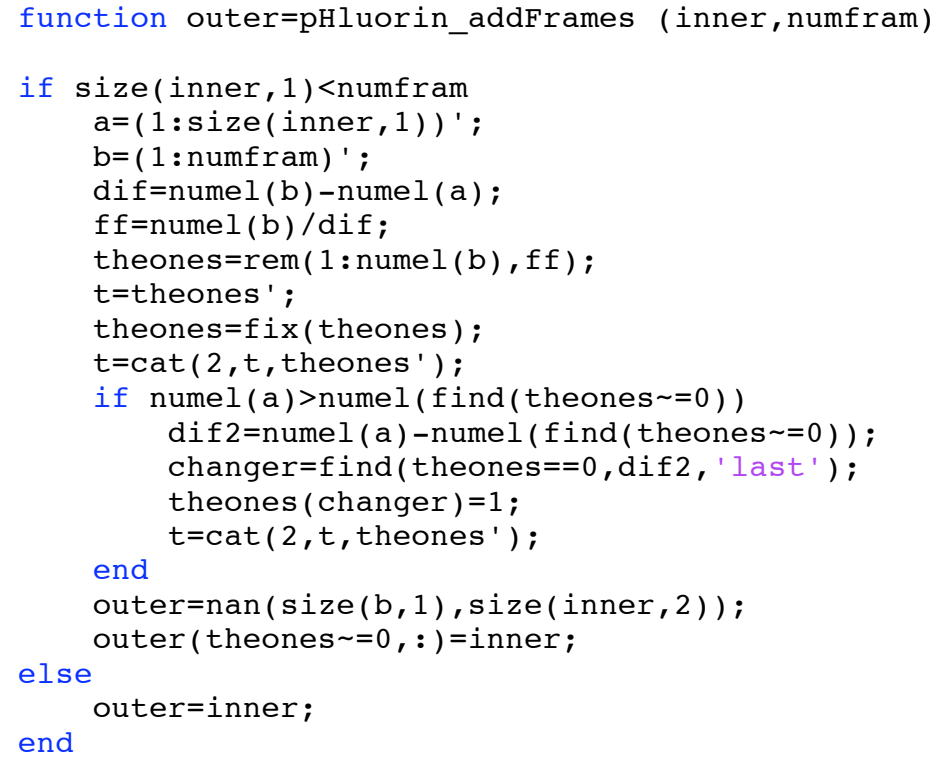




\section{Matlab Routines for in vitro Immunoassay Analysis}

The isolated synaptic vesicle immunoassay experiments were performed and analyzed as described in section 2.12 (and as established in detail in Hoopmann, 2008). The Matlab routines used for analysis are summarized in , and listed below.

Table A-2: Matlab functions used for analysis of isolated vesicle immunoassays

\begin{tabular}{l|l} 
Function & Purpose of the function \\
\hline AutoPHluoro & main program; reads images and calls 'AutoAlignImages' \\
\hline AutoAlignImages & aligns the images based the location of the beads \\
\hline pH_spotfinda_wgr & filters images and locates the spots in all channels \\
\hline pH_gp_catmonopoly_GetThresh & $\begin{array}{l}\text { gets threshold for the exclusion of background, which is observed } \\
\text { in absence of vesicles }\end{array}$ \\
\hline pH_findtritccyf_genpool & calculates the fractions of vesicles for the general pool \\
\hline pH_findtritccyf_tritcclick & calculates the fractions of vesicles for the recycling pool
\end{tabular}

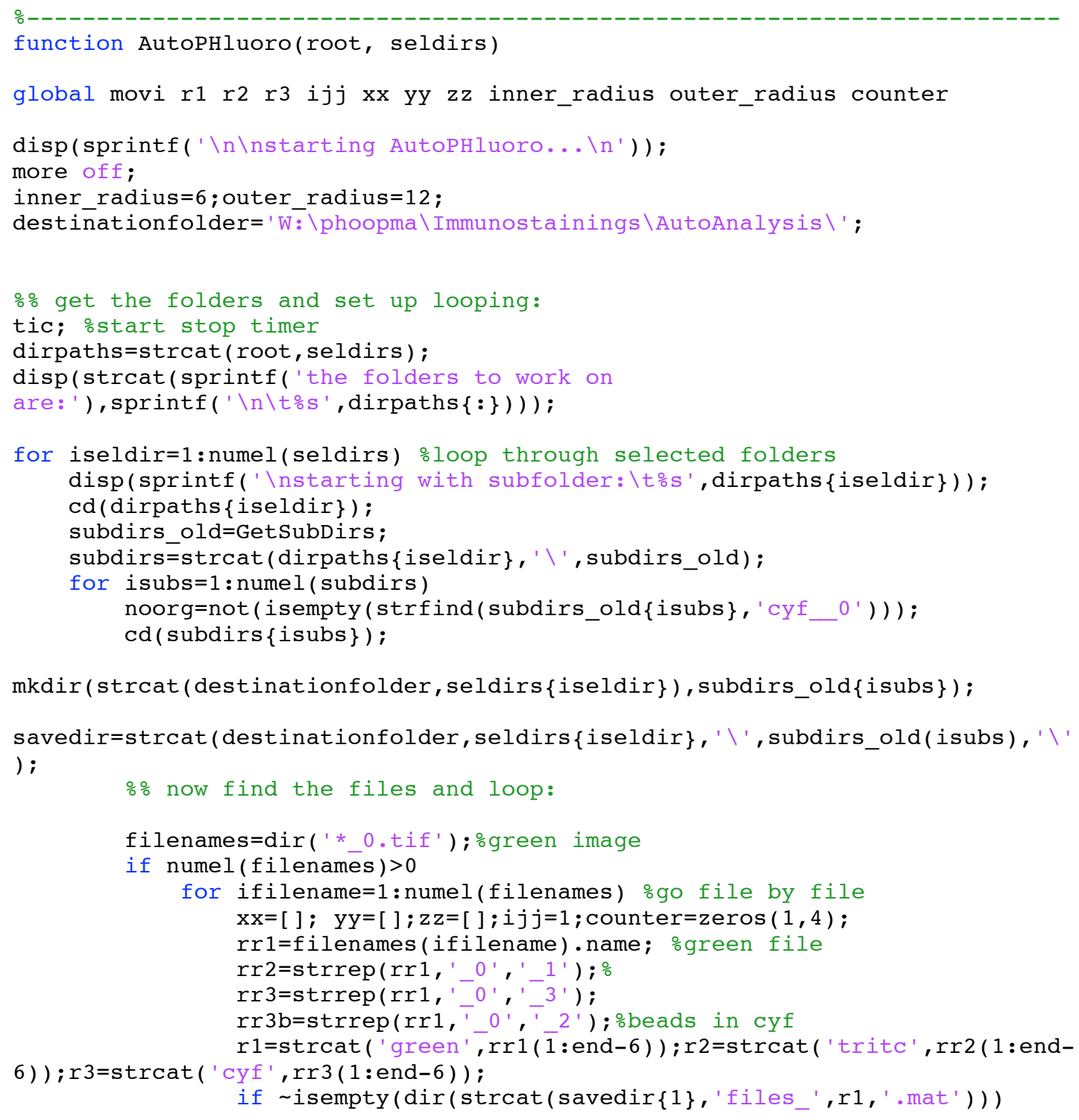




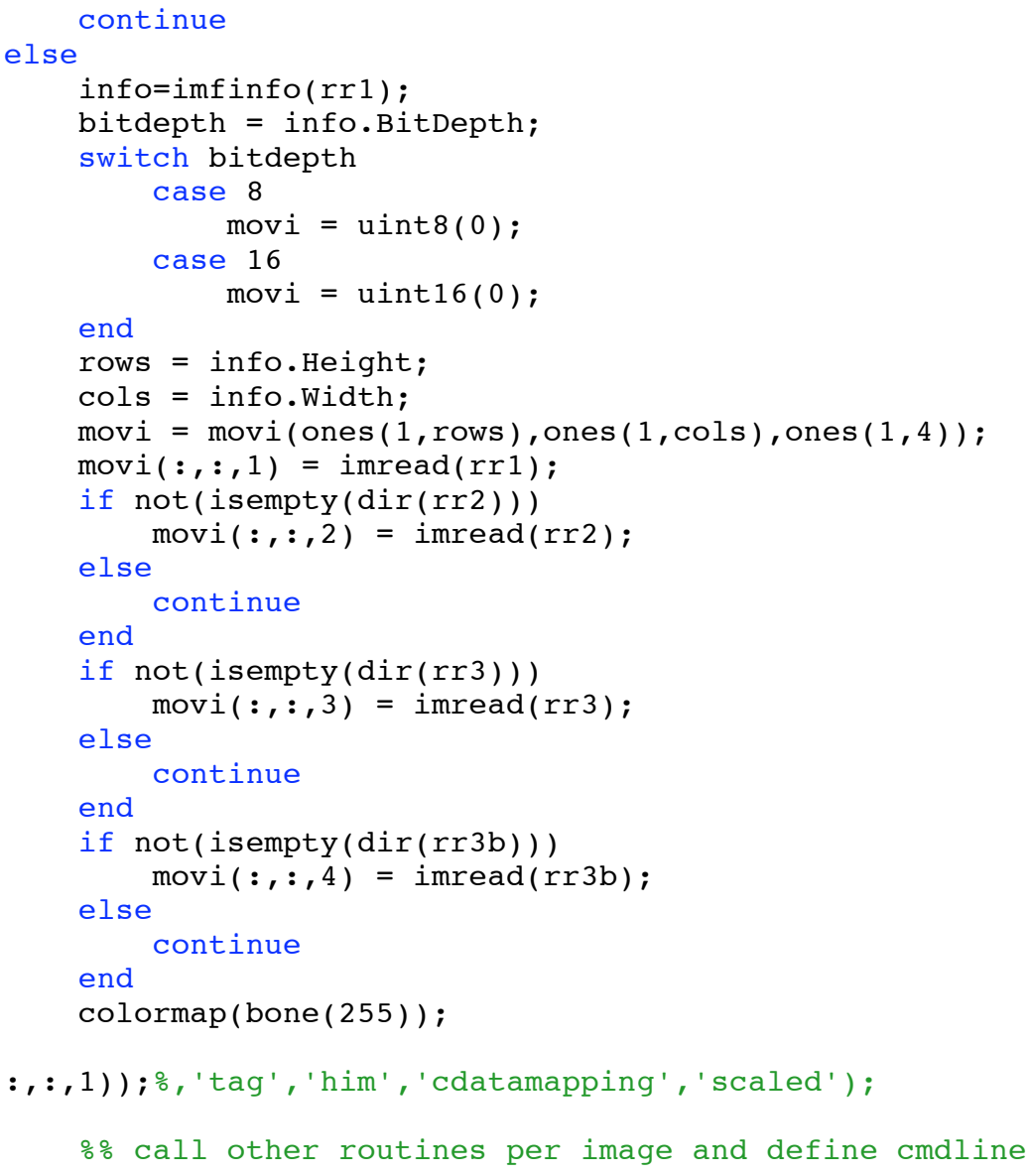


\% $\frac{\circ}{\circ}$ ind the beads

bw_green=movi $(:,:, 1)$;

bw_green (find (bw_green<=prctile $(\operatorname{prctile}(\operatorname{double}(\operatorname{movi}(:,:, 1)), 98.95), 98.95))$ ) =

0 ;

bw_green=logical (bw_green);

bw_tritc=movi $(:,:, 2)$;

bw_tritc (find (bw_tritc $<=\operatorname{prctile}(\operatorname{prctile}(\operatorname{double}(\operatorname{movi}(:, \mathbf{a}, 2)), 99.25), 99.25)))=$

0 ;

bw_tritc=logical (bw_tritc);

bw_cyf=movi $(:,:, 4)$;

bw_cyf (find (bw_cyf<=prctile (prctile (double (movi $(:,:, 4)), 99.35), 99.35)$ )) =0 ;

bw_cyf=logical (bw_cyf);

bw_green=bwmorph (bw_green, 'clean');

bw_tritc=bwmorph (bw_tritc, 'clean');

bw_cyf=bwmorph (bw_cyf , 'clean');

$\% \%$

[green_labeled,green_numobj] = bwlabel(bw_green,4);clear bw_green;

bw_greēn_data = regiōnprops (green_labeled,' centroid');clear green_labeled;

[tritc_labeled,tritc_numobj] = bwlabel(bw_tritc,4);clear bw_tritc;

bw_trit̄c_data = regiōonprops (tritc_labeled,' centroid');clear tritc_labeled;

[cyf_labeled,cyf_numobj] = bwlabel(bw_cyf,4);clear bw_cyf;

bw_cyf_data = reḡionprops (cyf_labeled,' centroid'); cleār cyf_labeled;

if tritc_numobj $>50$

disp(sprintf('continuing without aligning...(tritc >50)' ));

pH_spotfinda_wgr([ ], [ ], [ ], [ ], imagesize, savedir, noorg,minint_tritc,minint_cyf , minint_gr, memin, megremin);

clear dist*

bw*;xx_bead=[ ] ;yy_bead=[ ];xx_gr=[ ] ;yy_gr=[ ]; xx_cyf=[ ];yy_cyf=[ ];distx=[ ];dis ty $=[$ ] return

elseif tritc numobj==0

disp(sprintf('continuing without aligning...'));

pH_spotfinda_wgr([], [ ], [ ], [ ], imagesize, savedir, noorg,minint_tritc,minint_cyf , minint_gr, memin, megremin);

clear dist*

bW*;xx_bead=[ ] ;yy_bead=[ ];xx_gr=[ ] ;yy_gr=[ ];xx_cyf=[ ];yy_cyf=[ ];distx=[ ];dis ty $=[$ ]

return

else

xx_bead=zeros $(1$, tritc_numobj) ;yy_bead=zeros $(1$, tritc_numobj ) ;

for $i=1:$ tritc numobj

$x x$ bead $\left(i \overline{)}=b w \_t r i t c \_d a t a(i) \cdot\right.$ Centroid ( 1$)$;

end

yy_bead (i)=bw_tritc_data (i) . Centroid (2);

end

clear bw tritc data;

if green_numobj $>350$

BlackBBeads (imagesize, xx_bead,yy_bead);

disp(sprintf('continuing without aligning...(green >350)'));

pH_spotfinda_wgr([ ] , [ ] , xx_bead,yy_bead, imagesize, savedir, noorg,minint_tritc, min̄int cyf, minint gr, memin̄, megremin);

clēer dist*

bw*;xx_bead=[ ] ;yy_bead=[ ] ; xx_gr=[ ] ;yy_gr=[ ]; xx_cyf=[ ];yy_cyf=[ ];distx=[ ];dis

ty $=[$ ]

return

elseif green_numobj= $=0$

BlackBeads (imagesize, $x x$ bead, yy bead);

disp(sprintf('continuing without aligning...')); 


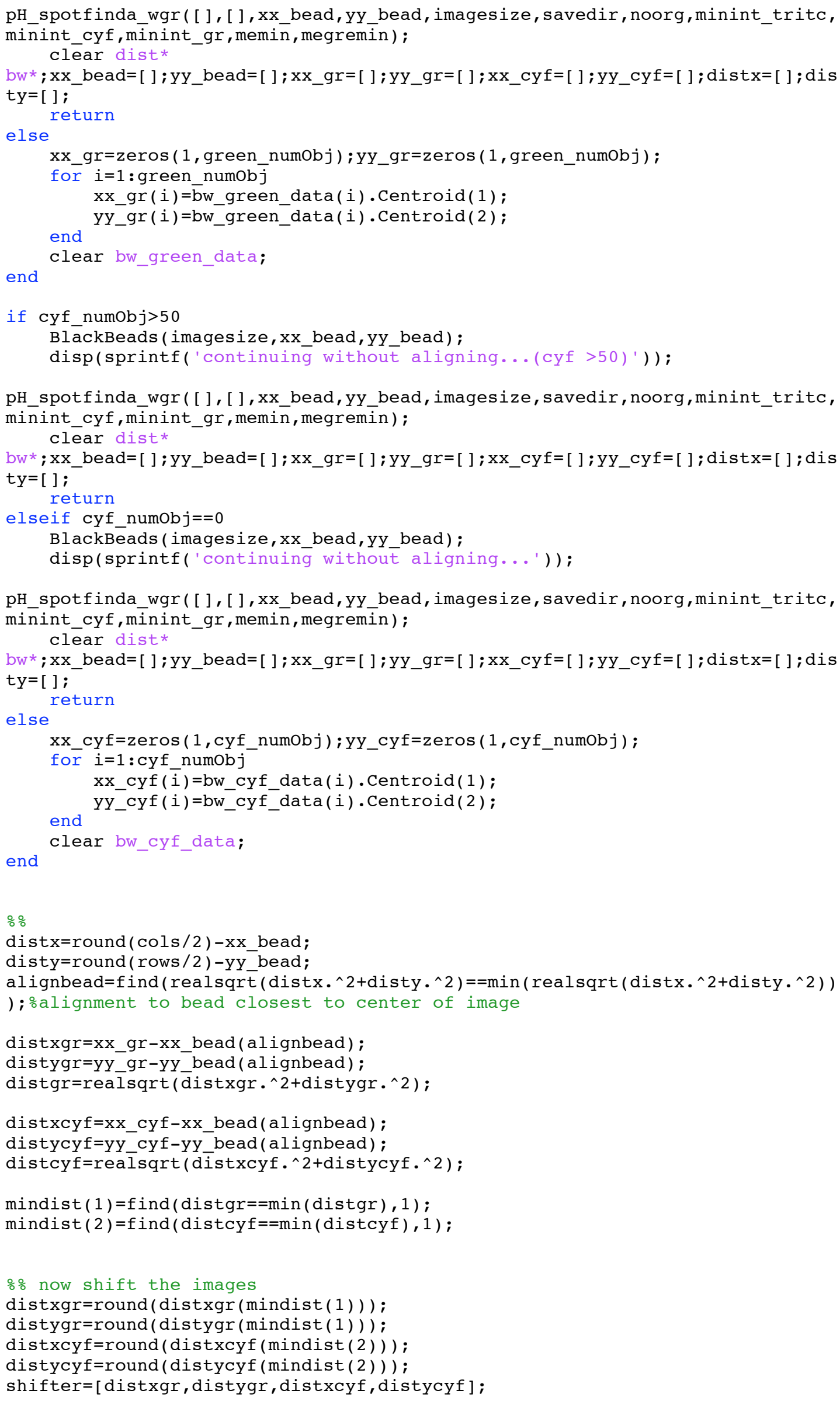

pH_spotfinda_wgr([ ] , [ ] , xx_bead,yy_bead,imagesize, savedir, noorg,minint_tritc , minint cyf, minint gr, memin, megremin) ; 


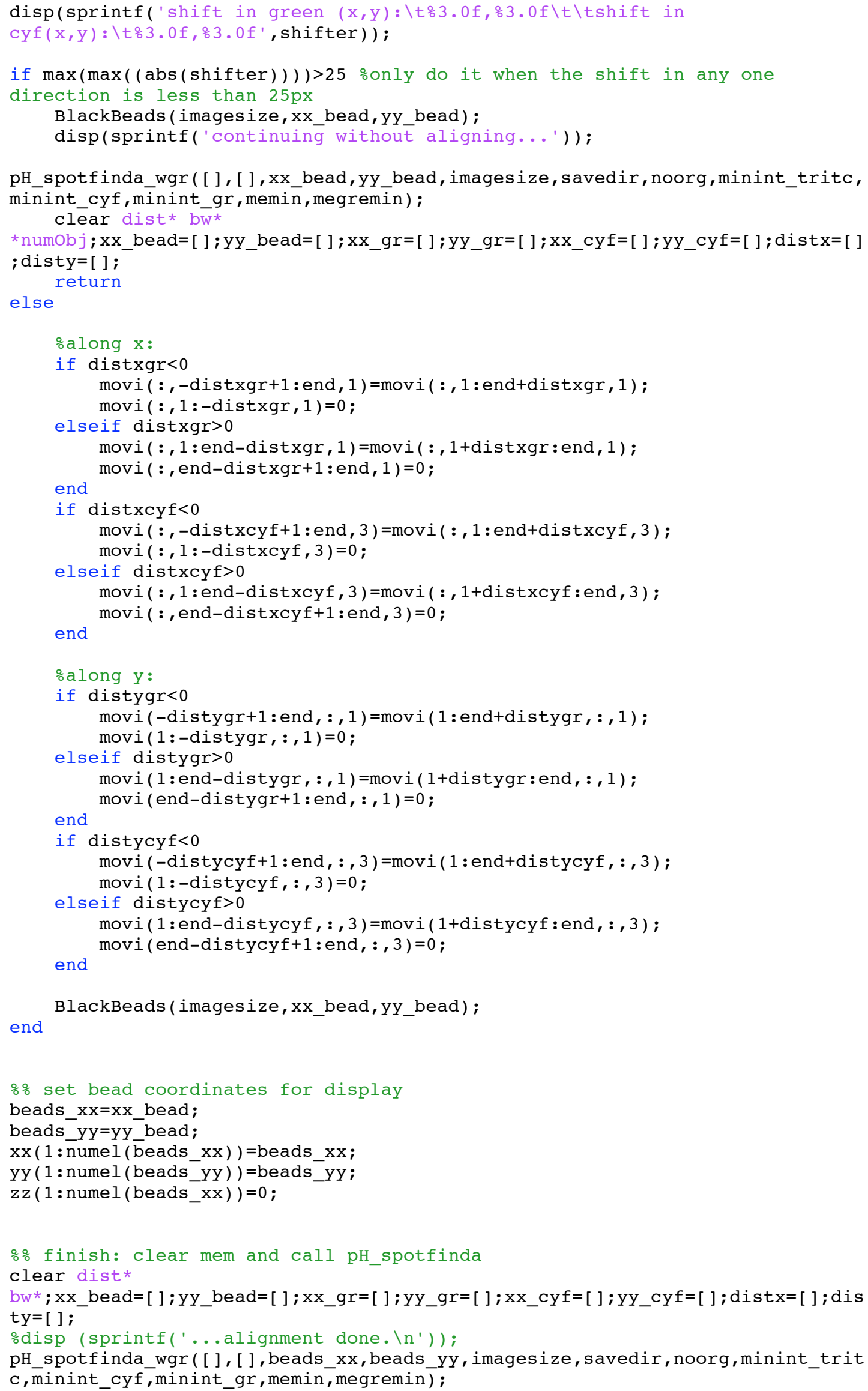




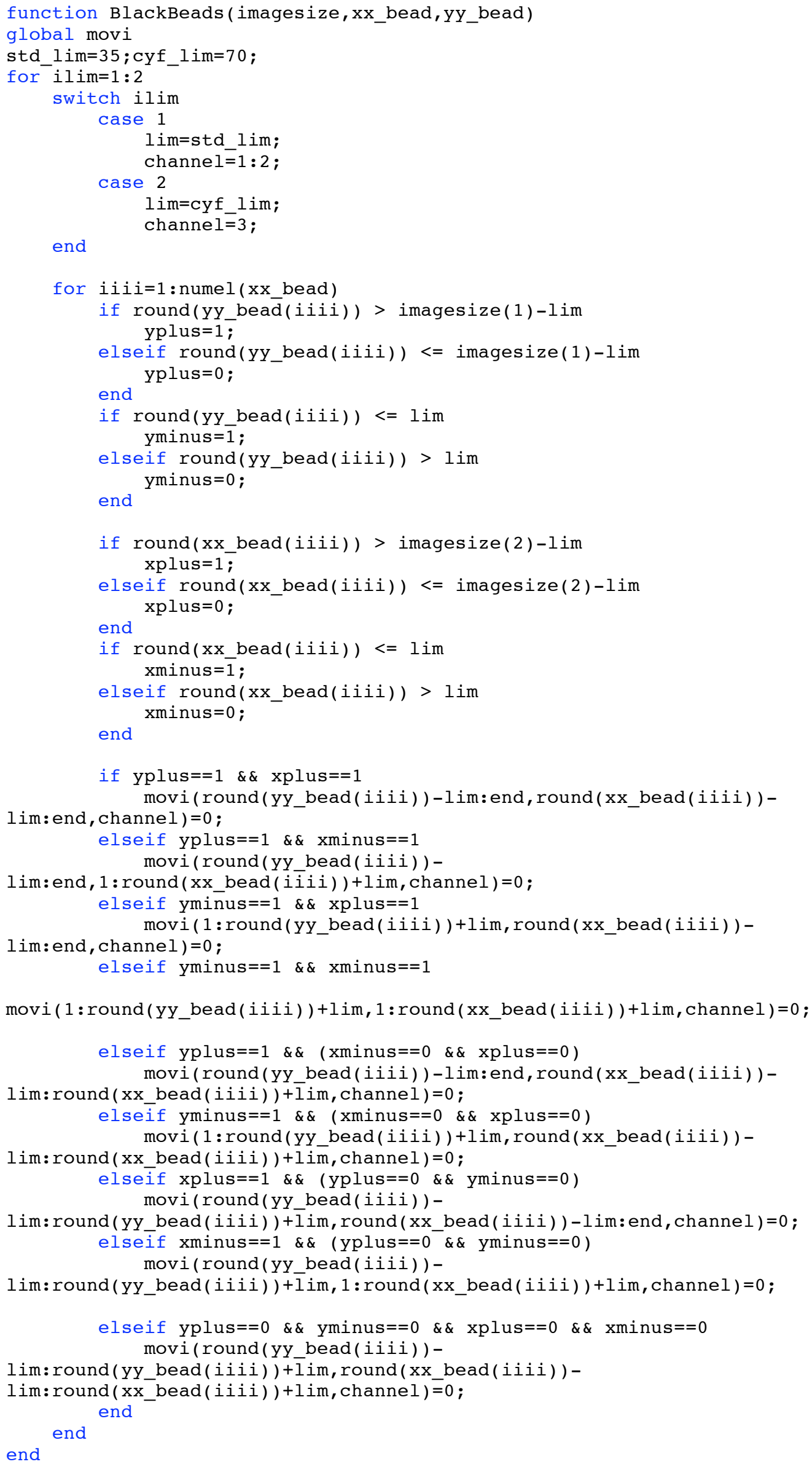




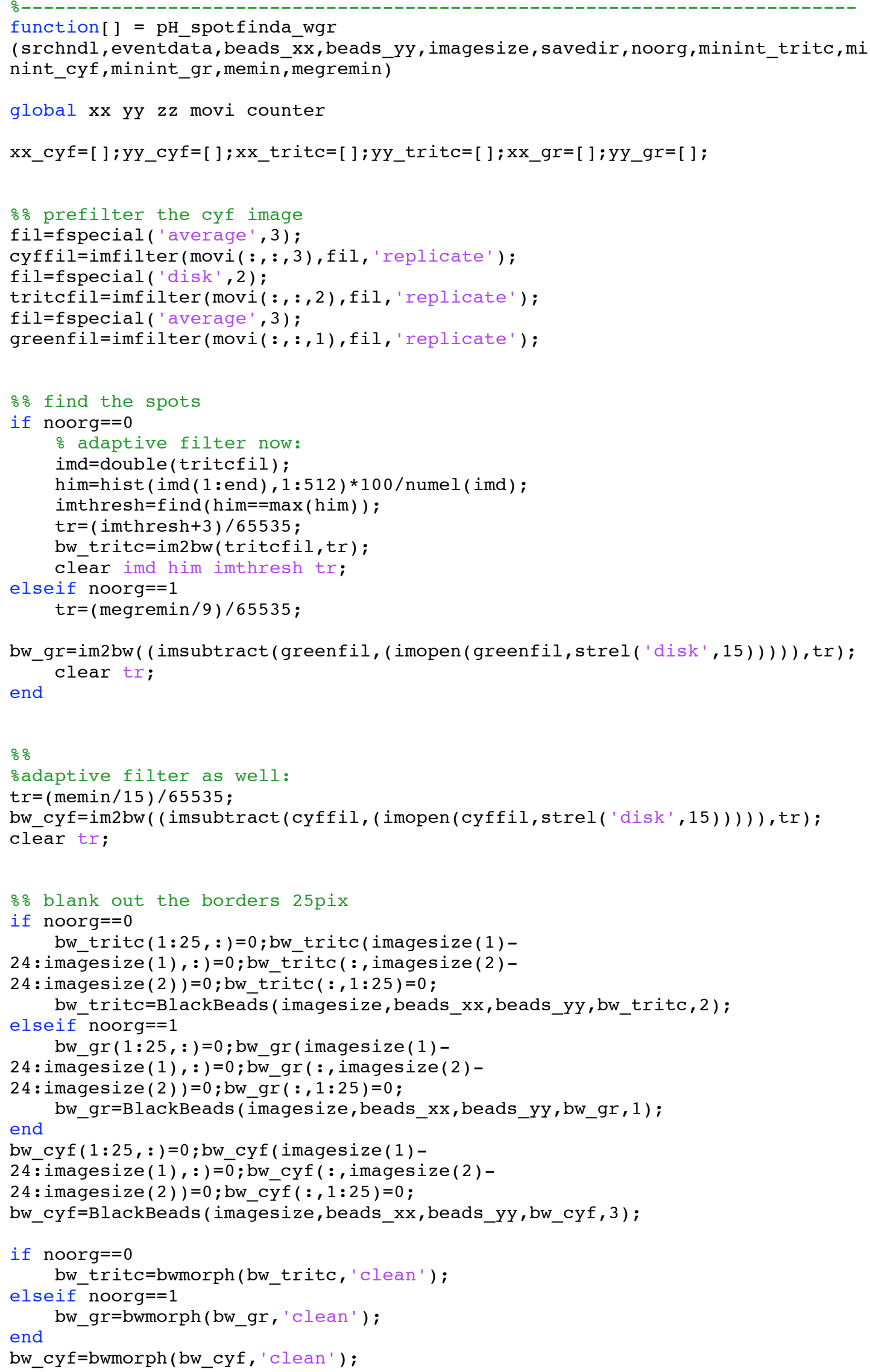




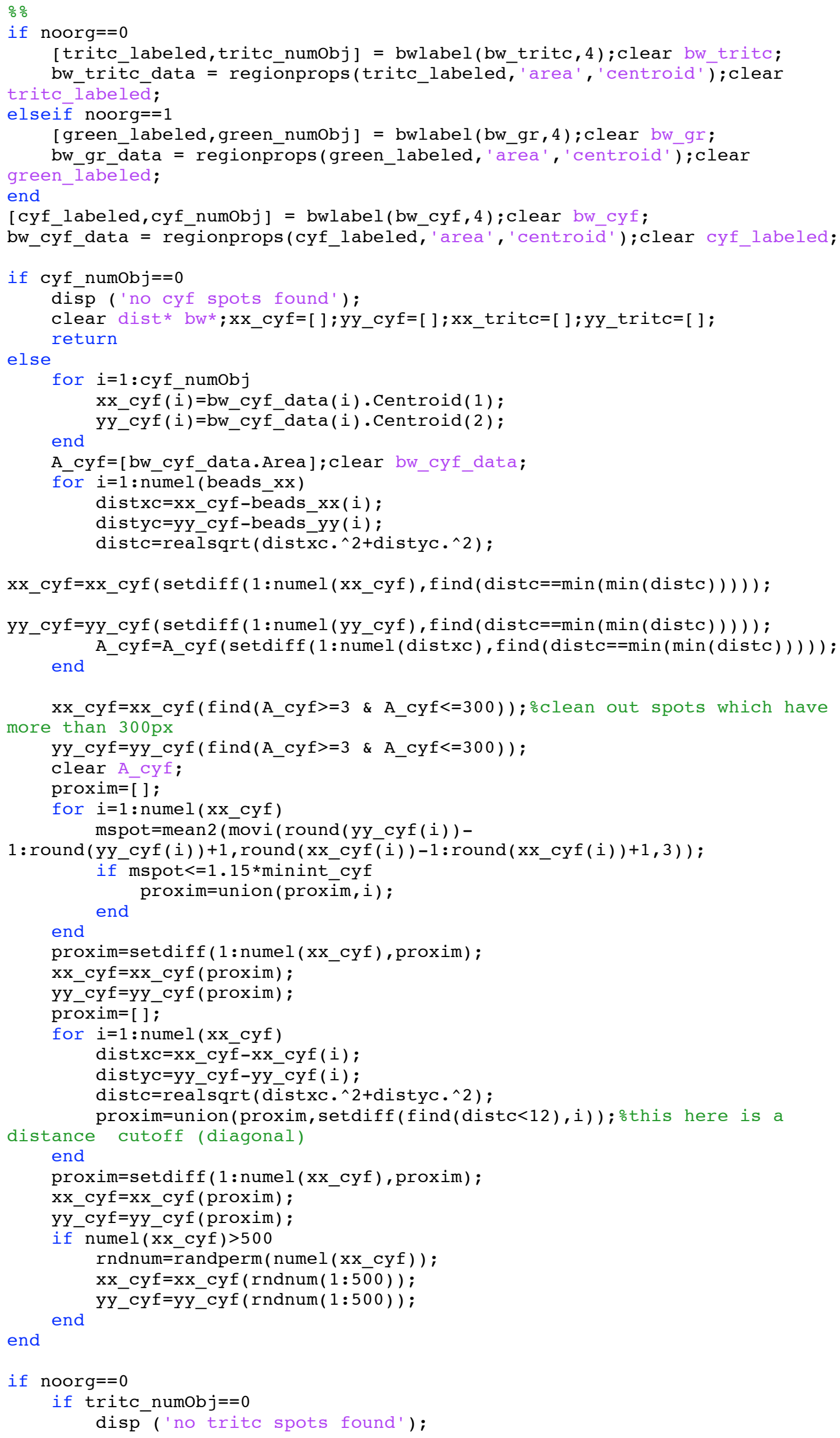




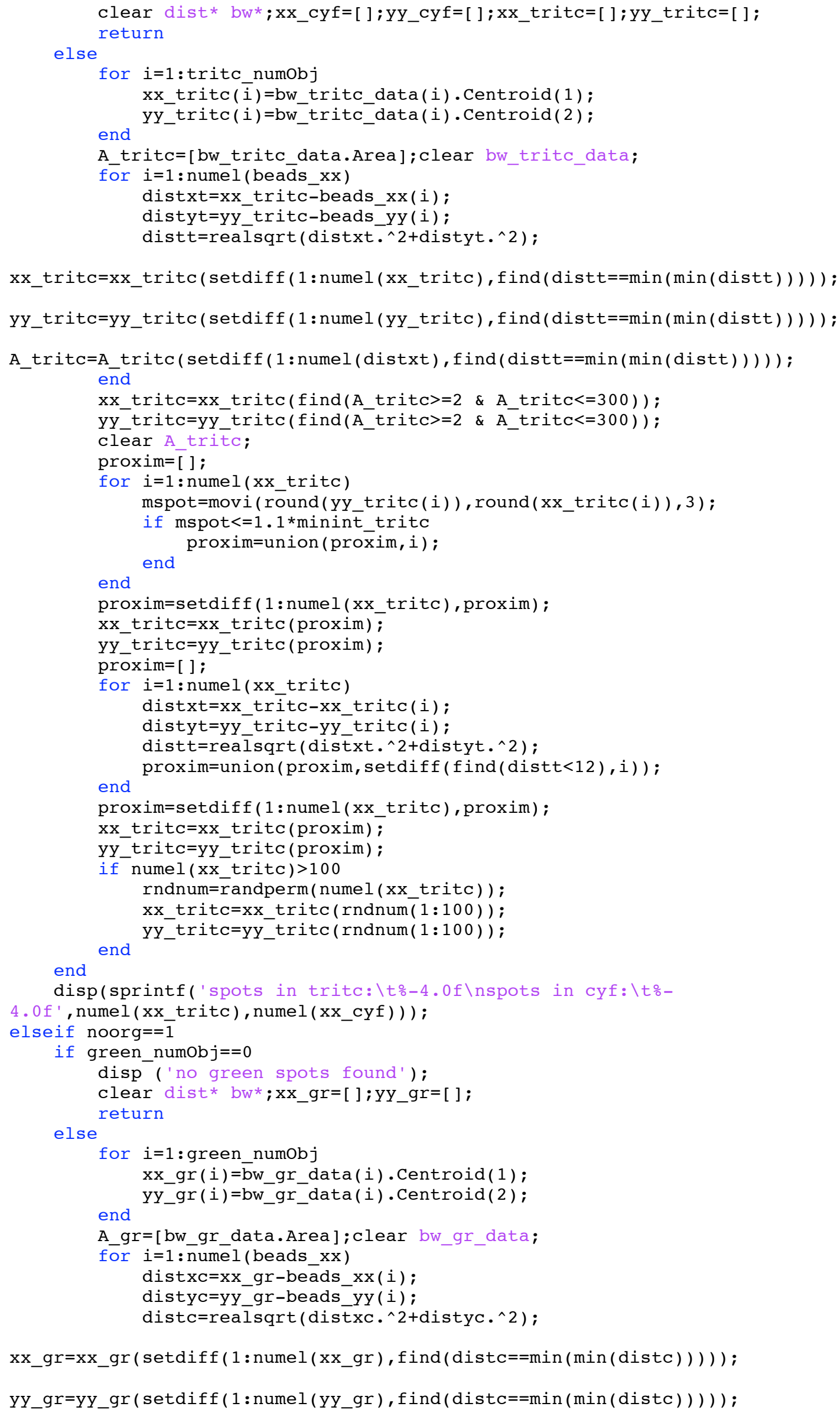




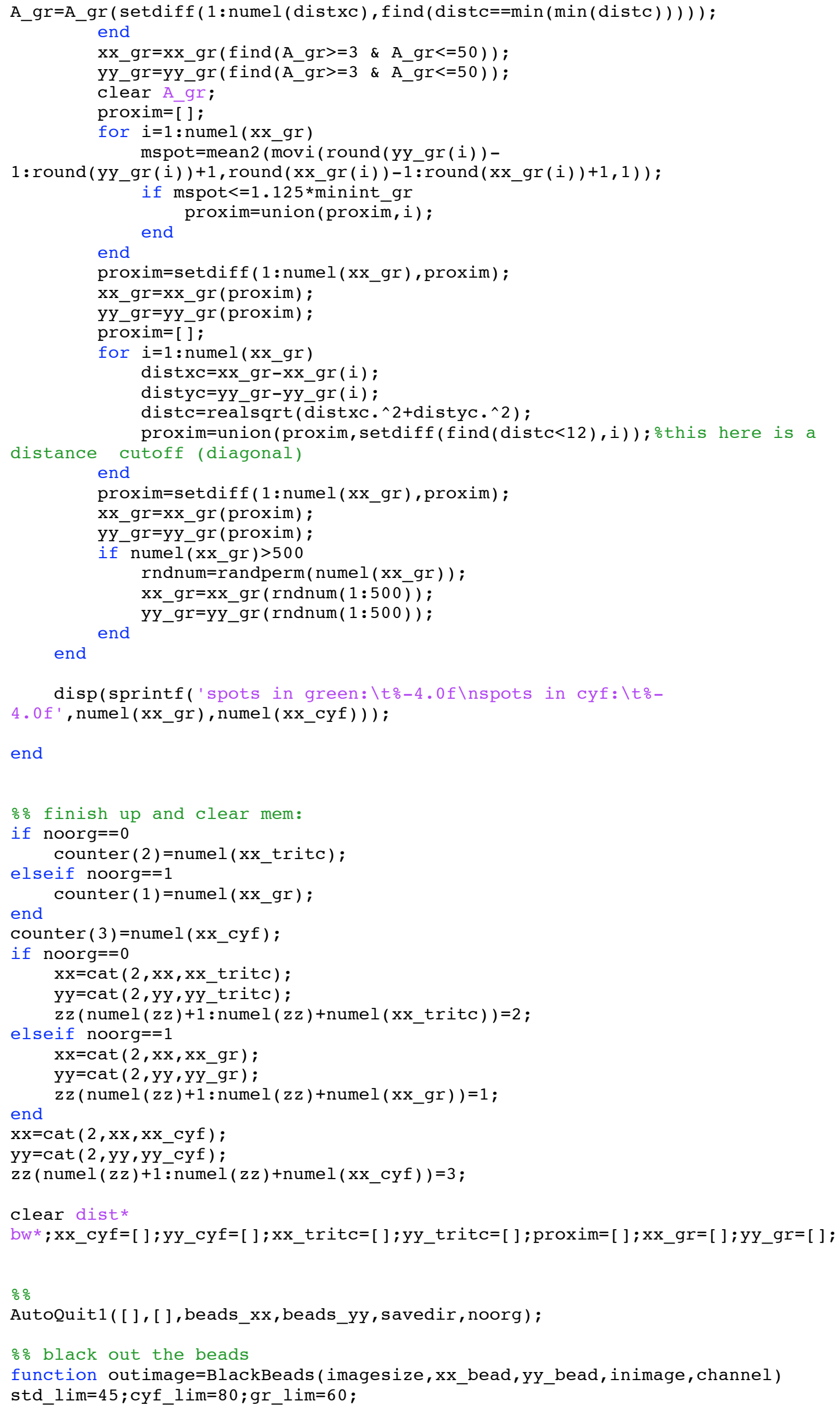




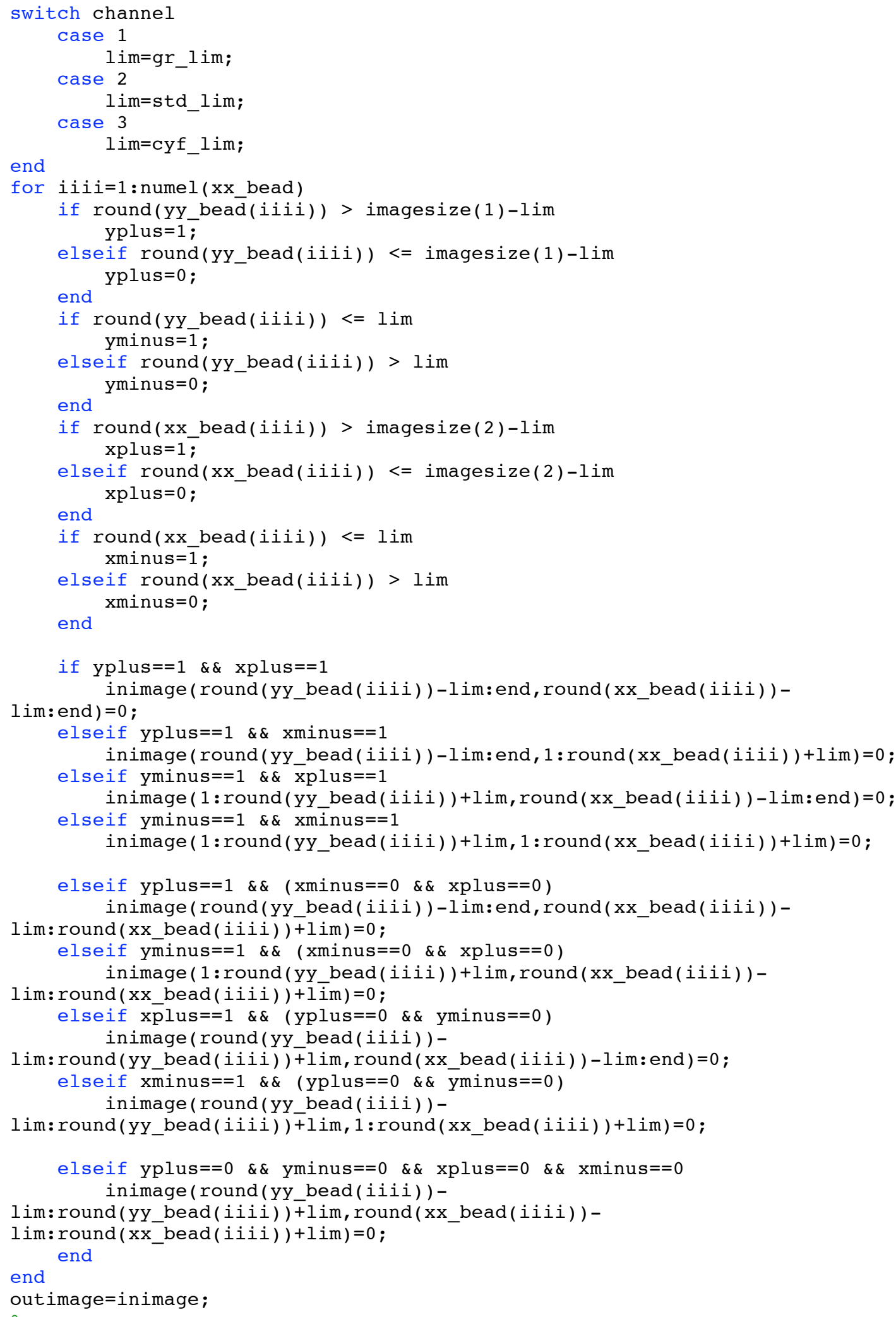




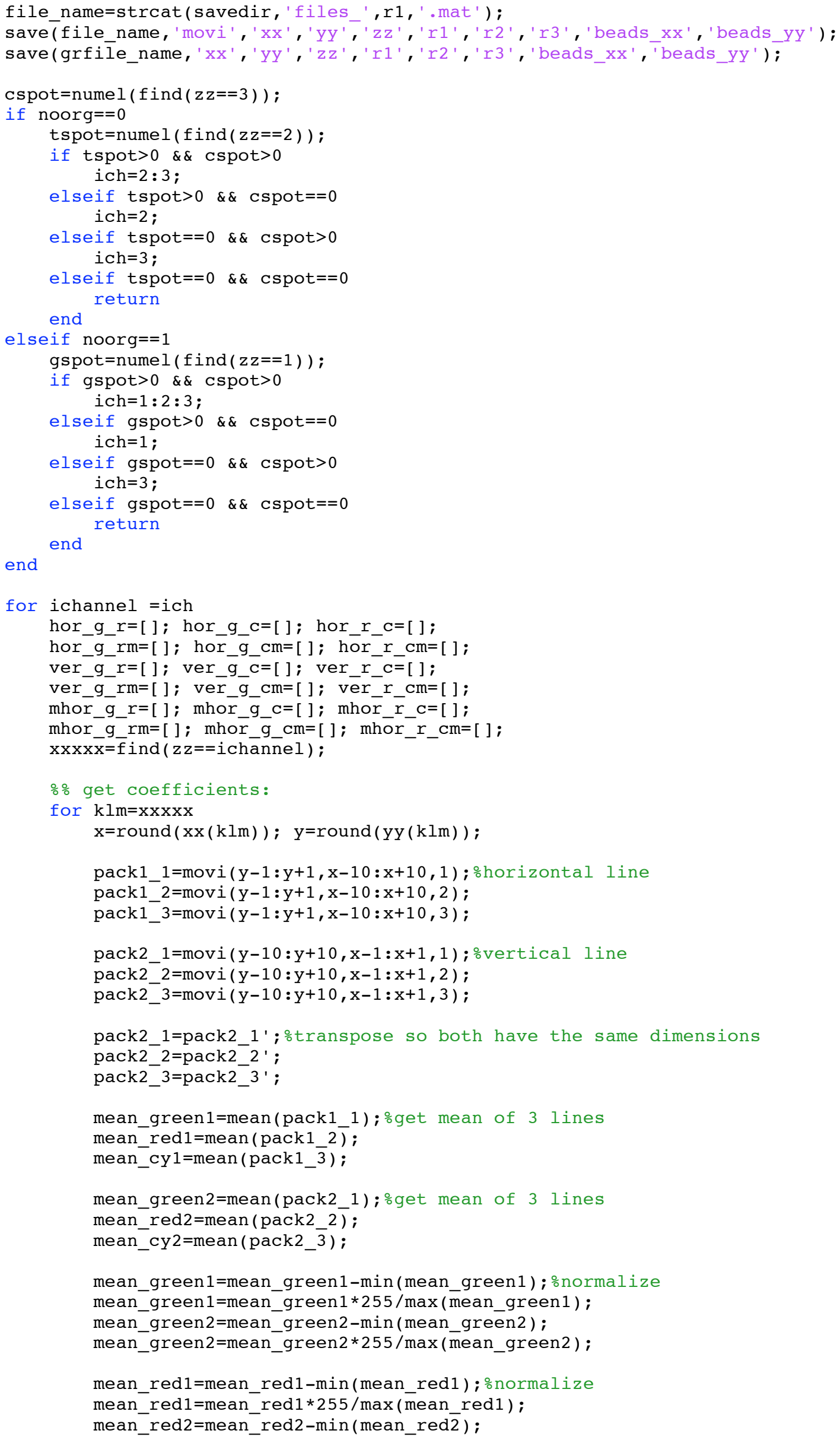




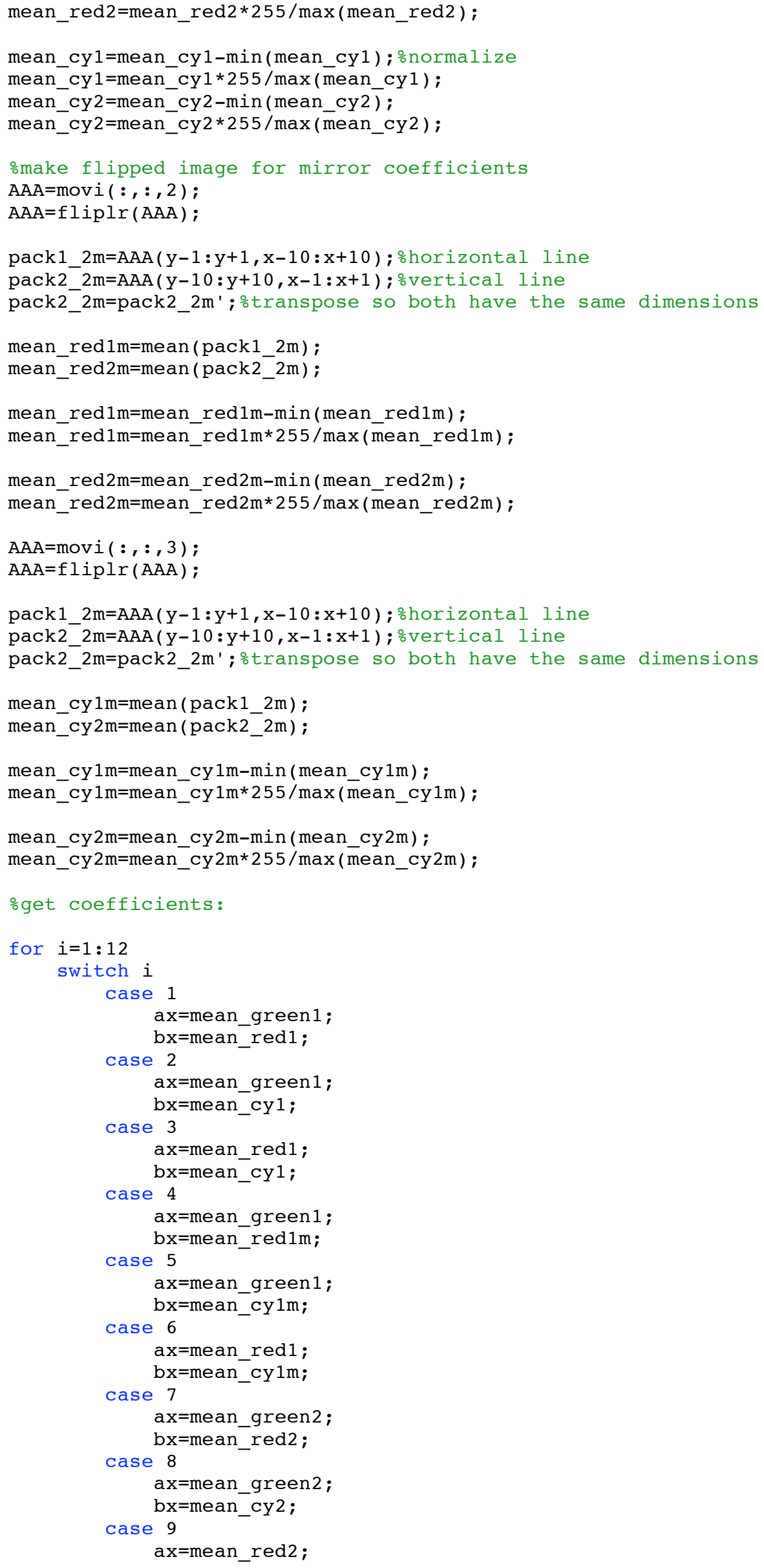




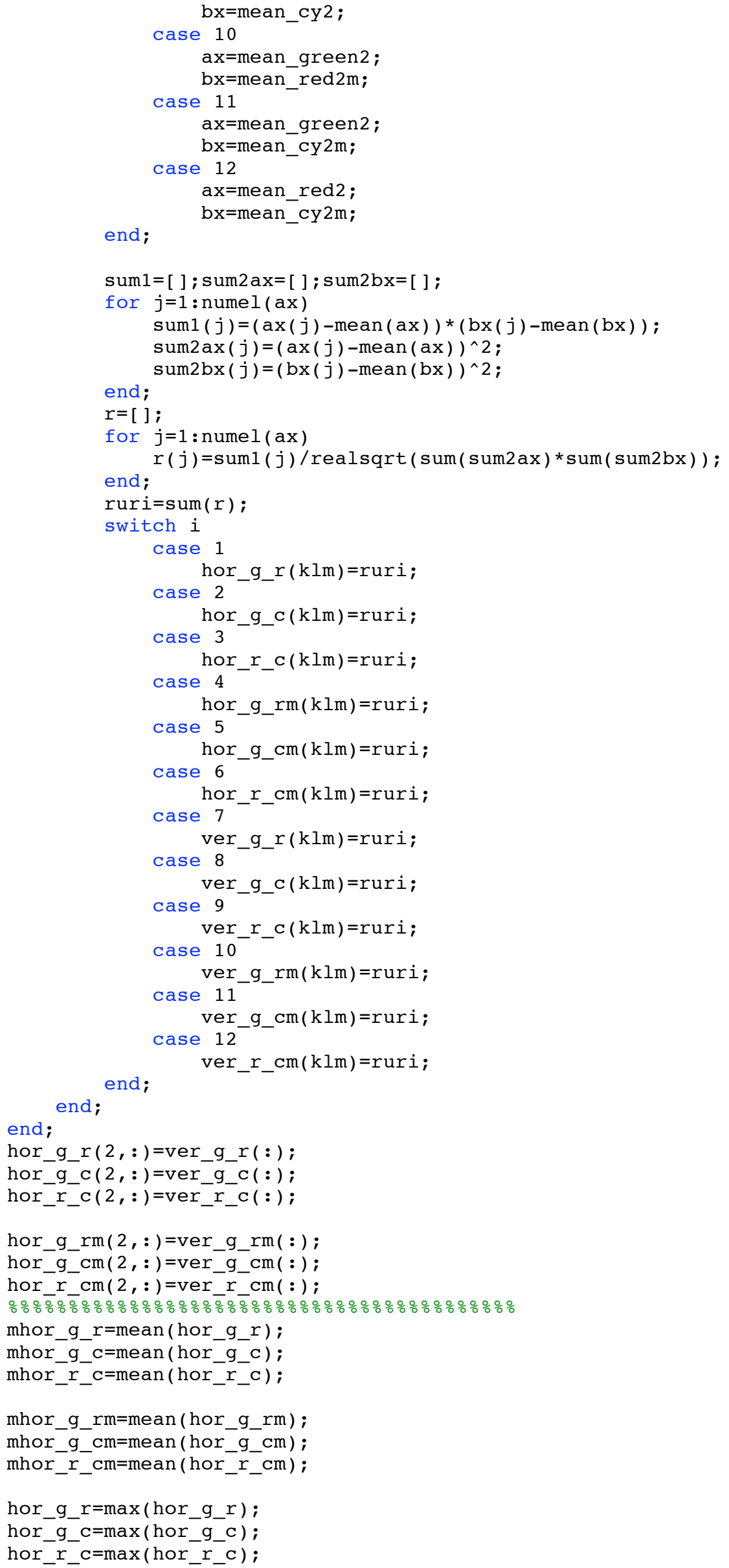




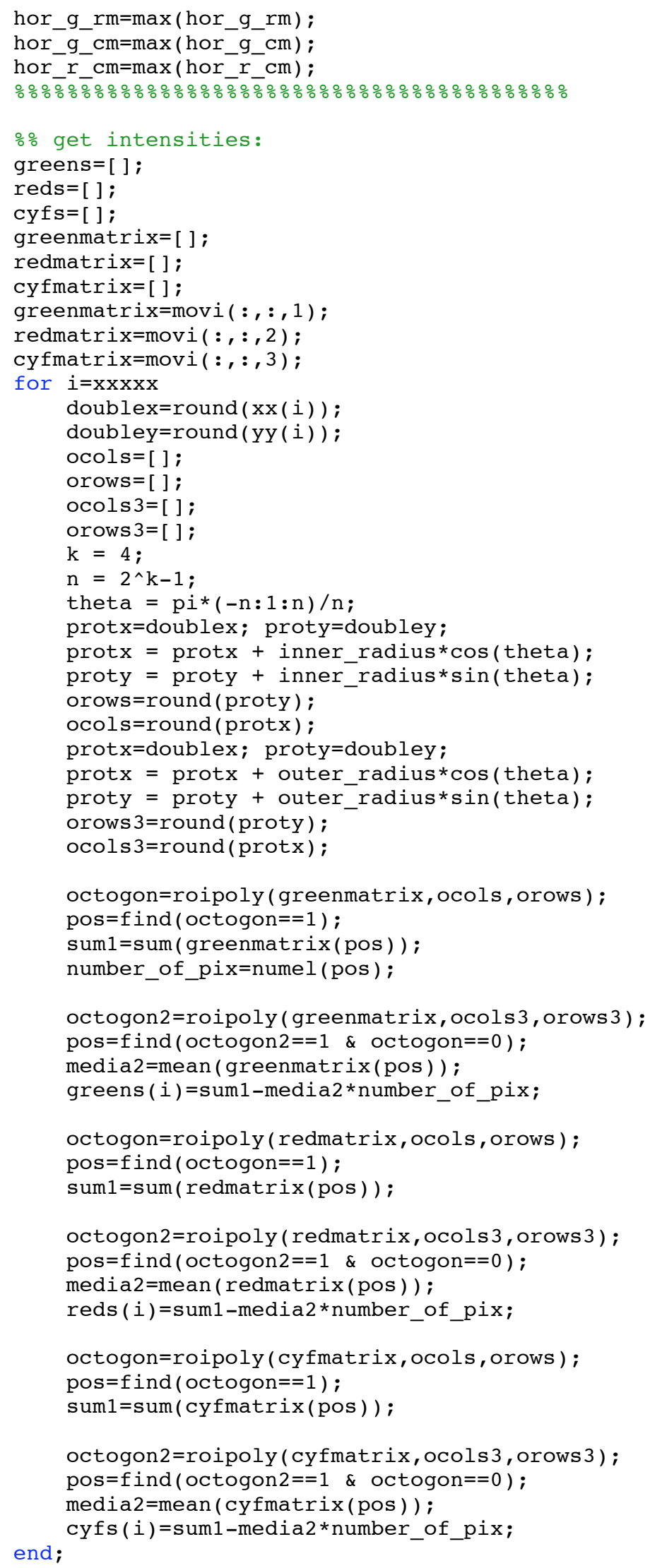




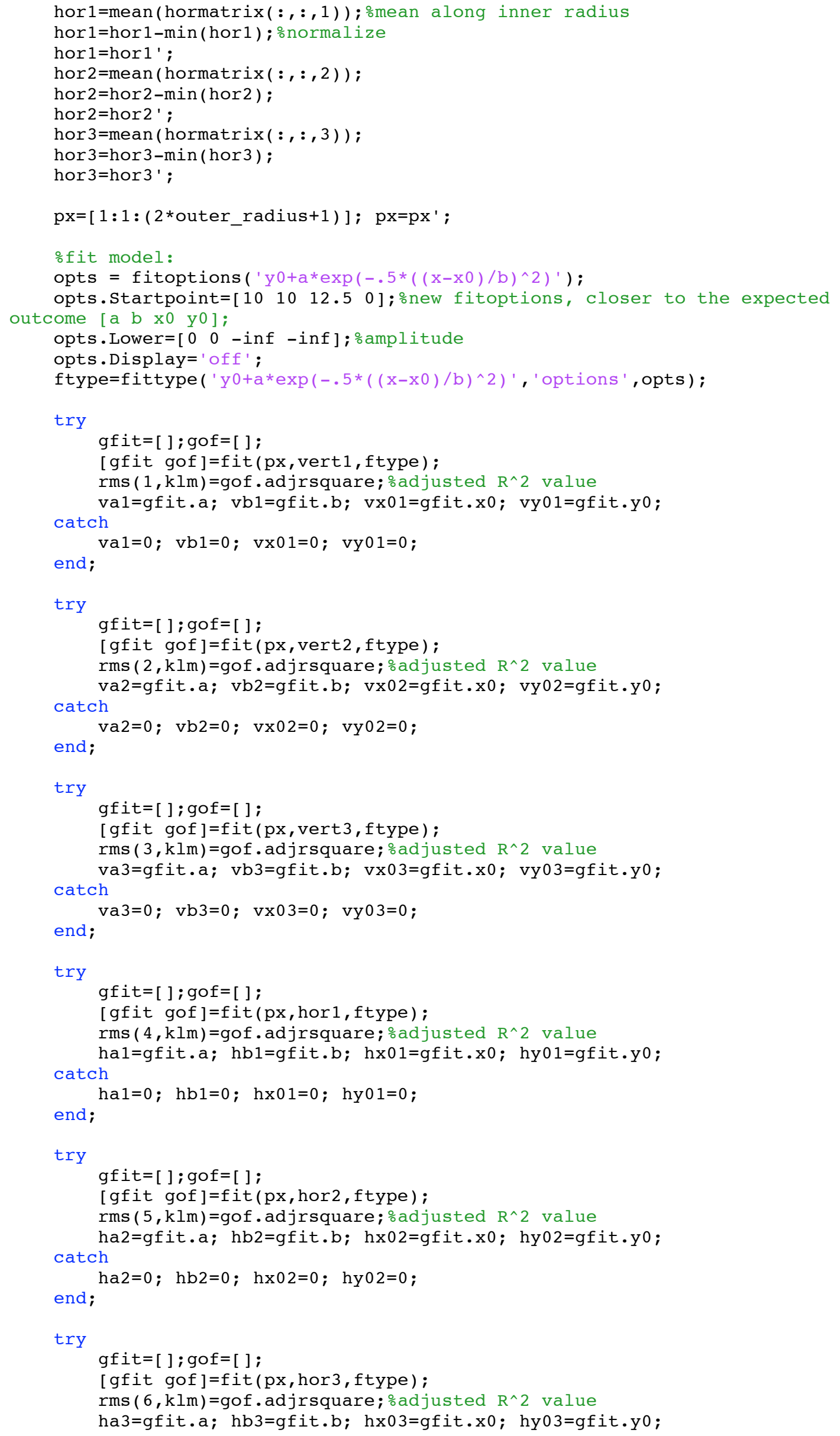


catch ha $3=0 ; h b 3=0 ; h \times 03=0 ; h y 03=0 ;$

end;

hfit $1=[] ; \operatorname{hfit} 2=[] ; \operatorname{hfit} 3=[] ; \operatorname{vfit} 1=[] ; \operatorname{vfit} 2=[] ; \operatorname{vfit} 3=[]$;

hfit $1=\left(\right.$ hy0 $1+$ ha $\left.1 * \exp \left(-.5 *((\mathrm{px}-\mathrm{hx} 01) / \mathrm{hb} 1) \cdot{ }^{\wedge} 2\right)\right)$ ';

hfit $2=\left(\text { hy02 }+ \text { ha } 2 * \exp \left(-.5 *((p x-h x 02) / h b 2) .{ }^{\wedge} 2\right)\right)^{\prime}$ ';

hfit $3=\left(\right.$ hy03+ha $\left.\left.3 * \exp \left(-.5 *((\mathrm{px}-\mathrm{hx} 03) / \mathrm{hb} 3) \cdot{ }^{\wedge} 2\right)\right)\right)^{\prime}$;

vfit $\left.1=\left(\operatorname{vy} 01+\mathrm{va} 1 * \exp \left(-.5 *((\mathrm{px}-\mathrm{vx} 01) / \mathrm{vb} 1) \cdot{ }^{\wedge} 2\right)\right)\right)^{\prime}$;

vfit $\left.2=\left(\operatorname{vy} 02+v a 2 * \exp \left(-.5 *((\mathrm{px}-\mathrm{vx} 02) / \mathrm{vb} 2) \cdot{ }^{\wedge} 2\right)\right)\right)^{\prime}$;

vfit $3=\left(\right.$ vy03+va $\left.\left.3 * \exp \left(-.5 *((\operatorname{px}-\mathrm{vx} 03) / \mathrm{vb} 3) \cdot{ }^{\wedge} 2\right)\right)\right)^{\prime}$;

diffv1=realsqrt ( $\left.\operatorname{sum}\left(\left(\operatorname{vert} 1{ }^{\prime}-\operatorname{vfit} 1\right) \cdot{ }^{\wedge} 2\right)\right)$;

diffv2=realsqrt $\left(\operatorname{sum}\left(\left(\operatorname{vert} 2{ }^{\prime}-\operatorname{vfit} 2\right) \cdot{ }^{\wedge} 2\right)\right)$;

diffv3=realsqrt $\left(\operatorname{sum}\left(\left(\operatorname{vert} 3^{\prime}-\operatorname{vfit} 3\right) \cdot{ }^{\wedge} 2\right)\right)$;

diffh1=realsqrt ( $\operatorname{sum}\left((\right.$ hor 1 '-hfit 1$\left.\left.) \cdot{ }^{\wedge} 2\right)\right)$;

diffh2=realsqrt $\left(\operatorname{sum}\left(\left(\operatorname{hor} 2{ }^{\prime}-\operatorname{hfit} 2\right) \cdot{ }^{\wedge} 2\right)\right)$;

$\operatorname{diffh} 3=$ realsqrt $\left(\operatorname{sum}\left(\left(\operatorname{hor} 3^{\prime}-\operatorname{hfit} 3\right) \cdot{ }^{\wedge} 2\right)\right)$;

ofit matrix coordinates

fitsmatrix $(1, \mathrm{klm})=\mathrm{xx}(\operatorname{xxxxx}(\mathrm{klm}))$; fitsmatrix $(2, \mathrm{klm})=\mathrm{yy}(\operatorname{xxxxx}(\mathrm{klm}))$;

fitsmatrix $(3, \mathrm{klm})=\mathrm{zz}(\operatorname{xxxxx}(\mathrm{klm}))$;

fitsmatrix $(4, \mathrm{klm})=\mathrm{val} ;$ fitsmatrix $(5, \mathrm{klm})=\mathrm{vb} 1 ;$ fitsmatrix $(6, \mathrm{klm})=\mathrm{vx} 01$;

fitsmatrix $(7, \mathrm{klm})=\mathrm{vy} 01 ;$ fitsmatrix $(8, \mathrm{klm})=\operatorname{diffv} 1$;

fitsmatrix $(9, \mathrm{klm})=\mathrm{va} 2$; fitsmatrix $(10, \mathrm{klm})=\mathrm{vb} 2 ; \operatorname{fitsmatrix}(11, \mathrm{klm})=\mathrm{vx} 02$;

fitsmatrix $(12, \mathrm{klm})=\mathrm{vy} 02$; fitsmatrix $(13, \mathrm{klm})=\operatorname{diffv} 2$;

fitsmatrix $(14, \mathrm{klm})=\mathrm{va} 3$; fitsmatrix $(15, \mathrm{klm})=\mathrm{vb} 3 ;$ fitsmatrix $(16, \mathrm{klm})=\mathrm{vx} 03$;

fitsmatrix $(17, \mathrm{klm})=$ vy03; fitsmatrix $(18, \mathrm{klm})=\operatorname{diffv} 3$;

fitsmatrix $(19, \mathrm{klm})=\mathrm{hal} ; \operatorname{fitsmatrix}(20, \mathrm{klm})=\mathrm{hbl} ; \operatorname{fitsmatrix}(21, \mathrm{klm})=\mathrm{hx} 01$; fitsmatrix $(22, \mathrm{klm})=\mathrm{hy} 01$; fitsmatrix $(23, \mathrm{klm})=\operatorname{diffh} 1$;

fitsmatrix $(24, \mathrm{klm})=\mathrm{ha} 2 ; \operatorname{fitsmatrix}(25, \mathrm{klm})=\mathrm{hb} 2 ; \operatorname{fitsmatrix}(26, \mathrm{klm})=\mathrm{hx} 02$;

fitsmatrix $(27, \mathrm{klm})=$ hy02; fitsmatrix $(28, \mathrm{klm})=\operatorname{diffh} 2$;

fitsmatrix $(29, \mathrm{klm})=\mathrm{ha} 3 ; \operatorname{fitsmatrix}(30, \mathrm{klm})=\mathrm{hb} 3 ; \operatorname{fitsmatrix}(31, \mathrm{klm})=\mathrm{hx} 03$;

fitsmatrix $(32, \mathrm{klm})=$ hy03; fitsmatrix $(33, \mathrm{klm})=\operatorname{diffh} 3$;

orealsmatrix coordinates

realsmatrix $(1, \mathrm{klm})=\mathrm{xx}(\operatorname{xxxxx}(\mathrm{klm})) ; \operatorname{realsmatrix}(2, \mathrm{klm})=\mathrm{yy}(\operatorname{xxxxx}(\mathrm{klm}))$;

realsmatrix $(3, \mathrm{klm})=\mathrm{zz}(\operatorname{xxxxx}(\mathrm{klm}))$;

measurer $=2$ *outer radius +1

realsmatrix $(4:$ measurer $+3, \mathrm{klm})=\operatorname{vert} 1$;

realsmatrix (measurer $+4: 2 *$ measurer $+3, \mathrm{klm})=\mathrm{vfit} 1^{\prime}$

realsmatrix $(2 *$ measurer $+4: 3 *$ measurer $+3, \mathrm{klm})=$ vert 2 ;

realsmatrix $(3 *$ measurer $+4: 4 *$ measurer $+3, \mathrm{klm})=$ vfit 2 ';

realsmatrix $(4 *$ measurer $+4: 5 *$ measurer $+3, \mathrm{klm})=$ vert 3 ;

realsmatrix $(5 *$ measurer $+4: 6 *$ measurer $+3, \mathrm{klm})=\mathrm{vfit} 3{ }^{\prime}$;

realsmatrix $(6 *$ measurer $+4: 7 *$ measurer $+3, \mathrm{klm})=$ hor 1 ;

realsmatrix $(7 *$ measurer $+4: 8 *$ measurer $+3, \mathrm{klm})=\mathrm{hfit} 1^{\prime}$;

realsmatrix $(8 *$ measurer $+4: 9 *$ measurer $+3, \mathrm{klm})=$ hor 2 ;

realsmatrix $(9 *$ measurer $+4: 10 *$ measurer $+3, \mathrm{klm})=\mathrm{hfit} 2^{\prime}$;

realsmatrix $(10 *$ measurer $+4: 11 *$ measurer $+3, \mathrm{klm})=$ hor 3 ;

end;

realsmatrix $(11 *$ measurer $+4: 12 *$ measurer $+3, \mathrm{klm})=\mathrm{hfit} 3^{\prime}$;

if ichannel==2

dlmwrite(strcat(savedir, 'tritc ', r1,' fitmatrix.txt'), fitsmatrix); dlmwrite(strcat (savedir,'tritc_',r1, '_realmatrix.txt'), realsmatrix); dlmwrite(strcat(savedir, 'tritc ',r1,' adjrsqrfit.txt'), rms);

elseif ichannel==3

dlmwrite (strcat (savedir,r1,' fitmatrix.txt'), fitsmatrix);

dlmwrite (strcat (savedir, $r 1$, '_realmatrix.txt'), realsmatrix);

dlmwrite (strcat (savedir,r1,' adjrsqrfit.txt'), rms) ;

elseif ichannel==1

dlmwrite (strcat (savedir,rl,' fitmatrix gr.txt'), fitsmatrix);

dlmwrite (strcat (savedir,rl, '_realmatrix_gr.txt'), realsmatrix);

end

dlmwrite(strcat (savedir,r1,' adjrsqrfit gr.txt'), rms) 


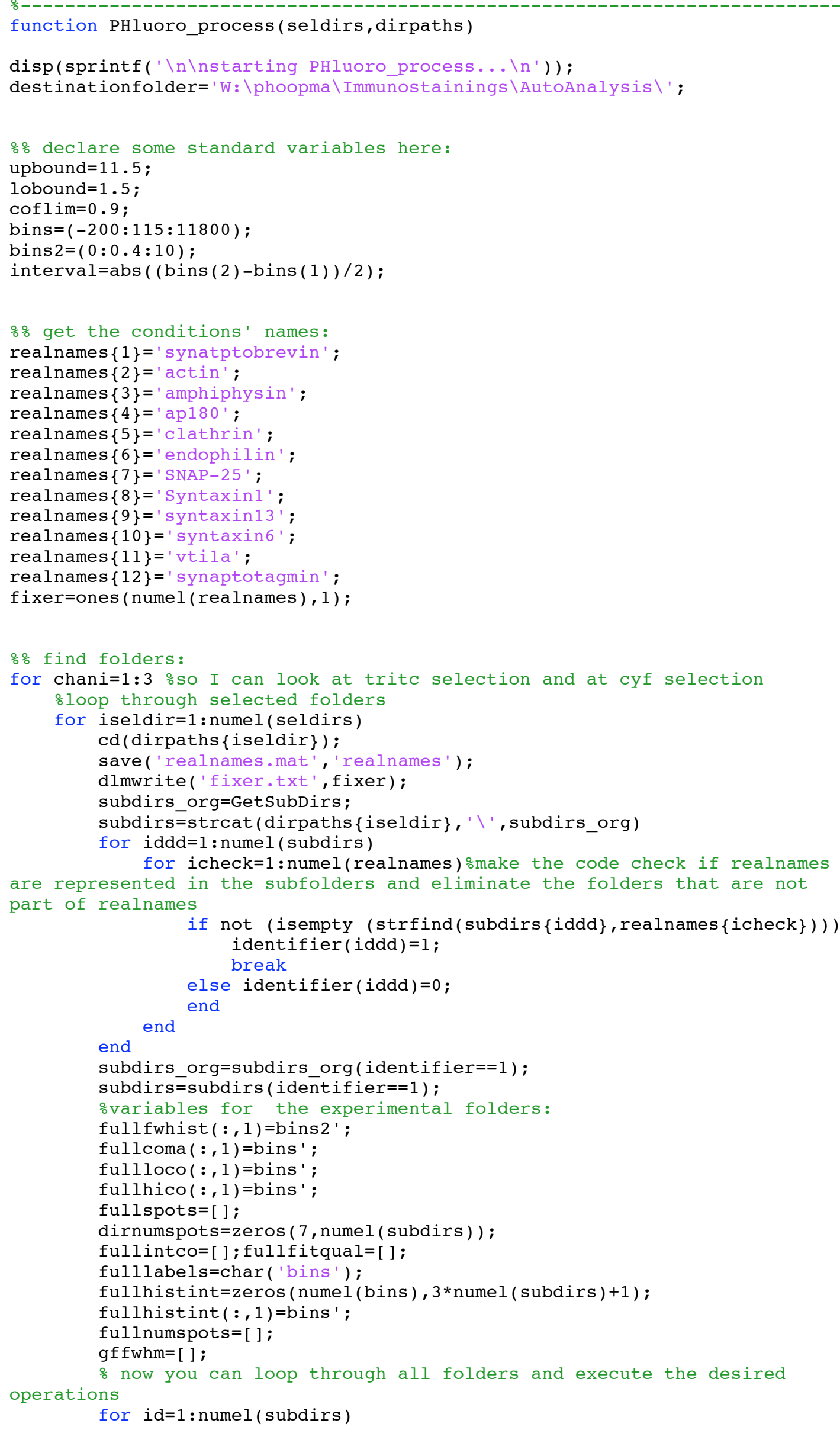




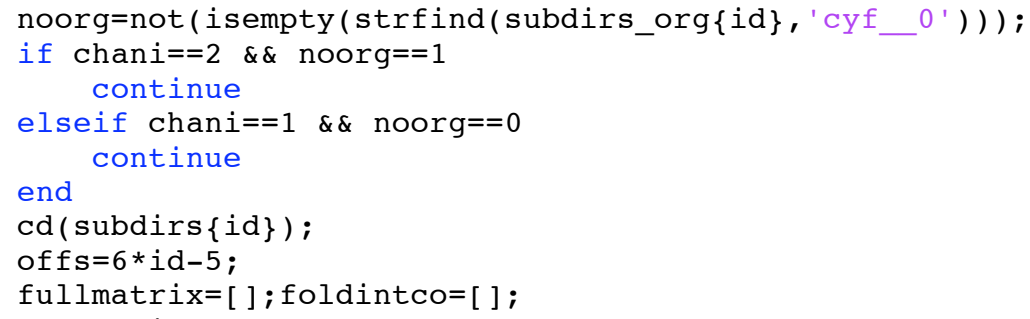


foldintco_id=vertcat (foldintco, foldimage) ;

dlmwrite('tritc_all_intens_coeff.txt',foldintco_id);

dlmwrite('tritc all mean ccoeff.txt', vertcat(foldmcoeff,foldimage)); case 3 dlmwrite('tritc_all_fitqual.txt',foldfitqual);

dlmwrite( 'all_fwhm.txt', fwhm') ;

foldintco_id=vertcat (foldintco, foldimage) ;

dlmwrite('all_intens_coeff.txt',foldintco_id);

dlmwrite( 'all_mean_ccoeff.txt', vertcat(foldmcoeff, foldimage)) ;

case 1 dlmwrite('all fitqual.txt', foldfitqual);

dlmwrite('all fwhm gr.txt',fwhm');

foldintco_id=vertcat (foldintco, foldimage) ;

dlmwrite('all_intens_coeff_gr.txt',foldintco_id);

dlmwrite('all mean ccoeff gr.txt', vertcat(foldmcoeff,foldimage));

end

dlmwrite('all_fitqual_gr.txt',foldfitqual);

switch chani

case 2

save('tritc catmatrices.mat', 'fullmatrix', 'deviats', 'fwhm', 'foldintco id', 'f oldfitqual' );

case 3

save('catmatrices.mat', 'fullmatrix', 'deviats', 'fwhm', 'foldintco id', 'foldfit qual') ;

case 1

save('catmatrices_gr.mat', 'foldintco_id'); \%add the other

ones here!

end

\% make histogram

greenint =foldintco $(4,:)$;

tritcint $=$ foldintco $(5,:)$;

cyfint=foldintco $(6,:)$;

numspot=numel (greenint);

dirnumspots $(1$, id $)=$ numspot ;

g1=hist (greenint, bins) *100/numspot;

t $1=$ hist (tritcint, bins) $* 100 /$ numspot;

c1=hist (cyfint, bins ) *100/numspot;

offset $=i d * 3-1$;

fullhistint $(:$, offset $)=g 1$ ';

fullhistint $(:$, offset +1$)=t 1^{\prime}$;

fullhistint ( : , offset +2$)=\mathrm{c} 1^{\prime}$;

fulllabels=char $($ fulllabels, $\operatorname{char}(\operatorname{subdirs}$ org $($ id $)))$;

$\%$ getfindloop

cgt $=$ foldintco $(7,:)$;

$\operatorname{cgc}=$ foldintco $(8,:)$;

ctc $=$ foldintco $(9,:)$;

cmgt $=$ foldintco $(10,:)$;

cmgc $=$ foldintco $(11,:)$;

$\operatorname{cmtc}=$ foldintco $(12,:)$;

$\operatorname{mgt} 1=[] ; \operatorname{mgt} 2=[] ; \operatorname{mgc} 1=[] ; \operatorname{mgc} 2=[] ; \operatorname{mtc} 1=[] ; \operatorname{mtc} 2=[] ;$

for $i{ }^{\prime}=1$ : numel (bins)

bins (iic) +interval);

eg=find (greenint>bins (iic)-interval \& greenint < $<$

bins (iic) +interval);

et $=$ find (tritcint $>$ bins (iic)-interval \& tritcint $<=$

bins (iic) +interval);

ec $=$ find (cyfint>bins (iic)-interval \& cyfint $<=$

if numel (eg) $>0$

mgt $1($ i ic $)=\operatorname{median}(\operatorname{cgt}($ eg $))$;

else

$\operatorname{mgc} 1($ i ic $)=\operatorname{median}(\mathrm{cgc}(\mathrm{eg}))$; 


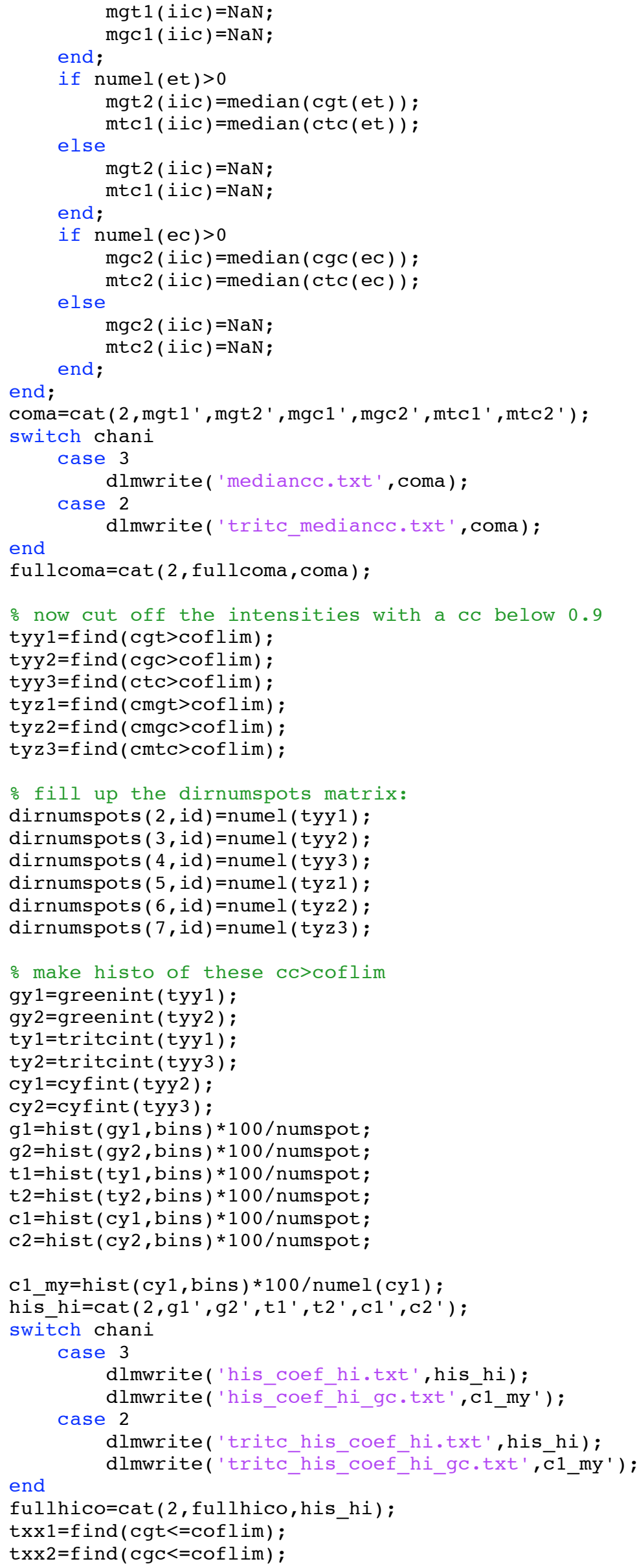


txx3=find $(\mathrm{ctc}<=\operatorname{cof} \mathrm{lim})$;

gx1=greenint $(\operatorname{txx} 1)$;

gx2=greenint $(t \times x 2)$;

tx $1=$ tritcint $(\operatorname{txx} 1)$;

tx2=tritcint $(\operatorname{txx} 3)$;

$\operatorname{cx} 1=\operatorname{cyfint}(\operatorname{txx} 2)$;

$\operatorname{cx} 2=\operatorname{cyfint}(\operatorname{txx} 3)$;

gs $1=$ hist $($ gx 1 ,bins $) * 100 /$ numspot; $\%$ if you want it scaled to $100 \%$

then divide by numgr $2 ;$ /numgr scales to total spots

gs $2=$ hist $(\operatorname{gx} 2$, bins $) * 100 /$ numspot;

ts $1=$ hist $(\operatorname{tx} 1$, bins $) * 100 /$ numspot;

ts $2=$ hist $($ tx 2 , bins $) * 100 /$ numspot;

$\operatorname{cs} 1=$ hist $(\operatorname{cx} 1$, bins $) * 100 /$ numspot;

cs $2=$ hist $(\mathrm{cx} 2$, bins $) * 100 /$ numspot;

his_lo=cat $\left(2, g s 1^{\prime}, g s 2^{\prime}, \operatorname{ts} 1^{\prime}, \operatorname{ts} 2^{\prime}, \operatorname{cs} 1^{\prime}, \operatorname{cs} 2^{\prime}\right)$;

switch chani

case 3

case 2

dlmwrite('his coef_locc.txt', his_lo);

end

dlmwrite('tritc_his_coef_locc.txt',his_lo);

fullloco=cat $(2$, fullloco,his_lo);

\% getelementsofmatrix

if chani =1

for $\mathrm{nnn}=1: 6$

$\mathrm{fw}\{\mathrm{nnn}\}=\mathrm{fwhm}(\mathrm{nnn},:)$;

$\mathrm{h}\{\mathrm{nnn}\}=\mathrm{hist}(\mathrm{fw}\{\mathrm{nnn}\}$, bins 2$) * 100 /$ numspot;

end

$\mathrm{hi}=\operatorname{cat}(1, \mathrm{~h}\{1\}, \mathrm{h}\{2\}, \mathrm{h}\{3\}, \mathrm{h}\{4\}, \mathrm{h}\{5\}, \mathrm{h}\{6\})$;

switch chani

case 3

dlmwrite('fw_hist.txt',hi');

dlmwrite ('fw_hist_bins.txt' ,bins2');

case 2

dlmwrite('tritc fw hist.txt',hi');

end

dlmwrite('tritc_fw_hist_bins.txt',bins2');

fullfwhist ( : , offs+1:1:offs+6)=hi ' ;

fullnumspots=cat $(2$, fullnumspots, dirnumspots ) ;

fullspots $=$ cat $(2$, fullspots, fwhm );

end

gffwhm=cat $(2, g f f w h m, f w h m)$;

\% resume parent

fullintco $=\operatorname{cat}(2$, fullintco, foldintco $)$;

if $\sim \operatorname{rem}(\mathrm{id}, 2)$

end

end

cd ... ;

if chani $=1$

elements=numel (fullspots $(1,:))$;

gf $1=h i s t(f u l l s p o t s(1,:)$, bins 2$)$;

tf $1=$ hist $(f u l l s p o t s(2,:)$, bins 2$)$;

cf $1=$ hist $($ fullspots $(3,:)$, bins 2$)$;

fwhisto $(:, 1)=$ bins 2 ;

fwhisto $(:, 2)=g f 1 * 100 /$ elements ;

fwhisto $(:, 3)=t f 1 * 100 /$ elements ;

fwhisto $(:, 4)=\operatorname{cf} 1 * 100 /$ elements ;

g2=hist (fullspots $(4,:)$, bins 2$)$;

t2 $=$ hist (fullspots $(5,:)$, bins2);

c2 $=$ hist (fullspots $(6,:)$, bins 2$)$;

fwhisto $(:, 5)=g 2 * 100 /$ elements :

fwhisto $(:, 6)=t 2 * 100 /$ elements ;

end

fwhisto $(:, 7)=\mathrm{c} 2 * 100 /$ elements ;

end

subbers $\{$ iseldir $\}=$ subdirs org ; 
end

if nargin $\sim=2$

disp(sprintf('\n...done concatenating all files.\t(\%2.1f

minutes ) $\backslash \mathrm{n}^{\prime}$, toc/60));

else

end

disp(sprintf('\n...done concatenating all files.'));

$\%$ call more analysis programs:

[cutoff

coflim]=pH_gp_catmonopoly_GetThresh(seldirs, destinationfolder, subbers, realna mes, lobound, upbound ) ;

dirgenpool=pH_findtritccyf_genpool(seldirs, destinationfolder, subbers, cutoff , realnames, lobound, upbound, coflim);

dirtritc $=\mathrm{pH}$ findtritccyf_tritcclick(seldirs, destinationfolder, subbers, cutoff , realnames, Iobound, upbound, coflim);

dirgreen=pH_findtritccyf_green(seldirs, destinationfolder, subbers, cutoff, real names, lobound, upbound, coflim).

pH_finalresult (dirgenpool, dirtritc, dirgreen, seldirs) ;

pH_catexpi_intco(seldirs, destinationfolder, subbers, cutoff, realnames, lobound, upbound, $\operatorname{cof} \bar{l} \mathrm{im})$;

pH catexpi intco tritcclick(seldirs, destinationfolder, subbers, cutoff, realnam es, lobound, upbound, coflim);

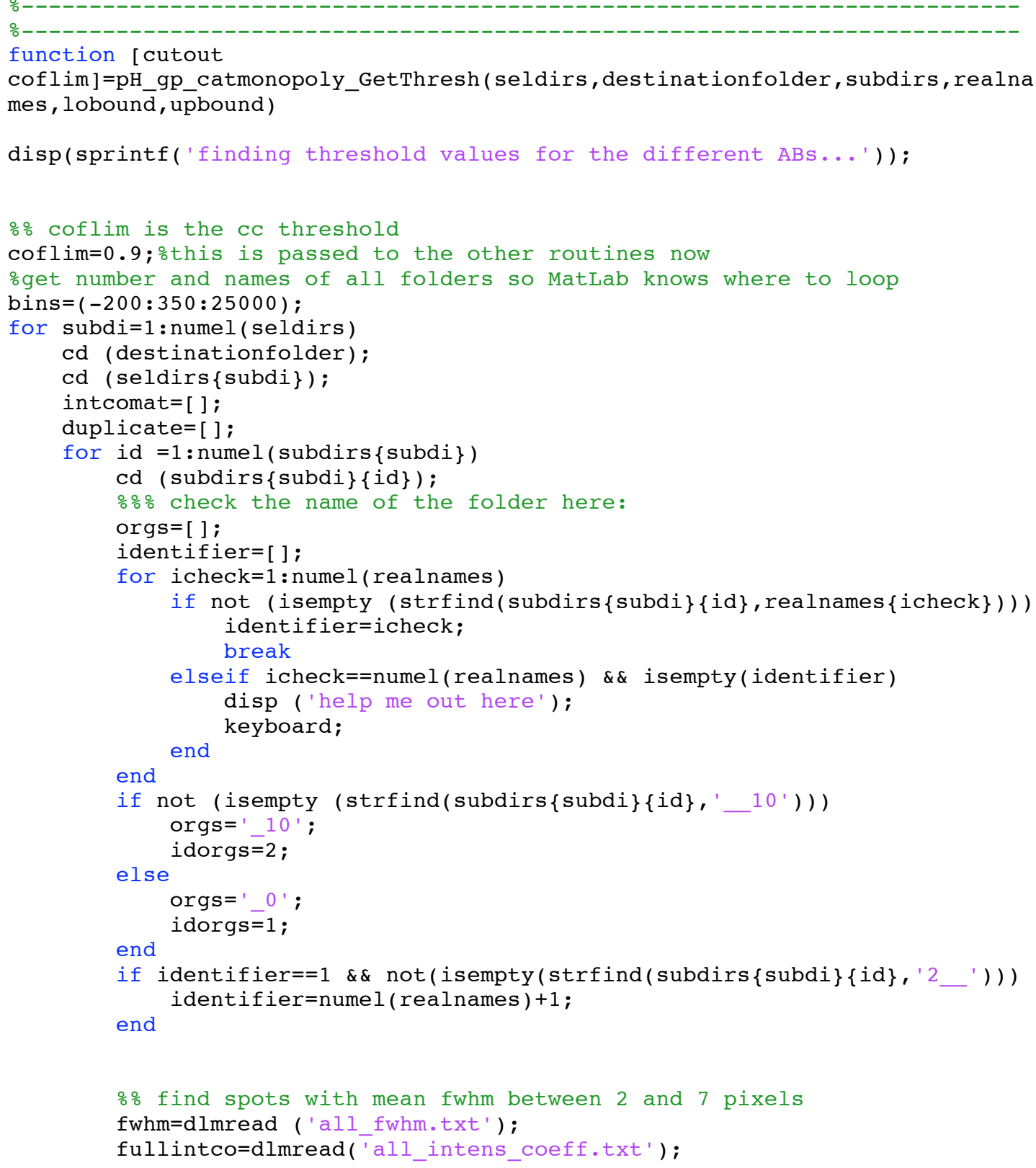




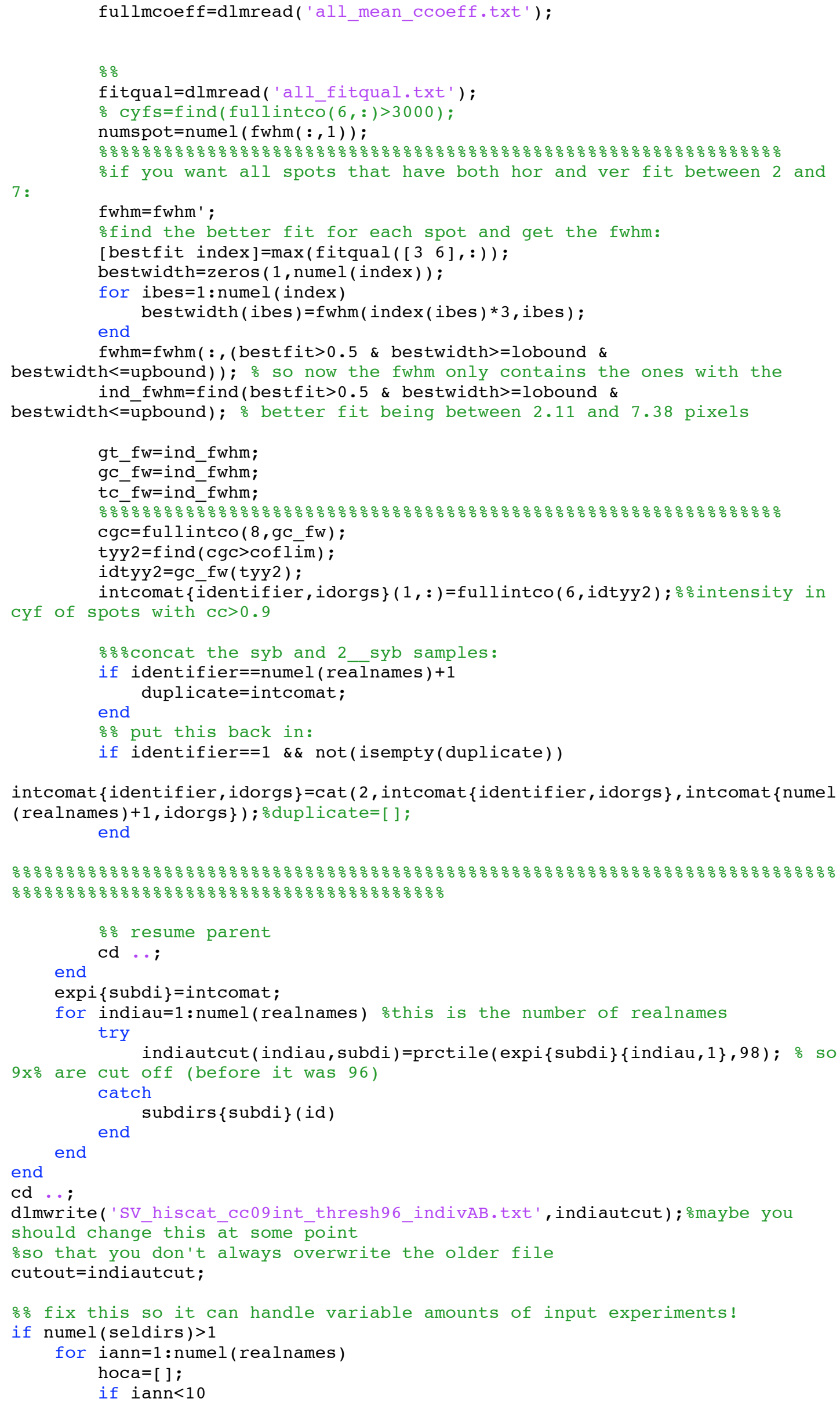


else continue

\% $\%$ find spots with mean fwhm between 2 and 7 pixels

fwhm=dlmread ('all_fwhm.txt') ;

fullintco=dlmread ('all intens coeff.txt') ;

fullmcoeff=dlmread ( 'al $\bar{l}$ mean_ccoeff.txt' ) ;

fitqual=dlmread ('all_fitqual.txt' ) ;

if identifier $==1 \& \& \operatorname{not}($ isempty (dup))

fwhm $=\operatorname{cat}(1, \mathrm{fwhm}, \operatorname{dup}\{1$, idorgs $\}) ;$

fullintco $=\operatorname{cat}(2$, fullintco, $\operatorname{dup}\{2$, idorgs $\})$;

fitqual=cat $(2$, fitqual, $\operatorname{dup}\{3$, idorgs $\})$;

end

fullmcoeff=cat $(2, f u l l m c o e f f, \operatorname{dup}\{4$, idorgs $\})$;

the

\% activate this cell to use the mean $\mathrm{Cc}$ instead of the max of

numspot $=$ numel $(\mathrm{fwhm}(:, 1))$;

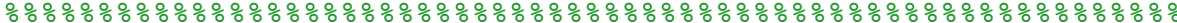

and 7 :

oif you want all spots that have both hor and ver fit between 2

$\mathrm{fwhm}=\mathrm{fwhm}^{\prime}$;

ofind the better fit for each spot and get the fwhm:

[ bestfit index] =max (fitqual $\left.\left(\left[\begin{array}{ll}3 & 6\end{array}\right],:\right)\right)$;

bestwidth=zeros $(1$, numel (index) );

for ibes $=1$ : numel (index)

bestwidth $($ ibes $)=f w h m($ index $($ ibes $) * 3$, ibes $)$;

end

fwhm=fwhm(:, (bestfit>0.5\& bestwidth>=lobound \&

bestwidth<=upbound)); \% so now the fwhm only contains the ones with the ind fwhm=find(bestfit>0.5\& bestwidth>=lobound \&

bestwidth<=upbound );

gt_fw=ind_fwhm

gc_fw=ind_fwhm;

tc $\mathrm{fw}=\mathrm{ind}$ fwhm;

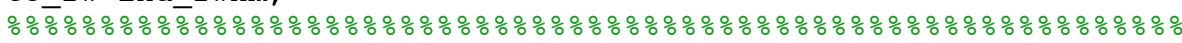

cgt =fullintco ( 7 , gt_fw );

$\mathrm{cgc}=\mathrm{fullintco}\left(8, \mathrm{gc}^{-} \mathrm{fw}\right)$;

ctc $=f u l l i n t c o(9$, tc_fw $)$.

cmgt $=f u l l i n t c o\left(10, g t \_f w\right)$;

$\mathrm{cmgc}=\mathrm{fullintco}\left(11, \mathrm{gc} \_\mathrm{fw}\right)$;

cmtc $=f u l l i n t c o(12$, tc_fw $)$;

$\%$ now cut off the intensities with a cc below 0.9 and make a

histogram

tyy $1=f$ ind $(\operatorname{cgt}>\operatorname{cof} l i m)$;

$i \operatorname{dtyy} 1=g t \_f w(t y y 1)$;

tyy $2=f$ ind $(\mathrm{cgc}>\operatorname{coflim})$;

idtyy $2=g c \_f w(t y y 2)$;

tyy $3=f$ ind $(c t c>\operatorname{cof} l i m)$;

idtyy $3=t c \_f w($ tyy 3$)$;

tyz $1=$ find $(\mathrm{cmgt}>\operatorname{cof} \lim )$;

idtyz $1=g t \quad f w(t y z 1)$;

tyz2 $=$ find $(\mathrm{cmgc}>\operatorname{coflim})$;

idtyz $2=g c$ fw $($ tyz 2$)$;

tyz $3=$ find $(\mathrm{cmtc}>\operatorname{coflim})$;

idtyz3=tc_fw $($ tyz3);

$\operatorname{cutc}=f i n d(f u l l i n t c o(6,:)>\operatorname{cutoff}($ identifier, subdi $))$;

idtyy $1=$ intersect ( idtyy 1, cutc $)$;

idtyy $2=$ intersect ( idtyy 2 , cutc) ;

idtyy $3=$ intersect ( idtyy3, cutc) ; 


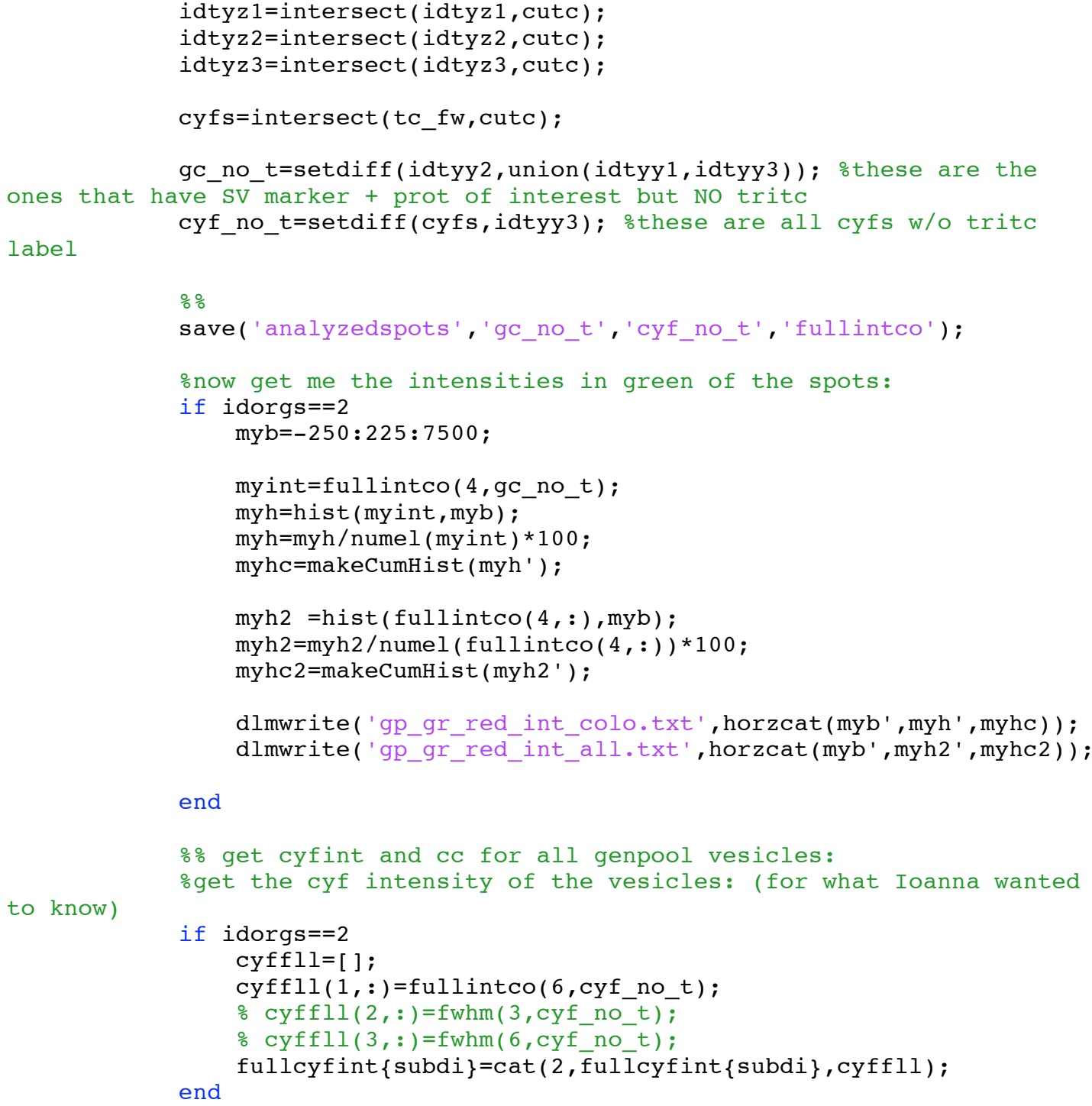

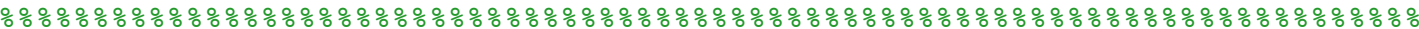

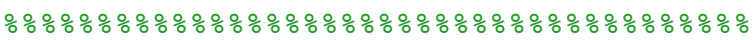

dirnumspots $(1$, identifier, idorgs $)=$ numspot;

dirnumspots $(2$, identifier, idorgs $)=$ numel (idtyy 1$)$;

dirnumspots $(3$, identifier, idorgs $)=\operatorname{numel}($ idtyy 2$)$;

dirnumspots $(4$, identifier, idorgs $)=$ numel (idtyy 3$)$;

dirnumspots $(5$, identifier, idorgs $)=$ numel (idtyz 1$)$;

dirnumspots $(6$, identifier, idorgs $)=\operatorname{numel}($ idtyz 2$)$;

dirnumspots $(7$, identifier, idorgs $)=\operatorname{numel}($ idtyz 3$)$;

dirnumspots ( 8 , identifier, idorgs) $=$ numel (gc no $t$ ); onumber of

spots that have SV marker + prot of interest but NO tritc

$\operatorname{dirnumspots}(9$, identifier, idorgs $)=\operatorname{numel}($ cyf_no_t $)$; $\%$ total number

of vesicles w/o tritc

tritcspots $(1$, identifier, idorgs $)=\operatorname{numel}($ cyfs $)$;

tritcspots ( 2 , identifier, idorgs $)=$ numel (idtyy 3$)$;

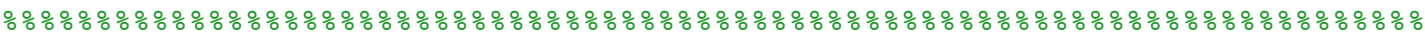
응 응 $\% \% \%$

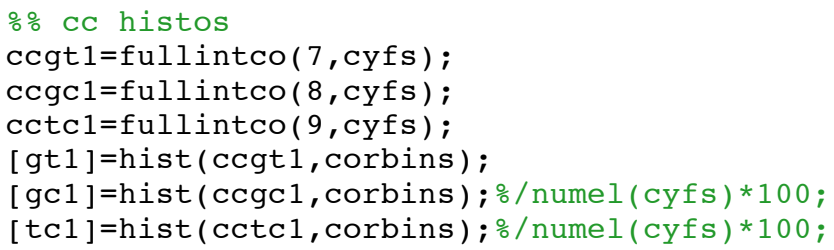




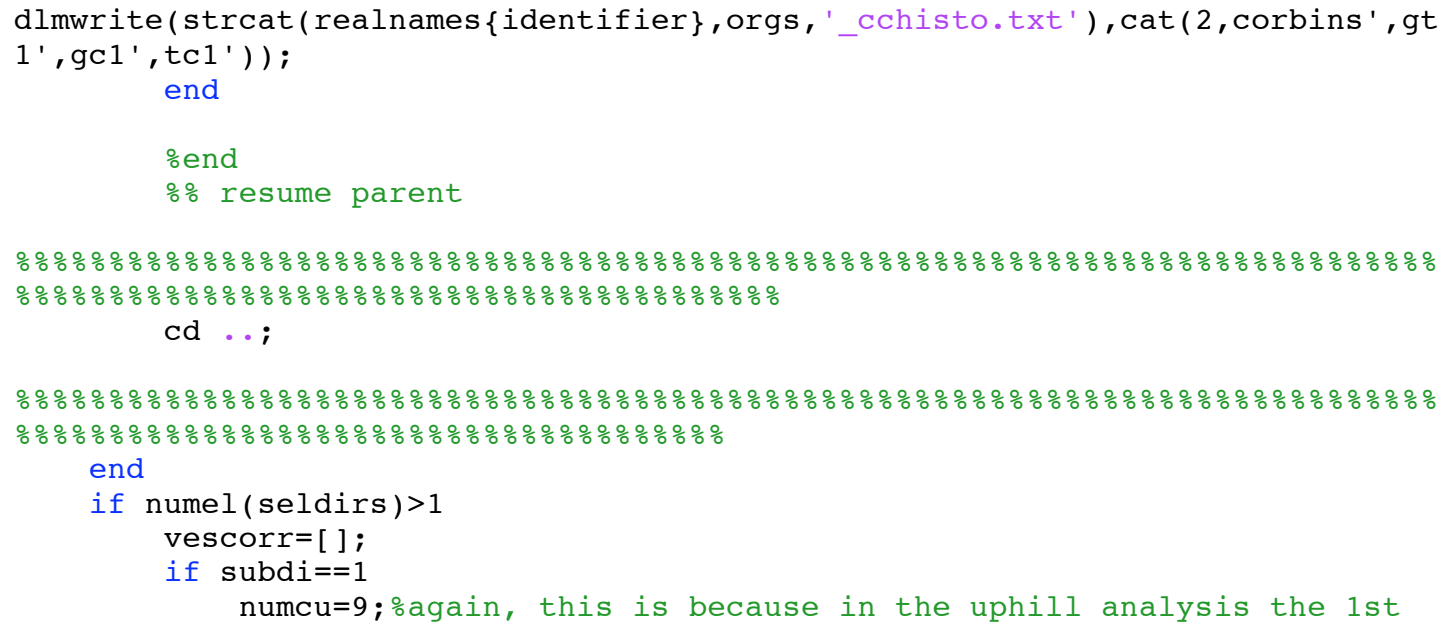

dlmwrite( 'numspots_autocut_0_10.txt' , (cat(2,dirnumspots ( : , : , 1$)$, dirnumspots (: ,$: 2$ )) )' ) ;

dlmwrite('tritcspots_autocut_0_10.txt', (cat(2, tritcspots(:,:,1), tritcspots (: ,$: 2$ )) ) ') ; end dlmwrite('numspots_autocut_spotorder.txt', realnames ); 

end

aunum $($ ite, 1$)=$ mean $($ hoca 3,2$)$;

aunum ( ite, 3 ) =mean ( hoca 4,2$)$; end

aunum $($ ite, 2$)=\operatorname{std}($ hoca 3,0$) / \operatorname{sqrt}($ numel $($ idiot $))$;

aunum $($ ite, 4$)=\operatorname{std}($ hoca 4,0$) / \operatorname{sqrt}($ numel $($ idiot $))$;

dlmwrite(strcat('immo_autocut_mean_of_',num2str(numel(seldirs)), '_genpool.tx $t^{\prime}$ ), aunum);

end

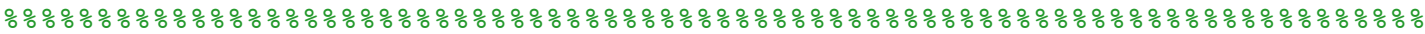
응 $\% \circ \%$

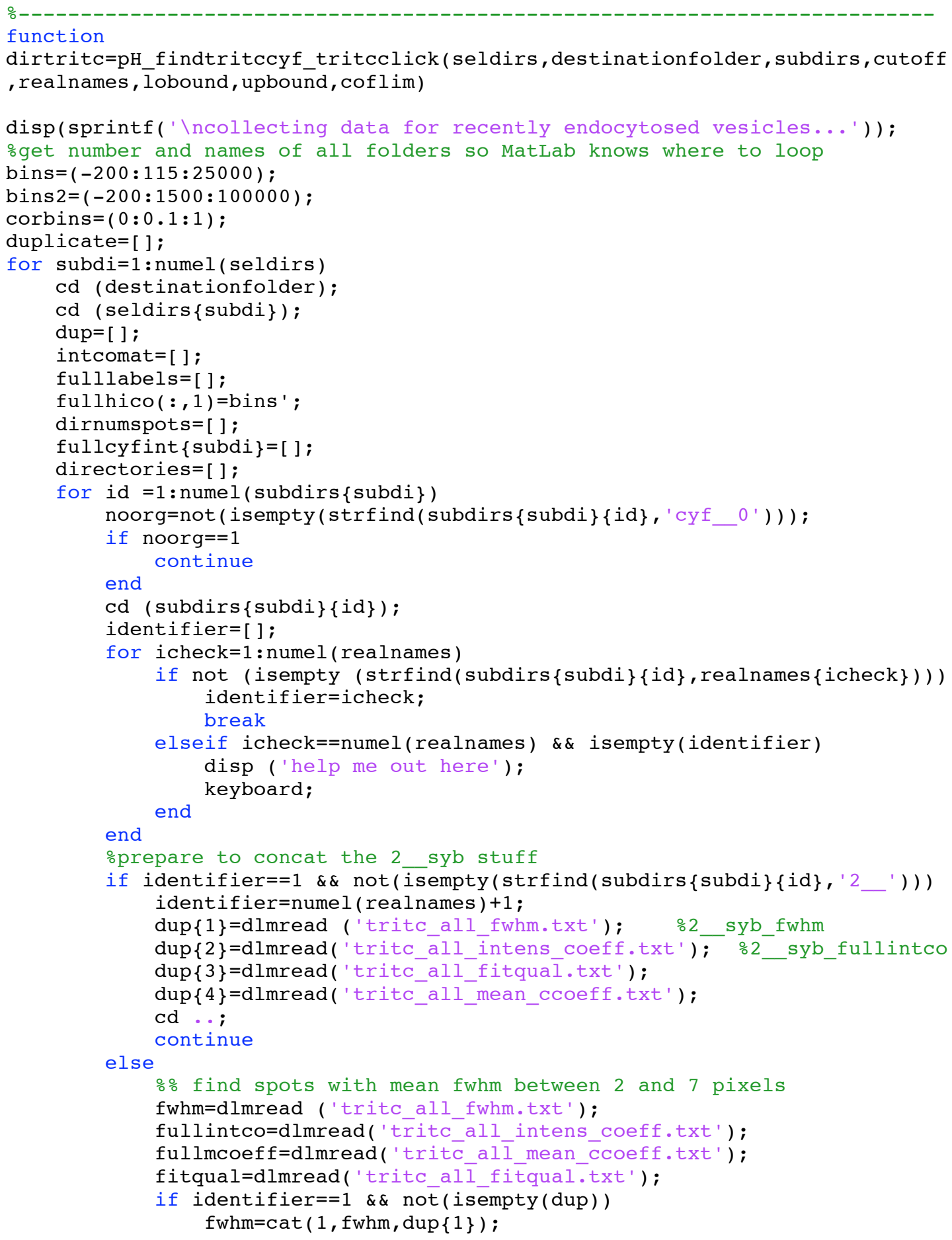




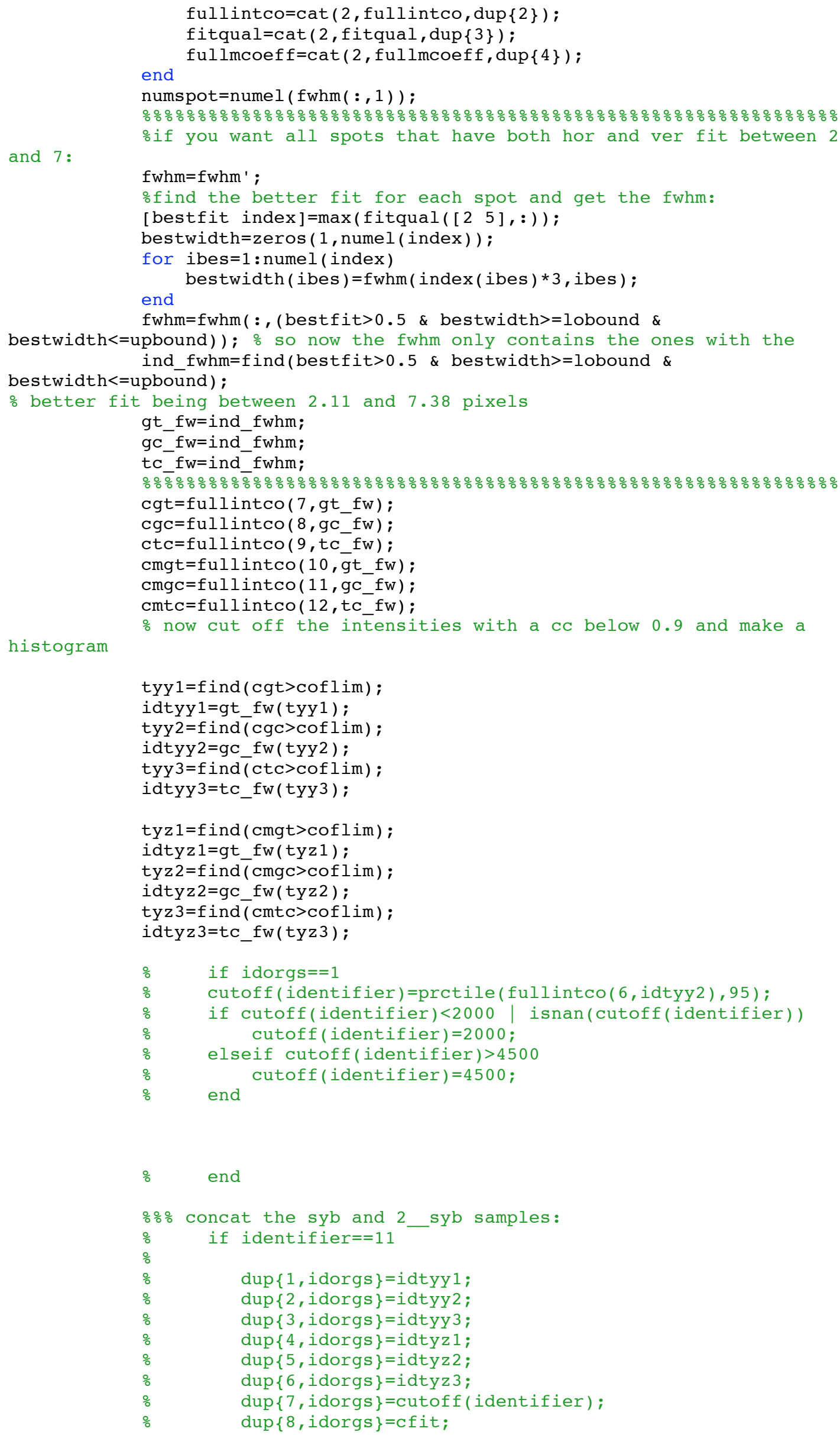




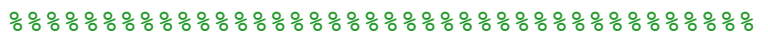

dirnumspots $(1$, identifier $)=$ numspot ;

dirnumspots $(2$, identifier $)=\operatorname{numel}($ idtyy 1$)$;

dirnumspots $(3$, identifier $)=\operatorname{numel}($ idtyy 2$)$;

dirnumspots $(4$, identifier $)=\operatorname{numel}($ idtyy 3$)$;

dirnumspots $(5$, identifier $)=\operatorname{numel}($ idtyz 1$)$;

dirnumspots $(6$, identifier $)=\operatorname{numel}($ idtyz 2$)$;

dirnumspots $(7$, identifier $)=\operatorname{numel}($ idtyz 3$)$;

dirnumspots $(8$, identifier $)=$ numel (id_gtc); onumber of spots that

have SV marker + prot of interest and tritc

dirnumspots $(9$, identifier $)=\operatorname{numel}($ idtyy 3$)$; ototal number of

vesicles

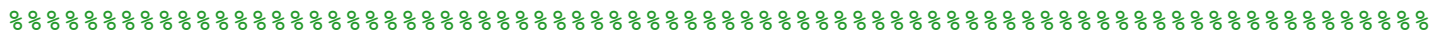

응 응 $\%$

\% CC histos:

ccgt $1=f u l l i n t c o(7, \operatorname{idtyy} 3)$;

$\operatorname{ccgc} 1=f u l l i n t c o(8, \operatorname{idtyy} 3)$;

cctc $1=$ fullintco $(9, \operatorname{idtyy} 3)$;

[ gt 1 ] =hist $(\operatorname{ccgt} 1$, corbins $) ; \% /$ numel ( cyfs) $* 100$;

$[$ gcl $]=h i s t(\operatorname{ccgc} 1$, corbins $) ; \% / \operatorname{numel}($ cyfs $) * 100$;

$[$ tcl $1=\operatorname{hist}(\operatorname{cctc} 1, \operatorname{corbins}) ; \% /$ numel (cyfs) $* 100$;

dlmwrite (strcat ('tritc_', realnames $\{$ identifier\}, '_10 ', '_cchisto.txt'),cat(2,c orbins', gt $1^{\prime}, g c 1$ ', tc $\left.1{ }^{\prime}\right)$ ) ;

end

\% resume parent

$\mathrm{cd} \ldots$;

end

if numel(seldirs) $>1$

vescorr $=[$ ] ;

if $\operatorname{subd} i==1$

numcu=9; \%again, this is because in the uphill analysis the 1st

one didn't have realnames(10)

else

end

numcu=10;

for ivesc $=1$ : numcu

vescorr $($ ivesc, 1$)=\operatorname{dirnumspots}(8$, ivesc $) ;$

dirnumspots ( 8 , ivesc, 1 )*dirnumspots (1,ivesc, 2)/dirnumspots ( 1 , ivesc, 1 ); \%this

takes into consideration on how many spots I clicked

vescorr $($ ivesc, 2$)=\operatorname{dirnumspots}(9$, ivesc $) ; \%-$

dirnumspots ( 9 , ivesc, 1$)$ *dirnumspots (1, ivesc, 2$) / \operatorname{dirnumspots~}(1$, ivesc, 1$)$; \%this

takes into consideration on how many spots I clicked

vescorr $($ ivesc , 3$)=\operatorname{vescorr}($ ivesc, 1$) / \operatorname{vescorr}($ ivesc , 2$) * 100$;

end

vescorr $($ ivesc, 4$)=\operatorname{vescorr}(i \operatorname{vesc}, 3) / \operatorname{vescorr}(1,3) * 100$;

end

if not (isempty (dirnumspots))

dirtritc $=$ dirnumspots;

dlmwrite('tritc numspots autocut $010 . t x t$ ',dirnumspots'); end

dlmwrite('tritc_numspots_autocut_spotorder.txt', realnames) ;

cd ...;

if numel (seldirs) $>1$ end

exi $\{$ subdi $\}=$ vescorr;

end

\%응 vesicle brightness histos

for $\operatorname{vbi=1}$ : numel (seldirs)

end $\mathrm{vb}(:, \mathrm{vbi})=$ hist $($ fullcyfint $\{\mathrm{vbi}\}, \mathrm{bins} 2) / \operatorname{numel}($ fullcyfint $\{\mathrm{vbi}\}) * 100$;

mvb=mean $(v b, 2)$;

stdvb=std $(v b, 0,2) / r e a l s q r t($ numel ( seldirs ) ) ;

dlmwrite('vesicle_brightness_tritcclick.txt', cat (2,bins2',mvb, stdvb)) ;

\% change this so it handles variable numbers of experiments:

if numel (seldirs) $>1$

for ite $=1$ : numel (realnames)

hoca $3=[$ ] hoca $4=[$ ] ;

if ite<numel (realnames) 


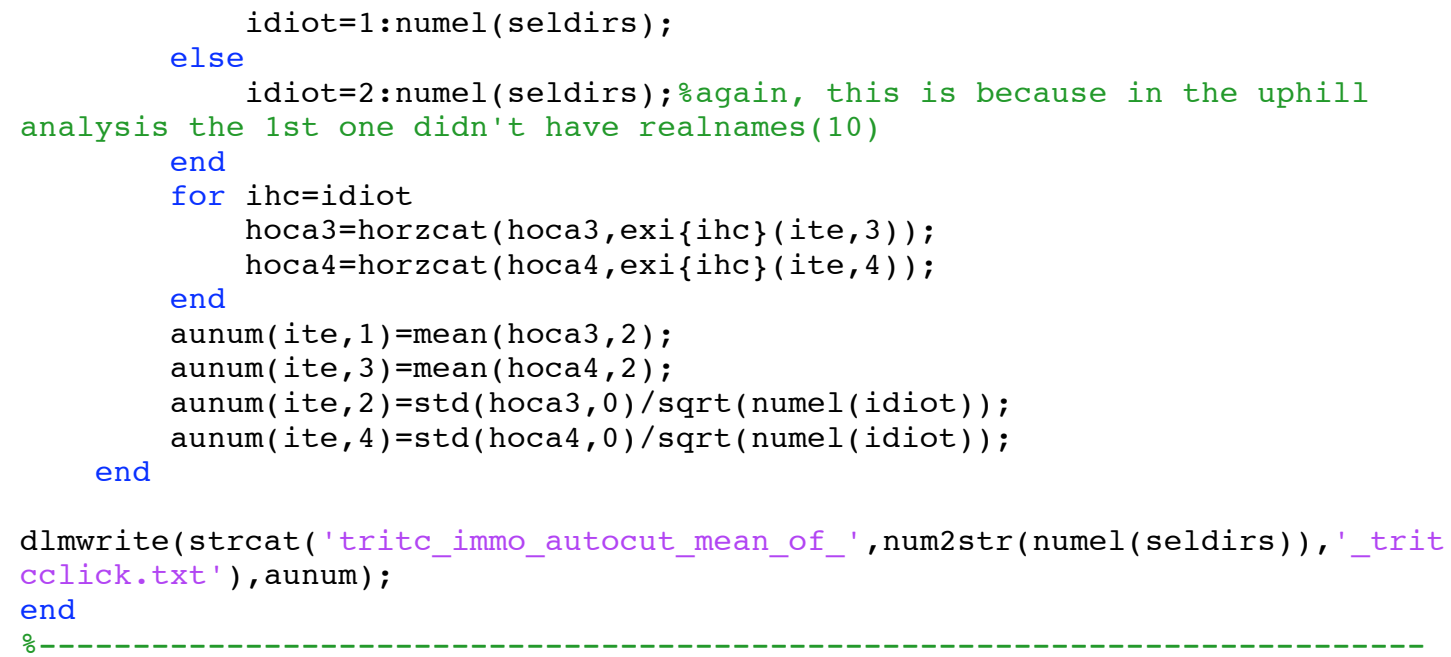

dlmwrite(strcat('tritc immo autocut mean of ', num2str(numel(seldirs)), ' trit cclick.txt'), aunum); 


\section{CURRICULUM VITAE}

Contact Details

Peer Hoopmann

ENI Göttingen

Grisebachstr. 5

Phone: 0551 - 3913955

37077 Göttingen

E-mail: phoopma@gwdg.de

\section{EDUCATION}

since 10/2007:

International Max-Planck Research School (IMPRS), Göttingen $\mathrm{PhD}$ in the MSc/PhD program „Molecular Biology“

Thesis work at the European Neuroscience Institute Göttingen, STED Microscopy of Synaptic Function (Dr. Silvio Rizzoli)

10/2006 - 05/2008: International Max-Planck Research School (IMPRS), Göttingen MSc in the MSc/PhD program „Molecular Biology” Thesis work at the European Neuroscience Institute Göttingen, STED Microscopy of Synaptic Function (Dr. Silvio Rizzoli)

Title: „Fluorescence microscopy investigation of isolated synaptic vesicles"

04/2003 - 09/2006: Johannes-Gutenberg-University Mainz

Biology

,Vordiplom' awarded in October 2005

06/2002: Domgymnasium Verden

High school degree (,Abitur')

08/1998 - 05/1999: Cary High School, NC, USA

High School Year

\section{RESEARCH PROJECTS AND WORKSHOPS}

03/2010 - 03/2010: atrain \& Roche Diagnostics, Mannheim

3-day Leadership-Workshop: Acquiring leadership principles through case studies and role-plays within a framework of logistics optimization

09/2009 - 09/2009: Institut für Management-Entwicklung \& Roche Diagnostics, Penzberg

3-day Corporate-Strategic-Planning-Simulation TOPSIM II - General Management: Acquiring business concepts as executive board of the fictitious „Coolklima $\mathrm{AG}^{\prime \prime}$

12/2008 - 12/2008: The Boston Consulting Group, Munich

2-day Business-Consulting-Workshop for Natural Scientists: Developing an NGO-strategy to fight malaria

04/2007 - 06/2007: Sartorius AG, Göttingen

Research project in the Biotechnology Department (Dr. Karl Pflanz / Dr. Uwe Andag)

Topic: Establishing a lateral flow assay for the detection of THC

02/2007 - 04/2007: Max-Planck Institute for Biophysical Chemistry, Göttingen Research project in the Neurobiology Department (Prof. Reinhard Jahn) Topic: The role of $\mathrm{pH}$ and $\mathrm{Ca}^{2+}$ on endosome fusion 
01/2007 - 02/2007: Max-Planck Institute for Biophysical Chemistry, Göttingen Research project in the Chromatin Biochemistry Laboratory (Dr. Wolfgang Fischle)

Topic: Interaction of histone $\mathrm{H} 3 \mathrm{~K} 9 \mathrm{me}^{3}$ and HP1

06/2006 - 07/2006: Johannes-Gutenberg-University Mainz

Research project at the 'Institut für Allgemeine Botanik' (Prof. Harald Paulsen)

Topic: Site-directed mutagenesis and kinetic analysis of the xantophyll cycle enzyme violaxanthin-deepoxidase

\section{AWARDS AND STIPENDS}

since 01/2009:

09/2008:

since 08/2008:

09/2006 - 08/2007: e-fellows.net Stipend

$2^{\text {nd }}$ Poster-Prize

5th International PhD Student Symposium Horizons in Molecular Biology, Göttingen

Boehringer Ingelheim Fonds PhD Fellowship

International Max-Planck Research School Stipend

\section{TEACHING EXPERIENCE}

06/2010 - 06/2010: Teaching of Practical Advanced Methods Course Topic: „High-Resolution Imaging”

05/2010 - 07/2010: Supervision of Research Project

Topic: „Live-cell imaging of quantum dots in neurons”

01/2009-03/2009: Supervision of Research Project

Topic: „The synaptic vesicle cycle: evidence for endosomal involvement"

\section{MisCELlanEOUS}

$07 / 2002-03 / 2003:$

Alternative Service ('Zivildienst') with ASB, Bremen

Individual care of a severely disabled patient

Languages:

German (native), English (fluent), French (basics)

IT skills:

Advanced knowledge of Microsoft Office and Adobe Creative Suite Advanced knowledge of programming in Matlab; basics in VBA 


\section{Publications}

Hoopmann P., Punge A., Barysch S.V., Westphal V., Bückers J., Opazo F., Bethani I.O., Lauterbach M.A., Hell S.W. and Rizzoli S.O. Endosomal sorting of readily releasable synaptic vesicles. in press

Hoopmann P., Rizzoli S.O. and Betz W.J. Imaging synaptic vesicle dynamics with FM dyes. Optical Imaging in Neuroscience (Book chapter). in press

Opazo F., Punge A., Bückers J., Hoopmann P., Kastrup L., Hell S.W., Rizzoli S.O. (2010). Limited Intermixing of Synaptic Vesicle Components upon Vesicle Recycling. Traffic 11(6): 800-812.

Geumann U., Barysch S.V., Hoopmann P., Jahn R. and Rizzoli S.O. (2008). SNARE function is not involved in early endosome docking. Mol Biol Cell 19(12): 5327-5337.

\section{Posters ANd Communications}

Hoopmann P., Punge A., Hell S.W. and Rizzoli S.O. (2009). Evidence for the Endosomal Recycling of Synaptic Vesicle Membrane. Poster: 2nd European Synapse Meeting, Göttingen.

Hoopmann P., Punge A. and Rizzoli S.O. (2009). Evidence for the Endosomal Recycling of Synaptic Vesicle Membrane. Poster: Society for Neuroscience Annual Meeting, Chicago, Ill., USA.

Hoopmann P. (2009). Superresolution Investigation of Synaptic Vesicle Recycling. Invited talk: Leica for Science Workshop, Munich.

Hoopmann P., Punge A. and Rizzoli S.O. (2008). The Molecular Composition of Synaptic Vesicles Changes during Exo-Endocytic Cycling. Poster: 5th International PhD Student Symposium Horizons in Molecular Biology, Göttingen. 